\title{
Precision measurements of the proton and deuteron polarized structure functions
}

Paul McConihay King II

College of William \& Mary - Arts \& Sciences

Follow this and additional works at: https://scholarworks.wm.edu/etd

Part of the Physics Commons

\section{Recommended Citation}

King, Paul McConihay II, "Precision measurements of the proton and deuteron polarized structure functions" (2000). Dissertations, Theses, and Masters Projects. Paper 1539623986.

https://dx.doi.org/doi:10.21220/s2-gay5-cj06

This Dissertation is brought to you for free and open access by the Theses, Dissertations, \& Master Projects at W\&M ScholarWorks. It has been accepted for inclusion in Dissertations, Theses, and Masters Projects by an authorized administrator of W\&M ScholarWorks. For more information, please contact scholarworks@wm.edu. 


\section{INFORMATION TO USERS}

This manuscript has been reproduced from the microfilm master. UMI films the text directly from the original or copy submitted. Thus, some thesis and dissertation copies are in typewniter face, while others may be from any type of computer printer.

The quality of this reproduction is dependent upon the quality of the copy submitted. Broken or indistinct print, colored or poor quality illustrations and photographs, print bleedthrough, substandard margins, and improper alignment can adversely affect reproduction.

In the unlikely event that the author did not send UMI a complete manuscript and there are missing pages, these will be noted. Also, if unauthorized copyright material had to be removed, a note will indicate the deletion.

Oversize materials (e.g., maps, drawings, charts) are reproduced by sectioning the original, beginning at the upper left-hand comer and continuing from left to right in equal sections with small overlaps.

Photographs included in the original manuscript have been reproduced xerographically in this copy. Higher quality $6^{*} \times 9^{*}$ black and white photographic prints are available for any photographs or illustrations appearing in this copy for an additional charge. Contact UMI directly to order.

Bell \& Howell Information and Leaming 300 North Zeeb Road, Ann Arbor, MI 48106-1346 USA 800-521-0600

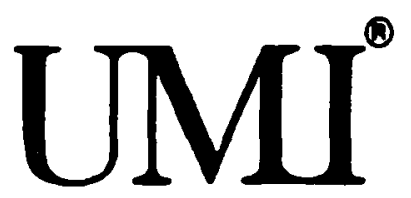




\section{PRECISION MEASUREMENTS OF THE PROTON AND DEUTERON POLARIZED STRLCTLRE FUNCTIONS}

A Dissertation
Presented to
The Faculty of the Department of Physics
The College of William and Mary in Virginia
In Partial Fulfilment
Of the Requirements for the Degree of
Doctor of Philosophy

by

Paul M. Kïng II

2000 
UMI Microform 9989347

Copyright 2001 by Bell \& Howell Information and Leaming Company. All rights reserved. This microform edition is protected against unauthorized copying under Title 17, United States Code.

Bell \& Howell Information and Learning Company 300 North Zeeb Road

P.O. Box 1346

Ann Arbor, MI 48106-1346 


\section{APPROVAL SHEET}

This dissertation is submitted in partial fulfillment of the requirements for the degree of

Doctor of Philosophy

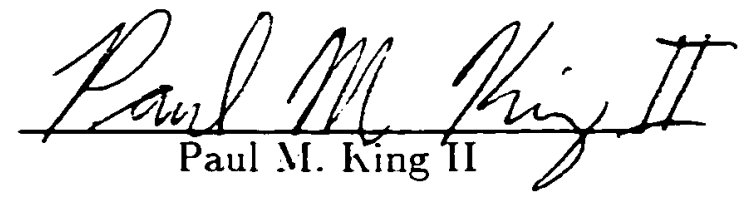

Approved. July 2000
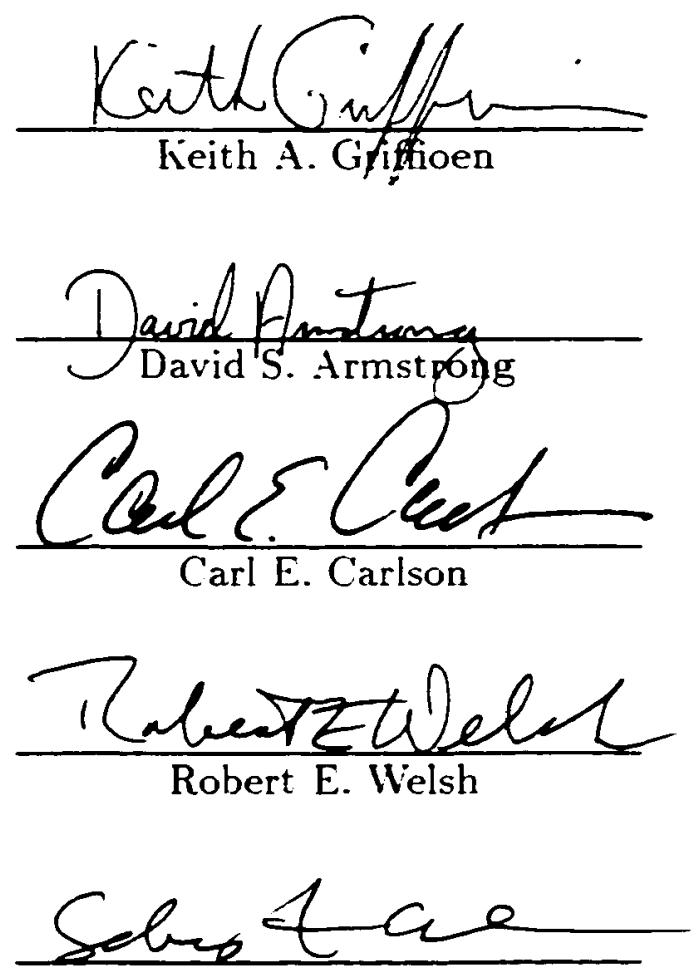

Sebastian E. Kuhn

Old Dominion University 
To my parents.

my family.

and other old friends. 


\section{CONTENTS}

ACKNOWLEDGMENTS .................... ix

LIST OF TABLES ..................... xi

LIST OF ILLUSTRATIONS .................. xv

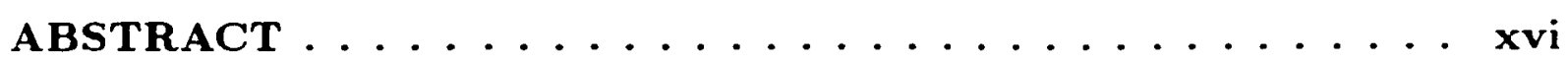

\section{CHAPTER}

1 INTRODUCTION AND THEORY .............. 2

1.1 Kinematics of Electron Scattering . . . . . . . . . . . 2

1.2 Deep Inelastic Lepton Scattering . . . . . . . . . . . . . 4

1.2.1 Enpolarized DIS ............. 6

1.2.2 Interpretation of the Unpolarized Structure Functions . . . $\$$

1.3 Polarized Deep Inelastic Lepton Scattering . . . . . . . . . 10

1.4 Spin Structure Function Evolution . . . . . . . . . . 15

1.5 Previous Polarized DIS Experiments . . . . . . . . . . 1 .

1.6 Operator Product Expansion . . . . . . . . . . . . . . . 19

1.7 Sum Rules ..................... 20

2 E155 EXPERIMENTAL EQUIPMENT . . . . . . . . . 22

2.1 The SLAC Polarized Electron Beam . . . . . . . . . . 23

2.2 Beam Transport ................... 28

2.2.1 The ESA Beam-line Elements . . . . . . . . . . 30

2.2.2 Beam Polarization Measurement . . . . . . . . . . 34 
2.2.3 ESA Chicane Magnet System ............ 40

2.3 Polarized Nuclear Target ................ 41

2.3.1 Target Assembly ................. 42

2.3 .2 Target Insert ...................... 43

2.3.3 Dynamic Nuclear Polarization ........... 4.5

2.3.4 Measurement of the Target Polarization ......... 46

2.4 Particle Detection and Identification: Spectrometers . . . . . . 49

2.4.1 Magnetic Optics \& Collimation ........... 51

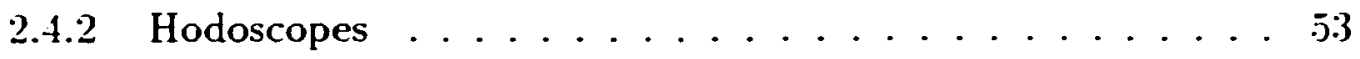

2.4.3 Threshold Cherenkov Detectors .......... 54

2.4.4 Electromagnetic Shower Calorimeter . . . . . . . . 59

2.5 Data Acquisition and Run Control ............ 63

2.5.1 Data Stream - Event and Non-event Information . . . . 64

3 THE $10.5^{\circ}$ SPECTROMETER ............... 66

3.1 Spectrometer Coordinate System ............. . 67

3.2 Magnetic Optics and Collimation ............... 67

3.3 Hodoscopes ...................... 72

3.4 Threshold Cherenkov Detector .............. it

3.5 Lead Glass Electromagnetic Shower Calorimeter . . . . . . . ij

3.5.1 Total Absorber ................... is

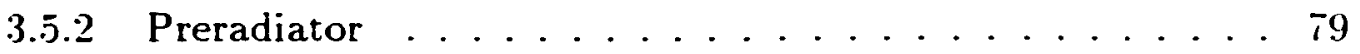

3.5.3 Installation Details ................ 81

3.5.4 Calorimeter Calibration and Checkout Before E1.5.5 ... 8.5

4 DATA COLLECTION: E155 \& T418 . . . . . . . . 87

4.1 E15.5: Longitudinal \& Transverse Asymmetry Measurement . . . . 88

4.1.1 E15.5 Longitudinal Asymmetry ........... 88 
4.1.2 E155 Transverse Asymmetry . . . . . . . . . . . 89

4.1 .3 Positron Runs ................... 89

4.1.4 Solid and Hole Target Runs ............ 89

4.1.5 Target Anneals \& Polarization ............. 90

4.1.6 Calibration Runs: Pedestal, LED. Toroid . . . . . . . . 91

4.1.7 Møller Runs ..................... 91

4.2 T418: Calibration Test Beam ............. 92

4.2.1 Beam Production and Delivery .......... 92

4.2.2 Mapping the Spectrometer Acceptances . . . . . . . . . . 94

4.2.3 10.5 Spectrometer Goals For T418 . . . . . . . . $99^{\top}$

5 DATA ANALYSIS .......................... 99

5.1 Raw Data Analysis .................... 100

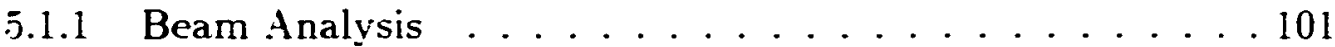

5.1.2 Spectrometer Data Unpacking and Calibration ...... 101

$5.1 .3 \quad$ Subsystem Analysis . . . . . . . . . . . . 103

5.2 Analysis of the $10.5^{\circ}$ Raw Data ........... 106

5.2.1 Modifications to the Tracking Code for the $10.5^{\circ}$ Analysis . 107

$5.2 .2 \quad 10.5^{\circ}$ Shower Calorimeter . . . . . . . . . . . 10

$5.2 .3 \quad 10.5^{\circ}$ Spectrometer Calibration Projects $\ldots . . . . .109$

5.3 Software Corrections in the $10.5^{\circ}$ Spectrometer Analysis . . . . . 115

5.3.1 Photomultiplier Tube Gain Variations due to Magnetic

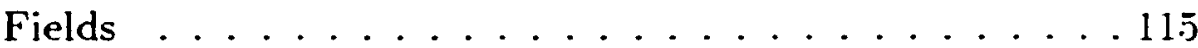

5.3.2 Calorimeter Pulse Width Behavior ......... . . . .

5.3.3 Preradiator Discriminator Thresholds . . . . . . . . 121

5.4 Data Summary Tape Production . . . . . . . . . . . 124

5.5 DST Re-Analysis: Creation of the Summary Files . . . . . . 125 
5.5.1 Event Selection Analysis from DST-2 ......... 127

5.6 Producing the DIS Asymmetries, $A_{\|} \& A_{\perp} \ldots \ldots . . \ldots .3 .3$

5.6.1 Raw Asymmetry Production: Asymmetries for Each Run . 134

5.6.2 Corrections for Contaminant Events . . . . . . . . . 144

5.6.3 Resolution Corrections .................. 147

5.6.4 Radiative Corrections . . . . . . . . . . . 148

5.7 Fully Corrected Deep Inelastic Scattering Asymmetries. $A_{\|} \& A_{\perp} \quad 149$

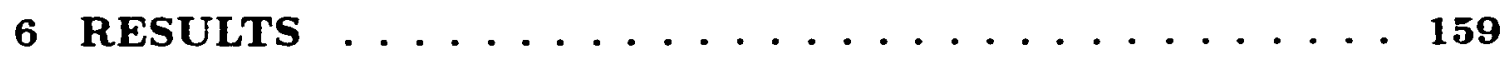

6.1 Virtual Photon Asymmetries. $A_{1} \& A_{2} \ldots \ldots . \ldots . . . . . . .59$

6.2 Spin Structure Functions, $g_{1} \& g_{2} \ldots \ldots 161$

6.3 Extraction of Neutron Asymmetries and Structure Functions . . . 161

7 INTERPRETATIONS ................. 167

7.1 Evolving $g_{1}$ to a common $Q^{2} \ldots \ldots . \ldots 16 \pi$

7.2 Sum rules involving $g_{2} \ldots \ldots$. . . . . . . . . . 169

7.3 The Operator Product Expansion Matrix Element. $d_{2} \ldots \ldots 9$

$7.4 Q^{2}$ Dependence and The Role of the $10.5^{\circ}$ Spectrometer . . . . 171

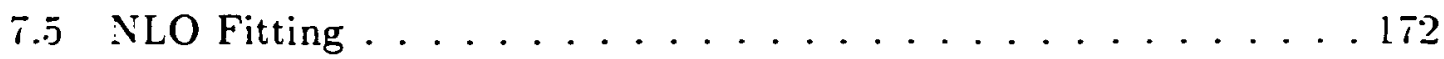

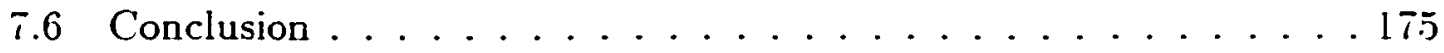

\section{APPENDIX A}

E155X: Measuring $A_{\perp} \ldots \ldots \ldots \ldots$

A.1 Lead Passive Preradiator (PbPPR) . . . . . . . . . 176

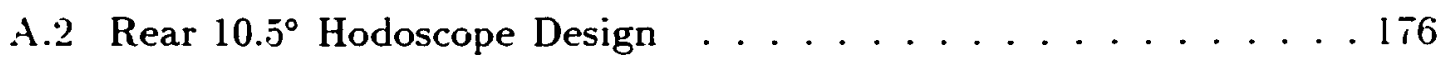

A.2.1 Construction ...........................

A.2.2 Assembly Notes . . . . . . . . . . . 179

A.2.3 Rough Positions of the Rear Detector Package Components 180

A.2.4 Finger Assembly Diagrams ............ 181

A.2.5 Photomultiplier Tube \& Base Assembly Diagrams . . . . . 182

vii 
A.3 Repairs to the Total Absorber Stack . . . . . . . . . 18.2

A.4 Effects of the $10.5^{\circ}$ Calorimeter $Z{ }^{-S h i f t}{ }^{-} \ldots \ldots$. . . . . 183

A.4.1 Effects on the time-of-flight correction . . . . . . . 184

A.4.2 Effects on the calculation of the trajectory slope . . . . 184

A.4.3 Observed effect of the $Z$ shift in E15.5X ........ 18.5

\section{APPENDIX B}

Bjorken $x$ in the infinite momentum frame ..........187

BIBLIOGRAPHY $\ldots \ldots \ldots \ldots$

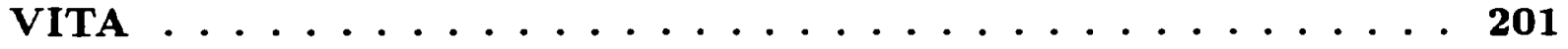

viii 


\section{ACKNOWLEDGMENTS}

I have been privileged to have seen the E1.5.5 $10.5^{\circ}$ spectrometer from its original construction. through the analysis of the data. to the upgrade and use in E1.5.5. I would like to thank my advisor. Keith Griffioen. for his help during this entire process, and especially during the last several months as I drew everything together.

I owe a great deal of thanks to the E1.55 collaboration and the SLAC staff. Special thanks go to Todd Averett. Peter Bosted. Carl Hudspeth. David Reyna. and Lee Sorrell. I also thank my fellow graduate students. Greg Mitchell. Terry Toole. Al Tobias. Paul McKee. and Frank Wesselmann. as well as the other students with whom I have worked, from William and Mary and from other institutions.

I would like to thank the members of my committee for their careful reading of this dissertation. I also thank my other readers. who assisted greatly in improving the early drafts of this document.

Sylvia Stout, Paula Perry, and Dianne Fannin have been essential during my time at William and Mary. I would like to recognize my friends in Williamsburg and elsewhere; it is invaluable to have friends who maintain their support during periods of frustration, despair, and extreme crankiness. Thank you all.

This work has been supported by Department of Energy grant number DEFG02-96ER41003. As a part of the Stanford Linear Accelerator Center research program. this work has been supported by Department of Energy contract DEAC03-76SF00515. 


\section{LIST OF TABLES}

1.1 GRV98 unpolarized parton distributions at $\mu^{2}=0.40\left(\mathrm{GeV}^{2} / c\right)^{2} \ldots \ldots 10$

1.2 Kinematic terms used in the relationships between $A_{\| 1} \cdot A_{\perp} \cdot A_{1}$ and $A_{2} .13$

1.3 A summary of polarized DIS experiments before E1.5. . . . . . . 18

$3.1 X$ and $Y$ survey data for the 9 May 1997 survey of the shower calorimeter. ....................... 69

3.2 Collimation periods for the $10.5^{\circ}$ spectrometer. . . . . . . i 1

3.3 Locations of the $10.5^{\circ}$ hodoscope fingers within the spectrometer co-

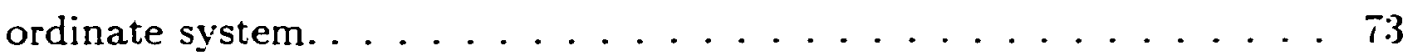

3.4 Locations of the $10.5^{\circ}$ shower calorimeter blocks within the spectrometer coordinate system. . . . . . . . . . . . . . \$:3

4.1 Number of runs used in the physics analysis for each spectrometer for different spectrometer polarities, target orientations. and target materials. ................... 88

4.2 Grid patterns used for each set of beam energy and spectrometer magnet settings during T418. . . . . . . . . . 95

5.1 Spectrometer subsystems included in each track type. . . . . . 10.5

5.2 First pass $10.5^{\circ}$ calorimeter TDC slewing corrections . . . . . . 113

5.3 Final $10.5^{\circ}$ calorimeter TDC slewing corrections . . . . . . . . 113

5.4 Standard electron and pion definitions for the $2.75^{\circ}$ and $5.5^{\circ}$ analyses. 126 
5.5 The three subdefinitions of the student implementation of the $10.5^{\circ}$

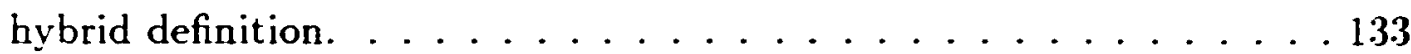

5.6 World data set used in the global fit to $A_{1} \ldots \ldots \ldots$

5.7 Final $A_{\|}^{p}$ and $A_{\|}^{d}$ for the $2.75^{\circ}$ spectrometer. . . . . . . . . . 2

5.8 Final $A_{\|}^{p}$ and $A_{\|}^{d}$ for the $5.5^{\circ}$ spectrometer. . . . . . . . . . . .

5.9 Final $A_{\|}^{p}$ and $A_{\|}^{d}$ for the $10.5^{\circ}$ spectrometer. . . . . . . . . . .

5.10 Final $A_{\perp}^{p}$ and $A_{\perp}^{d}$ for the $2.75^{\circ}$ spectrometer. . . . . . . . . . .

5.11 Final $A_{\perp}^{p}$ and $A_{\perp}^{d}$ for the $5.5^{\circ}$ spectrometer. ...........

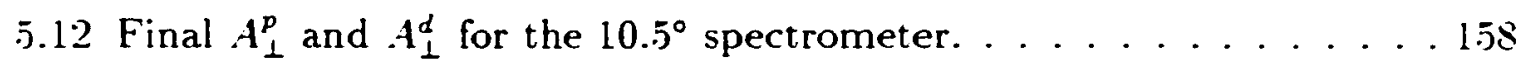

6.1 E15.5 Proton results for $g_{1} / F_{1}$ and $g_{1} \ldots \ldots \ldots \ldots 2$

6.2 E155 deuteron results for $g_{1} / F_{1}$ and $g_{1} \ldots \ldots \ldots . \ldots \ldots$

$6.3 \quad$ E $15.5 A_{2}$ and $x g_{2}$ for the proton and deuteron. . . . . . . . . 164

i.l The parameters of the global fit to $A_{1}$ with their errors for three sets of world data: excluding E15.5. including the E1.5.52.7.5 and $5.5^{\circ}$ spectrometers. and including all of the E155 data. . . . . . . 172

7.2 Coefficients of the NLO pQCD fit of the polarized parton distributions.174

A.1 Estimated positions of the elements of the E155X $10.5^{\circ}$ rear detector

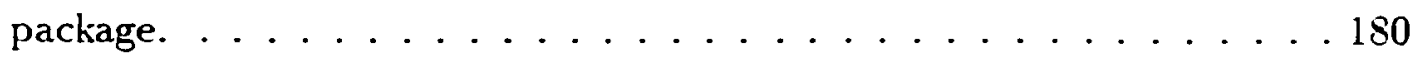




\section{LIST OF FIGURES}

1.1 Lowest order diagram for deep inelastic lepton scattering. . . . . . . 3

1.2 Deep inelastic electron scattering Feynman diagram. . . . . . . . . 4

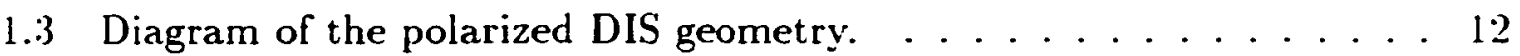

2.1 Block diagram of a polarized DIS experiment. . . . . . . . 22

2.2 Drawing of the SLAC polarized electron source. . . . . . . 2.5

2.3 Schematic of the algorithm used to determine the polarization bit from the 32 bit register. . . . . . . . . . . 25

2.4 Energy level diagram of bulk Ga.As and strained Cia.ts. . . . . . 26

2.5 Drawing of the A-Line. . . . . . . . . . 28

2.6 "Spill maps" of average bad spill signal and good spill signal across the raster range for Run $3450 \ldots \ldots \ldots \ldots \ldots . \ldots \ldots 2$

2.7 The E154 Møller polarimeter detector package. . . . . . . . . 38

2.8 Rough schematic of the chicane system. . . . . . . . 40

2.9 Schematic cross-section of the target assembly. . . . . . . 42

2.10 Energy level schematic for the electron-proton system in an external magnetic field. . . . . . . . . . . . . . . 4.5

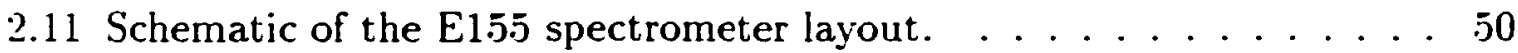

2.12 E15.5 kinematic coverage per spectrometer at a beam energy of 48.3

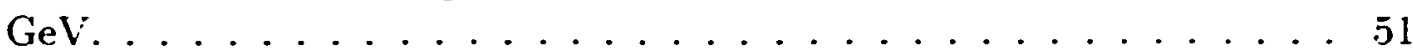

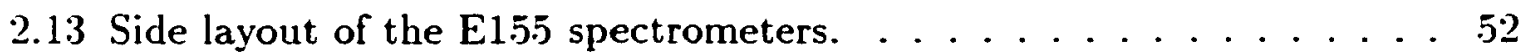
xii 
2.14 Plot of the Cherenkov light production as a function of the particle momentum in units of $p_{\min }$ at fixed values of $\omega^{2}$ and $n . \ldots . .56$

2.15 Average pedestal-subtracted Cherenkov waveform. . . . . . . . 59

2.16 Diagram of the $2.75^{\circ}$ calorimeter blocks seen from the rear. . . . . 63

3.1 View of the bend plane of the $10.5^{\circ}$ spectrometer optics model. . . . 68

3.2 View of the non-bend plane of the $10.5^{\circ}$ spectrometer optics model. . 68

3.3 Side view diagram of the $10.5^{\circ}$ hodoscope straight and bent fingers. - i2

3.4 Cherenkov average waveform and spectrum of $V_{\text {peak }}$ for Run 3340. . . 76

3.5 Drawing of the $10.5^{\circ}$ shower calorimeter. showing the TA blocks and PR bars. ...................... Ti

3.6 Diagram of the TA block faces. looking downstream. ...... is

3.7 Side view of the $10.5^{\circ}$ shower calorimeter assembly and stand. . . . . \$2

3.8 Diagram of the TA and PR stacks with lead shield walls. looking from

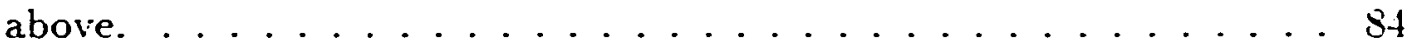

4.1 Diagram of steering magnet and beam hodoscope locations in the BSY $Z$ reference system. . . . . . . . . . . . . . . 93

5.1 The raw $10.5^{\circ}$ Cherenkov FADC waveform in a spill without a Cherenkov hit. ....................... 103

5.2 Average pedestal-subtracted Cherenkov waveform. . . . . . . . 104

5.3 Diagram showing the hardware pedestal with three pulses. . . . . 110

5.4 Diagram showing time slewing difference between two pulses starting at the same time but having different amplitudes. . . . . . . . 112

5.5 Diagram showing the effects of an insufficiently long ADC gate upon the amount of charge integrated by two pulses at different times. . . . 114

xiii 
5.6 Average of the fractional difference between each TA LED response and the LED response for that TA block during Run 1902. . . . . 116

5.7 Sample FADC waveform of TA block 3.4 showing ringing on the trailing edge. . . . . . . . . . . . . . . . .

5.8 Contour plot of charge versus time difference between adjacent TDC hits. . . . . . . . . . . . . . . . 120

5.9 Beam current dependence of the preradiator energy spectra. . . . . 122

5.10 Preradiator energy spectra with and without TDC events. . . . . 12:3

5.11 Four spectra used in event definition in the $10.5^{\circ}$ spectrometer. . . . 128

5.12 Comparison of $A_{\|}^{p}$ using either the recombination or reclustering shower calorimeter analyses. . . . . . . . . . . I30

5.13 Comparison of $A_{\|}^{p}$ using either the track-based or shower-based analyses. I31

5.14 Comparison of $A_{\|}^{p}$ using either the track momentum or shower energy

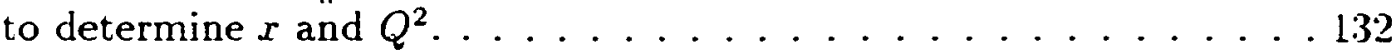

5.15 Average dilution factor in the $10.5^{\circ}$ spectrometer in each $x$-bin for the proton and deuteron target materials. . . . . . . . 13i

5.16 Average multiplicative nuclear correction term. $C_{1}$, in each $x$-bin for the proton and deuteron target materials. . . . . . . . 138

5.17 Comparison of asymmetry with and without EW correction. . . . . 142

5.18 The difference between the proton asymmetry with and without the EW correction, quantity divided by the uncorrected asymmetry. . . 14:3

5.19 The ratio of positron rate to electron rate in the $10.5^{\circ}$ spectrometer for two definitions. . . . . . . . . . . . . . 14t

5.20 Comparison of asymmetry with and without pion/positron correction. $14 i$

5.21 Comparison of asymmetry with and without radiative corrections. . . 150

5.22 Fully corrected $A_{\|\|}^{p}$ and $A_{\|}^{d}$ for the three spectrometers at the measured

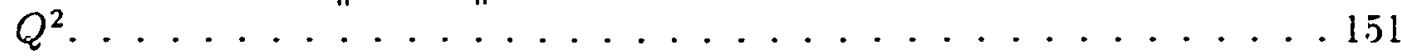

5.23 Fully corrected $A_{\perp}^{p}$ and $A_{\perp}^{d}$ for the three spectrometers at the measured $Q^{2}$.

xiv 
6.1 Plot of E1.55 $A_{2}$ for the proton and deuteron vs $Q^{2}$ for selected bins in $x \ldots \ldots \ldots \ldots \ldots \ldots$

6.2 E15.5 $g_{2}$ results averaged over spectrometers and data from E143. . . 164

6.3 Combined $g_{1}$ from all three spectrometers evolved to a $Q^{2}$ of $5(\mathrm{GeV} / \mathrm{c})^{2}$. for the proton, deuteron, and extracted neutron. . . . . . . . . 166

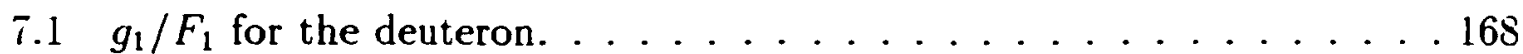

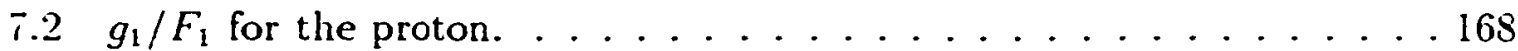

7.3 The combined SL.AC determination of the leading twist-3 matrix element $d_{2}$ with model predictions. . . . . . . . . . . 170

A.1 E155X rear hodoscope lightguide diagrams. . . . . . . . . I81

A.2 E15.5X rear hodoscope photomultiplier tube assembly diagrams. . . . 1\$2

A.3 Plots of trajectory slope. $d Y / d Z$. and $E / P$ for electron tracks in a

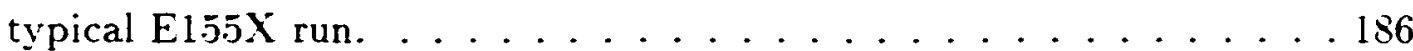




\begin{abstract}
Experiment E1.5.5 at the Stanford Linear Accelerator Center measured the longitudinal and transverse polarized deep-inelastic scattering asymmetries for the proton and deuteron using longitudinally-polarized electron beams with energies of 48.3 $\mathrm{GeV}$ and $38.8 \mathrm{GeV}$, respectively. The electron beam polarization was measured by Moller polarimetry to be $0.81 \pm 0.02$. Dynamically-polarized solid ${ }^{15} \mathrm{NH}_{3}$ and ${ }^{6} \mathrm{Li}^{2} \mathrm{H}$ were used as the target materials. The scattered electrons were detected in three independent spectrometers at $2.75^{\circ}, 5.5^{\circ}$. and $10.5^{\circ}$. covering a kinematic range of $0.014 \leq x \leq 0.9$ and $1.0(\mathrm{GeV} / c)^{2} \leq Q^{2} \leq 40(\mathrm{GeV} / c)^{2}$. The $10.5^{\circ}$ spectrometer was constructed for this experiment. The structure functions $g_{1}^{p}$ and $g_{1}^{d}$ were extracted from the asymmetry data, and, in combination with world data. were fit in nextto-leading order perturbative QCD. The $Q^{2}$ dependence of $g_{1}$ differed slightly from that of $F_{1}$. The $g_{1}$ results are consistent with the Bjorken sum rule. The structure functions $g_{2}^{p}$ and $g_{2}^{d}$ were extracted from the asymmetry data. and were used to extract the twist -3 reduced matrix elements $d_{2}^{p}, d_{2}^{d}$. and $d_{2}^{n}$.
\end{abstract}




\section{PRECISION MEASUREMENTS OF THE PROTON AND DELTERON POLARIZED STRUCTURE FUNCTIONS}




\section{CHAPTER 1 \\ INTRODUCTION AND THEORY}

The constituent quark model, in which the nucleon is composed of the three valence quarks. each of which has a mass of about $1 / 3$ the nucleon mass. works fairly well to describe the static properties and the baryonic mass spectrum. But we know that at higher energies the constituent quark picture does not work. and the sea quarks and gluons play a large part in determining the nucleon's properties. The interactions between the quarks and gluons become quite significant in describing the behavior of the nucleon at these scales. At still higher energy scales. the quarks and gluons do not interact with each other, due to the asymptotic freedom of quantum chromodynamics (QCD). The question of how the high-energy asymptotic freedom of QCD relates to the low-energy behavior of the hadrons is still unanswered. Polarized and unpolarized deep inelastic scattering experiments address this question by probing the nucleon structure over a wide range of momentum transfers.

Experiment E15.5. which was conducted at the Stanford Linear Accelerator Center (SLAC). used polarized deep inelastic scattering to measure the spin structure of the proton and deuteron over a range in the square of the 4-momentum transfer. $Q^{2}$. of $1(\mathrm{GeV} / c)^{2}$ to $40(\mathrm{GeV} / c)^{2}$.

\subsection{Kinematics of Electron Scattering}

The lowest order process by which neutral-current lepton scattering can take place is shown in Fig. 1.1. The incident lepton. with 4-momentum $k^{\mu}=(E . \vec{k})$. scatters from the nucleon, with 4-momentum $P^{\mu}=(M . \overrightarrow{0})$. by exchanging a virtual photon, $\gamma^{-}$, or $Z^{0}$ with 4-momentum $q^{\mu}=k^{\mu}-k^{\prime \mu}$. The 4-momentum of the 


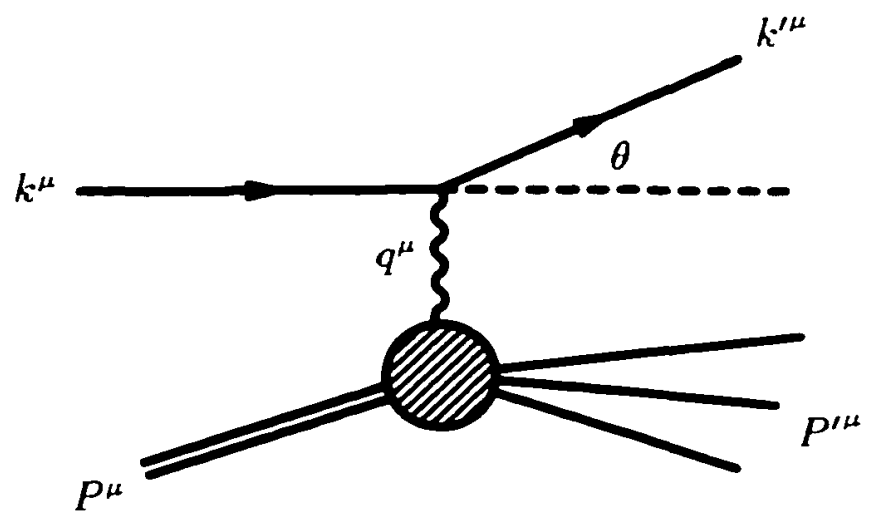

Figure 1.1: Lowest order diagram for deep inelastic lepton scattering. The incident and outgoing electron 4-momenta are respectively: $k^{\mu}=(E \cdot \vec{k})$ and $k^{\prime \mu}=\left(E^{\prime} \cdot \overrightarrow{k^{\prime}}\right)$. The nucleon initially has 4-momentum $P^{\mu}$ : after the collision. the outgoing hadronic system has 4-momentum $P^{\prime \mu}$.

outgoing lepton is $k^{\prime \mu}=\left(E^{\prime}, \overrightarrow{k^{\prime}}\right)$. and the outgoing hadronic system has 4-momentum $P^{\mu}=P^{\mu}+q^{\mu}$. The invariant quantities which describe the interaction are

$$
\begin{aligned}
Q^{2} \equiv-q^{2} & =-\left(k^{\mu}-k^{\mu}\right)^{2} \\
\nu & =P_{\mu} q^{\mu} / M \\
W^{-2} & =\left(P^{\prime}\right)^{2}=M^{2}-Q^{2}+2 P_{\mu} q^{\mu} \\
x & =\frac{-q^{2}}{2 P_{\mu} q^{\mu}} \\
s & =\left(P^{\mu}+k^{\mu}\right)^{2}
\end{aligned}
$$

where.$/$ is the nucleon mass, and the lepton mass has been neglected. The quantity $\nu$ is the energy of the virtual photon as evaluated in the nucleon rest frame. $W^{-2}$ is the square of the invariant mass of the outgoing hadronic system. $s$ is the square of the center-of-mass energy of the collision. and the Bjorken scaling variable. $x$, will be discussed in more detail below and in Appendix B. In the laboratory frame. with the nucleon initially at rest. the kinematic variables are

$$
\begin{aligned}
Q^{2} & =4 E E^{\prime} \sin ^{2} \frac{\theta}{2} \\
\nu & =E-E^{\prime} \\
W^{-2} & =M^{2}-Q^{2}+2 M \nu \\
x & =Q^{2} /(2 M \nu)
\end{aligned}
$$




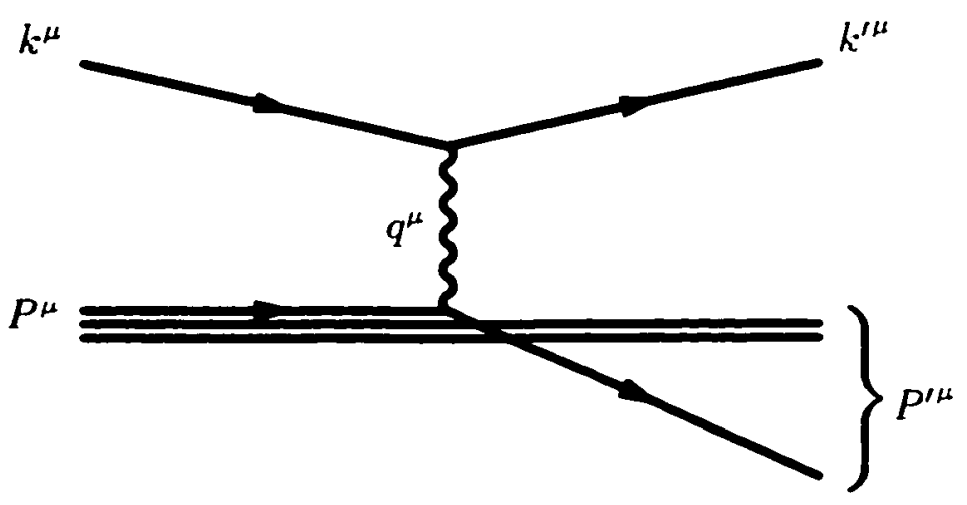

Figure 1.2: Deep inelastic electron scattering Feynman diagram.

where $E$ is the incident lepton energy. and $E^{\prime}$ and $\theta$ are the energy and scattering angle of the outgoing lepton.

The resolving power of the virtual photon (photon wavelength) is $\hbar / \sqrt{Q^{2}}$ [1]. so as $Q^{2}$ increases, the distance scale probed by the interaction decreases. For lowenergy lepton-nucleon collisions, elastic scattering dominates with $U^{-2}=M^{2}$ and $\nu=Q^{2} / 2 M$.

As the interaction energy increases. with $Q^{2}$ increasing above $0.5(\mathrm{GeV} / \mathrm{c})^{2}$. the form factors of the nucleon and the resonances become less significant and the interaction begins to probe the substructure of the nucleon. It is in this high-energy scattering, where $Q^{2}$ and $\nu$ are large, that deep inelastic scattering can be used to study the quark distributions in the nucleon.

\subsection{Deep Inelastic Lepton Scattering}

In deep inelastic lepton scattering. $Q^{2}$ and $\nu$ are both large. but the scaling variable $x$ is finite. The virtual photon has a small wavelength and interacts with one of the partons within the nucleon, as is shown in Fig. 1.2. This scaling variable. $x$, is called Bjorken $x$ and, in the infinite moment um frame. represents the fraction of the proton momentum carried by the struck parton (see Appendix B). In the infinite momentum frame, the transverse momentum of the partons within the proton may be neglected. 
The partons are either quarks and gluons; however. only the quarks contribute to deep inelastic scattering at the lowest order in QCD. since the gluons carry no weak or electric charge. In deep inelastic scattering, the cross-sections measured are dependent upon the momentum distributions of the quarks and anti-quarks.

The general deep inelastic scattering (DIS) cross-section is proportional to the product of the leptonic and hadronic tensors. $L_{\mu \nu}$ and $W_{\mu \nu}$.

$$
\left.\sigma \sim L_{\mu \nu} W^{\mu \nu}=L_{\mu \nu}(\text { sym. }) W^{\mu \nu}(\text { sym. })+L_{\mu \nu}(\text { antisym. }) W^{-\mu \nu} \text { (antisym. }\right)
$$

where $L_{\mu \nu}($ sym. $)$ and $W^{\mu \nu}$ (sym.) are the part of the leptonic and hadronic tensors symmetric under exchange of $\mu$ and $\nu$. and $L_{\mu \nu}$ (antisym.) and $W^{\mu \nu}$ (antisym.) are antisymmetric [2]. The symmetric tensors are independent of the lepton and hadron polarization vectors, but the antisymmetric tensors are linearly dependent upon the respective polarization vectors,

$$
\begin{gathered}
L_{\mu \nu}(\text { antisym. })=2 i m \epsilon_{\mu \nu \alpha 3} q^{\alpha} s^{3} . \\
W_{\mu \nu}(\text { antisym. })=i \frac{M}{P q} \epsilon_{\mu \nu \rho \sigma} q^{\rho}\left[S^{\sigma} g_{1}\left(x . Q^{2}\right)+\left(S^{\sigma}-\frac{S q}{P q} P^{\sigma}\right) g_{2}\left(x, Q^{2}\right)\right] .
\end{gathered}
$$

where $m$ is the lepton mass, $\boldsymbol{M}$ is the nucleon mass. $s^{\mu}$ is the lepton polarization 4 vector. $q^{\mu}$ is the 4 -momentum transfer. $S^{\mu}$ is the nucleon polarization 4 -vector. $P^{\mu}$ is the nucleon 4-momentum, $\epsilon_{\mu \nu \rho \sigma}$ is the fully antisymmetric tensor in four dimensions. and $g_{1}$ and $g_{2}$ are the polarized structure functions [2]. Longitudinally-polarized electrons have the polarization vector $s^{\mu}= \pm \frac{1}{m}\left(\sqrt{E^{2}-m^{2}} \cdot 0.0 . E\right)[2]$. where the positive sign corresponds to right-handed electrons (spin parallel to momentum) and the negative sign corresponds to left-handed electrons (spin anti-parallel to momentum); notice that for high energies. $m s^{\mu}$ converges to $\pm p^{\mu}[2]$. The nucleon polarization 4 -vector is

$$
S^{\mu}=\left(\frac{\vec{P} \cdot \vec{\xi}}{M}, \vec{\xi}+\frac{(\vec{P} \cdot \vec{\xi}) \vec{P}}{M(E+M)}\right)
$$


where $\vec{\xi}$ is the unit vector along the direction of polarization in the nucleon rest frame [1]. By reversing one of the polarization vectors, the symmetric product does not change, but the antisymmetric product changes sign.

\subsubsection{Unpolarized DIS}

In an unpolarized DIS measurement, the spin-dependent part of the crosssection is averaged out, yielding only the portion of the cross-section which is proportional to $L_{\mu \nu}(\operatorname{sym}.) W^{-\mu \nu}$ (sym.). The unpolarized deep inelastic electron scattering cross-section is

$$
\frac{d^{2} \sigma_{\text {unpol. }}}{d \Omega d E^{\prime}}=\sigma_{\text {Mott }}\left[2 \tan ^{2} \frac{\theta}{2} W_{1}\left(\nu, Q^{2}\right)+W_{2}\left(\nu \cdot Q^{2}\right)\right]
$$

where the Mott cross-section. which describes electron scattering in a Coulomb field. is

$$
\sigma_{\mathrm{Mott}}=\frac{\alpha^{2}}{4 E^{2} \sin ^{4} \frac{\theta}{2}} \cos ^{2} \frac{\theta}{2}=\frac{4 E^{\prime 2} \alpha^{2}}{Q^{4}} \cos ^{2} \frac{\theta}{2} .
$$

$\alpha \approx 1 / 13 T$ is the fine-structure constant. and the structure functions $W_{1}$ and $W_{2}$ are functions of $\nu$ and $Q^{2}[1] . W_{1}$ and $W_{2}$ can be related to the absorption cross-sections for virtual transverse and longitudinal photons. $\sigma_{T}$ and $\sigma_{L}$.

$$
\begin{aligned}
& W_{1}\left(\nu, Q^{2}\right)=\frac{\sqrt{\nu^{2}+Q^{2}}}{4 \pi \alpha^{2}} \sigma_{L} \\
& W_{2}\left(\nu, Q^{2}\right)=\frac{Q^{2}}{4 \pi \alpha^{2} \sqrt{\nu^{2}+Q^{2}}}\left(\sigma_{L}+\sigma_{T}\right)
\end{aligned}
$$

Eq. 1.16 and 1.17 imply that the ratio of $W_{1}$ and $W_{2}$ can be written in terms of the ratio of the absorption cross-sections for virtual longitudinal and transverse photons, $R=\sigma_{L} / \sigma_{T}$, as follows $[1]$

$$
\frac{W_{1}\left(\nu, Q^{2}\right)}{W_{2}\left(\nu, Q^{2}\right)}=\left(1+\frac{\nu^{2}}{Q^{2}}\right)(1+R)^{-1} .
$$

In the Bjorken scaling limit, where $\nu, Q^{2} \rightarrow \infty$ with $x=Q^{2} /(2 M \nu)$ remaining finite, $M W_{1}$ and $\nu W_{2}$ depend only on $x$, and therefore we define two new structure 
functions,

$$
\begin{aligned}
M W_{1}\left(\nu \cdot Q^{2}\right) & \rightarrow F_{1}(x) \\
\nu W_{2}\left(\nu \cdot Q^{2}\right) & \rightarrow F_{2}(x) .
\end{aligned}
$$

At finite values of $\nu, Q^{2}$. the scaling is not exact. and $F_{1}$ and $F_{2}$ are functions of both $x$ and $Q^{2}$. The cross-section, expressed using $F_{1}$ and $F_{2}$ is

$$
\frac{d^{2} \sigma_{\text {unpol. }}}{d \Omega d E^{\prime}}=\sigma_{\text {Mott }}\left[2 \tan ^{2} \frac{\theta}{2} F_{1}\left(x \cdot Q^{2}\right) / M+F_{2}\left(x \cdot Q^{2}\right) / \nu\right]
$$

Reformulating Eq. 1.18 in terms of $F_{1}$ and $F_{2}$ yields [3]

$$
\frac{F_{1}}{F_{2}}=\frac{M}{\nu}\left(1+\frac{\nu^{2}}{Q^{2}}\right)(1+R)^{-1} .
$$

and

$$
R\left(x, Q^{2}\right)=\frac{F_{2}\left(x, Q^{2}\right)}{2 x F_{1}\left(x, Q^{2}\right)}\left(1+\frac{4 M^{2} x^{2}}{Q^{2}}\right)-1
$$

A large amount of unpolarized DIS data. using both electron and muon beams. have been collected. A representative sample [4] of the experimental measurements of $F_{2}^{p}$ and $F_{2}^{d}$ includes electron DIS data from SLAC [5], H1 [6. 7]. and ZELS [8. 9]. and muon DIS data from NMC [10]. E66.5 [11], and BCDMS [12. 13]. The $Q^{2}$ range extends from about $0.1(\mathrm{GeV} / \mathrm{c})^{2}$ to about $5000(\mathrm{GeV} / \mathrm{c})^{2}$. with coverage in $x$ from about $10^{-6}$ through $0.85[4]$.

The NMC collaboration performed fits to $F_{2}^{p}$ and $F_{2}^{d}[10]$ using the combined NMC. SLAC [5], and BCDMS $[12,13]$ data. These fits. "F2NMC". use a fifteen parameter function for each of $F_{2}^{p}$ and $F_{2}^{d}$ to describe the structure functions over the kinematic range of $0.006<x<0.9$ and from about $0.5(\mathrm{GeV} / \mathrm{c})^{2}$ to $200(\mathrm{GeV} / \mathrm{c})^{2}$ in $Q^{2}$.

The structure function $R$ has also been measured by several experiments as noted in Ref. [14], such as: SLAC E140 [15], SLAC E140X [16]. SLAC E143 [14], NMC [17], CCFR, and CDHSW [18]. The E143 collaboration has parameterized $R$ 
[14]: this fit. "R1998". includes data with the kinematic range $0.005 \leq x \leq 0.86$ and $0.5 \leq Q^{2} \leq 130$. and is based upon the earlier SLAC parameterization. R1990 [1.5]. The R1998 parameterization combines data on a variety of nuclear targets. since $R^{p}=R^{d}=R^{A}$ to within the accuracy of available measurements [14].

\subsubsection{Interpretation of the Unpolarized Structure Functions}

The goal of the unpolarized structure function measurements is to quantify or constrain the contributions from the various types of partons. In the naive quarkparton model. the unpolarized structure functions are described in terms of the quark $x$ distributions,

$$
\begin{aligned}
& F_{1}(x)=\frac{1}{2} \sum_{i=u, d, s} e_{i}^{2} q_{i}(x) \\
& F_{2}(x)=\sum_{i=u, d . s} e_{i}^{2} x q_{i}(x)
\end{aligned}
$$

where $e_{i}$ is the electric charge of the quark flavor and $q_{i}(x)$ describes the number of quarks and anti-quarks of each flavor which have the momentum fraction. $x$ [1]. The sum over quark flavors is restricted to the three lightest flavors (u.d.s). Within this model the quark distributions and the structure functions are entirely independent of $Q^{2}$ : i.є., Bjorken scaling is exact. Since there are observed $Q^{2}$ dependencies. the naive quark-parton model must neglect aspects of the problem.

The naive quark-parton model treats the quarks as non-interacting and weakly bound, neglecting the effects of the gluons. The gluons give rise to a $Q^{2}$ dependence in the quark distributions through gluon radiation (quarks can radiate some of their 4-momentum), and can also contribute to the structure functions through photongluon fusion, or quark-gluon correlations. In perturbative QCD, the unpolarized structure functions are described by,

$$
F\left(x, Q^{2}\right)=\sum_{i}^{N_{f}} e_{i}^{2}\left[C_{q} \odot q_{i}+\frac{1}{N_{f}} C_{G} \odot G\right]
$$


where $N_{f}$ is the number of active quark flavors (usually restricted to the three lightest flavors), $C_{q}$ and $C_{G}$ are the Wilson coefficient functions and depend upon both $x$ and $\alpha_{S}, G$ is the gluon distribution function, and $\alpha_{S}$ is the coupling constant of the strong force. The convolution, $C \odot q$, is

$$
(C \odot q)\left(x, Q^{2}\right)=\int_{x}^{1} C\left(z \cdot \bar{g}^{2}\right) q\left(x / z, Q^{2}\right) \frac{d z}{z}
$$

where $q$ is taken to be either a quark or gluon parton distribution [1].

Instead of using the quark and anti-quark distributions as described above. other parton definitions are defined using the quark distributions $\left(u\left(x, Q^{2}\right), d\left(x, Q^{2}\right)\right.$. $\left.s\left(x, Q^{2}\right)\right)$ and antiquark distributions $\left(\bar{u}\left(x, Q^{2}\right), \bar{d}\left(x, Q^{2}\right), \bar{s}\left(x, Q^{2}\right)\right):$ these distributions are related to the distributions above by $q_{u}=u+\bar{u}, q_{d}=d+\bar{d}$. and $q_{s}=s+\bar{s}$. The $u$ ard $d$ quark and antiquark distributions are further separated into the valence contribution and sea contribution. The antiquark distribution describes the distribution of both quarks and antiquarks in the sea. $\left(u_{\text {sea }}\left(x, Q^{2}\right) \equiv \bar{u}\left(x, Q^{2}\right)\right)$, and the valence quark distributions are

$$
\begin{aligned}
& u_{v}\left(x, Q^{2}\right) \equiv u\left(x, Q^{2}\right)-u_{\text {sea }}\left(x, Q^{2}\right)=u\left(x, Q^{2}\right)-\bar{u}\left(x, Q^{2}\right) \\
& d_{v}\left(x, Q^{2}\right) \equiv d\left(x, Q^{2}\right)-d_{\text {sea }}\left(x, Q^{2}\right)=d\left(x, Q^{2}\right)-\bar{d}\left(x, Q^{2}\right) .
\end{aligned}
$$

Beginning at a low input scale $Q^{2}=\mu^{2}$. the following parton distributions are parameterized: the valence quarks, $u_{V}\left(x, \mu^{2}\right)$ and $d_{v}\left(x, \mu^{2}\right): \Delta\left(x, \mu^{2}\right) \equiv \bar{d}-$ $\bar{u}$. which describes the difference in $u$ and $d$ sea quarks: $(\bar{d}+\bar{u})\left(x \cdot \mu^{2}\right)$. the nonstrange sea quarks; $G\left(x, \mu^{2}\right)$. the gluons; and the strange sea. $s\left(x, \mu^{2}\right)$ and $\bar{s}\left(x \cdot \mu^{2}\right)$. These parton distribution parameterizations are evolved from the input scale to the experimental $Q^{2}$ using the Dokshitzer-Gribov-Lipatov-Altarelli-Parisi (DGLAP) equations $[19,20,21,22]$,

$$
Q^{2} \frac{d}{d Q^{2}} q_{N S}^{\eta}=\frac{\alpha_{s}\left(Q^{2}\right)}{2 \pi} P_{N S}^{\eta} \odot q_{N S}^{\eta}, \quad \eta= \pm 1
$$




$$
\begin{aligned}
x u_{V}\left(x, \mu^{2}\right) & =0.632 x^{0.43}(1-x)^{3.09}(1+18.2 x) \\
x d_{V}\left(x, \mu^{2}\right) & =0.394 x^{0.43}(1-x)^{4.09}(1+18.2 x) \\
x \Delta\left(x, \mu^{2}\right) & =0.20 x^{0.43}(1-x)^{12.4}(1-13.3 \sqrt{x}+60.0 x) \\
x(\bar{u}+\bar{d})\left(x, \mu^{2}\right) & =1.24 x^{0.20}(1-x)^{8.5}(1-2.3 \sqrt{x}+5.7 x) \\
x G\left(x, \mu^{2}\right) & =20.80 x^{1.6}(1-x)^{4.1} \\
x s\left(x, \mu^{2}\right)=x \bar{s}\left(x, \mu^{2}\right) & =0
\end{aligned}
$$

Table 1.1: GRV98 unpolarized parton distributions in NLO QCD at the input scale. $\mu^{2}=0.40(\mathrm{GeV} / \mathrm{c})^{2}$, from Ref. [24].

$$
Q^{2} \frac{d}{d Q^{2}}\left(\begin{array}{c}
\Sigma \\
G
\end{array}\right)=\frac{\alpha_{s}\left(Q^{2}\right)}{2 \pi}\left(\begin{array}{cc}
P_{q q} & P_{q G} \\
P_{G q} & P_{G G}
\end{array}\right) \odot\left(\begin{array}{c}
\Sigma \\
G
\end{array}\right) .
$$

where $q_{V S}^{+1}$ stands for the (non-singlet) valence distributions. $u_{v}$ and $d_{v} ; q_{V S}^{-1}$ stands for the $S\left[i(3)_{\text {flavor }}\right.$ non-singlet combinations $q_{3}=u-d$. and $q_{8}=u+d-2 s[23]$. The singlet distributions are the quark distribution $\Sigma\left(x . Q^{2}\right)=\sum_{i=u . d . s} q_{i}$ and the gluon distribution $G\left(x, Q^{2}\right)$. The splitting functions. $P\left(x, \alpha_{s}\right)$, are pert urbative expansions similar to $C\left(x, \alpha_{s}\right)$.

The evolved parameterizations are used in Eq. 1.26 to fit the unpolarized structure function data. and therefore determine the parameter values. The GRV 98 [24] unpolarized parton distributions, shown in Table 1.1, are evaluated in nextto-leading order QCD using the $\overline{\mathrm{MS}}$ scheme $[2.5,26]$. at an input scale of $\mu^{2}=$ $0.40(\mathrm{GeV} / \mathrm{c})^{2}$. The strange sea was chosen to vanish at the input scale: the $Q^{2}>\mu^{2}$ strange quark distributions are generated dynamically through the evolution; i.t.. they are radiatively generated [24].

\subsection{Polarized Deep Inelastic Lepton Scattering}

In polarized deep inelastic lepton scattering, both the incident lepton and the target nucleon are polarized ${ }^{1}$. so both the spin-independent and spin-dependent

\footnotetext{
${ }^{1}$ Recall that Eq. 1.10 does not have contributions from terms that depend on the lepton or nucleon polarization independently; within this formalism, singly-polarized experiments (either
} 
parts of Eq. 1.10 contribute to the cross-section. For an electron beam with lefthanded or right-handed helicity incident upon a target that is polarized along some vector. $\vec{N}$. the cross-section has the form

$$
\frac{d^{2} \sigma_{\vec{N}}^{L(R)}}{d \Omega d E^{\prime}}=\frac{d^{2} \sigma_{\text {unpol. }}}{d \Omega d E^{\prime}} \pm \frac{d^{2} \sigma_{\text {pol. }}}{d \Omega d E^{\prime}} .
$$

where $\frac{d^{2} \sigma_{\text {pol }}}{d \Omega d E^{\prime}}$ is the spin-dependent part of the cross-section. and the sign is positive for left-handed electrons and negative for right-handed electrons. Just as the unpolarized DIS cross-section depends upon two structure functions. $H_{1}\left(\nu . Q^{2}\right)$ and $W_{2}\left(\nu, Q^{2}\right)$, which become $F_{1}(x)$ and $F_{2}(x)$ in the Bjorken limit. the polarized crosssection depends upon two polarized structure functions. $G_{r_{1}}\left(\nu \cdot Q^{2}\right)$ and $G_{2}\left(\nu, Q^{2}\right)$. which. in the Bjorken limit, scale as [1]

$$
\begin{aligned}
\frac{\nu}{M} G_{1}\left(\nu, Q^{2}\right) & \rightarrow g_{1}(x) . \\
\frac{\nu^{2}}{M^{2}} G_{2}\left(\nu, Q^{2}\right) & \rightarrow g_{2}(x) .
\end{aligned}
$$

The difference between the polarized DIS cross-section for left-handed electrons (electron spin anti-aligned with electron momentum) incident on two target polarization states, with the nucleon polarization vector at an angle of $a$ and $a+\pi$ with respect to the electron momentum. is

$$
\begin{aligned}
& \frac{d^{2}[\sigma(\alpha)-\sigma(\alpha+\pi)]}{d \Omega d E^{\prime}}= \\
& \frac{8 \pi^{2}}{Q^{2}}\left\{\cos \alpha\left[\left(1-\frac{y}{2}-\frac{y^{2} \gamma^{2}}{4}\right) g_{1}\left(x \cdot Q^{2}\right)-\frac{y}{2} \gamma^{2} g_{2}\left(x \cdot Q^{2}\right)\right]\right. \\
& \left.\quad-\sin \alpha \cos \phi \gamma \sqrt{1-\frac{y}{2}-\frac{y^{2} \gamma^{2}}{4}}\left[\frac{y}{2} g_{1}\left(x \cdot Q^{2}\right)+g_{2}\left(x \cdot Q^{2}\right)\right]\right\} .
\end{aligned}
$$

where $\alpha$ is the angle between the electron momentum vector and the nucleon spin vector, $\phi$ is the angle between the $k-S$ plane and the $k-k^{\prime}$ plane (see Fig. 1.3).

only the leptons or only the nucleons are polarized) have only the contribution from the unpolarized cross-section. The formalism of Eq. 1.10 neglects flavor-changing interactions (which will not be discussed in this work) and also neglects the (parity-violating) electroweak asymmetry due to interference between the $\gamma^{*}$ and $Z_{0}$ exchange terms; the electroweak asymmetry only depends upon the lepton polarization. The measured asymmetries require a small correction (about $2-4 \%$ relative) due to the electroweak asymmetry; this correction will be discussed in Section 5.6.1. 


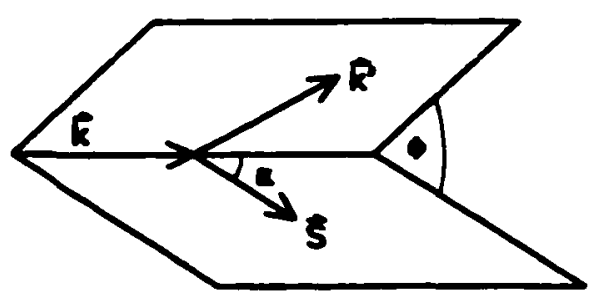

Figure 1.3: Diagram of the polarized DIS geometry. showing the angle between the scattering plane and the polarization plane, $\phi$, and the angle between the nuclear polarization and the electron momentum, $\alpha$.

$y=\left(P_{\mu} q^{\mu}\right) /\left(P_{\mu} k^{\mu}\right)=\nu / E$, and $\gamma=2 M x / \sqrt{Q^{2}}$ [2]. Two different values of $\alpha$ must be used to extract $g_{1}$ and $g_{2}$ from the experimental data. The difference between the cross-sections for left-handed and right-handed electrons $\left(\sigma^{(L)}-\sigma^{(R)}\right)$ results in the same formula as Eq. 1.35, for a fixed value of the angle $\alpha$.

By measuring the scattering rate asymmetry.

$$
\begin{aligned}
& A_{\vec{N}}=\frac{\frac{d^{2} \sigma^{L}}{d \Omega d E^{\prime}}-\frac{d^{2} \sigma^{R}}{d \Omega d E^{\prime}}}{\frac{d^{2} \sigma^{L}}{d \Omega d E^{\prime}}+\frac{d^{2} \sigma^{R}}{d \Omega d E^{\prime}}} . \\
& =\frac{\frac{d^{2} \sigma \xi^{\xi} D}{d \Omega d E^{\prime}}}{\frac{d^{2} \sigma^{0}}{d \Omega d E^{\prime}}} .
\end{aligned}
$$

the spin-dependent cross-sections may be determined in terms of the unpolarized cross-section. Additionally, by measuring the asymmetry instead of the cross-section difference, some of the systematic effects cancel. such as uncertainties in the spectrometer acceptance.

The two asymmetries used to extract $g_{1}$ and $g_{2}$ in E1.5.5 are the parallel asymmetry, $A_{\|}$, in which $\alpha=0$ (or $\pi$ ), and the perpendicular asymmetry. $A_{\perp}$. with $\alpha= \pm \frac{\pi}{2}$. For $A_{\|}, \alpha=0$ and $\phi=0$. For $A_{\perp}, \alpha= \pm \frac{\pi}{2}$. thus only the second set of terms in Eq. 1.35 contribute to the asymmetry, and this set of terms has the largest contribution for $\phi=0$ or $\phi=\pi$, so the largest $A_{\perp}$ occurs for the nuclear polarization vector parallel to the scattering plane. 


$$
\begin{aligned}
D & =\left(1-E^{\prime} \epsilon / E\right) /(1+\epsilon R) \\
\eta & =\epsilon \sqrt{Q^{2}} /\left(E-E^{\prime} \epsilon\right) \\
d & =D \sqrt{2 \epsilon /(1+\epsilon)} \\
\zeta & =\eta(1+\epsilon) / 2 \epsilon
\end{aligned}
$$

Table 1.2: Kinematic terms used in the relationships between $A_{\| 1} \cdot A_{1} . A_{1}$ and $A_{2}$.

We can schematically write the asymmetries.

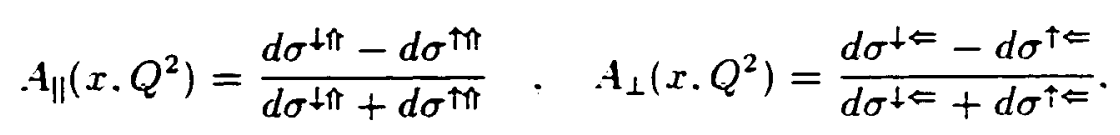

where $d \sigma$ represents the differential cross-section for left-handed $(\downarrow)$ or right-handed $(\uparrow)$ electrons incident upon a target with nuclear polarization aligned with $\alpha=0$

$(\Uparrow)$ or with the nuclear polarization aligned with $\alpha=\frac{\bar{z}}{2}$ and $O=0(\Leftarrow)[2 \tau]$. In terms of the structure functions $g_{1}$ and $g_{2}$.

$$
\begin{aligned}
& A_{\|}\left(x, Q^{2}\right)=f\left[g_{1}\left[E+E^{\prime} \cos \theta\right]-\frac{Q^{2}}{\nu} g_{2}\right] . \\
& A_{\perp}\left(x, Q^{2}\right)=f E^{\prime} \sin \theta\left[g_{1}+\frac{2 E}{\nu} g_{2}\right] .
\end{aligned}
$$

where

$$
f=\frac{1}{F_{1}\left(x, Q^{2}\right)} \frac{1}{\nu} \frac{1-\epsilon}{1+\epsilon R\left(x \cdot Q^{2}\right)}
$$

contains the unpolarized structure functions $F_{1}\left(x, Q^{2}\right)$ and $R\left(x, Q^{2}\right)$ of the nucleon $[2 \tau]$, and

$$
\epsilon=\left[1+2\left(1+\nu^{2} / Q^{2}\right) \tan ^{2} \frac{\theta}{2}\right]^{-1}
$$

is the relative flux of longitudinal virtual photons [3].

Instead of discussing asymmetries in terms of the electron polarization. the virtual photon absorption asymmetries are related to the electron scattering asymmetries by

$$
\begin{aligned}
& A_{\|}\left(x, Q^{2}\right)=D\left(A_{1}+\eta A_{2}\right), \\
& A_{\perp}\left(x, Q^{2}\right)=d\left(A_{2}-\zeta A_{1}\right) .
\end{aligned}
$$


where $D, \eta, d$. and $\zeta$ are shown in Table $1.2[2 \pi]$. The virtual photon absorption asymmetries are

$$
\begin{aligned}
& \boldsymbol{A}_{1}\left(x, Q^{2}\right)=\frac{d \sigma_{1 / 2}-d \sigma_{3 / 2}}{d \sigma_{T}} . \\
& \boldsymbol{A}_{2}\left(x, Q^{2}\right)=\frac{d \sigma_{L T}}{d \sigma_{T}} .
\end{aligned}
$$

where $d \sigma_{1 / 2}$ and $d \sigma_{3 / 2}$ are the photo-absorption cross-sections for transverse polarized photons with total helicity between the photon and nucleon respectively $1 / 2$ and $3 / 2 . d \sigma_{L T}$ is the interference term between the longitudinal and transverse photon-nucleon amplitudes. and $d \sigma_{T}=d \sigma_{1 / 2}+d \sigma_{3 / 2}$ is the total photo-absorption cross-section for transverse polarized photons [28]. Eq. 1.43 and 1.44 may be inverted to give the virtual photon asymmetries.

$$
\begin{aligned}
& A_{1}\left(x \cdot Q^{2}\right)=\frac{A_{\| l}\left(x \cdot Q^{2}\right)}{D(1+\eta \zeta)}-\frac{\eta \cdot A_{\perp}\left(x \cdot Q^{2}\right)}{d(1+\eta \zeta)} \\
& A_{2}\left(x \cdot Q^{2}\right)=\frac{\zeta A_{\|}\left(x \cdot Q^{2}\right)}{D(1+\eta \zeta)}-\frac{A_{\perp}\left(x \cdot Q^{2}\right)}{d(1+\eta \zeta)} .
\end{aligned}
$$

The relations between the virtual photon absorption asymmetries and the spin structure functions are

$$
\begin{aligned}
& A_{1}\left(x \cdot Q^{2}\right)=\frac{g_{1}\left(x \cdot Q^{2}\right)-\gamma^{2} g_{2}\left(x \cdot Q^{2}\right)}{F_{1}\left(x \cdot Q^{2}\right)} \\
& A_{2}\left(x, Q^{2}\right)=\frac{\gamma\left(g_{1}\left(x \cdot Q^{2}\right)+g_{2}\left(x \cdot Q^{2}\right)\right)}{F_{1}\left(x, Q^{2}\right)}
\end{aligned}
$$

where $\gamma^{2}=Q^{2} / \nu^{2}=4 M^{2} x^{2} / Q^{2}[27]$. Notice that for high $Q^{2} . \gamma^{2}$ is small. and $A_{1} \approx g_{1} / F_{1}$. Inverting these relations and using Eq. 1.22 to replace $F_{1}$ with $F_{2}$ and $R$ yields,

$$
\begin{aligned}
& g_{1}\left(x, Q^{2}\right)=\frac{F_{2}\left(x, Q^{2}\right)}{2 x\left[1+R\left(x, Q^{2}\right)\right]}\left[A_{1}\left(x, Q^{2}\right)+\gamma A_{2}\left(x, Q^{2}\right)\right] \\
& g_{2}\left(x, Q^{2}\right)=\frac{F_{2}\left(x, Q^{2}\right)}{2 x\left[1+R\left(x, Q^{2}\right)\right]}\left[A_{2}\left(x, Q^{2}\right) / \gamma-A_{1}\left(x, Q^{2}\right)\right]
\end{aligned}
$$


The spin structure functions, $g_{1}$ and $g_{2}$, may be described in terms of the parallel and perpendicular deep inelastic electron scattering asymmetries. $A_{\|}$and $A_{\perp}$ [ [28]

$$
\begin{aligned}
g_{1}\left(x, Q^{2}\right)= & \frac{F_{1}\left(x, Q^{2}\right)}{D^{\prime}}\left[A_{\|}\left(x, Q^{2}\right)+A_{\perp}\left(x \cdot Q^{2}\right) \tan \frac{\theta}{2}\right] . \\
g_{2}\left(x, Q^{2}\right)= & \frac{F_{1}\left(x \cdot Q^{2}\right) y}{D^{\prime} 2 \sin \theta} \\
& {\left[\frac{E+E^{\prime} \cos \theta}{E^{\prime}} \cdot A_{\perp}\left(x, Q^{2}\right)-\sin \theta \cdot A_{\|}\left(x, Q^{2}\right)\right] . }
\end{aligned}
$$

where the depolarization factor

$$
D^{\prime}=\frac{(1-\epsilon)(2-y)}{y\left[1+\epsilon R\left(x \cdot Q^{2}\right)\right]}
$$

corrects for the difference in polarizations of the virtual photon and the electron.

\subsection{Spin Structure Function Evolution}

Just as $F_{1}$ has a simple interpretation in the naive quark parton model given by $\mathrm{Eq} \cdot 1.24$, the spin structure function $g_{1}$ also has a simple interpretation.

$$
g_{1}=\frac{1}{2} \sum_{i=u, d . s} \epsilon_{i}^{2} \Delta q_{i}(x)
$$

where the polarized quark distributions are the difference in the quark distributions for quark spins aligned and anti-aligned with the nucleon spin. $\Delta q_{i}(x)=q_{i}^{\uparrow}(x)-$ $q_{i}^{\downarrow}(x)$; the quark distributions which appear in $F_{1}$ are the sum $\left(q_{i}(x)=q_{i}^{\dagger}(x)+q_{i}^{\downarrow}(x)\right)$. This leads to the naive expectation that $g_{1} / F_{1}$ is independent of $Q^{2}$. although there is no theoretical reason for this to hold; this naive expectation of the $Q^{2}$ dependence of $g_{1}$ following that of $F_{1}$ is approximately followed by the data. but deviations from this picture do occur.

The spin structure functions depend upon the parton distributions in the nucleon. so by determining the variation of the structure functions over a wide range in $x$ and $Q^{2}$, the polarized parton distributions may be constrained. The departure of the spin structure functions from Bjorken scaling arises from the evolution of 
the parton distribution functions in $Q^{2}$, and from the inclusion of terms of higher order in QCD. Two techniques may be used to evaluate the $Q^{2}$ dependencies: phenomenological modeling and fitting using next-to-leading-order (NLO) perturbative quantum chromodynamics ( $\mathrm{PQCD}$ ). The E15:5 collaboration used both techniques during the physics analysis of our results.

The form of the phenomenological model is partially dictated by the $Q^{2}$ behavior expected for various types of scaling violation effects. The simplest picture. as described earlier in this section, is that $g_{1} / F_{1}$ would be independent of $Q^{2}$. Additional $Q^{2}$ dependence of $g_{1} / F_{1}$ might arise from other effects, such as different evolution of the polarized distributions under perturbative QCD. or contributions of higher twist ${ }^{2}$. The empirical fits are done using data of either the extracted virtual photon asymmetry, $A_{1}$, or the ratio of the polarized and unpolarized structure functions, $g_{1} / F_{1}$. The forms of $Q^{2}$ dependence used in this type of modeling are: no additional $Q^{2}$ dependence; additional dependence as $1 / Q^{2}$. which arises from the higher twist effects: and dependence as $\ln \left(1 / Q^{2}\right)$. which arises from different evolution in perturbative QCD [28].

In perturbative QCD, the structure function $g_{1}$ is described in terms of the quark $\left(\Delta q_{i}\right)$ and gluon $(\Delta G)$ polarized parton distributions. analogous to Eq. 1.26.

$$
g_{1}\left(x, Q^{2}\right)=\frac{1}{2} \sum_{i}^{N_{f}} \epsilon_{i}^{2}\left(C_{q} \odot \Delta q_{i}+\frac{1}{N_{f}} C_{g} \odot \Delta C_{i}\right) .
$$

where the sum is over the active quark flavors $\left(N_{f}\right)$. and the Wilson coefficients are series in powers of $\alpha_{s}$ :

$$
C_{q, G}(x)=C_{q, G}^{(0)}(x)+\frac{\alpha_{s}}{2 \pi} C_{q, G}^{(1)}(x)+\ldots
$$

The polarized parton distributions are parameterized in terms of the unpolarized

\footnotetext{
${ }^{2}$ Higher twist (discussed more fully in Section 1.6) operators include such terms as quark-gluon correlations, and give rise to deviations from the leading-order expectations.
} 
parton distributions, using the form

$$
\Delta q_{i}\left(x, \mu^{2}\right)=A_{i} x^{\alpha_{i}} q_{i}\left(x, \mu^{2}\right),
$$

where $\Delta q_{i}$ and $q_{i}$ are to be taken as either the quark or gluon distributions. The parameterized distributions are evolved from the low initial scale of $Q^{2}=\mu^{2}$ to the experimental $Q^{2}$ using the DGLAP equations, then fit to the data to determine the polarized parton distributions at the input scale. The polarized parton distributions may then be evolved to an average $Q^{2}$ (such as $\left.Q^{2}=5(\mathrm{GeV} / \mathrm{c})^{2}\right)$ and used to evaluate the helicity contribution from the partons or integrals of the structure functions. The integrals of the structure functions enter into sum rules (discussed in Section 1.i) which predict certain behaviors from the structure functions.

The total helicity of the nucleon $\left(\frac{1}{2}\right)$ is given by the helicity contributions from the partons and orbital angular momentum. The nucleon spin sum rule is usually written as,

$$
\frac{1}{2}=\frac{1}{2} \Delta \Sigma+\Delta G+\left\langle L_{z}\right\rangle
$$

where $\Delta \Sigma=\int_{0}^{1} \Delta \Sigma\left(x, Q_{0}^{2}\right) d x$ is the quark polarization, $\Delta G=\int_{0}^{1} \Delta G\left(x, Q_{0}^{2}\right) d x$ is the gluon polarization, and $\left\langle L_{z}\right\rangle$ is the contribution to the nucleon spin from the orbital angular momentum; the integrals must be done with $Q^{2}$ constant as is represented by $Q_{0}^{2}$ in Eq. 1.60 .

\subsection{Previous Polarized DIS Experiments}

Experiment E155 was preceded by several polarized DIS measurements at SLAC, CERN, and DESY; the experiments are listed in Table 1.3. The CERN measurements (EMC, SMC) were done at high $Q^{2}$. and could reach lower $x$. but had poorer statistical precision. The SLAC measurements (E80, E130. E142. E143). had a more limited kinematic range. but achieved higher statistics. The measurements at HERMES had similar kinematics to the SLAC experiments. The E1.55 


\begin{tabular}{|c|c|c|}
\hline Experiment & Beam & Target Material(s) \\
\hline \multicolumn{3}{|c|}{ SLAC Measurements } \\
\hline E80[29] & $\mathrm{e}^{-} ; 6-13 \mathrm{GeV}$ & Butanol \\
\hline $\mathrm{E} 130[30]$ & $\mathrm{e}^{-}: 23 \mathrm{GeV}$ & Butanol \\
\hline $\mathrm{E} 142[31]$ & $\mathrm{e}^{-}: 19.4 .22 .7 \cdot 25.5 \mathrm{GeV}$ & ${ }^{3} \mathrm{He}$ gas \\
\hline E143[28] & $\mathrm{e}^{-}: 9.7,16.2,29.1 \mathrm{GeV}$ & $\mathrm{NH}_{3} \& \mathrm{ND}_{3}$ \\
\hline$E 154[32.33]$ & $\mathrm{e}^{-}: 48.3 \mathrm{GeV}$ & ${ }^{3} \mathrm{He}$ gas \\
\hline \multicolumn{3}{|c|}{ CERN Measurements } \\
\hline $\mathrm{EMC}[34,3.5]$ & $\mu^{+}: 100,120 . \& 200 \mathrm{GeV}$ & Ammonia \\
\hline $\mathrm{SMC}[36,37]$ & $\mu^{+} ; 100-190 \mathrm{GeV}$ & Butanol. Deut. Butanol. $\mathrm{NH}_{3}$ \\
\hline \multicolumn{3}{|c|}{ DESY Measurements } \\
\hline HERMES[38, 39] & $\mathrm{e}^{+} ; \sim 27.5 \mathrm{GeV}$ & ${ }^{3} \mathrm{He} \& \mathrm{H}_{2}$ gas \\
\hline
\end{tabular}

Table 1.3: A summary of polarized DIS experiments before E155. The butanol. ammonia $\left(\mathrm{NH}_{3}\right)$, and $\mathrm{H}_{2}$ targets were used to measure the proton spin structure functions. The deuterated butanol and deuterated ammonia $\left(\mathrm{ND}_{3}\right)$ targets were used to measure the spin structure functions of the deuteron, which can be used to extract the neutron structure functions. The ${ }^{3} \mathrm{He}$ target provided a different way to measure the neutron spin structure functions.

(and the previous E154 measurement) kinematics fit into the region in $x$ and $Q^{2}$ between the CERN measurements and the earlier SLAC and DESY measurements.

The first two experiments, E80 and E130, did not cover a sufficiently large $x$ range to be able to evaluate $\Delta \Sigma$ well. The EMC experiment extended the measured region to lower $x$. Using the assumption that $g_{1}$ was independent of $Q^{2}$ over the measured region. and extrapolating to determine the contribution from the unmeasured regions, the EMC collaboration found the first moment of the E80+E130+E.IC $g_{1}$ data to be [3.5],

$$
\int_{0}^{1} g_{1}^{p}(x) d x=0.126 \pm 0.010 \pm 0.015
$$

From this. the EMC collaboration determined a value for $\Delta \Sigma$ of $\Delta \Sigma=0.120 \pm$ $0.094 \pm 0.138[35]$, which is inconsistent with the Ellis-.Jaffe prediction (in which $\Delta s=0$ ) of $\Delta \Sigma=0.60 \pm 0.12[40]$. In both the EMC determination and the EllisJaffe prediction of $\Delta \Sigma$, the gluons were assumed not to contribute to $g_{1}$.

This result was known as the "spin crisis", and led to extensive efforts to understand the spin structure functions. The subsequent experiments have measured 
different kinematic ranges to be able to use NLO fitting (as presented in Section 1.4) to determine the quark and gluon contributions to nucleon spin structure. The E1.54 collaboration has done a NLO fit in the $\overline{\mathrm{MS}}$ scheme, evaluated at $Q^{2}=5$ $(\mathrm{GeV} / \mathrm{c})^{2}$. with the results

$$
\begin{aligned}
& \Delta \Sigma=0.20_{-0.06-0.05-0.01}^{+0.05+0.04+0.01} . \\
& \Delta G=1.8_{-0.7-0.5}^{+0.6+0.4},+0.1
\end{aligned}
$$

where the errors are statistical, systematic, and theoretical [41].

The goals of E15.5 were to provide high precision measurements of $g_{1}$ for the proton and deuteron covering the range in $Q^{2}$ between the preceding data at low and high $Q^{2}$. These high precision measurements would improve our knowledge of the structure functions and provide greater constraint in the NLO fitting. producing more accurate extractions of $\Delta \Sigma$ and $\Delta G$. In the process of making these high precision measurements of $g_{1}$, E1.55 would also make lower precision measurements of the $g_{2}$ structure function. which is more sensitive to the effects of higher twist in the nucleon.

\subsection{Operator Product Expansion}

The polarized structure functions may also be evaluated using the Operator Product Expansion (OPE). In the OPE. the structure functions are written in terms of renormalized operators of increasing twist [42]. The twist of an operator is the difference between the dimension and spin of the operator [1]: the smallest possible value of twist for QCD operators is twist-2 [43]. The moments of the structure functions $g_{1}$ and $g_{2}$ are related to the twist-2 and twist-3 reduced matrix elements. $a_{n}$ and $d_{n}$. and higher twist terms suppressed by factors of $1 / \sqrt{Q^{2}}$ [42]. Neglecting quark mass terms [42], the moments are

$$
\int_{0}^{1} x^{n} g_{1}\left(x, Q^{2}\right) d x=\frac{1}{2} a_{n}+O\left(M^{2} / Q^{2}\right) . n=0,2, \ldots
$$




$$
\int_{0}^{1} x^{n} g_{2}\left(x, Q^{2}\right) d x=\frac{1}{2} \frac{n}{n+1}\left(d_{n}-a_{n}\right)+O\left(M^{2} / Q^{2}\right) . n=2.4 \ldots \ldots
$$

The structure function $g_{2}$ can be written as $g_{2}=g_{2}^{w w}+\overline{g_{2}}$. where

$$
g_{2}^{W W}\left(x, Q^{2}\right)=-g_{1}\left(x \cdot Q^{2}\right)+\int_{x}^{1} \frac{g_{1}\left(y, Q^{2}\right)}{y} d y
$$

is the leading twist-2 contribution derived by Wandzura and Wilczek [44]. The term $\overline{g_{2}}$ is interpreted dominantly as due to twist-3 terms from quark-gluon correlations [4:2]. Then the twist-3 reduced matrix elements. $d_{n}$. are

$$
d_{n}=2 \frac{n+1}{n} \int_{0}^{1} x^{n} \overline{g_{2}}\left(x, Q^{2}\right) d x . n=2,4 \ldots
$$

allowing the measurement of deviations from $g_{2}^{w w}[42]$.

\section{$1 . \bar{T}$ Sum Rules}

The Bjorken sum rule [45] is a fundamental prediction, deriving from current algebra and isospin symmetry, which relates the difference in the first moments of $g_{1}$ for the proton and neutron to the ratio of nucleon axial-vector and vector couplings measured in neutron beta decay. For finite $Q^{2}$ there are QCD corrections. which yield

$$
\begin{aligned}
\Gamma_{1}^{p-n}=\int_{0}^{1} g_{1}^{p}-g_{1}^{n} d x= & \frac{1}{6} \frac{g_{A}}{g_{V}}\left[1-\frac{\alpha_{s}\left(Q^{2}\right)}{\pi}\right. \\
& \left.-3.58\left(\frac{\alpha_{s}\left(Q^{2}\right)}{\pi}\right)^{2}-20.22\left(\frac{\alpha_{s}\left(Q^{2}\right)}{\pi}\right)^{3}\right] .
\end{aligned}
$$

evaluated in the next-next-to-leading order where only the three light quark flavors are taken to contribute [46]. Taking $\alpha_{S}\left(M_{Z}^{2}\right)=0.119 \pm 0.002[4]$. which yields the evaluation of $\alpha_{S}\left(5(\mathrm{GeV} / c)^{2}\right)=0.29 \pm 0.02$, the Bjorken sum rule prediction (at $\left.Q^{2}=5(\mathrm{GeV} / \mathrm{c})^{2}\right)$ is $[47]$

$$
\Gamma_{1}^{p-n}=0.182 \pm 0.005
$$

The Burkhardt-Cottingham sum rule [48] for large $Q^{2}$.

$$
\int_{0}^{1} d x g_{2}(x)=0
$$


was derived from virtual Compton scattering dispersion relations.

The Efremov-Leader-Teryaev sum rule [49] relates the valence quark contributions of $g_{1}$ and $g_{2}$,

$$
\int_{0}^{1} d x x\left[g_{1}^{v^{\prime}}(x)+2 g_{2}^{v}(x)\right]=0
$$

where $g_{1}^{V}$ and $g_{2}^{V}$ are the valence quark contributions to the structure functions. By assuming the sea quarks are the same in protons and neutrons, the sum rule becomes $[42]$

$$
\int_{0}^{1} d x x\left[g_{1}^{p}(x)-g_{1}^{n}(x)+2 g_{2}^{p}(x)-2 g_{2}^{n}(x)\right]=0
$$




\section{CHAPTER 2}

\section{E155 EXPERIMENTAL EQUIPMENT}

Measurement of the polarized deep inelastic electron-scattering asymmetries requires a polarized electron beam, a target containing polarized nucleons, and detector systems, all sketched in Fig. 2.1. Equation 2.1 shows the relation between the counting rate asymmetry, $A^{\text {meas }}$, and the uncorrected deep inelastic scattering asymmetry, $A^{\text {uncorr }}$,

$$
A^{\text {meas }}\left(x, Q^{2}\right)=\left[\frac{N^{\dagger} / Q^{\downarrow}-N^{\dagger} / Q^{\dagger}}{N^{\dagger} / Q^{\dagger}+N^{\dagger} / Q^{\dagger}}\right]\left(x, Q^{2}\right)=C_{1} f P_{B} P_{T} A^{\text {uncorr }}\left(x, Q^{2}\right) \text {. }
$$

The measured asymmetry, $A^{\text {meas }}$, is the difference in the scattering rate, $N / Q$ (number of scattering events per incident beam charge), for electron helicity antiparallel $(\downarrow)$ and parallel $(\uparrow)$ to the target spin. This counting rate asymmetry, $A^{\text {meas }}$, is proportional to the uncorrected deep inelastic asymmetry, $A^{\text {uncorr }}$, where the constants of proportionality are the dilution factor $(f)$, the nuclear correction $\left(C_{1}\right)$, and the beam and target polarizations $\left(P_{B}, P_{T}\right)$. This uncorrected DIS asymmetry, $A^{\text {uncorr }}$, excludes corrections such as rate dependence, contaminant events, and radiative effects. $A^{\text {uncorr }}$ is also not corrected for an additional additive nuclear correction

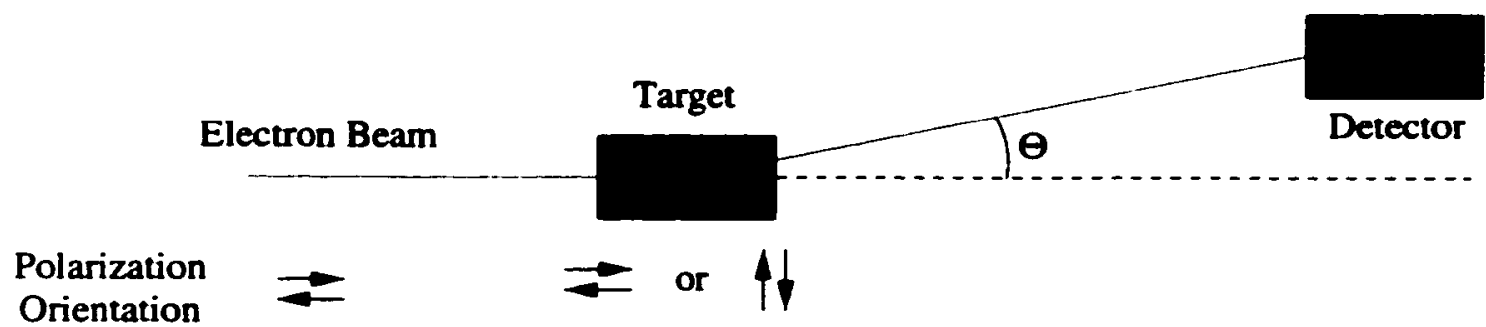

Figure 2.1: A block diagram of a polarized DIS experiment showing a longitudinally polarized electron beam incident upon a target polarized either longitudinally or transverse to the electron beam. Some of the electrons are scattered into the detector system. 
term. The determination of the fully corrected DIS asymmetry will be discussed in Section 5.6. The dilution factor is the fraction of events originating in the polarizable target nucleons (protons or deuterons. depending on target material). The nuclear correction term is due to scattering from other polarized nuclei in the target material (such as ${ }^{15} \mathrm{~N}$ in the proton target, or ${ }^{6} \mathrm{Li}$ in the deuteron target).

Since the scattering asymmetry for high energy electrons is small and the statistical error scales as $1 / \sqrt{N}$, an intense electron beam, a large number of target nuclei, large acceptance detectors, and a long running period are desirable to collect high statistics on the measured asymmetry. The beam intensity, target thickness and detector acceptances are limited by the need to balance reductions in statistical errors with increases in systematic errors or corrections. The statistical uncertainties. $\delta A^{\text {meas }}$ and $\delta A^{u n c o r r}$, of the measured and extracted DIS asymmetry are related to each other by the same relation as shown in Eq. 2.1. Thus. high polarizations of the electron beam and target nuclei, and a high dilution factor are desirable to minimize uncertainties on the extracted DIS asymmetry. The goals in experimental design are to have an intense. highly polarized electron beam. a dense. highly polarized target containing a large fraction of the polarized target nuclei. and spectrometers with wide acceptances that have the ability to identify the energy and angle (hence $Q^{2}$ and $x$ ) of the scattered electrons.

\subsection{The SLAC Polarized Electron Beam}

The SLAC accelerator, which is capable of accelerating electrons up to energies of $50 \mathrm{GeV}$ at a pulse (or spill) rate of $120 \mathrm{~Hz}$. consists of a two-mile long series of copper microwave resonant cavities. During the E15.5 longitudinal asymmetry measurement, the accelerator delivered a $48.3 \mathrm{GeV}$ electron beam with $(81.0 \pm$ $2.0) \%$ polarization into End Station A (ESA). The beam pulse was about $300 \mathrm{~ns}$ in duration, containing between $1 \times 10^{9}$ and $5 \times 10^{9}$ electrons, with the typical beam 
current being about $3 \times 10^{9}$ electrons per pulse. The beam current was chosen so that the counting rates in the detectors did not exceed the capability of the electronics. During the transverse asymmetry measurement. the beam energy was reduced to $38.8 \mathrm{GeV}$, the pulse length was increased to about $400 \mathrm{~ns}$, and the beam current was reduced to $1.5 \times 10^{9}$ electrons per pulse. At the lower beam energy. the DIS cross-section is increased, and the pion production cross-section is decreased. compared to the $48.3 \mathrm{GeV}$ electron beam. Reducing the beam energy also reduces the soft particle background produced via synchrotron radiation emitted by the beam in the chicane and target magnets (see Section 2.2.3). The energy emitted by a charged particle traversing a magnetic field scales as the square of the momentum. therefore reducing the beam energy reduces the photon flux around the target. Also. with the lower beam energy, the accelerator could deliver a longer pulse. thereby reducing the instantaneous beam intensity. Nevertheless. because of the different beam optics in the transverse mode. the amount of background events increased. requiring a reduction in the total beam current.

The SLAC polarized electron source (illustrated in Fig. 2.2) utilizes photoemission from a strained GaAs cathode illuminated with circularly-polarized laser light [50]. Light from a flash-lamp pumped Ti:Sapphire laser is circularly-polarized by a Pockels cell. Application of positive or negative high voltage to the Pockels cell produced circularly-polarized light of left-handed or right-handed helicity. The sign of the Pockels cell high voltage, which determines the laser helicity. was changed on a pulse to pulse basis using a pseudo-random 32-bit number generator. depicted in Fig. 2.3. The polarization of a pulse is given by the 32nd bit. Then a new zeroth bit is generated by a logical XOR of the 32 nd and 19 th bits. and the bits are shifted up to provide a new polarization bit for the next pulse [2:3].

When the circularly-polarized laser light illuminates the strained Ga.As photocathode, the photoemitted electrons are polarized. The active layer of the cathode 


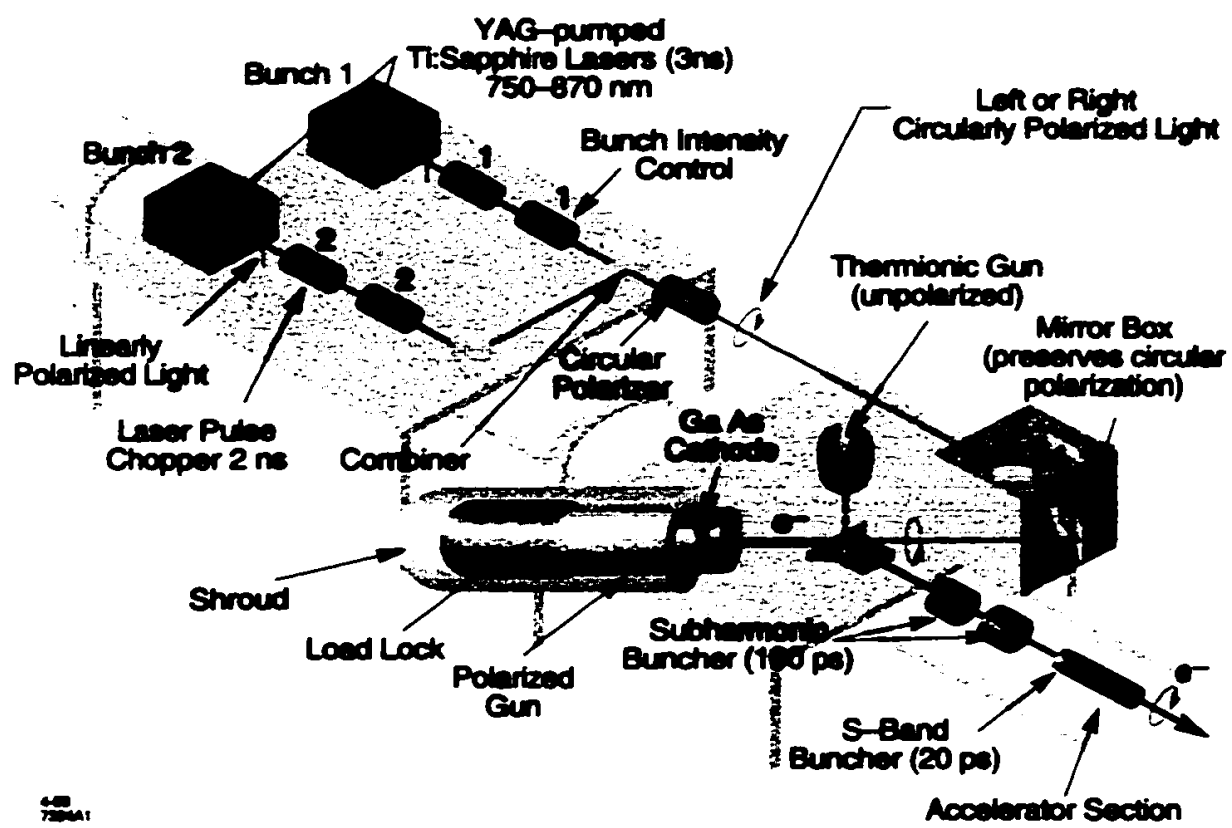

Figure 2.2: Drawing of the SLAC polarized electron source, configured for SLC beam production. For ESA experiments, one of the YAG-pumped Ti:Sapphire lasers is replaced by a flash-lamp pumped Ti:Sapphire laser [50].

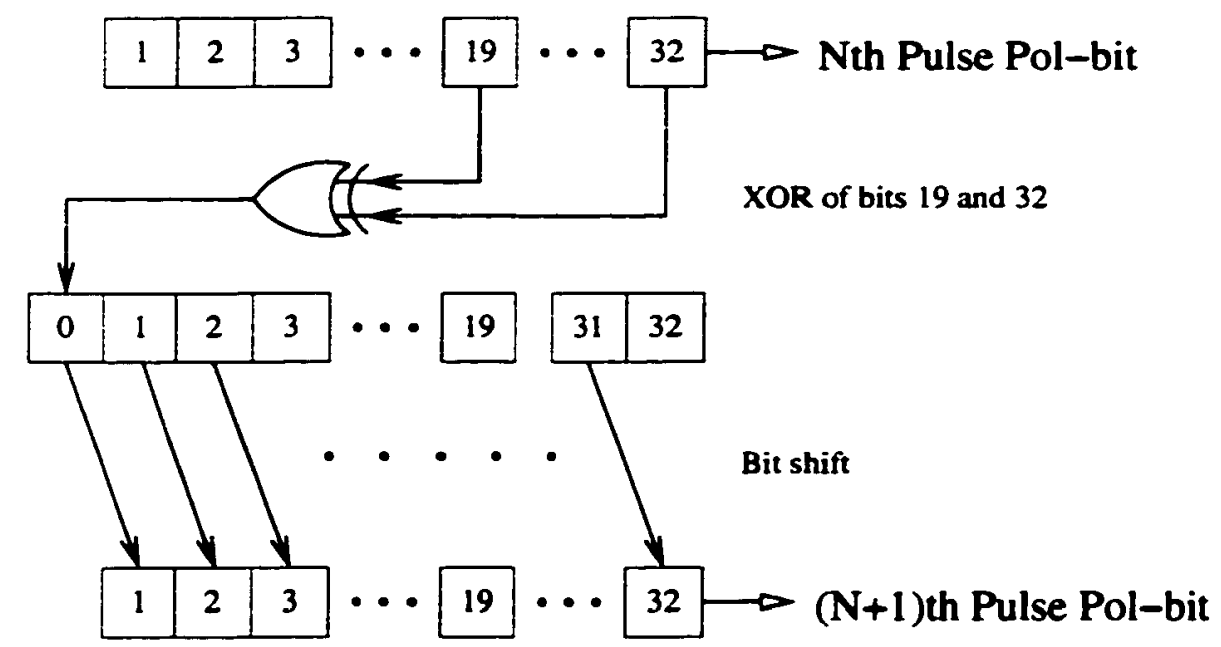

Figure 2.3: Schematic of the algorithm used to determine the polarization bit from the 32 bit register. By recording the polarization bit for 32 consecutive beam pulses; each subsequent bit may be determined using this algorithm. 

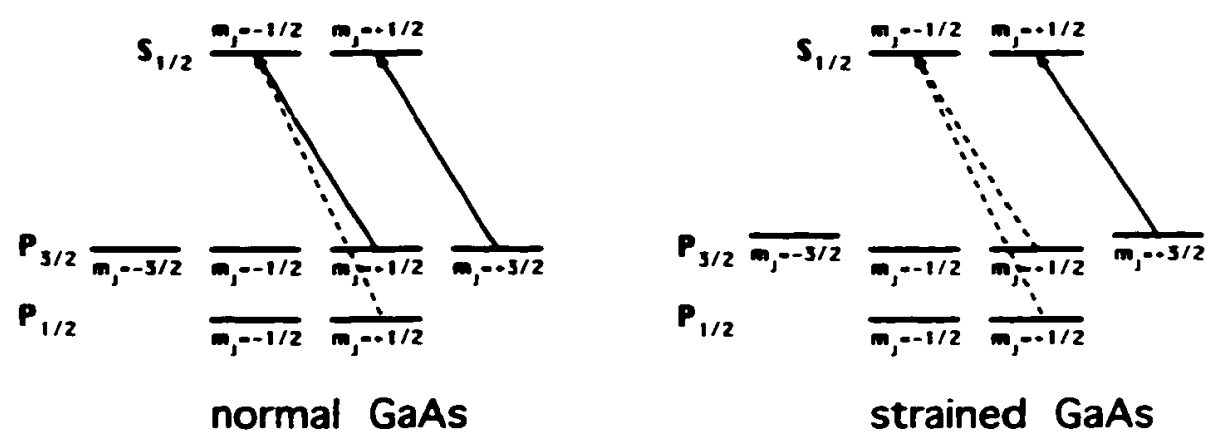

Figure 2.4: Energy level diagram of bulk GaAs and strained GaAs, showing the splitting of the degeneracy in the $P_{3 / 2}$ levels.

is GaAs $0.3 \mu \mathrm{m}$ thick deposited upon a $2.5 \mu \mathrm{m}$ thick layer of $\mathrm{GaAs}_{(0.76)} \mathrm{P}_{(0.24)}[.51]$. The GaAsP layer has a smaller lattice constant than the GaAs. which produces a strain in the GaAs lattice and thus distorts the electron energy bands in the GaAs [51], as shown in Fig. 2.4. In bulk GaAs, the valence band is degenerate between the $m_{j}= \pm 3 / 2$ and $m_{j}= \pm 1 / 2$ substates of the $P_{3 / 2}$ level. When the bulk GaAs is illuminated by circularly-polarized light. electrons are excited into the conduction band from both substates. If negative helicity light were used to illuminate the bulk GaAs, transitions from the $m_{j}=3 / 2$ and $m_{j}=1 / 2$ sublevels would occur. but the transitions from the $m_{j}=3 / 2$ level would be three times more likely, resulting in a mixture of polarization states in the conduction band. and a maximum net electron polarization of $50 \%$. In the strained GaAs, the degeneracy between the $m$-states in the $P_{3 / 2}$ valence band is broken. By illuminating the strained cathode with polarized laser light with a narrow line width. the electrons are selectively excited from the $m_{j}= \pm 3 / 2$ sublevels. This technique yields a theoretical maximum polarization of $100 \%$. but in practice yields polarizations of about $8.5 \%$ [50]. The electrons emitted have the same helicity as the incident light, as they have opposite spin direction. but are emitted opposite the direction of the incident light.

A thin coating of cesium and $\mathrm{NF}_{3}$ is applied to the cathode to create a negative electron affinity surface [52]. The vacuum energy level is reduced below the con- 
duction band in the bulk material, increasing the electron photoemission efficiency. During high current operation the cathode generally requires periodic re-cesiation. however this was unnecessary during E15.5 because the beam currents were low.

The electrons produced by the polarized source are then collected and injected into the linear accelerator. The linear accelerator is 10000 feet long. consisting of a disk-loaded waveguide [53]. The microwave power is provided by a system of about 240 klystrons operating in the S-band $(2856 \mathrm{MHz})$ [54]. The maximum energy of the SLAC accelerator was initially only about $25 \mathrm{GeV}$. Instead of replacing all of the klystrons with more powerful klystrons, the peak power available to the accelerator was increased using the SLAC Energy Development (SLED) system. in which a pair of resonant cavities on each klystron store microwave power during the pulse. then release it into the accelerator by introducing a phase reversal of the input microwave signal. The effect of the storage cavities and phase reversal is that the peak power transmitted to the accelerating cavities is increased at the expense of the length of the microwave power pulse [55]. A further refinement of the SLED system is the Flat SLED (SLEDF) mode, in which a long pulse beam with a flat energy and intensity profile is produced. The SLEDF system uses two additional phase reversals on some of the klystrons to produce a notch in the microwave power delivered to their accelerating cavities. The power which would have been transmitted to the accelerator during the notch is stored, and added to the power delivered after the notch. By this process, the power delivered to the different parts of the beam pulse can be tuned, resulting in a beam energy dispersion of about $0.15 \%$ full width. with lower energy tails at the beginning and end of the pulse [56]. Through adjustment of the relative phases of the microwaves produced by each klystron and the use of the SLEDF system, the electron density and energy of the beam are kept fairly constant over the 400 ns beam pulse. 


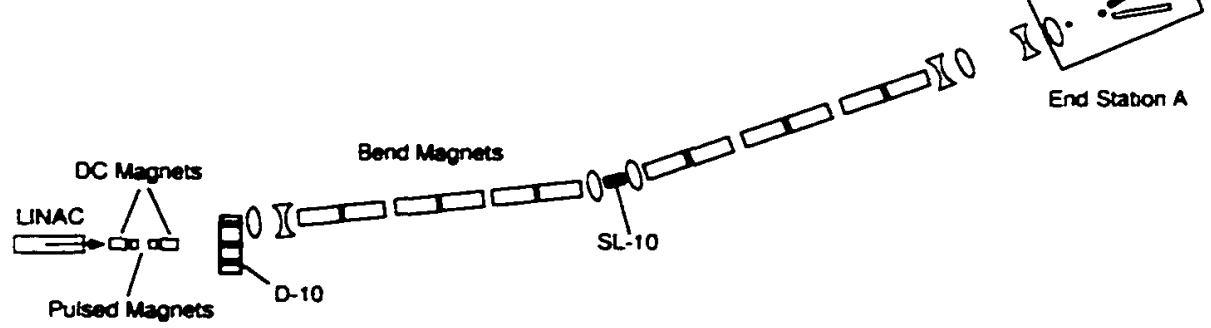

Figure 2.5: Drawing of the A-Line. showing the twelve bend magnets. and the energy defining slits, SL-10.

\subsection{Beam Transport}

As the electrons exit the accelerator, they enter the beam switchyard (BSY). Two pulsed magnets deflect the electrons by $0.5^{\circ}$ from the BSY common beam-line into Beam Line A (A-Line), which is shown in Fig. 2.5. Within the A-Line. twelve identical dipole magnets transport the beam to ESA through an additional bend of $24^{\circ}$ for a total bend of $24.5^{\circ}[5 \pi]$. A thirteenth identical dipole. located in the Main Control Center, is connected in series with the A-Line bend magnets: a flip-coil in this magnet provides a measurement of the central momentum of the A-Line (this is the flip-coil energy). Beam Line $A$ contains several quadrupole and $X$ and $Y$ dipole corrector magnets to control the beam focus and steering through the A-Line. The beam energy and dispersion are controlled by the magnetic strength of the dipoles. and by the setting of the SL-10 slits. The Beam Line A maximum energy width. as set by the Beam Line A slits, was set at $0.8 \%$ full width during E1.5.5.

When an electron travels through a magnetic field. both its momentum and spin vectors undergo rotation. If the magnetic moment of the electron were exactly equal to the Bohr magneton $\left(\mu_{e} / \mu_{B}=g / 2=1\right)$, the spin vector and momentum vector would rotate at the same rate, remaining parallel. However. due to the anomalous magnetic moment of the electron $((g-2) / 2 \neq 1)$, the spin vector precesses with respect to the momentum vector. Equation 2.2 shows the incremental precession angle between the spin vector and the momentum vector, ignoring synchrotron energy 
loss. as a function of energy and incremental bend angle:

$$
\Delta \theta_{\text {precession }}=\frac{\gamma}{2}(g-2) \Delta \theta_{\text {bend }}=\frac{E}{2 m}(g-2) \Delta \theta_{\text {bend }}
$$

where $(g-2) / 2=1159.65 \times 10^{-6}[4] . E$ is the electron energy. $m$ is the electron mass, and $\Delta \theta_{\text {bend }}$ is the total bend angle.

Since the electrons accelerate in the magnetic field. they emit synchrotron radiation, thus losing energy. The synchrotron energy loss depends upon the beam energy, $E$. the bend angle, $\theta_{\text {bend }}$, and the bending radius. $\rho$ :

$$
\Delta E_{\text {synch }}=\frac{2 \theta_{\text {bend }}}{3} \frac{\epsilon^{2}}{\rho}\left(\frac{E}{m}\right)^{4}
$$

where $\epsilon$ is the electron charge. The bending radius of the Beam Line $A$ magnets is 85.927 meters [23]. For an incident beam energy of $48.7 \mathrm{GeV}$. Eq. 2.3 gives a total energy loss through Beam Line $\mathrm{A}$ of $393 \mathrm{MeV}$. resulting in a beam energy after transport of $48.3 \mathrm{GeV}$. Similarly, a beam of $38.8 \mathrm{GeV}$ delivered to ESA would have an initial energy of about $39 \mathrm{GeV}$ and would lose $161 \mathrm{MeV}^{\circ}$ in transport.

The synchrotron energy loss affects the spin precession. since the beam does not have the same energy throughout the bend. By using Eq. 2.3 to describe the beam energy used in Eq. 2.2 as a function of bend angle. the incremental spin precession angle is, to first order in $\theta_{\text {bend }}$.

$$
\frac{d \theta_{\text {precession }}}{d \theta_{\text {bend }}}=\frac{g-2}{2 m}\left(E_{0}-\frac{2 e^{2}}{3 \rho}\left(\frac{E_{0}}{m}\right)^{4} \theta_{\text {bend }}\right)
$$

where $E_{0}$ is the incident beam energy. In further orders of $\theta_{b e n d}$ the $\left(E_{0} / m\right)^{4}$ term would have corrections due to the decreasing beam energy. but at these beam energies $\left(\Delta E_{\text {synch }} / E<0.01\right)$, the corrections are only a few percent modification to that term. Integrating Eq. 2.4 over the bend angle and evaluating vields.

$$
\theta_{\text {precession }}=\int_{0}^{\theta_{\max }} \frac{d \theta_{\text {precession }}}{d \theta_{\text {bend }}} d \theta_{\text {bend }} \text {. }
$$




$$
\begin{aligned}
& \theta_{\text {precession }}=\frac{g-2}{2 m}\left(E_{0} \theta_{\text {bend }}-\frac{\epsilon^{2}}{3 \rho}\left(\frac{E_{0}}{m}\right)^{4} \theta_{\text {bend }}^{2}\right) . \\
& \theta_{\text {precession }}=0.9704 E_{0}-3.4 \times 10^{-8} E_{0}^{4} .
\end{aligned}
$$

where the incident beam energy, $E_{0}$, is in units of $\mathrm{GeV}$. and the final spin precession angle is in radians.

The energy settings of the accelerator are chosen so that the total spin precession angle. given by Eq. 2.7. is an integer multiple of $\pi$. so that the electrons delivered to ESA have the maximum longitudinal polarization. An incident beam energy of $48.759 \mathrm{GeV}$ results in a spin precession of $1.5 \pi$ and a $48.362 \mathrm{GeV}$ beam delivered to ESA [58]. The $38.8 \mathrm{GeV}$ beam delivered to ESA corresponds to a spin precession of $12 \pi$. This energy dependence on the polarization was used as a calibration for the A-Line, by measuring the polarization over a range in energy settings, and fitting the energy dependence to determine the maximum polarization. The polarization measurement technique is discussed in Section 2.2.2.

\subsubsection{The ESA Beam-line Elements}

As the beam passes through ESA, there are numerous pieces of instrumentation for beam containment and evaluation. Only a few of these devices on the ESA beamline are directly used by the experiment. with the remainder used by the accelerator operators in the Main Control Center (MCC). A pair of Helmholtz coils are used to raster the beam location over the target cell, so as to disperse the beam heating effects over the target material. Two roller screens are used during beam tuning to examine the beam spot and the steering of the beam through ESA. A traveling wave beam position monitor provides additional information about the beam steering. A set of toroids measure the beam current in each spill. Two "spill monitors". plastic scintillator paddles located at the beam entrance to ESA and slightly downstream and below the target, serve as a monitor of beam quality during the spill. A final 
measurement of the beam size and position is provided by the foil array. which provides the best visualization of the beam spot at the target. The foil array consists of a set of foils aligned edgewise in the beam path which measure the beam position through secondary electron emission.

The electron beam position on the target is rastered so that the radiation dose and the depolarization effects due to beam heating are spread evenly over the target material. The rastering is done by a set of magnets located at the end of the A-line. just before the beam enters ESA. The beam spot was moved to a new raster position for every spill. The raster pattern had a nominal radius of $9.7 \mathrm{~mm}$ and a step size of $0.3 \mathrm{~mm}$ at the target.

Two fluorescent roller screens, mylar screens coated with zinc sulfide $(\mathrm{ZnS})$. were used during beam tuning to observe the beam spot size and steering in ESA. When placed in the path of the beam, the roller screens fluoresced where struck by the beam, showing the shape and position of the beam. The beam could be steered to center the beam spot with respect to projected cross-hairs on each screen. Television cameras transmitted pictures of the roller screens to Counting House $A(\mathrm{CHA})$ and to $\mathrm{MCC}$. Since the interaction of the beam with the roller screens generates unacceptable backgrounds, they were only used during beam tuning.

The traveling wave beam position monitor (TWBPM) is located just upstream of the target. providing a non-invasive beam position measurement just before the target. The TWBPM consisted of two radio-frequency cavities. one producing a signal proportional to the vertical displacement of the beam from the center. and one producing a signal proportional to the horizontal displacement of the beam from the center [23]. This device is primarily used in the beam position feedback system, and is not used in the main data analysis, although it was used during beam positioning and focusing tests at the beginning of the experiment.

The beam current is monitored using two toroids. Each toroid consists of 


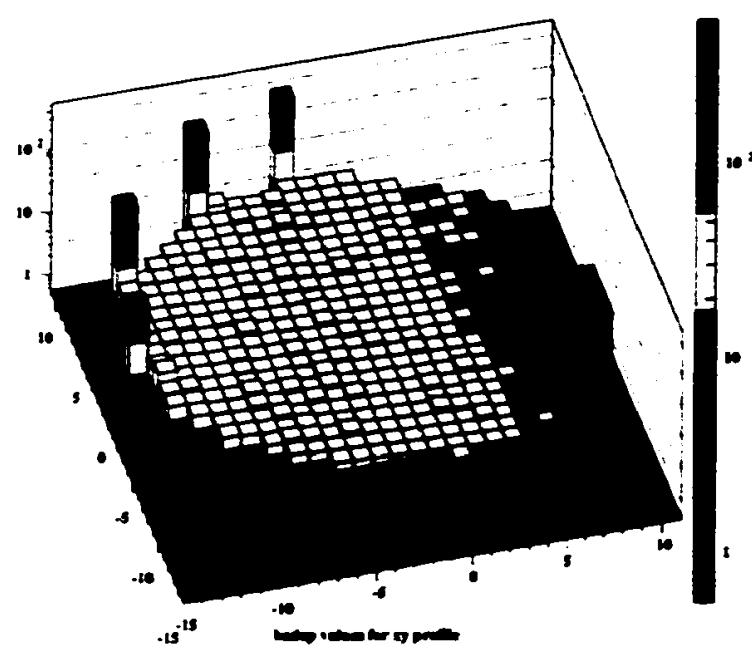

(a) Bad spill map

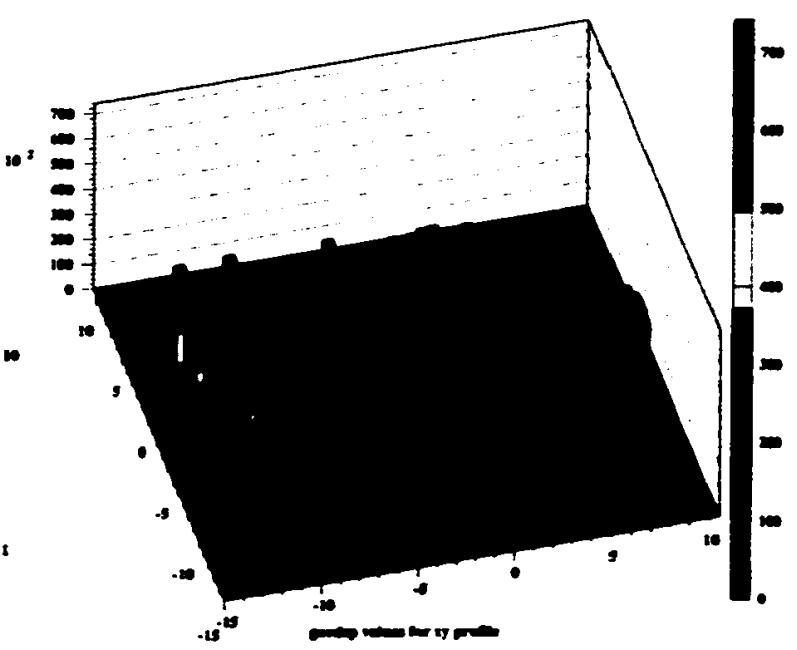

(b) Good spill map

Figure 2.6: "Spill maps" of average bad spill signal (a) and good spill signal (b) across the raster range for Run 3450. The spikes along the upper edge of the bad spill map indicate scraping is occurring for those raster positions. The good spill map is showing the raster pattern is generally centered on the target cell, although the edge of the target frame is showing up as the high signals on the low $X$ edge. The larger signals through the target center at $Y=0$ are due to the NMR coil.

an iron ring surrounding the beam line. A coil of wire is wrapped around the ring. When the beam pulse passes through the ring, it induces a magnetic field through the ring, which induces a current in the wire. The signal from the wire is amplified and measured in an ADC. Each toroid has a calibrator consisting of a wire passing through the ring. The calibration signal is provided by a monitored capacitor discharged through an attenuation circuit. The toroid control software sets both the attenuation factor and the amplification gain used by the toroid system. The toroid current measurement under E155 conditions is accurate to about $0.5 \%$, judging from the calibration response [59]. Another estimate of the accuracy of the toroid measurement is done by comparing the two toroids.

There are two "spill monitors" used in ESA, to monitor the beam quality and steering on a pulse-by-pulse basis. The "bad spill" monitor consisted of a 5.75 " by $10 "$ by $0.5 "$ scintillator paddle instrumented with a $2 "$ photomultiplier tube [60]. 
The bad spill monitor is located in the entrance alcove. about $50 \mathrm{~m}$ upstream of the target. A large bad spill signal indicates that the beam is scraping collimators in the beam pipe upstream of ESA, due to poor beam steering or low quality beam. The "good spill" monitor is located near the target position either slightly upstream or downstream of the target depending on the date within the experiment. This monitor was sensitive to secondary particles produced in beam interaction with the target. The good spill signal was proportional to the target thickness: therefore. it was used to check for target settling or beam misalignment. The two plots in Fig. 2.6 show the average good and bad spill monitor signals as functions of raster location. The isolated spikes along the high- $Y$. low- $X$ edge of the bad spill plot indicate the beam is slightly mis-steered as it enters ESA: if the bad spill plot showed more raster positions affected. the beam would be re-steered. The good spill plot is largely uniform, indicating that the raster pattern is centered on the target cell. The few channels with large good spill signal at low- $X$ may indicate the pattern is near the cell edge. The slight enhancement of the good spill signal along the $Y=0$ line is due to the NMR coil.

The experiment's primary measure of the beam position and size at the target is provided by the foil array. The foil array is located in the beam line downstream of the target. There are two arrays of 48 foil pairs. one array oriented horizontally; and one array oriented vertically. Each foil pair consists of the emitter and collector foils. with a high voltage potential maintained between them. When the beam passes through a foil, secondary electrons are produced from the emitter and gathered by the collector foil. producing a signal on the collector foil that is read out by an ADC [4]]. The beam spot size as measured by the foil array was about $1.0-1.2 \mathrm{~mm}$ horizontally, and $0.5-0.8 \mathrm{~mm}$ in the vertical direction. 


\subsubsection{Beam Polarization Measurement}

The beam polarization was measured using Møller polarimetry. The measured asymmetry in Møller scattering, in which polarized beam electrons elastically scatter from polarized atomic electrons, depends upon the beam and target electron polarizations, and the Møller asymmetries. which only depend on the center of mass scattering angle. For scattering with a center-of-mass angle near $90^{\circ}$, both electrons exit the target with half the incident energy. The asymmetry may be measured using either a single-arm polarimeter, in which only one electron is detected. or a doublearm polarimeter, in which both electrons are detected in coincidence. The E1.5.5 Møller polarimeter contained a set of double-arm detectors and a set of single-arm detectors [61].

The spin dependent cross-section for Møller scattering is given by.

$$
\frac{d \sigma}{d \Omega}=\frac{d \sigma_{0}}{d \Omega}\left(1+\sum_{i, j=x, y, z} P_{B}^{i} A_{i j}\left(\theta_{C . M}\right) P_{T}^{J}\right) .
$$

where $P_{B}^{i}$ and $P_{T}^{j}$ are the components of the beam and target polarizations, $d \sigma_{0} / d \Omega$ is the unpolarized cross-section, and $\boldsymbol{A}_{i j}\left(\theta_{C M}\right)$ is the asymmetry for the $i . j$ configuration of beam and target spins [61]. The $z$ axis is along the beam direction. and the $y$ axis is normal to the scattering plane [61]. In this scattering coordinate system. the asymmetries $A_{x y}, A_{y x} . A_{z y}$, and $A_{y z}$ are all zero: the asymmetries. $A_{x z}=A_{z x} \propto \sqrt{2 m /(E+m)}$, vanish at high values of the incident electron energy. The only contributing asymmetries are $A_{x x}, A_{y y}$ and $A_{z z}$. with

$$
\begin{aligned}
-A_{x x}=A_{y y} & =\frac{\sin ^{4} \theta_{C M}}{\left(3+\cos ^{2} \theta_{C M}\right)^{2}} . \\
A_{z z} & =-\frac{\left(\bar{\tau}+\cos ^{2} \theta_{C M}\right) \sin ^{2} \theta_{C M}}{\left(3+\cos ^{2} \theta_{C M}\right)^{2}},
\end{aligned}
$$

where $\theta_{C . M}$ is the center of mass scattering angle [62]. For longitudinal polarization of both the target and beam electrons, the cross-section becomes [62].

$$
\frac{d \sigma}{d \Omega}=\frac{d \sigma_{0}}{d \Omega}\left(1+P_{\bar{B}}^{z} A_{z z} P_{\bar{T}}^{z}\right)
$$


The measured asymmetry between Møller scattering rates for the electrons polarized parallel $(\uparrow \uparrow)$ and antiparallel $(\downarrow \uparrow)$ to the target electron polarization is related to $A_{z=}$ and the magnitude of the beam and target polarizations. $P_{B}$ and $P_{T}$. by:

$$
A_{\text {Moller }}=\frac{d \sigma^{\pi}-d \sigma^{\downarrow \uparrow}}{d \sigma^{\Uparrow \uparrow}+d \sigma^{\downarrow \uparrow}}=P_{B} A_{z z} P_{T}
$$

Then, for Møller scattering from a target of known polarization. the beam polarization is given by,

$$
P_{B}=\frac{A_{\text {Moller }}}{A_{z=} P_{T}}
$$

Just as with the deep inelastic scattering asymmetry, the Moller asymmetry is determined from the asymmetry of the scattering rates with corrections:

$$
A_{\text {Møller }}=\frac{N^{\downarrow} / Q^{\downarrow}-N^{\dagger} / Q^{\uparrow}}{N^{\downarrow} / Q^{\downarrow}+N^{\dagger} / Q^{\dagger}}
$$

The scattering angle and momentum in the laboratory are both related to the center of mass scattering angle and the center of mass energy. If the target electron is assumed to be a free particle at rest, then the square of the center of mass energy: $s_{0}$, is given by $[63]$.

$$
s_{0}=2 p_{b} m_{e}
$$

where $p_{b}$ is the beam momentum, and $m_{e}$ is the electron mass. The scattered electron has a laboratory momentum, $p^{\prime}$, defined by Eq. 2.16 and, for small angles. the laboratory scattering angle, $\theta$, is defined by Eq. 2.17 [63].

$$
\begin{gathered}
p^{\prime}=\frac{p_{b}}{2}\left(1+\cos \theta_{C M}\right) \\
\theta^{2}=\frac{1}{p_{b} p^{\prime}} \frac{s_{0}}{2}\left(1-\cos \theta_{C M}\right)=2 m_{e}\left(\frac{1}{p^{\prime}}-\frac{1}{p_{b}}\right)
\end{gathered}
$$

Equation 2.17 shows the correlation between $\theta$ and $p^{\prime}$, in the case of target electrons at rest. This correlation is used to separate the Møller elastic peak from the background. 
However, the target electrons are neither free nor at rest, but are bound in atomic shells with some net momentum. The Levchuk effect [64] is a smearing of the laboratory scattering angle due to the momentum of the bound target electrons. To leading order, the square of the center of mass energy is [6.3]

$$
s_{1}=s_{0}\left(1-\overrightarrow{p_{t}} \cdot \hat{n} / m_{e}\right)
$$

where $\overrightarrow{p_{t}}$ is the target electron momentum and $\hat{n}$ is the direction of motion of the beam electron. The scattered momentum dependence upon the center of mass angle does not change. so Eq. 2.16 is still valid. Equation 2.17 is modified by replacing $s_{0}$ with $s_{1}$, resulting in $[6: 3]$,

$$
\theta^{2}=\frac{1}{p_{b} p^{\prime}} \frac{s_{1}}{2}\left(1-\cos \theta_{C M}\right)=2 m_{e}\left(\frac{1}{p^{\prime}}-\frac{1}{p_{b}}\right)\left(1-\overrightarrow{p_{t}} \cdot \hat{n} / m_{e}\right) .
$$

The Levchuk effect results in a different amount of smearing of $\theta$ for scattering from different atomic shells, so if the detectors have a high $\theta$ resolution, the polarized electrons ( $\mathrm{M}$ - and $\mathrm{N}$-shells) and the unpolarized electrons ( $\mathrm{K}$ - and L-shells) will have different detected angular distributions resulting in a distortion of the measured asymmetry and beam polarization of up to $15 \%$ [6:3]. In the analysis. the polarized and background subtracted unpolarized distributions are integrated over the angular width of the smeared distributions to correct for the Levchuk effect [61].

\section{Moller Target and Analyzing Magnet}

In the E15.5 Møller polarimeter, the polarized target electrons are provided by severa! thin ferromagnetic foils, which may be individually moved into the beam path. The foils are made of Vacoflux, which is composed of $49 \%$ Co. $49 \% \mathrm{Fe}$. and $2 \% \mathrm{~V}$ by weight. The foils were about $3 \mathrm{~cm}$ wide, $35 \mathrm{~cm}$ long, and had thicknesses of either $20 \mu \mathrm{m}, 30 \mu \mathrm{m}, 40 \mu \mathrm{m}$, or $154 \mu \mathrm{m}$. The foils were oriented at an angle of $20.7^{\circ}$ to the horizontal $[61,65]$. A Helmholtz coil surrounded the target chamber 
and was used to polarize the target electrons. The Helmholtz coil provided a field of about 100G, which magnetized the foil to near saturation. By measuring the foil magnetization. the target electron polarization may be determined using.

$$
P_{T}=\frac{M}{n_{e} \mu_{B}} \frac{g^{\prime}-1}{g^{\prime}} \frac{g_{e}}{g_{e}-1}
$$

where $M$ is the bulk magnetization of the foil. $n_{e}$ is the electron density. $\mu_{B}$ is the Bohr magneton, $g_{e}$ is the free electron $g$ factor, and $g^{\prime}$ is the magneto-mechanical ratio for the Vacoflux alloy [61]. The resulting electron polarization is about \pm 0.08 along the beam direction [61], which corresponds to a net alignment of about 2.1 electrons per atom.

The scattered electrons passed through a mask with an aperture above and below the central aperture for the unscattered beam. The mask gives a $\theta$ acceptance of 3.59 to $8.96 \mathrm{mrad}$, with azimuthal $(\phi)$ acceptances of 0.20 rad for the upper aperture, and 0.22 rad for the lower aperture [61]. The mask permitted electrons with center-of-mass scattering angles between about $75^{\circ}$ and $105^{\circ}$ to enter the detectors $[65]$.

The Møller analyzing magnet is a dipole magnet oriented to bend the electron trajectories horizontally. The unscattered beam passes through an iron septum which shields it from the field of the magnet. The magnet provides an integrated field. $\int B d l \sim 3.8 \mathrm{Tm}[66]$, with a central field of about $1 \mathrm{~T}[4 \bar{\tau}]$. Since the scattering angle and momentum are inversely related by Eq. 2.19, it is the low-momentum electrons at large scattering angles that are most deflected by the magnet. The Møller electrons form a stripe at the detectors, as shown in Fig. 2.. .

\section{Detectors}

The detector systems for the Møller polarimeter consisted of five silicon detectors which form the upper and lower single arm detectors, and two arrays of seven 


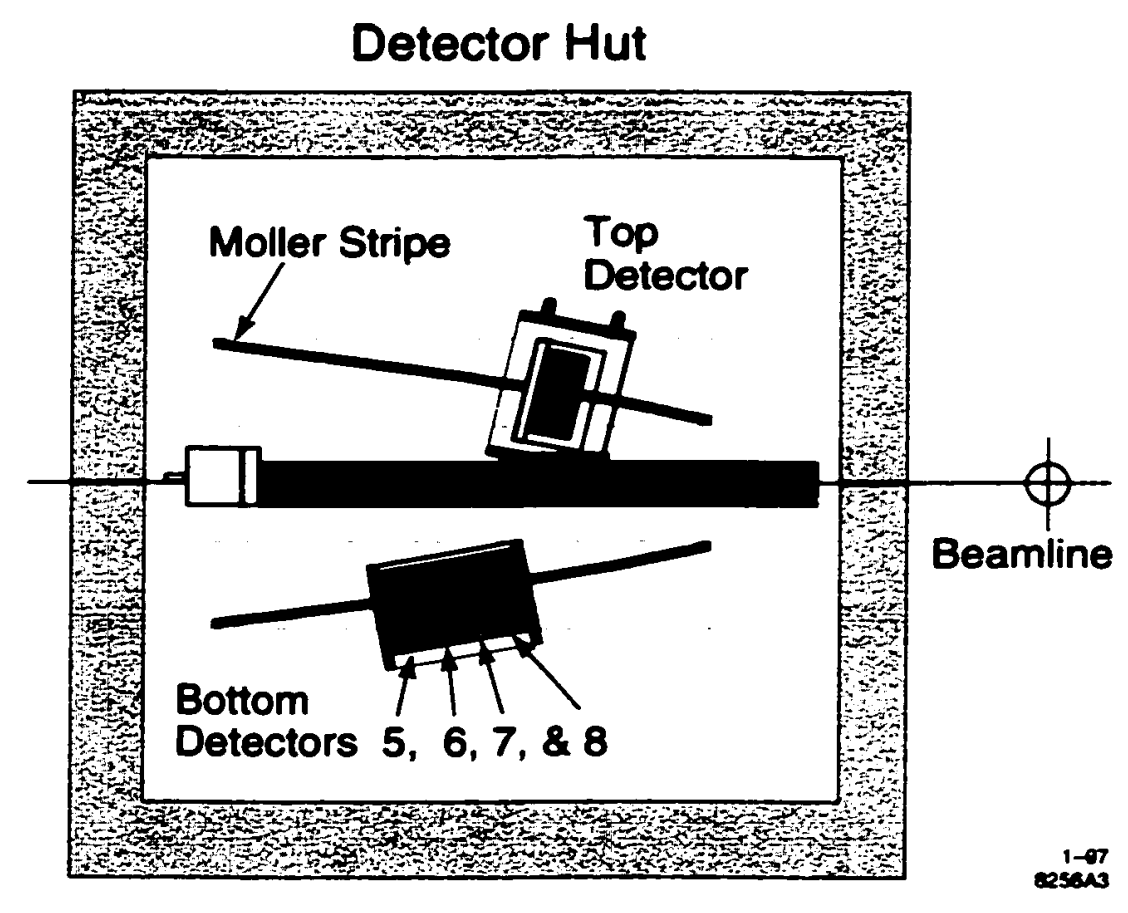

Figure 2.7: The E154 Møller polarimeter detector package [61]. In the Møller detector systems used in E155, the top and bottom detectors have been switched in location (Detectors 5-8 occupy the top position in E155; the fine detector, shown here in the top position, occupied the position of Detectors 5-8 in this figure. The double arm detectors are not shown in this figure, but were approximately positioned within the dashed rectangles aligned along the Møller stripe.

SF-6 lead glass blocks which form the double arm detectors. The lead glass blocks are $10 \times 10 \mathrm{~cm}$ with a depth of $30 \mathrm{~cm}[67]$. The lead glass blocks are located along the Møller stripe, behind the silicon detectors [67]. Each silicon detector contained two $4 \mathrm{~cm} \times 6 \mathrm{~cm}$ silicon pad devices oriented to form a detector $4 \mathrm{~cm}$ wide and $12 \mathrm{~cm}$ tall.

The lower single arm detector consisted of one finely segmented silicon detector, and the upper detector was a set of four coarse segmented silicon detectors [68]. The configuration of the silicon detectors was similar to that used in the E154 polarimeter, with the exception that the coarse and fine detectors were respectively in the lower and upper positions in E154 [61]. The fine detector had 48 instrumented channels along the total height of $12 \mathrm{~cm}$, with each channel having a width of $2.18 \mathrm{~mm}$ in the vertical direction. The detector was rotated so the channels were 
parallel to the Møller stripe. The fine detector was mounted on a remotely controlled $X-Y$ stage, and could be positioned anywhere within the acceptance [61]. The four coarse detectors (Detectors 5-8) each had twelve instrumented channels along the total height of $12 \mathrm{~cm}$ : each channel was $8.69 \mathrm{~mm}$ wide. They were positioned side by side in the Møller acceptance and were rotated so the channels were parallel to the Møller stripe as shown in Fig. 2.7 [61]. The total charge deposited in each of the 96 detector channels was integrated over the spill length by charge sensitive preamplifiers [61]. The preamplifier output was digitized by SLAC-designed ADCs located in the beam CAMAC crate in the counting house. These ADCs were only read out during Møller runs.

\section{$\underline{\text { Møller Polarimeter Data and Beam Polarization Results }}$}

Because the Møller polarization measurement requires inserting the Møller target into the beam, this measurement could not take place during normal data collection. During the Møller runs, the beam focus in ESA was altered slightly, the Møller target was inserted. and the Møller magnet B0 was activated. Møller data were collected at beam currents from $1 \times 10^{9}$ to $6 \times 10^{9}$ electrons/spill. Both single arm and double arm Møller polarization measurements were made. using foils of several different thicknesses.

The overall results were $P_{b}=0.813 \pm 0.020$ from the single arm polarimeter. and $P_{b}=0.805 \pm 0.032$ from the double arm polarimeter [69]. The errors in these results are dominantly systematic, since the statistical error from each measurement was about 0.002 , and the two results above include many measurements made throughout the experimental run. The beam polarization used in the analysis was the weighted average of the two results, $P_{b}=0.810 \pm 0.020$. The error is taken to be 0.02 due to the correlation between the errors [4i]. 

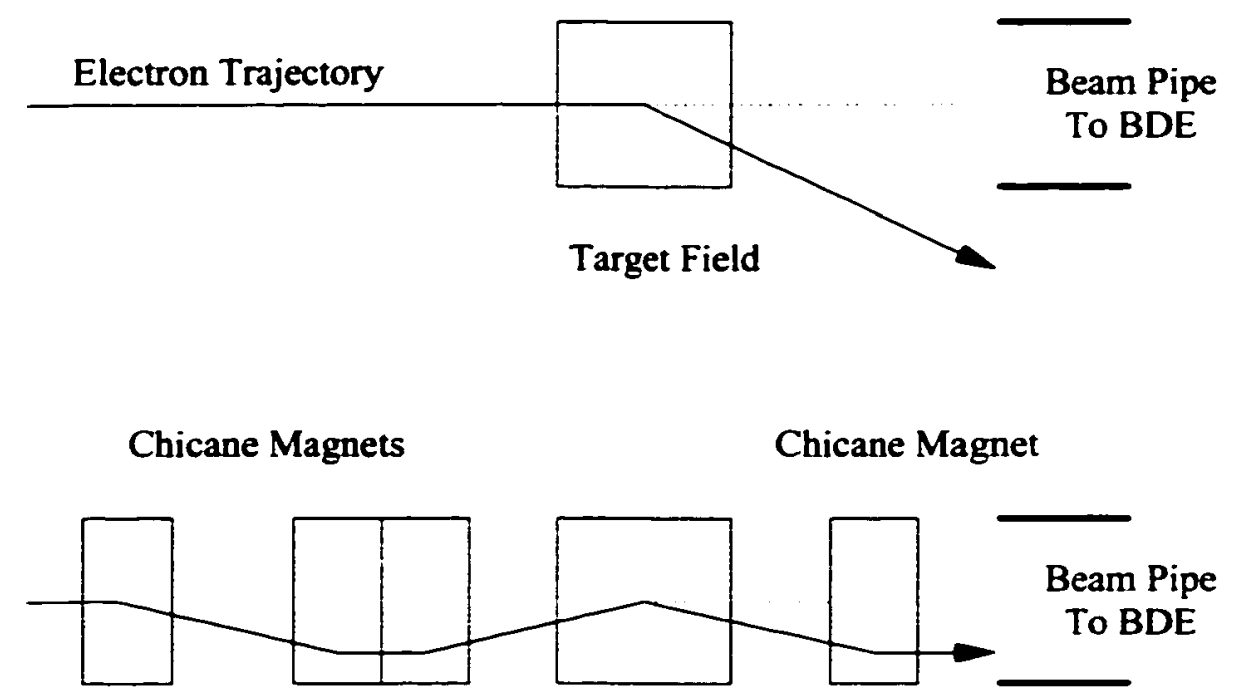

Figure 2.8: Rough schematic of the chicane system. Upper drawing shows the beam trajectory in transverse mode without the chicanes; lower drawing shows the beam trajectory with the chicanes.

\subsubsection{ESA Chicane Magnet System}

During the measurement of the longitudinal asymmetry the unscattered beam passes undeflected through the target magnetic field. and is transmitted to Beam Dump East (BDE). During the transverse asymmetry measurement. the target magnetic field is oriented perpendicular to the beam direction. so the unscattered beam is deflected from the path to the beam dump. as is shown in the upper block diagram of Fig. 2.8. Since this is undesirable for both the accelerator operations and experimental backgrounds, additional magnets are used to correct the trajectory of the unscattered beam so that it is transmitted through ESA into BDE.

This chicane bend trajectory is produced using four dipole magnets. three located before the target magnet, and one located after the target magnet. A schematic of the beam trajectory through the chicane and target magnets is shown in the lower block diagram of Fig. 2.8. The first chicane magnet deflects the beam in the same direction as the target magnet, while the other three magnets deflect the beam in the opposite direction as the target magnet. All four of the chicane magnets are operated at the same $\int B d l$, which is chosen to be half of the $\int B d l$ of 
the target magnet. The beam bend angle produced by each chicane is then half of the bend angle produced by the target magnet. The positions of each of the four magnets is chosen so that the beam trajectory passes through the center of the target, as is sketched in the lower block drawing of Fig. 2.8. Additionally. the chicane system precesses the momentum and spin vectors to correct for the precession in the target magnetic field, so that both the momentum and spin vectors are parallel with the momentum and spin vectors of the beam before entering the chicane and target system.

\subsection{Polarized Nuclear Target}

To achieve the polarization of target nuclei desired for E15.5, the process of Dynamic Nuclear Polarization (DNP) is used to align the target nuclei parallel or antiparallel to a $5 \mathrm{~T}$ magnetic field at a temperature of $1 \mathrm{~K}$. The polarizations of the target nuclei and the other polarizable nuclear species in the polarized target cells were measured using nuclear magnetic resonance (N.MR).

E15.5 used two types of polarized target material: frozen ammonia $\left({ }^{15} \mathrm{NH}_{3}\right)$ for the proton target, and lithium deuteride $\left({ }^{6} \mathrm{Li}^{2} \mathrm{H}\right.$ or $\left.{ }^{6} \mathrm{LiD}\right)$ for the deuteron target. The reason the isotopes ${ }^{15} \mathrm{~N}$ and ${ }^{6} \mathrm{Li}$ are used instead of the more common isotopes ${ }^{14} \mathrm{~A}$ and ${ }^{7} \mathrm{Li}$ is that the nuclear corrections for ${ }^{15} \mathrm{~N}$ and ${ }^{6} \mathrm{Li}$ are simpler than for ${ }^{14} \mathrm{~N}^{\circ}$ and ${ }^{\top} \mathrm{Li}$. This can be seen using a very simple picture of the nuclei, although a more careful discussion of the nuclear physics involved in the target will be presented in Section 5.6.1. When ${ }^{15} \mathrm{~N}$, with 7 protons and 8 neutrons. is polarized. the unpaired proton will polarize but the neutrons, being all paired. will not. ${ }^{14} \mathrm{~N}$ has an unpaired proton and an unpaired neutron. both of which will polarize. Therefore, ${ }^{15} \mathrm{~N}$ in a polarized target acts like a proton (actually the spin of the unpaired proton in ${ }^{15} \mathrm{~N}$ is antiparallel to the ${ }^{15} \mathrm{~N}$ spin, so it acts like a negatively polarized proton), whereas ${ }^{14} \mathrm{~N}$ behaves like a proton and neutron. ${ }^{6} \mathrm{Li}$ has three protons and three neutrons, so 


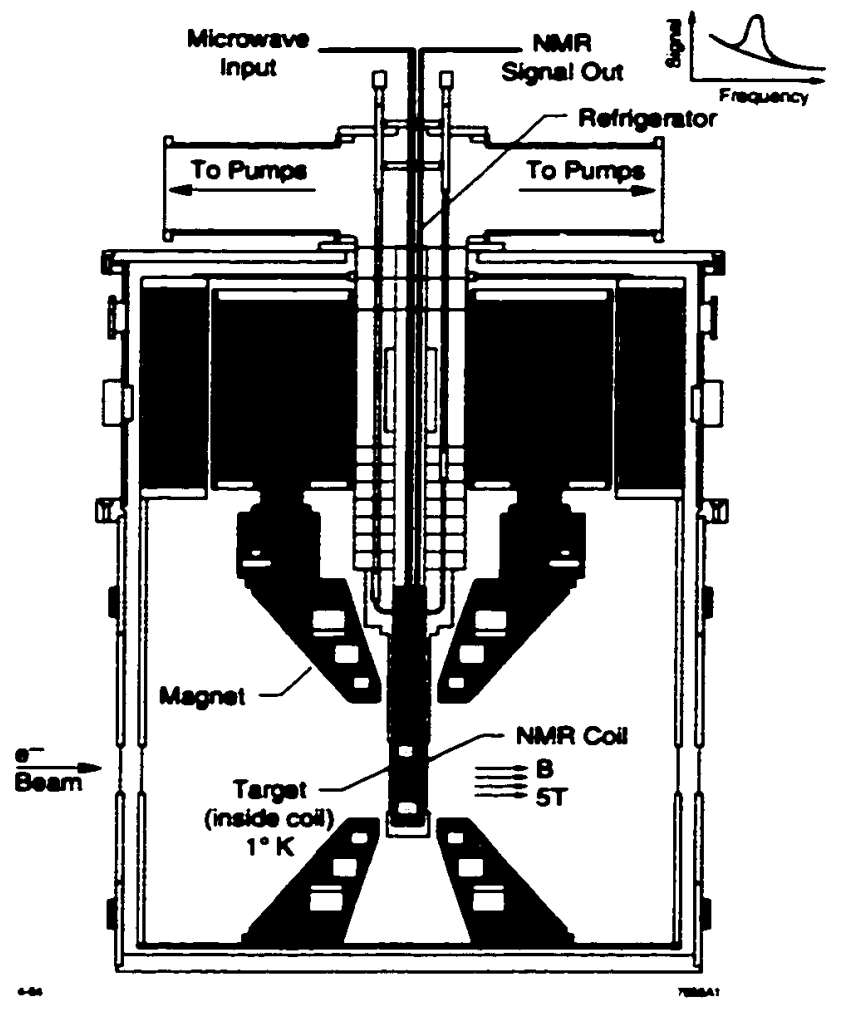

Figure 2.9: Schematic cross-section of the target assembly.

both a proton and neutron would polarize. However, the structure of ${ }^{6} \mathrm{Li}$ acts like a bound state of ${ }^{4} \mathrm{He}$ and ${ }^{2} \mathrm{H}$, so when using ${ }^{6} \mathrm{LiD}$ half of the nucleons behave like deuterons (approximately). ${ }^{\top} \mathrm{Li}$ has three protons and four neutrons and presents a polarized proton. The proton target had small contaminations of ${ }^{14} \mathrm{~N}$ and ${ }^{2} \mathrm{H}$, while the deuteron target had small contaminations of ${ }^{7} \mathrm{Li}$ and ${ }^{1} \mathrm{H}$. The nuclear corrections for all of the polarizable species in the target materials will be discussed in Section 5.6 .1 .

\subsubsection{Target Assembly}

The 5 Tesla super-conducting Helmholtz coil magnet defines the polarization axis, and is required to split the energy level degeneracies needed by DNP. The target magnet may be aligned with the field either parallel to the electron beam direction, as pictured in Fig. 2.9, or perpendicular to the electron beam direction. The 
realignment of the magnetic field is done by rotating the target magnet assembly. When the target field is aligned perpendicular to the beam direction. the chicane magnets (see Section 2.2.3) are required for control of the unscattered beam. The magnetic field is uniform to one part in $10^{4}$ over a $3 \mathrm{~cm}$ diameter about the coil center [i0]. The ${ }^{4}$ He evaporation refrigerator is located in the center of the magnet. This refrigerator provides about $1.5 \mathrm{~W}$ of cooling power at around $1 \mathrm{~K}[\bar{\tau} 1]$.

\subsubsection{Target Insert}

The target insert fits in the central bore of the refrigerator. and can be moved vertically within the refrigerator, positioning any of several different target cells in the beam. Each target insert contains two polarized target cells. a large unpolarized solid target ("dummy" target). a small unpolarized solid target. and a hole target. In addition to providing the ability to move between target positions. the target insert carries the services, such as the NMR cables, microwave waveguide, heater wires, etc., down to the target cells at the nose of the refrigerator.

The polarized target cells are cylinders about $3 \mathrm{~cm}$ long with an inner diameter of $2.636 \mathrm{~cm}$ [72]. They are made of copper-plated aiuminum-6061 cavities. with aluminum-1145 windows [ 72$]$. Each cell has an independent wave guide to deliver the microwave power needed for the DNP process. Each cell contains at least one set of NMR coils, to permit NMR measurements of the species of primary and secondary interest. The target material is in the form of small grains. produced by crushing the frozen material, then sifting it to choose the grain size. The grain size used for the $\mathrm{NH}_{3}$ was most often 2.5-3.5 mm, and the LiD grain size was most often 1.0-2.0 mm [72]. After the material was crushed and sifted, it was irradiated with an intense low energy electron beam to produce the paramagnetic radicals required by the DNP process. The $30 \mathrm{MeV}$ electron beam at the SUNSHINE facility at

Stanford Liniversity was used to deliver an electron flux of $1.3-4.5 \times 10^{17} \mathrm{e}^{-} / \mathrm{cm}^{2}$ to 
the LiD [73]. The $\mathrm{NH}_{3}$ received pre-irradiation doses of $1-4 \times 10^{17} \mathrm{e}^{-} / \mathrm{cm}^{2}$ at similar electron energies $[71,72]$. Generally, the two target cells on an insert were filled with the same material. due to differences in the material lifetime and the anneal process between the $\mathrm{NH}_{3}$ and LiD.

The two unpolarized solid targets are included to provide a way of determining the mass-thickness of the polarized targets from experimental data. The solid target material and thickness was chosen to be a close match to the polarized target material. The dummy targets and the small targets are $0.271^{*}$ thick disks. with diameters of $1.068^{\prime \prime}$ and $0.394^{\prime \prime}$, respectively [72]. Pyrolytic graphite was chosen to match the $\mathrm{NH}_{3}$, as a $0.270^{\circ}$ thick disk with a mass-thickness of $1.51 \mathrm{~g} / \mathrm{cm}^{2}$ and 0.035 .3 radiation lengths (r.l.), a close match to the $1.6 .5 \mathrm{gm} / \mathrm{cm}^{2}$ and $0.0381 \mathrm{r.l}$. thickness of the $\mathrm{NH}_{3}[74]$. Beryllium $\left({ }^{9} \mathrm{Be}\right)$. with thicknesses of $1.27 \mathrm{gm} / \mathrm{cm}^{2}$ and $0.0194 \mathrm{r} .1$., was chosen to match the LiD, which has a thickness of $1.48 \mathrm{gm} / \mathrm{cm}^{2}$ and 0.0193 r.l. [74]. The "dummy" target consists of a cavity identical to the polarized target cells with one of the large solid targets inserted at the upstream side [ 72$]$. The beam may be rastered upon the dummy target. exactly as for the polarized targets. When the thickness of the liquid helium is included. the total thickness of the dummy targets is $1.85 \mathrm{gm} / \mathrm{cm}^{2}\left(0.0389 \mathrm{r.l}\right.$.) for the graphite and $1.61 \mathrm{gm} / \mathrm{cm}^{2}$ $(0.0230$ r.l.) for the beryllium [74]. Since the small targets are so much smaller than the target cells, they cannot be rastered upon. The small target is used to have a cross-check of scattering rates from the different target materials. The small target is the alternate material from the dummy target. On a LiD-filled target insert. the dummy target would be beryllium, and the small target would be graphite, whereas an $\mathrm{NH}_{3}$ insert would have graphite and beryllium as the dummy and small targets. respectively. The hole target is a hole in the aluminum support of the target insert. This target position allows measurements of scattering associated with the vacuum windows and the liquid helium in which the insert is immersed. 


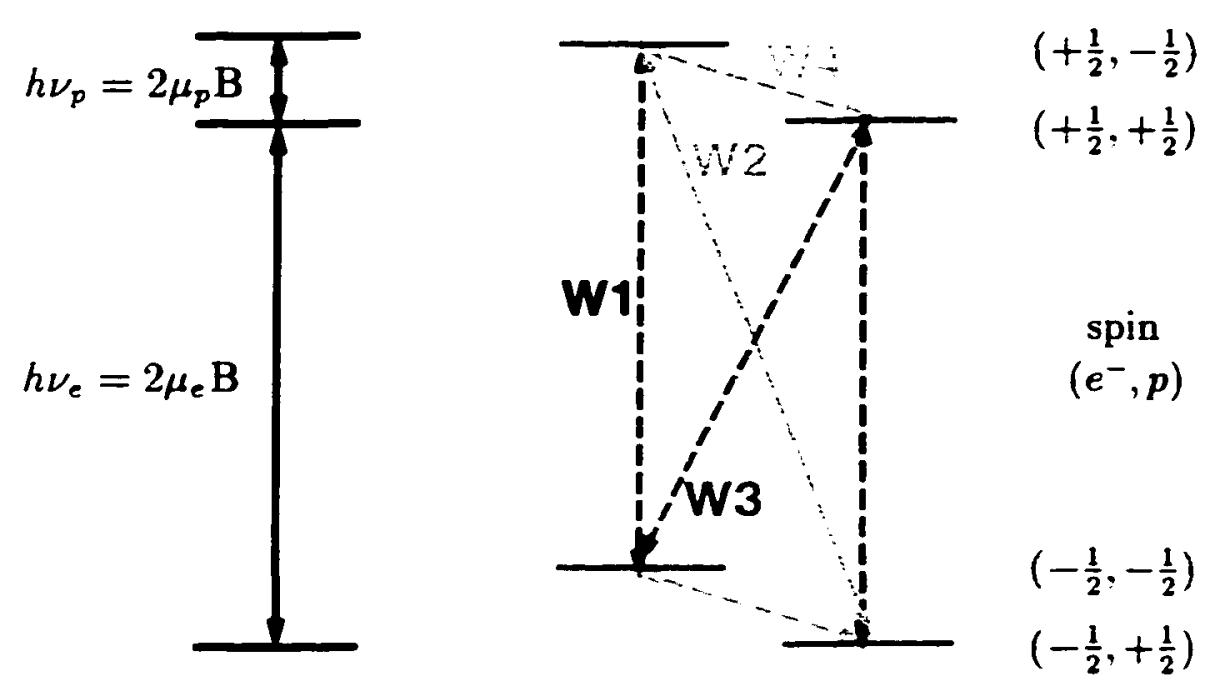

Figure 2.10: Energy level schematic for the electron-proton system in an external magnetic field. By driving either the W3 or W2 transitions, the proton will become polarized in the positive or negative direction.

\subsubsection{Dynamic Nuclear Polarization}

The process of dynamic nuclear polarization (DNP) uses microwave radiation to polarize the nuclei of a paramagnetic material. In a high magnetic field and low temperature environment, the electrons of a paramagnetic material will have a net spin polarization antiparallel to the magnetic field due to splitting of the degeneracy between the electron spin states. Application of microwaves of the correct frequency (transitions W2 or W3 shown in Fig. 2.10) will cause a transfer of the electron polarization to the nucleus. In the case of the E155 target, the bulk material is not paramagnetic, but paramagnetic centers are produced in the material by irradiation with an intense electron beam. The polarized nuclei of the isolated paramagnetic centers are able to transfer their polarization to adjacent nuclei, thus polarizing the other material as well.

In a magnetic field, the atomic energy levels of an unpaired electron have energy splitting due to the Zeeman effect (coupling between the electron spin and the magnetic field) and coupling between the nuclear spin and the magnetic field. Fig. 
2.10 diagrams the energy levels. and the transitions between the energy levels for a hydrogen atom in a magnetic field of strength. B. In a $5 \mathrm{~T}$ magnetic field. the microwave frequency corresponding to the electron spin-flip (transition $W_{1}$ ) is $\nu_{e} \sim$ $140 \mathrm{GHz}$, and the frequency of the proton spin-flip transition (transition $\mathrm{W} 4$ ) is about $\nu_{p} \sim 213 \mathrm{MHz}$. The transitions $\mathbf{W} 2$ and $\mathrm{W} 3$, in which both the electron and proton switch spin orientations, are in principle forbidden by dipole selection rules. but may proceed slowly (the rate is about $10^{-3}$ that of the $W_{1}$ transition) due to the hyperfine interaction between the electron and nuclear spins [75].

By applying microwave radiation at the frequency to drive either the transition WV2 (microwave frequency $\nu_{e}+\nu_{p}$ ) or W3 (microwave frequency $\nu_{e}-\nu_{p}$ ). the electronnucleon system is excited into a anti-aligned electron spin state. from which the dominant de-excitation is via transition $W 1$. The $W 1$ transitions are relatively rapid. whereas the W4 transitions between states of opposite nuclear polarization are relatively long. In the E15.5 target. about 1 Watt of microwave power is delivered to the target material at either the $W 3$ transition frequency. which generates a positive enhanced polarization (spins aligned to the field). or the $\mathrm{W} 2$ transition frequency. which generates a negatively enhanced polarization (spins anti-aligned to the field).

\subsubsection{Measurement of the Target Polarization}

The polarization of the target nuclei is measured using nuclear magnetic resonance. A material with a nuclear spin of $J$ will exhibit an energy level splitting into $2 J+1$ levels in a magnetic field, distinguished by the spin projection of the nuclear spin along the magnetic field. The energy difference between the sublevels will be given by,

$$
\Delta E=h \nu_{L}=g \mu_{N} B_{0}
$$

where $\nu_{L}$ is the Larmor frequency, $g=\mu / J$ is the $g$-factor of the nucleus with magnetic moment $\mu, \mu_{N}=3.152 \times 10^{-14} \mathrm{MeV} / \mathrm{T}$ is the nuclear magneton [4]. and 
$B_{0}$ is the magnetic field strength at the nucleus. A proton $(g=5.586)$ in a $5 \mathrm{~T}$ field would have a Larmor frequency of about $213 \mathrm{MHz}$. and a deuteron $(g=0.857)$ in that same field has a Larmor frequency of about $32.6 \mathrm{MHz}$. When radio frequency energy at the Larmor frequency irradiates the material, transitions between adjacent nucleon spin substates will occur, both energy-absorbing upward transitions and energy-emitting downward transitions. The susceptibility of the material. $\gamma$. is a complex function of the applied frequency. where we now switch notation to use angular frequency. $\omega=2 \pi \nu$,

$$
x(\omega)=x^{\prime}(\omega)+\imath x^{\prime \prime}(\omega)
$$

where $x^{\prime}$ and $x^{\prime \prime}$ are respectively the real and imaginary components of $\chi$. The polarization. $P$. of the nuclei in the sample is related to the susceptibility by.

$$
P=K \int_{0}^{\infty} x^{\prime \prime}(\omega) \mathrm{d} \omega
$$

where the constant $K$ depends upon the properties of the nucleus [76]. If the imaginary part of the material susceptibility can be measured over a sufficiently large range, then the polarization can be determined.

If a coil of inductance $L_{C}$ and resistance $r_{C}$ is embedded in the material. the complex susceptibility of the material will modify the impedance of the coil:

$$
Z_{C}(\omega)=r_{C}+\omega L_{C}(1+4 \pi \eta x(\omega))
$$

where $\eta$ is the effective filling factor of the coil [71]. Lising Eq. 2.22. the coil impedance becomes,

$$
Z_{C}(\omega)=r_{C}-\omega L_{C} 4 \pi \eta \chi^{\prime \prime}(\omega)+\imath \omega L_{C}\left(1+4 \pi \eta \chi^{\prime}\left(\omega^{\prime}\right)\right)
$$

The NMR coil is connected in series with an external resistor and variable capacitor. contained in a package known as a Q-meter [76], thus forming an LRC circuit [75]. 
When a radio frequency voltage, with some frequency $\omega$. at a constant current is applied to the circuit. the real part of the output voltage depends upon $V^{\prime \prime}(\nu)$, and thus the polarization. By sweeping the frequency of the radio frequency source around the Larmor frequency and measuring the voltage gain due to the material, the integral of $\chi^{\prime \prime}(\omega)$ about the resonance may be determined.

The background subtraction and calibration of the NMR signal requires knowledge about the material, coil, and Q-meter. However, this knowledge may be acquired through specific measurements using the NMR system, rather than being acquired a priori. The frequency dependence of the Q-meter signal (or Q-curve) can be determined by sweeping the radio frequency after moving the nuclei off resonance by shifting the magnetic field by $1 \%[70]$. The material then does not contribute to the NMR signal $(\chi(\omega) \approx 0)$, so the response of the Q-meter and coil is measured. This baseline is subtracted from all subsequent measurements using that Q-meter. The polarization calibration is done by finding the area under a baseline subtracted V.MR signal at a known polarization. This was done by measuring the NMR signal at thermal equilibrium.

A system of nuclear spins in thermal equilibrium in an external magnetic field will have populations in each spin orientation state described by the Boltzmann distribution.

$$
N_{i}=N \frac{\mathrm{e}^{-E_{i} / k_{B} T}}{\sum_{i} \mathrm{e}^{-E_{1} / k_{B} T}} .
$$

The thermal equilibrium polarization is the average spin projection divided by the total spin:

$$
\begin{aligned}
& P_{T E}=\frac{<m_{J}>}{J}=\frac{1}{N J} \sum_{i} m_{i} N_{i}=\frac{1}{J} \frac{\sum_{i} m_{i} \mathrm{e}^{-E_{i} / k_{B} T}}{\sum_{i} \mathrm{e}^{-E_{1} / k_{B} T}} \\
& P_{T E}=\frac{1}{J} \frac{\sum_{i} m_{i} \mathrm{e}^{g m_{1} \mu_{N} B / k_{B} T}}{\sum_{i} \mathrm{e}^{g m_{\mathrm{i}} \mu_{N} B / k_{B} T}} .
\end{aligned}
$$


For spin- $\frac{1}{2}$ nuclei. Eq. 2.28 becomes

$$
\begin{aligned}
P_{T E} & =2 \frac{\frac{1}{\frac{1}{2} \mathrm{e}^{\frac{1}{2} g \mu_{N} B / k_{B} T}}-\frac{1}{2} \mathrm{e}^{-\frac{1}{2} g \mu_{N} B / k_{B} T}}{\mathrm{e}^{-\frac{1}{2} g \mu_{N} B / k_{B} T}+\mathrm{e}^{-\frac{1}{2} g \mu_{N} B / k_{B} T}}=\frac{\mathrm{e}^{\mu B / k_{B} T}-\mathrm{e}^{-\mu B / k_{B} T}}{\mathrm{e}^{\mu B / k_{B} T}+\mathrm{e}^{-\mu B / k_{B} T}} \\
& =\tanh \left(\frac{\mu B}{k_{B} T}\right)
\end{aligned}
$$

where $\mu=\frac{1}{2} g \mu_{N}$ is the magnetic moment of the nucleus.

By making NMR measurements of the polarization at thermal equilibrium. the calibration of the NMR signal may be found. The enhanced polarization is given by:

$$
P_{e n h}=\frac{P_{T E}}{A_{T E}} \cdot A_{e n h}
$$

where $P_{T E}$ is the thermal equilibrium polarization. $A_{T E}$ is the area under the baseline-subtracted NMR signal for the thermal equilibrium (TE) measurement. and $A_{\epsilon n h}$ is the area under the baseline-subtracted NMR signal for the polarized target.

The polarizations of the other species in the target other than the species of interest are also needed. The polarizations of ${ }^{15} \mathrm{~N},{ }^{6} \mathrm{Li},{ }^{14} \mathrm{~N},{ }^{7} \mathrm{Li},{ }^{2} \mathrm{H}$ and ${ }^{1} \mathrm{H}$ can each be measured by using Q-meters with properly tuned resonant frequencies. However. the polarizations of the secondary species may instead be estimated from the polarization of the primary species using the theory of Equal Spin Temperature (EST) $[70]$.

\subsection{Particle Detection and Identification: Spectrometers}

When the beam interacts with the target, some of the incident electrons undergo deep inelastic scattering. The scattered electron's energy and scattering angle depend upon the kinematics of the DIS process, so measurement of the distribution of scattered electrons yields information about the interaction. However. scattered electrons are not the only type of particles produced by the interaction of the beam 


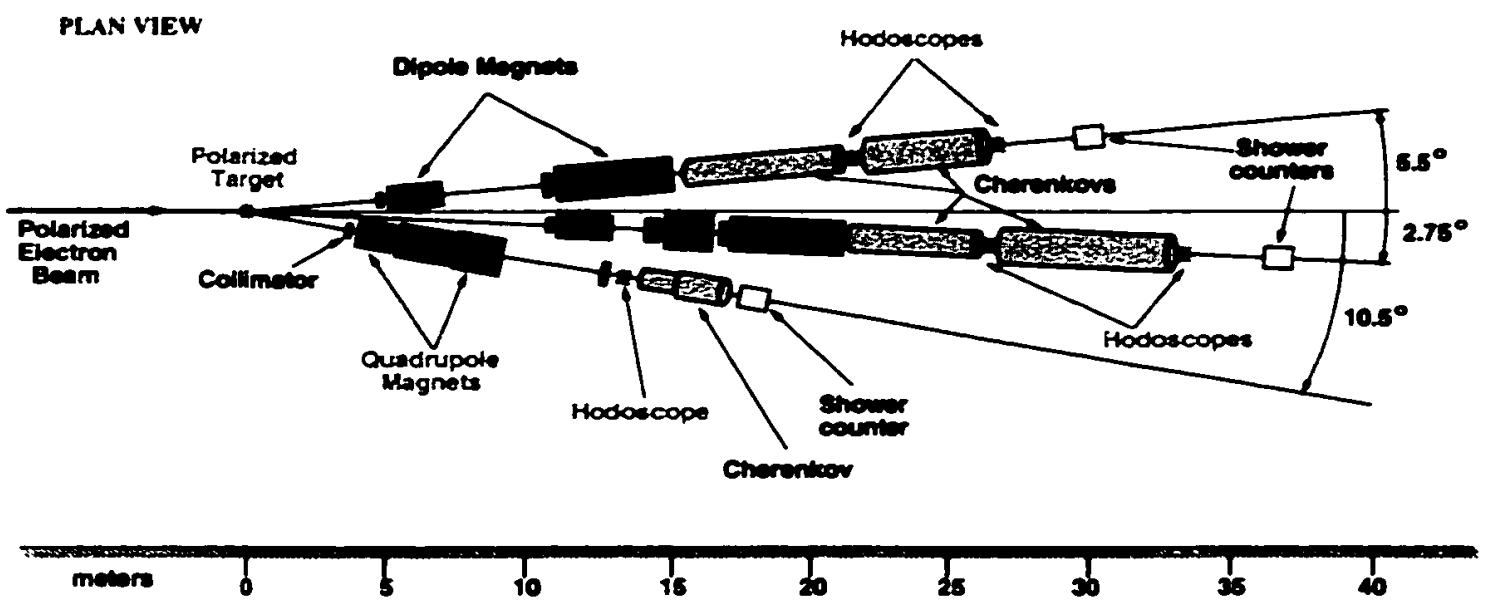

Figure 2.11: Schematic of the E155 spectrometer layout, showing the detector packages.

with the target. A method of particle identification is necessary so that only the deep inelastically scattered electrons are counted towards the physics asymmetry. Since this is an inclusive measurement, detection of the fragmentation products is not a goal.

Magnetic spectrometers containing hodoscopes are used to determine the momentum and angle of the particles emerging from the target. Threshold Cherenkov detectors partially distinguish between electrons and pions, forming one of the tools used in particle identification. Comparing the energy deposited in an electromagnetic shower calorimeter to the particle momentum provides another particle identification tool. A high energy electron will have a ratio of energy to momentum near one, since $E^{2}=P^{2}+m_{e}^{2} \sim P^{2}$ for $E>m_{e}$; but the deposited energy to momentum ratio for pions will be much lower, since pions generally don't deposit all of their energy in the electromagnetic calorimeter.

E155 used three spectrometers at central scattering angles of $2.75^{\circ}, 5.5^{\circ}$, and $10.5^{\circ}$. The $2.75^{\circ}$ and $5.5^{\circ}$ spectrometers were used previously by SLAC experiment E154 [23, 77]. The $10.5^{\circ}$ spectrometer was constructed for E155 [78, 79]. The kinematic coverage of each spectrometer is shown in Fig. 2.12. The three spectrometers each have a magnetic optics system and a system of hodoscopes which together pro- 


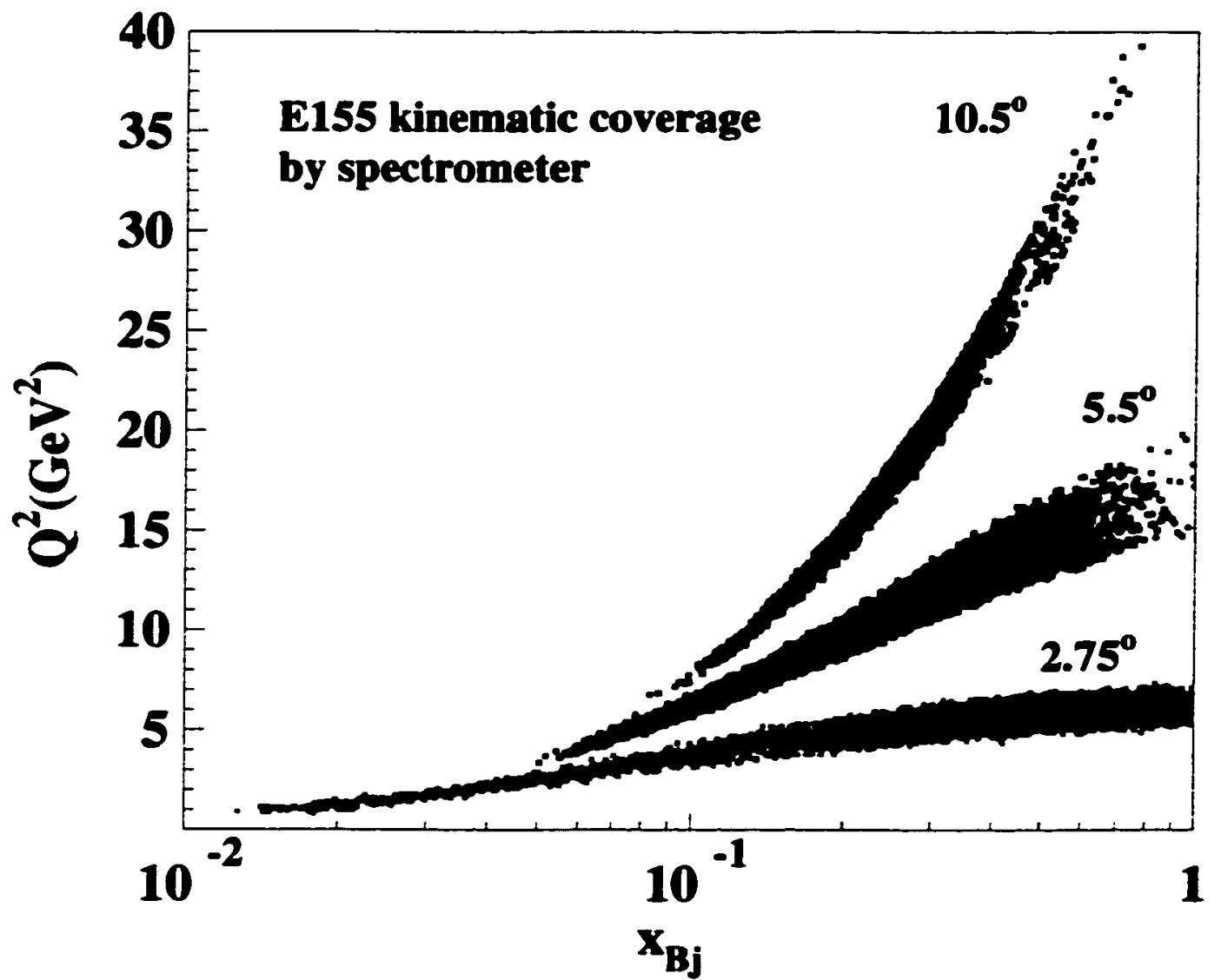

Figure 2.12: E155 kinematic coverage per spectrometer at a beam energy of $48.3 \mathrm{GeV}$; notice that the 10.5 degree spectrometer reaches a $Q^{2}$ nearly double the maximum in the $5.5^{\circ}$ spectrometer.

vide momentum and scattering angle reconstruction, threshold Cherenkov detectors that provide particle identification, and an electromagnetic shower calorimeter that measures the particle energy. Fig. 2.11 shows the layout of the three spectrometers with respect to the target and electron beam. The components of the $2.75^{\circ}$ and $5.5^{\circ}$ spectrometers will be discussed through the remainder of this section. The $10.5^{\circ}$ spectrometer will be discussed in Chapter 3 .

\subsubsection{Magnetic Optics \& Collimation}

Since a charged particle traveling through a magnetic field follows a curved trajectory which depends upon the momentum, a careful selection of magnets and collimators will permit particles with a certain momentum range to pass through 
DIEvatron (nes),

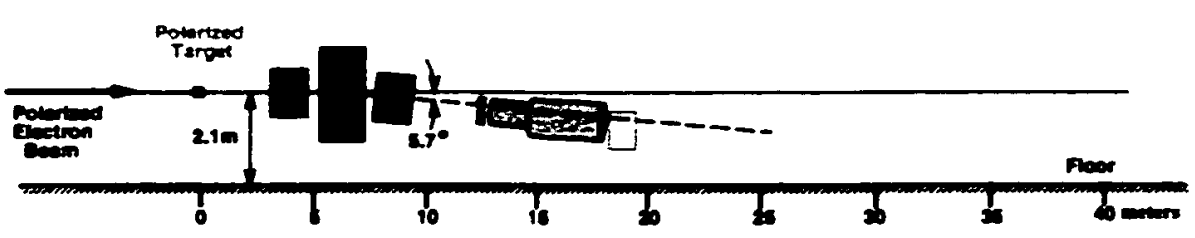

EEVATMOA (5.5*)

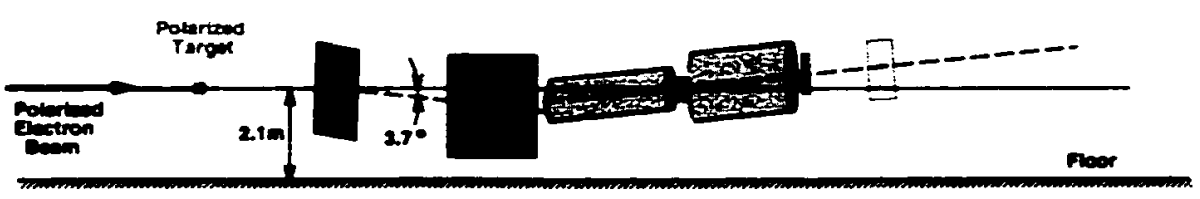

EIEVATION $1273^{\circ}$,

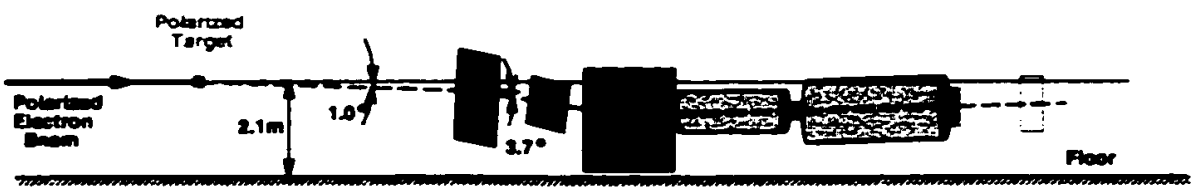

Figure 2.13: Side layout of the E155 spectrometers, showing the "S-bend" magnetic optics configuration in the $2.75^{\circ}$ and $5.5^{\circ}$ spectrometers.

the spectrometer optics. Collimators placed within the magnet system will stop particles whose momenta are not within the acceptable range. The magnetic optics may also be used to disperse the trajectories of particles with different momenta.

After the particle exits the magnetic optics, it will have a straight trajectory. If the magnetic fields in the spectrometer system are known, a measurement of the location and angle of the trajectory in the spectrometer may be used to "track" the particle through the magnetic optics system to determine the initial scattering angle and energy of the particle.

The $2.75^{\circ}$ and $5.5^{\circ}$ spectrometers use an "S-bend" magnetic optics configuration, shown in Fig. 2.13, which reduces the neutral particle background in the spectrometers by requiring neutral particles to have bounced twice off material along the path in order to enter the detector hut. The "S-bend" configuration uses two dipole magnets with opposite polarity to bend the trajectories first in one direction, then in the opposite direction. Using the "S-bend" or reverse bend configuration 
increases the solid angle acceptance as compared to two magnets bending in the same direction [80], due to a refocusing of the different momenta particles. The $2.75^{\circ}$ spectrometer has a quadrupole magnet located between the two dipole magnets. The quadrupole magnet defocuses the trajectories in the horizontal direction. to spread the instantaneous particle rate over the detector elements. The instantaneous particle rate in the $5.5^{\circ}$ spectrometer was not high enough to require a defocusing quadrupole in the magnetic system.

\subsubsection{Hodoscopes}

The measurement of particle trajectory in the spectrometer is primarily done by the hodoscope arrays. A single plane of hodoscopes is a set of long plastic scintillators, called fingers. stacked in a plane transverse to the particle trajectories. such that when a particle goes through that plane. one particular finger will produce a fast signal. The timing of this fast signal tells when the particle went through the struck finger.

An array of hodoscopes may have several planes aligned in different directions. so that by determining the overlap between the fingers struck at the same time. the particle location at the array may be determined. By placing a hodoscope array at the front and back of the spectrometer, two sets of position and time may be measured for each particle traveling through the spectrometer. By matching the events in the two hodoscope arrays, using knowledge of the particle's time of flight between the arrays, the particle's trajectory is determined. When the trajectory is combined with knowledge of the magnetic optics, the particle's track is reconstructed. The tracking information gives the particle momentum, scattering angle. and time of the scattering event with respect to the timing start signal.

The $2.75^{\circ}$ and $5.5^{\circ}$ hodoscope systems consisted of two hodoscope packages. one set located between the two Cherenkov tanks and one set located after the second 
Cherenkov tank. The first $2.75^{\circ}$ package held two $X$ planes, two $Y$ planes, a $U$ plane and a $V$ plane. The second $2.75^{\circ}$ package consisted of two $X$ and two $Y$ planes. The two $5.5^{\circ}$ packages each held one $X, Y, L$. and $V$ plane. The $X$ planes consist of fingers oriented vertically. thus determining horizontal position. The $Y$ planes determine vertical position using horizontal fingers. The $V^{*}$ and $V$ planes consist of fingers oriented at an angle with respect to the horizontal. The $U^{i}$ and $V$ planes in the front $2.75^{\circ}$ hodoscope package are respectively oriented at $+15^{\circ}$ and $-15^{\circ}$ to the horizontal. The $U$ and $V$ planes in both $5.5^{\circ}$ hodoscope packages were oriented at $+45^{\circ}$ and $-45^{\circ}$, respectively [23].

\subsubsection{Threshold Cherenkov Detectors}

The deep inelastically scattered electrons are not the only particles which enter the spectrometers. A reliable method of distinguishing electrons from the nonelectron background is required for the measurement of the electron DIS asymmetry. One way to do this is with a threshold Cherenkov detector.

A charged particle traveling through a medium with a velocity greater than the phase velocity of light in the medium $(c / n)$ will produce Cherenkov radiation. The minimum momentum at which a particle will exceed the phase velocity of light in the medium is given by:

$$
p_{\min } c=\frac{m c^{2}}{\sqrt{n^{2}-1}} .
$$

This minimum momentum. $p_{\min }$, depends upon the index of refraction of the material. $n$, and the mass of the particle, $m$. where $c$ is the speed of light in a vacuum. Particles with different masses will have different threshold momenta in a given medium.

The radiation is emitted at an angle with respect to the particle trajectory. $\theta_{\bar{C}}$. given by,

$$
\cos \theta_{C}=\frac{c}{v n}=\frac{c / n}{v}
$$


where $v$ is the particle velocity, $n$ is the index of refraction. and $c$ is the speed of light in a vacuum. One detail which has not been mentioned yet is that $n$ is frequency dependent. Since Cherenkov light is only emitted when $v \geq c / n(\omega)$. only regions of the frequency spectrum for which this is true will contribute to the Cherenkov light production. For different frequencies, the emission angle. $\theta_{\bar{C}}$, and threshold momentum. $p_{\min }$, will be different.

The number of photons of Cherenkov light produced over a distance $d x$ in a given frequency range is given by [81],

$$
\frac{d^{2} N}{d \omega d x}=\frac{z^{2} \alpha}{c} \frac{\left(v^{2}-\left(c / n\left(\omega^{\prime}\right)\right)^{2}\right)}{v^{2}}
$$

where $z$ is the particle charge in units of the electron charge. $\alpha$ is the fine structure constant. $v$ is the particle velocity, $\omega$ is the frequency, $n\left(\omega^{\prime}\right)$ is the index of refraction. and $c$ is the speed of light in a vacuum. Using the relativistic momentum. $p=$ $\frac{m v}{\sqrt{1-v^{2} / c^{2}}}$, and the threshold momentum as defined in Eq. 2.32. the distribution of photons may be rewritten as,

$$
\frac{d^{2} N}{d \omega d x}=\frac{z^{2} \alpha}{c}\left[1-\left(p / p_{\min }(\omega)\right)^{-2}\right]\left[1-(n(\omega))^{-2}\right]
$$

This equation is slightly misleading. because the $w$ dependence of the threshold momentum is due to its $n$ dependence. However, at a given value of $\omega$ and particle momentum, more massive particles (large $p_{\min }$ ) produce fewer photons than less massive particles (low $p_{\min }$ ). Fig. 2.14 shows that the Cherenkov light production reaches $90 \%$ of the maximum when the particle momentum is about three times the threshold momentum.

If, instead of the frequency dependence of the photon production. the dependence upon the wavelength in the medium is required, this may be derived using the relation, $\omega=c /(2 \pi n \lambda)$, which transforms Eq. 2.35 into,

$$
\frac{d^{2} N}{d \lambda d x}=\frac{z^{2} \alpha}{2 \pi n(\lambda) \lambda^{2}}\left[1-\left(p / p_{\min }(\lambda)\right)^{-2}\right]\left[1-(n(\lambda))^{-2}\right]
$$




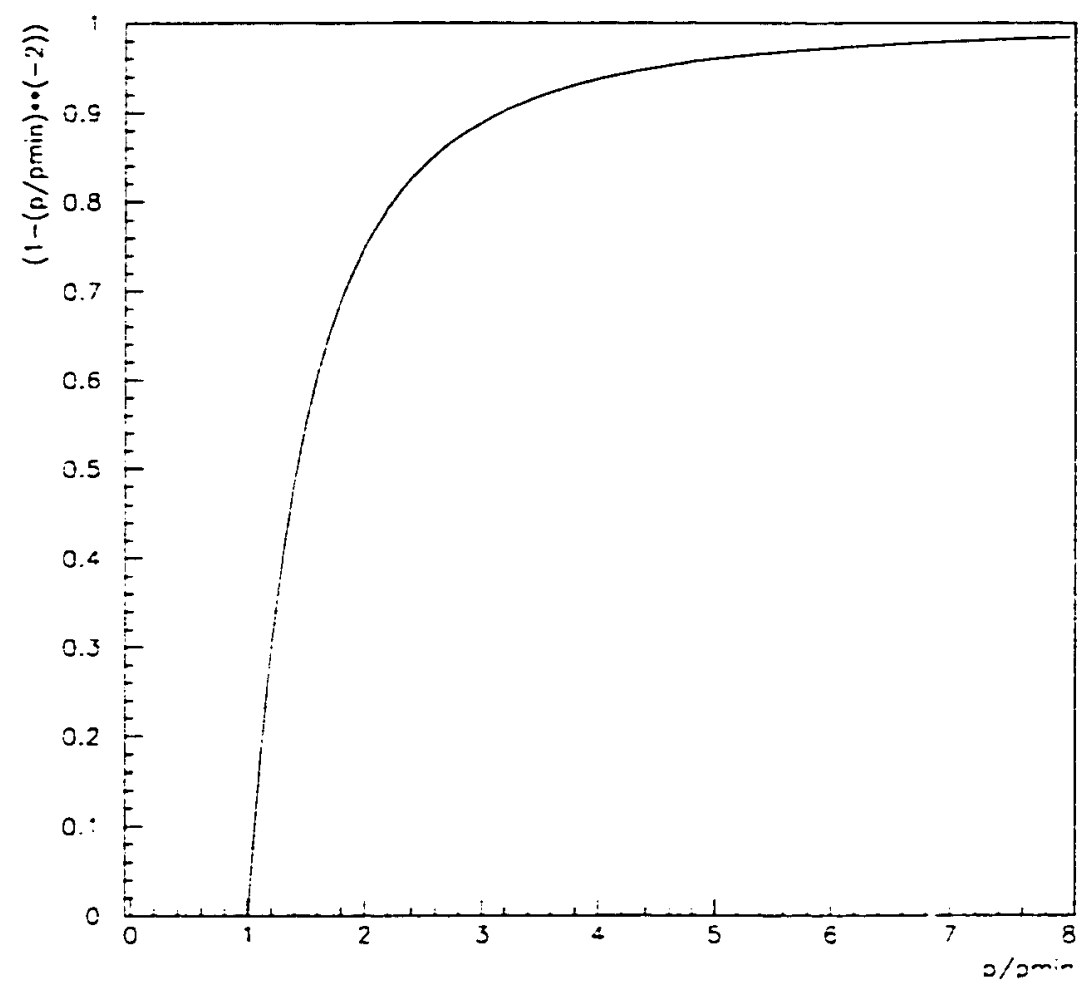

Figure 2.14: Plot of the momentum dependence of the Cherenkov light production (Eq. 2.35 ), with momentum in units of $p_{\min }$, at fixed values of $\omega$ and $n$.

where $\lambda$ is the wavelength of the light in the medium. and the index of refraction and threshold momentum have been reformed to depend upon $\lambda$. Since the production has a $\lambda^{-2}$ dependence and since $n(\lambda)$ goes to unity for short wavelengths, the Cherenkov light produced will be peaked in the ultraviolet.

In a threshold Cherenkov detector, the index of refraction is chosen such that the electron events produce a Cherenkov signal, but the non-electron particles either produce no Cherenkov signal, or a reduced signal. One way to choose an index of refraction is to choose the material. For gases, the index of refraction is related to the temperature and pressure of the gas. A gas-filled threshold Cherenkov detector can have the threshold adjusted by changing the pressure of the gas. For instance. nitrogen at a pressure of about $1 \mathrm{~atm}$ at $20^{\circ} \mathrm{C}$ has an index of refraction of about 1.0003 [82], which corresponds to a momentum threshold of about $20 \mathrm{MeV} / \mathrm{c}$ for electrons and about $6 \mathrm{GeV} / c$ for pions [83]. By reducing the pressure, the index of 
refraction decreases, and the threshold momentum increases.

The quantity of Cherenkov light produced is not the only important consideration when designing a Cherenkov detector. The light must be collected and converted into a electronic signal, and the Cherenkov signal produced should be stronger than other background signals, such as scintillation. For the Cherenkov light to be collected, the medium should not have a strong absorption in the ultraviolet, the light focusing system should transmit light into the ultraviolet, and the light detection device should be sensitive to as much of the ultraviolet as possible. Collimating baffles may be used to prevent non-Cherenkov light from entering the detector. The amount of scintillation light detected can be reduced by introducing a quenching gas into the medium.

Nitrogen gas is transparent to ultraviolet light of wavelength above about $140 \mathrm{~nm}$ [84. 82]. However oxygen and water vapor are strongly absorbing of light of wavelengths less than about $180 \mathrm{~nm}[8.5]$. Assuming the index of refraction is approximately constant over that range of wavelengths and integrating Eq. 2.36 to give light yield, the ratio in Cherenkov light transmission. for pure nitrogen and nitrogen with oxygen contamination, would be

$$
\frac{N_{N_{2}}}{N_{N_{2}, O_{2}}}=\frac{1 /(140 \mathrm{~nm})-1 / \lambda_{2}}{1 /(180 \mathrm{~nm})-1 / \lambda_{2}}
$$

where $\lambda_{2}$ is the upper edge of the wavelength sensitivity. The upper edge of the wavelength sensitivity for the E15.5 Cherenkov photomultiplier tubes was about 700 $\mathrm{nm}[8.5]$. Assuming the simple relation of Eq. 2.37 holds, the light collected with no $\mathrm{O}_{2}$ contamination would be 1.4 times the light collected with $\mathrm{O}_{2}$ contamination. In E154, a decrease in Cherenkov signals of 10 to $20 \%$ was noticed, due to $\mathrm{O}_{2}$ and $\mathrm{H}_{2} \mathrm{O}$ gradually leaking into the (sub-atmospheric pressure) tanks [8.5]. The Cherenkov tanks were refurbished before E155 to limit the leak rate, and thus the amount of oxygen contamination occurring over the duration of the experiment. The mirrors 
used in the E1.5.5 Cherenkov detectors had reflectivities of about $80-8.5 \%$ over the entire wavelength range [86]. The photomultiplier tubes used in the E1.5.5 Cherenkov detectors are not sensitive much below a wavelength of $300 \mathrm{~nm}$. To increase their sensitivity to the Cherenkov light, they were coated with p-terphenyl. which absorbs $\mathrm{CV}$ light and retransmits it in the range of $390 \mathrm{~nm}[8 \mathrm{i}]$. The spectral response of the coated photomultiplier tubes was not directly measured in E15.5, but in tests of similar tubes before E142. the coated photomultiplier tubes showed a gain increase of about $90 \%$ as compared to the uncoated tubes [88].

The $2.75^{\circ}$ and $5.5^{\circ}$ spectrometers each have a pair of gas threshold Cherenkov detectors, for redundancy in particle identification. The two Cherenkov detectors in the $2.75^{\circ}$ spectrometer were named " $2 \mathrm{C} 1 "$ and $" 2 \mathrm{C} 2 "$. and the $5.5^{\circ}$ detectors were named $" 5 \mathrm{Cl} "$ and $" 5 \mathrm{C} 2 "$. All four of the Cherenkov detectors are large aluminium vessels which were filled with a non-flammable mixture of $\mathrm{N}_{2}(90 \%)$ and $\mathrm{CH}_{4}(10 \%)$ gases. at an absolute pressure of about 0.1 atm. The primary Cherenkov medium is the $\mathrm{N}_{2}$ gas, while the admixture of $\mathrm{CH}_{4}$ gas serves to quench scintillation light produced in the nitrogen. At a pressure of about $0.1 \mathrm{~atm}$. the index of refraction of the $\mathrm{N}_{2} / \mathrm{CH}_{4}$ gas is about 1.00003 , yielding a pion threshold momentum of $18 \mathrm{GeV} / \mathrm{c}$. The Cherenkor light is collected by a set of concave mirrors at the downstream end of each tank, and is focused onto the face of a $5 "$ diameter photomultiplier tube. The Cherenkov anode signal is input to a flash ADC (FADC) which is used to extract the pulse height and timing information of the Cherenkov events.

The Cherenkov waveform was digitized by the FADC in approximately 1 ns time increments. For each time increment, the voltage of the Cherenkov signal is digitized into an 8-bit number. The FADC output is a listing of the voltage channel in each successive time increment. An average waveform corresponding to collection of Cherenkov light from an electron is shown in Fig. 2.15. The peak has a sharp rise and slower fall. The FADC analysis locates peaks with this structure within the 


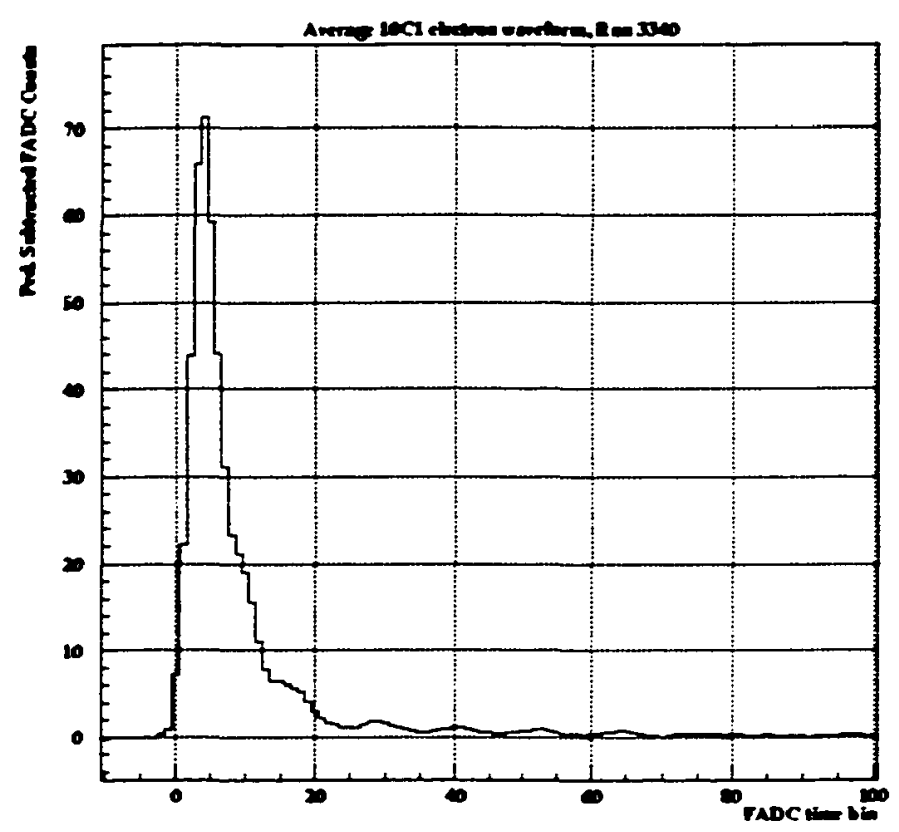

Figure 2.15: Average pedestal-subtracted Cherenkov waveform.

waveform: the timing is determined by finding the start time. and the peak height and integrated charge are also determined.

As a backup system, the last dynode signal from each tube is split into an $A D C$ and a set of four discriminators, from which signals are sent to TDC channels. The four discriminators have different thresholds, so by comparing which discriminators are fired for a given pulse, and the relative timing between discriminator signals. reconstruction of the pulse height into four bins is possible. This backup system was used to analyze part of the E155 production data, when the FADC instrumenting $5 \mathrm{C} 2$, the second Cherenkov detector in the $5.5^{\circ}$ spectrometer. temporarily failed.

\subsubsection{Electromagnetic Shower Calorimeter}

When a high energy electron encounters the electric field of a large nucleus it may undergo bremsstrahlung, where it radiates some of its energy in the form of a 
high energy photon. Similarly, when a high energy photon encounters the electric field of a large nucleus, it may produce an energetic electron-positron pair. The rate of both processes has a $\mathrm{Z}^{2}$ dependence, so a nucleus with a high atomic number interacts more effectively.

If a high energy electron encounters a material with a large density of high- $Z$ nuclei. these two processes result in an electromagnetic shower in the material. The initial electron undergoes bremsstrahlung. emitting a high energy photon. The photon pair-produces an electron and positron, both of which undergo bremsstrahlung. and the process continues. At each stage, the number of particles traversing the medium increases, and the average particle energy decreases. The average energy of an electron as it traverses the medium shows the exponential decay.

$$
E(x)=E_{0} \epsilon^{-x / L_{\text {rad }}}
$$

in which the radiation length. $L_{\text {rad }}$, is characteristic of the material and depends upon the atomic number and the density of atoms. The radiation length for a compound can be generated by finding the sum of the inverse radiation length of each element, weighted by the mass fractions: $\frac{1}{L_{\text {rad }}}=\frac{w_{1}}{L_{\text {rad.1 }}}+\frac{u_{1}}{L_{\text {rad.1 }}}+\ldots$ where the radiation length for each element is given by [81]

$$
\frac{1}{L_{\text {rad }}} \approx[4 Z(Z+1) N] r_{e}^{2} \alpha\left[\ln \left(183 Z^{-1 / 3}\right)-f(Z)\right]
$$

where $N=\frac{\rho N_{a}}{A}$ is the number density of atoms, $\rho$ is the density. $N_{a}=6.022 \times$ $10^{23} \mathrm{~mol}^{-1}$ is Avogadro's number, $A$ and $Z$ are the atomic weight and atomic number of the element, $f(Z)$ is a small Coulomb correction. $r_{e}=\epsilon^{2} /\left(m c^{2}\right)$ is the classical electron radius, and $\alpha$ is the fine structure constant. The shower process will continue until the electron energies, Eq. 2.38, decrease below the critical energy. $E_{c r i t}$. at which point the electron energy loss is dominated by ionization of the atoms in the material. A useful quantity to describe an electromagnetic shower in a material 
is the total shower track length. which is roughly the product of the total number of particles in the shower and the average distance traversed by a particle. If the full shower is contained within the material, then the total shower track length is proportional to the incident energy of the electron.

In a material which also has a high index of refraction, all of the electrons and positrons produced during the shower will be above the Cherenkov threshold. The amount of Cherenkov light produced is proportional to the total shower track length. so if the Cherenkov light can be collected and measured. the initial electron energy may be determined.

Another quantity which describes the electromagnetic shower is the shower maximum. The shower maximum. $t_{\max }$ is the depth of material at which the energy deposition in the material is at a maximum. and is given in units of radiation length by.

$$
t_{\text {max }}=\ln \frac{E_{0}}{E_{\text {crit }}}+C_{j} . \quad j=\epsilon . \uparrow .
$$

where $C_{e}^{\prime}=-0.5$ for electron induced showers. and $C_{\gamma}=0.5$ for photon induced showers [4].

Particles more massive than the electron are not as effective at producing an electromagnetic shower, because the bremsstrahlung rate depends upon $\mathrm{m}^{-4}$. so the pion and muon, having masses about 200 times the electron mass. would have bremsstrahlung production about $10^{-9}$ that of the electron. Additionally: the photon energy is substantially lower than the pion or muon energy. For the pion or muon, most particles will pass through the material, radiating only Cherenkov light proportional to their own path length through the material.

However, the pion may undergo hadronic showering or it may produce a knockon electron. In either case, the energy deposition will be greater than for a minimum ionizing particle, but may be distinguished from a scattered electron by comparing 
the deposited energy to the track momentum. An electron scattered into the spectrometer will have $E_{\text {deposited }} \approx P_{\text {track }}$, but a knock-on electron will be less energetic. and since the hadronic interaction length is longer than the electromagnetic radiation length, the hadronic showers will not be fully contained in the calorimeter. and will have a lower deposited energy. Neither pions nor muons will deposit all of their energy in the calorimeter.

The E1.5.5 electromagnetic shower calorimeters used glass with high lead content, which has a short radiation length (requiring less thickness of material to stop the electron showers) and a high index of refraction (producing strong Cherenkov light signals). The E1.5.5 calorimeters were segmented transversely into blocks. so that the position of the shower centroid could be determined.

The $2.75^{\circ}$ and $5.5^{\circ}$ spectrometer calorimeters were originally built for the $4.5^{\circ}$ and $7^{\circ}$ spectrometers of SLAC experiments E142/E143. Each is an array. 20 rows by 10 columns, of the $75 \mathrm{~cm} \times 6.2 \mathrm{~cm} \times 6.2 \mathrm{~cm}$ lead glass bars previously used in the $\mathrm{ASP}^{1}$ detector [89]. The bars are oriented with the $75 \mathrm{~cm}$ long axis parallel with the spectrometer axis, thus presenting a thickness of about 24 radiation lengths to the incident particles. The transverse segmentation of the calorimeters is such that electron showers typically are contained in a sub-array of nine blocks. Each of the blocks is instrumented with a $2^{*}$ photomultiplier tube affixed to the downstream end of the bar. The $5.5^{\circ}$ calorimeter PMT signals are split with one output being recorded by an $\mathrm{ADC}$ and the other sent to a discriminator. then input to a multihit TDC. Instead of one ADC and one TDC signal per block. the $2.755^{\circ}$ calorimeter PMT signals are split to provide one ADC and either two or three TDC signals. The 64 highest rate blocks in the $2.75^{\circ}$ calorimeter have three discriminator levels. and the remaining 136 blocks have two discriminator levels. Fig. 2.16 indicates which

\footnotetext{
${ }^{1}$ The ASP (Anomalous Single Photons) detector was designed to search for single photon events at the PEP efe- storage ring at SLAC.
} 


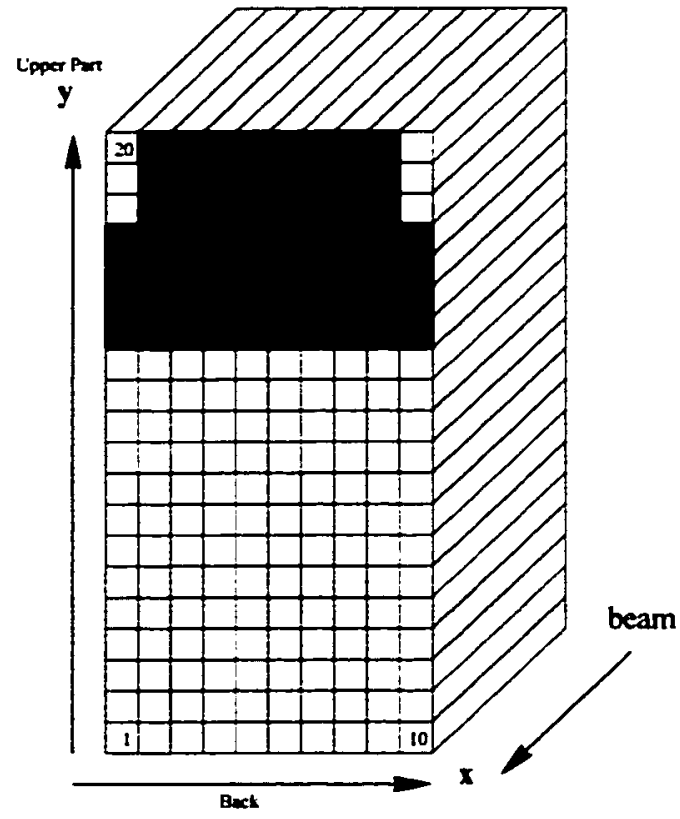

Figure 2.16: Diagram of the $2.75^{\circ}$ calorimeter blocks seen from the rear. The shaded blocks have three discriminator levels; the other blocks have two discriminator levels. Block numbering begins at the lower left corner, as seen from the rear of the calorimeter.

blocks have three levels of discrimination [90].

\subsection{Data Acquisition and Run Control}

E155 used a 'triggerless' data acquisition system. in which the electronics gate was generated by the accelerator synchronization signal, which is called the A2N signal. Since the accelerator repetition rate was at $120 \mathrm{~Hz}$ for most of E15.5. the data acquisition system needed to register the data from all of the electronics. often in excess of 6000 bytes of information per spill, at a repetition rate of $120 \mathrm{~Hz}$.

To accomplish this, E155 utilized a VME based data acquisition system [91]. Two CAMAC branches, containing the readout electronics for the three spectrometers, were connected to the Remote VME Real-Time System, which also contained the FADC modules for the five Cherenkov detectors. The Local VME Real-Time System read the CAMAC branch containing the beam instrumentation. Both the Local and Remote systems passed their data to the Local Data Server, which was 
a third VME crate containing UNIX processors. The Local Data Server sent the data to the Remote Data Server, which was responsible for logging the data to tape via the SLAC silo system. The Local Data Server also exported data via the SLAC Ethernet to workstations performing online analysis.

The data acquisition was controlled from DAQCtrl. The DAQCtrl system sent commands and received status messages from the Local and Remote Data Servers. displays experiment parameters obtained from ESA systems or MCC systems. and monitored warnings from the slow controls systems. Slow controls and monitoring was done on the ESA computer system with two batch processes, DAQJob and DAQMagb, which interacted with and were controlled by four interactive processes. DAQHV. DAQSclr. DAQLV, and DAQMagi. The four interactive processes were monitoring respectively the high voltage supplies, scalers. low voltage signals (such as CA.MAC and NIM crate power supplies), and the spectrometer magnets. The information from the slow controls systems were included in the data stream on a periodic basis.

\subsubsection{Data Stream - Event and Non-event Information}

There were two VME crates reading three C.AMAC branches into the event data stream. The remote VME crate, located in the $2.75^{\circ}$ hut. read the two CAMAC branches containing the $2.75^{\circ}$ and $5.5^{\circ} / 10.5^{\circ}$ modules. The local VME crate. located in CHA, read the CAMAC branch containing the beam electronics. During normal data collection, each of the modules in the two remote branches were read. and some of the beam modules were read. During Møller polarimeter data collection. additional beam modules were read, and the spectrometer modules were not.

Periodically during a run, a check-point occurred. During a checkpoint, the normal data acquisition stopped, and the data from the slow controls was inserted into the data stream. This included the ADC pedestals from the most recent pedestal 
run. magnet settings, and data from MCC pertaining to the beam. 


\section{CHAPTER 3}

\section{THE $10.5^{\circ}$ SPECTROMETER}

The $10.5^{\circ}$ spectrometer was constructed for E1.5.5 to increase the $Q^{2}$ range measured. This spectrometer can measure events with values $Q^{2}$ of up to eight times thai of the $2.75^{\circ}$ spectrometer. The design of the spectrometer had several constraints. Since the $2.75^{\circ}$ and $5.5^{\circ}$ spectrometers already existed on the experimental floor. the size and location of the new spectrometer was limited. resulting in a spectrometer significantly shorter in $Z$, the distance from the target along the spectrometer axis. Because the DIS differential cross-section drops precipitously with scattering angle. the $10.5^{\circ}$ spectrometer had to have the largest possible solid angular acceptance. By making the spectrometer shorter, a larger solid angle could be achieved by detectors of a given size. The $10.5^{\circ}$ spectrometer was not originally included in the E15.5 proposal. The addition of the large angle spectrometer was approved about six months before the experimental run. with only modest funding available. The spectrometer components were therefore constructed from existing detector systems or spare parts salvaged from the other two spectrometers.

During the E15.5 longitudinal running period. the particle rate in the $10.5^{\circ}$ spectrometer was about one pion per spill and one electron per 100 spills. Because the particle rate in the $10.5^{\circ}$ spectrometer is low, the detector packages can afford less redundancy than the other two spectrometers. which measure about 1 electron per spill in a background of multiple pions per spill. The small number of detector packages made this spectrometer much shorter than the other two. The $10.5^{\circ}$ spectrometer uses a single-bend magnet system, a single set of hodoscopes, one threshold 
Cherenkov detector, and a shower calorimeter consisting of a thin active preradiator and a total absorption calorimeter. The spectrometer acceptance is detector-limited. meaning that the magnetic optics will pass particles whose trajectories will not pass through all of the detectors.

\subsection{Spectrometer Coordinate System}

The spectrometer $Z$ axis is a line in the horizontal plane containing the beam line, which makes an angle of $-10.5^{\circ}$ with the beam line (on the right-ward side looking downstream). The spectrometer $Y$ axis is a vertical line, with positive $Y$ being up. and the $Y=0$ level being at the height of the beam line. The spectrometer $X$ axis is then a horizontal line, with positive $X$ being towards the beamline (in the $+\theta$ direction when the central axis is at $\left.\theta=-10.5^{\circ}\right)$. The relative scattering angles. $\Delta \theta$ and $\Delta \phi$, are defined with respect to the central trajectory of $\theta=-10.5^{\circ}$ and $0=0^{\circ}$. The $\Delta \theta$ axis is oriented with the positive $\Delta \theta$ direction in the same direction as the positive $\theta$ direction. so a positive $\Delta \theta$ is a smaller scattering angle. The $\Delta_{0}$ axis is oriented in the same direction as the $\phi$ axis. A positive $>0$ scattering angle is a trajectory scattered upward. By common usage. the "high $\theta$ side of the $10.5^{\circ}$ spectrometer has the larger scattering angle ( $\Delta \theta$ is negative). and the "low $\theta$ side has the smaller scattering angle ( $\Delta \theta$ is positive).

Both before and after the experimental run, the spectrometer hut was surveyed to determine the location of all the detector elements in the spectrometer coordinate system. The $10.5^{\circ}$ shower calorimeter survey data from 9 May 1997 is listed in Table 3.1 .

\subsection{Magnetic Optics and Collimation}

The $10.5^{\circ}$ spectrometer uses a quadrupole-dipole-quadrupole system of magnets. This configuration allowed the spectrometer to have a large acceptance. by 


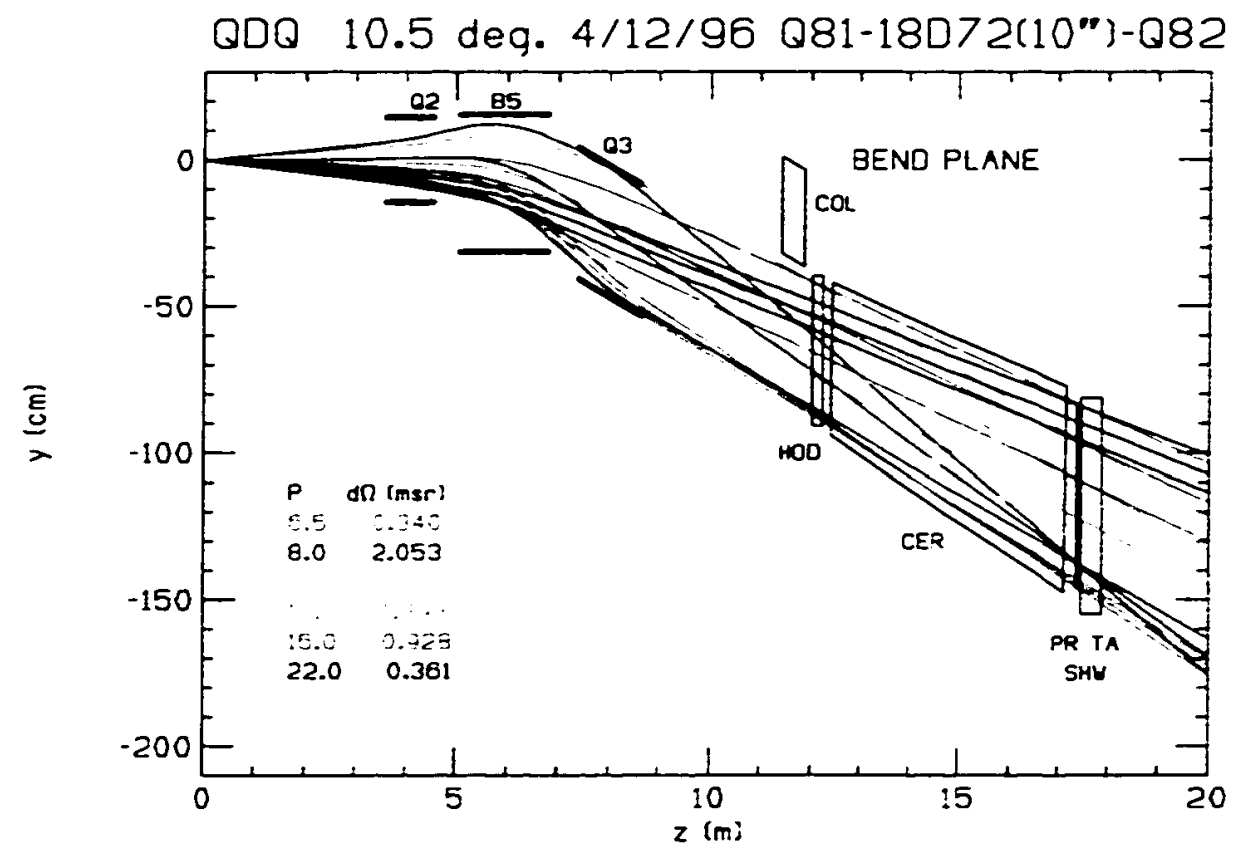

Figure 3.1: View of the bend plane of the $10.5^{\circ}$ spectrometer optics model, showing various trajectories through the spectrometer components. The trajectories are the high $\phi$, low $\phi$. and central $\phi$ trajectories for six different momenta. The solid angles accepted for each momentum are: $d \Omega(6.5 \mathrm{Gev})=0.340 \mathrm{msr} ; d \Omega(8.0 \mathrm{Gev})=2.053 \mathrm{msr} ; d \Omega(10.0 \mathrm{Gev})=$ $1.637 \mathrm{msr} ; d \Omega(12.0 \mathrm{Gev})=1.393 \mathrm{msr} ; d \Omega(16.0 \mathrm{Gev})=0.928 \mathrm{msr} ; d \Omega(22.0 \mathrm{Gev})=0.361$ msr.

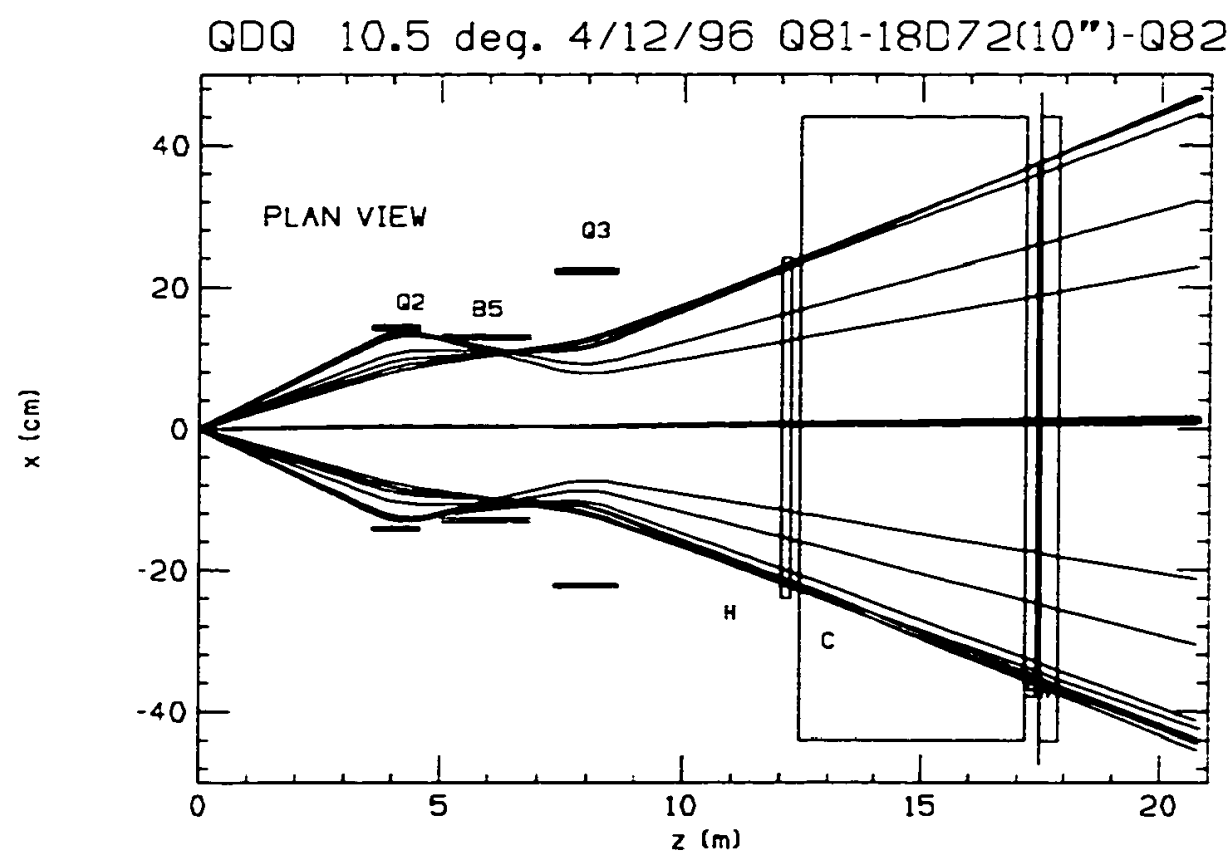

Figure 3.2: View of the non-bend plane of the $10.5^{\circ}$ spectrometer optics model, showing assorted trajectories. The trajectories are the high $\theta$, low $\theta$, and central $\theta$ trajectories for the six momenta shown in Figure 3.1. 


\begin{tabular}{|lcc|}
\hline & $X$ Position (in.) & $X$ position $(\mathrm{cm})$ \\
\hline Baseplate. US, $-X$ & -24.200 inch & $-61.468 \mathrm{~cm}$ \\
Baseplate, DS, $-X$ & -23.985 inch & $-60.922 \mathrm{~cm}$ \\
TA Stack Seam 4/5 & -17.370 inch & $-44.120 \mathrm{~cm}$ \\
TA Stack Seam 3/4 & -17.433 inch & $-44.280 \mathrm{~cm}$ \\
TA Stack Seam 2/3 & -17.463 inch & $-44.356 \mathrm{~cm}$ \\
TA Stack Seam 1/2 & -17.562 inch & $-44.607 \mathrm{~cm}$ \\
\hline \hline & $Y$ Position (in.) & $Y$ position $(\mathrm{cm})$ \\
\hline Baseplate. US. $-X$ & -60.793 inch & $-154.41 \mathrm{~cm}$ \\
Baseplate, DS, $-X$ & -60.830 inch & $-1.54 .51 \mathrm{~cm}$ \\
Baseplate. DS, $+X$ & -61.008 inch & $-1.54 .96 \mathrm{~cm}$ \\
PR Stack Top, $-X$ & -32.910 inch & $-83.591 \mathrm{~cm}$ \\
PR Stack Top, $+X$ & -32.970 inch & $-83.744 \mathrm{~cm}$ \\
TA Stack Seam 4/5 & -37.570 inch & $-95.428 \mathrm{~cm}$ \\
TA Stack Seam 3/4 & -43.430 inch & $-110.31 \mathrm{~cm}$ \\
TA Stack Seam 2/3 & -49.230 inch & $-125.04 \mathrm{~cm}$ \\
TA Stack Seam 1/2 & -55.066 inch & $-139.87 \mathrm{~cm}$ \\
\hline
\end{tabular}

Table 3.1: $X$ and $Y$ survey data for the 9 May 1997 survey of the shower calorimeter.

permitting the spectrometer to be very close to the target. and focusing the trajectories horizontally. The maximum horizontal acceptance was \pm 34 milliradians. and the maximum vertical acceptance was \pm 18 milliradians. The first quadrupole magnet. Q2, which was Q81 in the SLAC $8 \mathrm{GeV}$ spectrometer [92]. focuses the particle trajectories horizontally, increasing the range in horizontal scattering angle accepted by the spectrometer. The dipole is a standard copper coil 18D 72 magnet $^{1}$ with the gap enlarged from 6 inches to 10 inches. The gap is shimmed at the top with wood, and at the bottom with stainless steel [79]. The dipole magnet bends the particle trajectories downward, deflecting the central trajectory by 100 mrad. The final quadrupole, Q3, which was $\mathrm{Q} 82$ in the $8 \mathrm{GeV}$ spectrometer. focuses vertically, and defocuses horizontally. Together, the magnetic optics system is strongly focusing for particles of momentum less than $10 \mathrm{GeV} / c$. At $6 \mathrm{GeV} / c$. the focal point is inside the Cherenkov tank, while $8 \mathrm{GeV} / c$ particles have a focus at the

\footnotetext{
${ }^{1}$ An $18 D 72$ magnet is a dipole magnet 72 inches long, having a pole face 18 inches wide, and a gap between pole faces of 6 inches.
} 
shower calorimeter. The system is nonconvergent for particles more energetic than $10 \mathrm{GeV} / \mathrm{c}[\overline{79}$ ]. Figures 3.1 and 3.2 show particle trajectories calculated using the optics model, for the bend plane and non-bend plane of the spectrometer. The magnets were initially operated with a central momentum of $12 \mathrm{GeV} / c$, but the central momentum was reset to $11.7 \mathrm{GeV} / c$ very early in the experiment, because the magnet power supplies could not remain steady at the higher current setting.

A lower lip collimator is situated before Q2. at a $Z$ position of about $3.55 \mathrm{~m}$ and the top is at a $Y$ position of about $-6.4 \mathrm{~cm}$. This lead and tungsten collimator blocked particles with a downward $\phi$ deflection of greater than $18 \mathrm{mrad}[79]$. This was a physical acceptance limit. rather than a limit on the vertical scattering angle of the electrons. due to the effects of the target magnetic field. The tracking matrix needed to include the target magnetic field in addition to the spectrometer magnets.

An "eyebrow" collimator. designed to block neutral particles. is situated after Q3. just inside the detector hut and positioned above the spectrometer acceptance. Because the $10.5^{\circ}$ spectrometer had only a single shallow bend. a line-of-sight path existed from the target into the spectrometer hut. The eyebrow collimator served to block this direct path for neutral particles produced at the target. The collimator is otherwise out of the spectrometer acceptance. It is a large block of lead. $48 \mathrm{~cm}$ wide. $33 \mathrm{~cm}$ tall, and $46 \mathrm{~cm}$ thick. It is tilted from the horizontal by $-100 \mathrm{mrad}$ to be parallel to the central ray in the spectrometer. The forward edge is at a spectrometer coordinate of $Z=1140 \mathrm{~cm}[93]$.

During most of E15.5, a "low theta scraper" (LTS) was employed to reduce the background rate, by blocking the lowest $19 \mathrm{mrad}$ of the $\theta$ acceptance (highest 19 mrad in $\Delta \theta$ ). The LTS was installed on top of the lower lip collimator. in front of Q2. The LTS was first implemented for Run 570. and was used throughout the data collection for the longitudinal asymmetry and some of the transverse asymmetry measurement. It was removed for Run 3790, and the final transverse asymmetry 


\begin{tabular}{|clcccc|}
\hline Run Period & Designation & $+\Delta \theta$ & $-\Delta \theta$ & $+\Delta o$ & $-\Delta o$ \\
\hline $478-569$ & Letterbox & $34 \mathrm{mrad}$ & $-34 \mathrm{mrad}$ & $1.0 \mathrm{mrad}$ & $-2.8 \mathrm{mrad}$ \\
$570-6.57$ & Low Theta Scraper & $15 \mathrm{mrad}$ & $-34 \mathrm{mrad}$ & $18 \mathrm{mrad}$ & $-18 \mathrm{mrad}$ \\
$6.58-746$ & Jailbars: Gap 1 & $15 \mathrm{mrad}$ & $11 \mathrm{mrad}$ & $18 \mathrm{mrad}$ & $-18 \mathrm{mrad}$ \\
& Gap 2 & $-3.8 \mathrm{mrad}$ & $-7.6 \mathrm{mrad}$ & $18 \mathrm{mrad}$ & $-18 \mathrm{mrad}$ \\
& Gap 3 & $-23 \mathrm{mrad}$ & $-34 \mathrm{mrad}$ & $18 \mathrm{mrad}$ & $-18 \mathrm{mrad}$ \\
$748-1021$ & Letterbox 2 & $11 \mathrm{mrad}$ & $-34 \mathrm{mrad}$ & $0.5 \mathrm{mrad}$ & $-2.8 \mathrm{mrad}$ \\
$1022-2818$ & Low Theta Scraper & $15 \mathrm{mrad}$ & $-34 \mathrm{mrad}$ & $18 \mathrm{mrad}$ & $-18 \mathrm{mrad}$ \\
$2819-2853$ & Jailbars: Gap 1 & $15 \mathrm{mrad}$ & $3.8 \mathrm{mrad}$ & $11 \mathrm{mrad}$ & $-3.7 \mathrm{mrad}$ \\
& Gap 2 & $-3.8 \mathrm{mrad}$ & $-1.5 \mathrm{mrad}$ & $11 \mathrm{mrad}$ & $-3.7 \mathrm{mrad}$ \\
& Gap 3 & $-23 \mathrm{mrad}$ & $-34 \mathrm{mrad}$ & $11 \mathrm{mrad}$ & $-3.7 \mathrm{mrad}$ \\
$2854-32.7$ & Low Theta Scraper & $1.5 \mathrm{mrad}$ & $-34 \mathrm{mrad}$ & $18 \mathrm{mrad}$ & $-18 \mathrm{mrad}$ \\
$3234-3252$ & Sieve Slits & Restricted to four holes. & \\
$3253-3.357$ & Low Theta Scraper & $15 \mathrm{mrad}$ & $-34 \mathrm{mrad}$ & $18 \mathrm{mrad}$ & $-18 \mathrm{mrad}$ \\
$3358-3404$ & Sieve Slits & Sieve with LTS & & \\
$3405-3781$ & Low Theta Scraper & $15 \mathrm{mrad}$ & $-34 \mathrm{mrad}$ & $18 \mathrm{mrad}$ & $-18 \mathrm{mrad}$ \\
$3782-3786$ & Sieve Slits & Sieve with LTS & & \\
$3790-4384$ & & $34 \mathrm{mrad}$ & $-34 \mathrm{mrad}$ & $18 \mathrm{mrad}$ & $-18 \mathrm{mrad}$ \\
\hline
\end{tabular}

Table 3.2: Collimation periods for the $10.5^{\circ}$ spectrometer. The limits shown are the maximum range for all particles. The acceptance deviated from these values depending upon particle momentum.

runs were taken with the full $\theta$ acceptance.

Several times during the experiment. additional collimation was employed to restrict the horizontal or vertical acceptance for calibration purposes. Table 3.2 shows the maximum angular acceptances for the spectrometer throughout E1.5.5. There were three types of collimation modes: "letterbox". -jailbar". or "sieve slit". All three types were installed on the lower lip collimator in front of Q2. The letterbox and jailbar modes were built from lead bricks stacked to either restrict the 0 acceptance in the letterbox mode, or restrict the $\theta$ acceptance in jailbar mode. The sieve slit is a 1 -inch thick tungsten plate with five rows of seven holes spaced evenly in the vertical and horizontal directions. The holes are about $7 \mathrm{~mm}$ in diameter. and have a horizontal spacing of about $3.5 \mathrm{~cm}$ and a vertical spacing of about $2.8 \mathrm{~cm}$. The purpose of the sieve slit was to provide very narrow definitions of the electron trajectories for evaluation of the optics model used by the tracking reconstruction. 


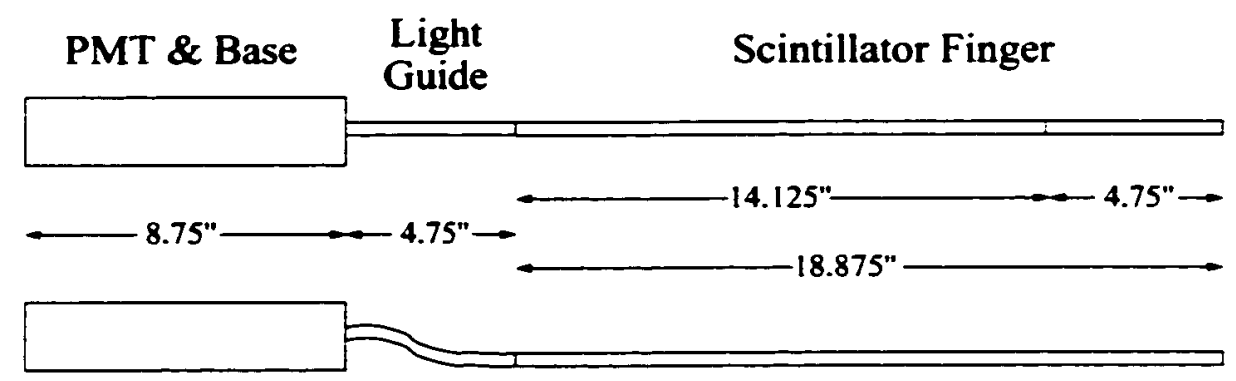

Figure 3.3: Side view diagram of the $10.5^{\circ}$ hodoscope straight and bent fingers.

\section{$3.3 \quad$ Hodoscopes}

During E15.5, the $10.5^{\circ}$ spectrometer had a single set of hodoscopes located at the front of the spectrometer hut. All four planes were oriented horizontally. to provide a $Y$ coordinate measurement at the front of the spectrometer. The hodoscope consisted of four planes each containing eight 2-inch fingers. with a gap of 0.5 inches between adjacent fingers. The planes are separated by 2.875 inches in $Z$. The downstream planes are offset downward by 0.62 .5 inches compared to the preceding plane [94]. Table 3.3 shows the locations of each hodoscope finger in the spectrometer coordinate system. Each scintillator was coupled via a light guide to a 2-inch Hamamatsu R329-02 photomultiplier tube. Two types of fingers were used. fingers with straight light guides and fingers with bent light guides. Figure 3.3 shows the side profile of both types of fingers. Eighteen of the fingers have an S-curve light guide: the other fourteen fingers have straight light guides. Both types of light guide extend 4.75 inches from the face of the photomultiplier tube. Both types of fingers have a scintillator 18.875 inches long, but the straight fingers are built from an original scintillator length of 14.125 inches, with a 4.75 inches extension [94]. The photomultiplier tubes are protected from stray magnetic fields by a $\mu$-metal shield. In the original installation, the $\mu$-metal shields did not extend beyond the PMT face, resulting in PMT gain shifts as discussed in Section 5.3.1. Therefore. the $\mu$-metal shields were modified during the E15.5 experiment to better protect the 


\begin{tabular}{|c|c|c|c|c|}
\hline Plane & Finger & $Y_{\text {center }}(\mathrm{mm})$ & $Z(\mathrm{~mm})$ & HV Setting (V) \\
\hline 1 & 1 & -406.4 & 12030.64 & 1375 \\
\hline 1 & 2 & -469.9 & 12030.64 & 1425 \\
\hline 1 & 3 & -.533 .4 & 12030.64 & $1: 300$ \\
\hline 1 & 4 & -.596 .9 & 12030.64 & 1250 \\
\hline 1 & 5 & -660.4 & 12030.64 & $1: 3.50$ \\
\hline 1 & 6 & -723.9 & 12010.00 & 1300 \\
\hline 1 & $\tau$ & -787.4 & 12010.00 & $1: 300$ \\
\hline 1 & 8 & -8.50 .9 & 12010.00 & 1400 \\
\hline 2 & 1 & -422.4 & 12100.64 & 13.55 \\
\hline 2 & 2 & -485.9 & 12100.64 & $1: 375$ \\
\hline 2 & 3 & -.549 .4 & 12100.64 & $1: 350$ \\
\hline 2 & 4 & -612.9 & 12100.64 & 1500 \\
\hline 2 & 5 & -676.4 & 12100.64 & 1400 \\
\hline 2 & 6 & -739.9 & 12080.00 & 1375 \\
\hline 2 & $\tau$ & -803.4 & 12080.00 & 1350 \\
\hline 2 & 8 & -866.9 & 12080.00 & 1305 \\
\hline 3 & 1 & -438.4 & 12180.64 & $1: 300$ \\
\hline 3 & 2 & -.501 .9 & 12180.64 & 1375 \\
\hline 3 & 3 & -.56 .5 .4 & 12180.64 & 1400 \\
\hline 3 & 4 & -628.9 & 12180.64 & 1425 \\
\hline 3 & 5 & -692.4 & 12160.00 & $1: 350$ \\
\hline 3 & 6 & -75.5 .9 & 12160.00 & 1400 \\
\hline 3 & $i$ & -819.4 & 12160.00 & 1325 \\
\hline 3 & 8 & -882.9 & 12160.00 & 1350 \\
\hline 4 & 1 & -4.53 .4 & 12250.64 & 1325 \\
\hline 4 & 2 & -.516 .9 & 12250.64 & 1350 \\
\hline 4 & 3 & -580.4 & 12250.64 & $1: 300$ \\
\hline 4 & 4 & -643.9 & 12250.64 & 1375 \\
\hline 4 & 5 & -707.4 & 12230.00 & 1475 \\
\hline 4 & 6 & -770.9 & 12230.00 & 142.5 \\
\hline 4 & 7 & -834.4 & $122: 30.00$ & 1500 \\
\hline 4 & 8 & -897.9 & 12230.00 & 1450 \\
\hline
\end{tabular}

Table 3.3: Locations of the $10.5^{\circ}$ hodoscope fingers within the spectrometer coordinate system. All fingers are centered horizontally at $X=0.0 \mathrm{~mm}$ with a width of $\Delta X=$ $240 \mathrm{~mm}$. All fingers have a vertical width $\Delta Y=25.4 \mathrm{~mm}$. The high voltage setting. as of Run 4260 , for each finger is also shown. 
PMTs.

Each of the hodoscope PMTs had high voltage supplied to it from an individual channel of a LeCroy HV4032A high voltage chassis. The signal from each PMT was input to a channel of a LeCroy 3412 discriminator with an output width of $6 \mathrm{~ns}$. then sent into a LeCroy $337 \mathrm{i}$ multihit TDC operating in leading-edge only mode.

\subsection{Threshold Cherenkov Detector}

The threshold Cherenkov detector in the $10.5^{\circ}$ spectrometer is a tank almost $5 \mathrm{~m}$ long, with an inner diameter of 36 inches upstream, and an inner diameter of 50 inches downstream. The forward and rear windows are hydro-formed aluminum which is 63 mils thick [9.5]. The active length was about $4.7 \mathrm{~m}$ [60]. Located just forward of the rear window, a set of two curved mirrors focuses the Cherenkov light down onto a 5-inches photomultiplier tube. The PMT is sheltered from stray particles within a hut of lead bricks, which provided 8 inches shielding upstream. 4 inches on the sides, and 2 inches on the top.

The $10.5^{\circ}$ Cherenkov tank used two $44 \mathrm{~cm}$ by $70 \mathrm{~cm}$ mirrors placed side by side. creating a light collection area approximately $88 \mathrm{~cm}$ wide by $70 \mathrm{~cm}$ tall [79]. The center of the mirror system is at $X=0.0 \mathrm{~mm} . Y=-1125.0 \mathrm{~mm}$. and $Z=16850.0 \mathrm{~mm}$ in the spectrometer coordinates. The center of the PMT is at approximately $X=0.0 \mathrm{~mm}, Y=-160.5 .0 \mathrm{~mm}$, and $Z=16.350 .0 \mathrm{~mm}$ in the spectrometer coordinates [96], giving a center to center separation between the mirrors and PMT of approximately $693 \mathrm{~mm}$.

The Cherenkov tank was filled with a mixture of $\mathrm{N}_{2}$ and $\mathrm{CH}_{4}$ gas at a pressure of 1.91 psia for Run 600 through Run 2102, and 2.87 psia after Run 2111 [97]. The nitrogen was mixed with methane $\left(90 \% \mathrm{~N}_{2} / 10 \% \mathrm{CH}_{4}\right)$ to quench the scintilla-

tion light, as discussed in Section 2.4.3. The pressure was increased to provide a greater separation between the electron peak and the single-photoelectron peak in 
the Cherenkov spectrum.

The Cherenkov PMT high voltage was set to $+2210 \vee$ throughout E15.5 [97]. The PMT was a Hamamatsu R1584 tube. the face of which was coated with wavelength shifting material, as discussed in Section 2.4.3.

The upper plot in Figure 3.4 shows an average of the detected peaks in the $10.5^{\circ}$ Cherenkov detector for a typical run. The Cherenkov peaks are fairly fast. with the bulk of the peak contained within $10 \mathrm{~ns}$. The analysis routine locates peaks of this form in the raw FADC spectrum, and determines the peak time as the beginning of the peak. The lower plot, also from Run 3340, in Figure 3.4 shows the single photoelectron peak, and the broad distribution resulting from the Cherenkov radiation of an electron in the detector; the peak corresponds to about $i$ photo-electrons collected by the PMT [98].

The Cherenkov detector was instrumented as in the other spectrometers. with the anode signal input to the FADC and the dynode signal used for the backup system. The dynode signal was fanned out, using a LeCroy $428 F$ FIFO, to an ADC channel and two discriminator channels in a Lecroy 3412 CAMAC discriminator. One channel used the signal direct from the fan-out, and the other channel used the signal after an attenuator. The signals from the discriminator channels were input to twelve channels of a LeCroy 3377 TDC via a LeCroy 227\%/EXP Hit Expander. The low threshold (non-attenuated) discriminator channel only provided leading-edge timing ( 4 TDC channels). The high threshold (attenuated) discriminator channel provided both leading-edge ( 4 TDC channels) and trailing-edge timing (4 TDC channels) [99].

\subsection{Lead Glass Electromagnetic Shower Calorimeter}

The $10.5^{\circ}$ shower counter consisted of a 2 radiation length preradiator and a 1.5-16 radiation length total absorber. The preradiator was segmented vertically into 

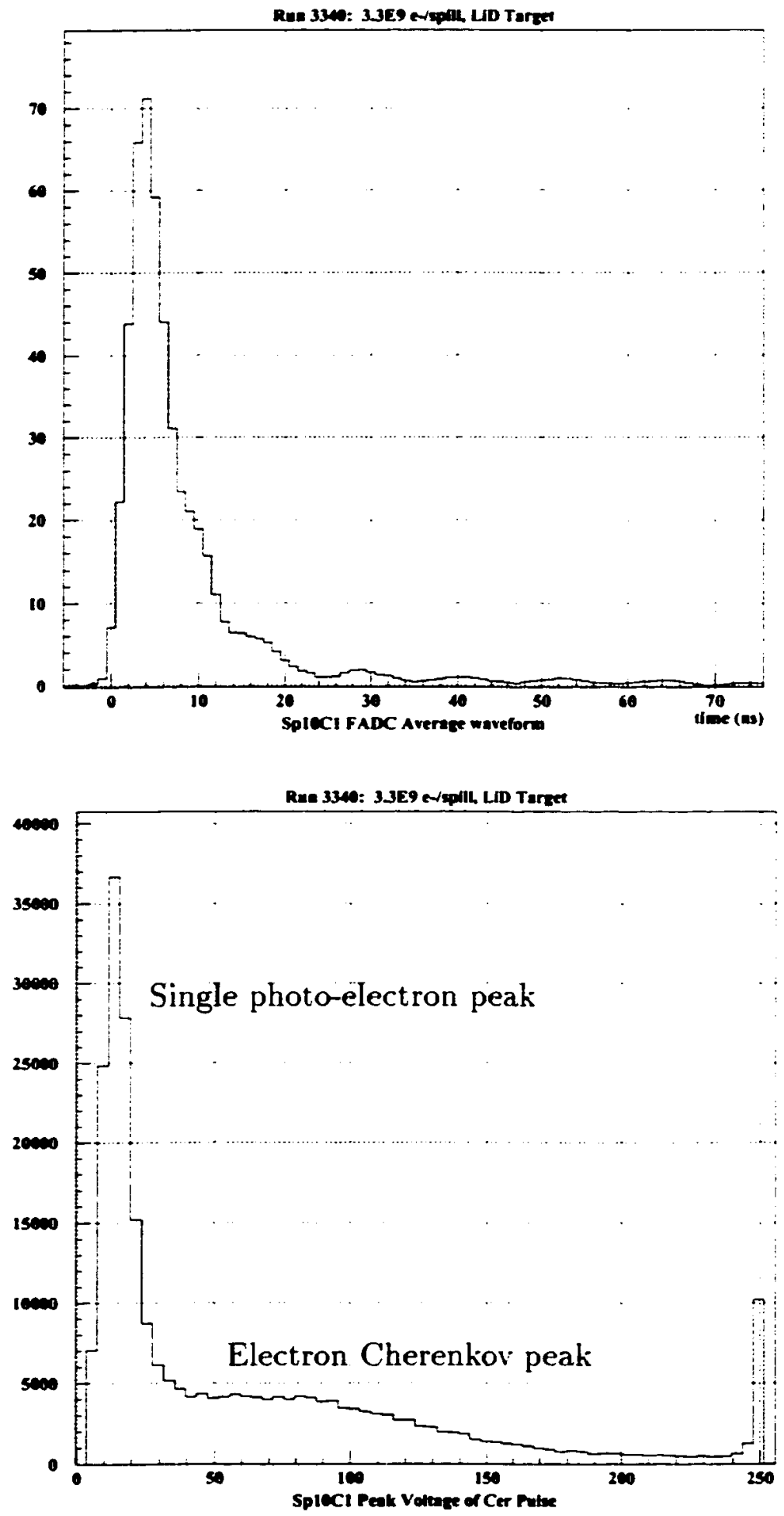

Figure 3.4: Cherenkov average waveform and spectrum of $V_{\text {peak }}$ for Run 3340. The Cherenkov detector pressure is $2.8 \mathrm{i}$ psia; so the electron Cherenkov peak corresponds to about 7 photo-electrons [98]. 


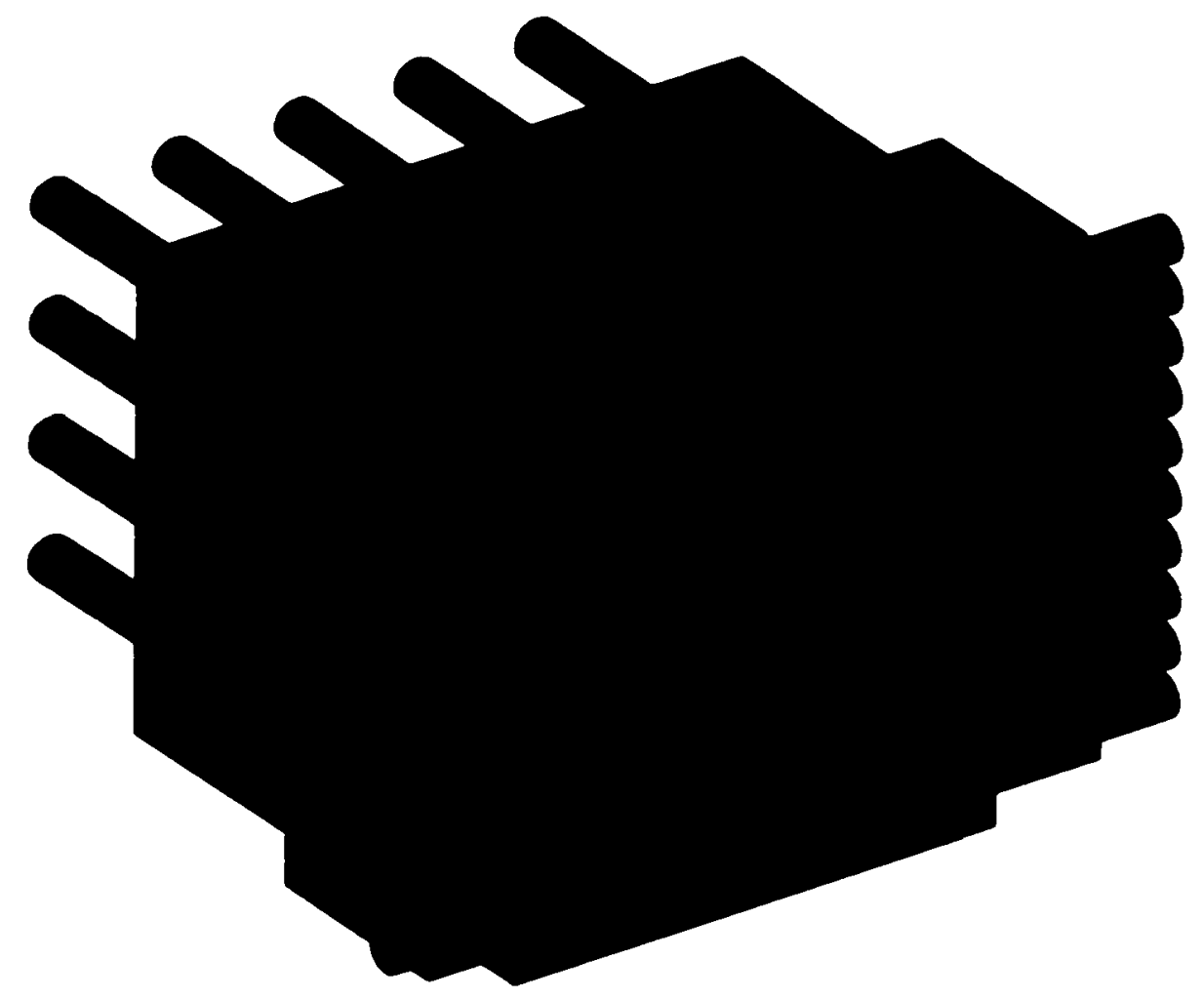

Figure 3.5̃: Drawing of the $10.5^{\circ}$ shower calorimeter, showing the TA blocks and PR bars.

ten horizontal bars, each bar having a photomultiplier tube at either end. The total absorber consisted of 30 blocks, arranged in a stack six blocks wide by five blocks tall. Figure 3.5 is a diagram of the calorimeter used for a GEA.NT simulation, showing the relative positions of the total absorber blocks and the preradiator bars.

The preradiator and total absorber stacks each had an independent test and calibration signal system. For both stacks, the system was an LED inserted into the common end of a yoke of forty-two bundles of optical microfibers. The microfibers contained in a particular bundle are not grouped together at the yoke. The individual bundles made contact with the lead glass of a block through a bracket, or "gizmo". The gizmo served as an anchor point for the fiber so that it will maintain contact with the lead glass. The unused bundles of each yoke were taped off. 


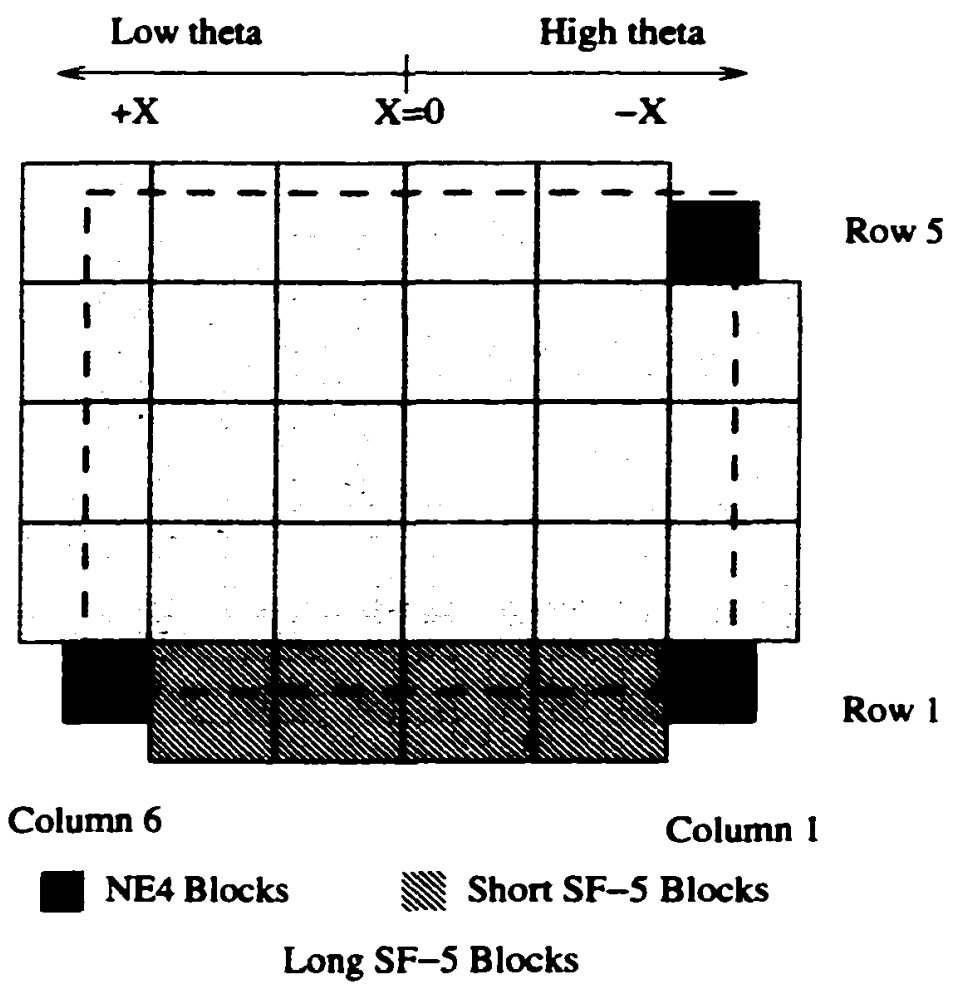

Figure 3.6: Diagram of the TA block faces, looking downstream. The three types of blocks used for the total absorber are indicated. The heavy dashed line indicates the outer edge of the preradiator layer. All scattered electrons passing through the preradiator layer will be contained in the total absorber layer.

\subsubsection{Total Absorber}

There were three types of total absorber (TA) blocks used in the $10.5^{\circ}$ shower counter. Of the thirty blocks in the TA, twenty-seven were used in the $8 \mathrm{GeV}$ spectrometer calorimeter and three were used in the NE4 calorimeter [100]. Four of the blocks from the $8 \mathrm{GeV}$ spectrometer calorimeter were shorter than the remaining twenty-three. The three .NE4 blocks were shorter and had a smaller transverse area than both sizes of the $8 \mathrm{GeV}$ spectrometer blocks. The relative positions of the three types of blocks are shown in Fig. 3.6.

The 27 large blocks all had a height and width of $14.6 \mathrm{~cm}$. They are SF-5 [101] type lead glass with an index of refraction of 1.67 , a critical energy of about $16 \mathrm{MeV}$, and an estimated radiation length ${ }^{2}$ of $2.47 \mathrm{~cm}$. Each of these blocks was

\footnotetext{
${ }^{2}$ The radiation length of this glass was not clearly documented. Several references $[102,103$,
} 
instrumented with a XP2041/00 type 5-inch diameter photomultiplier tube. Four of the blocks had a depth of $35 \mathrm{~cm}$, while the other 23 had a depth of $40 \mathrm{~cm}$. corresponding to thicknesses of 14.2 and 16.2 radiation lengths, respectively. The four short blocks were the center four blocks in the bottom row. The bottom row of the total absorber accepted particles with momentum less than about $8 \mathrm{GeV} / \mathrm{c}$. so the shorter blocks provided sufficient material to contain these electron showers.

Three of the corners of the total absorber were the NE4 blocks. the two bottom corners and the upper corner on the high scattering angle edge. as shown in Fig. 3.6. These blocks had a height and width of $10.5 \mathrm{~cm}$. and a depth of $25 \mathrm{~cm}$. These blocks were SF-6 type lead glass, with an index of refraction of 1.81. a radiation length of $1.69 \mathrm{~cm}[104,101]$, and a thickness of 14.8 radiation lengths. These blocks were instrumented with Hamamatsu 1911-05 hybrid assembly photomultipliers. affixed to the rear of the block.

The signals from each TA photomultiplier tube were split in two. using passive splitters. One signal was passed to a LeCroy $2249 \mathrm{~W}$ ADC. and the other was passed to a LeCroy 623B discriminator and then to a LeCroy 2277 TDC operated in leadingedge/trailing-edge mode. However, the $623 \mathrm{~B}$ discriminator could not operate in a time-over-threshold mode, so the trailing-edge timing information was not useful for the analysis (see Section 5.3.2).

\subsubsection{Preradiator}

The $10.5^{\circ}$ preradiator consisted of 10 bars of F-2 type lead glass doped with cerium. They were previously used in the ASP detector [89], and other bars of this type were used in the $2.75^{\circ}$ and $5.5^{\circ}$ spectrometer shower counters [109]. Each bar was $7.5 \mathrm{~cm}$ long, with a height and thickness of about $6.2 \mathrm{~cm}$. They were stacked 104. 101, 105, 106, 107. 108] were consuited, some of which disagreed as to what type of glass the blocks were, or on the composition of the various types of glass. Eventually the value of $2.47 \mathrm{~cm}$ was decided upon as a central value, for the purposes of simulation. 
vertically with their long axis oriented horizontally, transverse to the spectrometer axis. These bars have a radiation length of $3.17 \mathrm{~cm}$ [89]. so the preradiator is 1.96 radiation lengths thick. The index of refraction is 1.58 . and the density is $3.61 \mathrm{~g} / \mathrm{cm}^{3}$.

Each bar had an RCA 8.575 photomultiplier tube affixed to each end. Since these $\mathrm{PR}$ bars are known to have a significant attenuation of light along their length, it was planned to use the ADC signals from each end to estimate the location of the hit. By comparing the ADC signal strengths and the timing differences between the ends of each bar two possible determinations of the strike location may be made for each PR event. The best estimate for the attenuation length in the preradiator bars was about 1 meter ${ }^{3}$.

The photomultiplier signals from the PR bars were fanned-out using LeCroy 428 F FIFO. with two output channels going to a LeCroy 2249W ADC and a LeCroy 623B discriminator. The discriminator signal was input to a LeCroy 337 TDC operated in leading-edge mode.

After the total absorber blocks had been stacked and secured, the preradiator blocks were stacked directly in front of the TA. The PR stack was centered vertically about $2 \mathrm{~cm}$ above the center of the TA stack. This was accomplished by using a large aluminum bar, 3.4-inch $\mathrm{H} \times 2.4$-inch $\mathrm{D} \times 3$ 34-inch $W$. as the base of the PR stack. Neoprene sheeting $1 / 16$ inches thick was placed underneath the bottom PR bar. The PR is held against the TA by two pieces of 1.5 inches L-channel bolted to the PR support block at the bottom, and bolted at the top to the upper horizontals of the calorimeter frame. The PR stack vertical center is $400 \mathrm{~mm}$ above the top of the deck plate. The TA stack vertical center is $368 \mathrm{~mm}$ above the top of the deck plate.

\footnotetext{
${ }^{3}$ This value was determined from physics data, by comparing PR signals for hits in coincidence with single TA blocks. During the tests of the detectors before E155, a measurement of the attenuation length was made using the cosmic ray trigger; this technique found an attenuation length of about 0.6 meter, but had several problems. Analysis using data from E155X has found attenuation length of about one meter. consistent with the E155 determination.
} 


\subsubsection{Installation Details}

The spectrometer design called for the calorimeter to be centered about $11.5 \mathrm{~cm}$ below the beam height, which is about $207 \mathrm{~cm}$ above the ESA floor. Since the center of the preradiator is about $54 \mathrm{~cm}$ from the bottom of the assembled calorimeter. a stand was constructed to elevate the calorimeter by $36.8 \mathrm{~cm}$, with the remainder of the elevation to be accomplished by placing shims under the feet of the stand. Figure 3.7 shows the completed calorimeter and stand.

The stand for the calorimeter is 14.5 inches $(36.8 \mathrm{~cm})$ tall. constructed of 6 inch $\times 6$-inch aluminium I-beam. The legs are shimmed up about 0.6 inches from the floor. The shimming was also done in such a way as that the deck plate is level both sideways and front-to-back.

In the final installed position, the center of the preradiator is located at $Y^{-}=$ $-114.9 \mathrm{~cm}$ and $Z=1743.3 \mathrm{~cm}$. Table 3.4 shows the locations of each TA and PR block. The $Z$ position was determined by referring to layout marks on the floor: no survey of the $Z$ location of the calorimeter was done before E1.5.5. There is a discrepancy between these positions and the survey done after E15.5X. The PR $Z$ location. based on the post-E155X surveys, is $1748.0 \mathrm{~cm}$. The impact of this shift on the E15.5X analysis, and hence the E15.5 analysis, will be discussed in Appendix A.

\section{Shielding Walls}

The photomultiplier tubes were protected from background particles by three lead shield walls. The first two were in front of the PR photomultiplier tubes to shield them from particles coming through the spectrometer aperture. The third wall is on the side of the shower counter toward the beam pipe. shielding the stack from particles coming through the spectrometer wall. All three walls are one brick thick (4-inch), which corresponds to a thickness of about 18 radiation lengths. Figures 


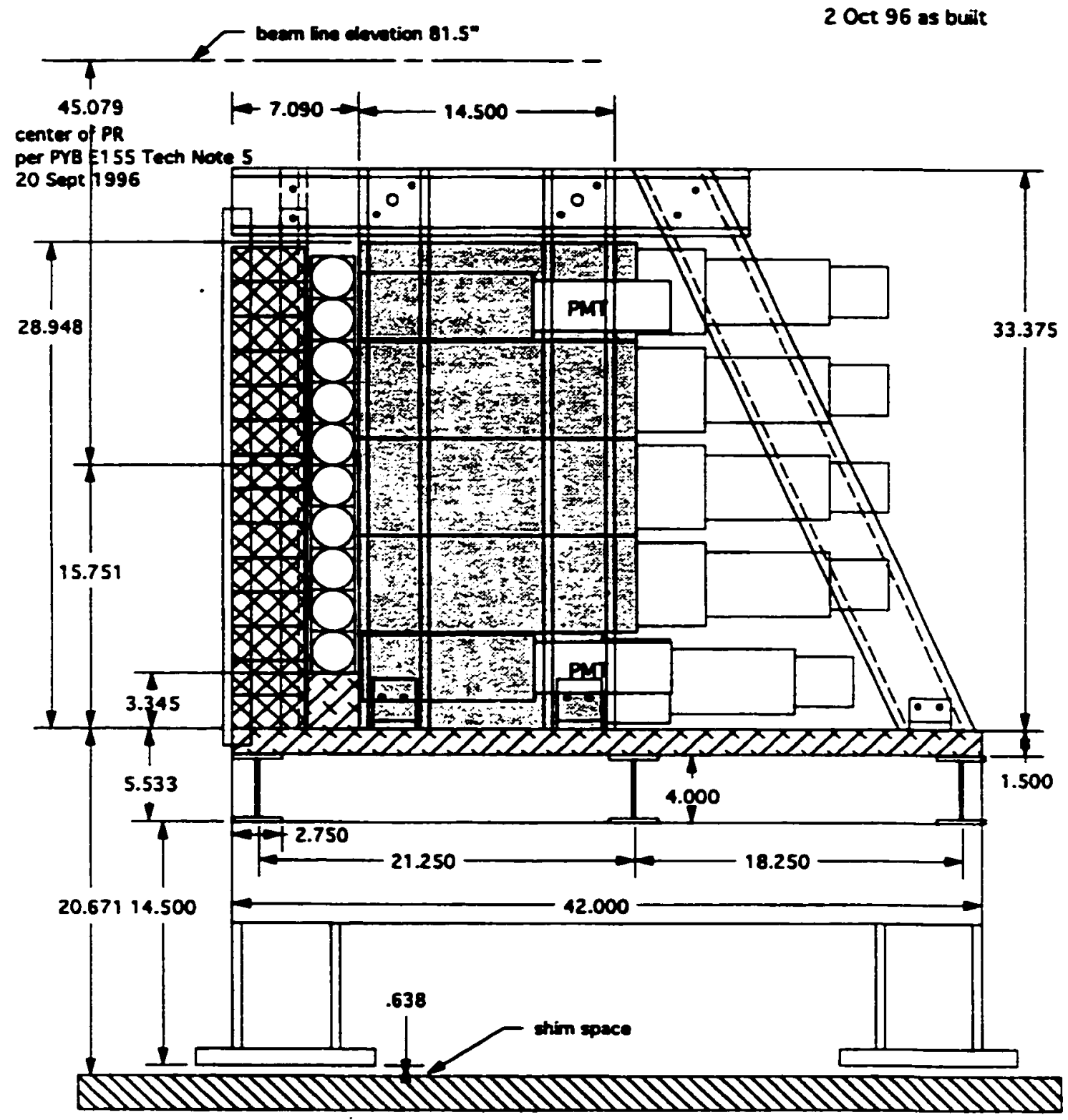

Figure 3.7: Side view of the $10.5^{\circ}$ shower calorimeter assembly and stand. All dimensions are in inches. 


\begin{tabular}{|c|c|c|c|c|c|c|}
\hline TA Column & TA Row & $X_{\text {center }}$ & $\Delta x$ & $Y_{\text {enter }}$ & $\Delta Y$ & $Z$ \\
\hline 1 & 1 & -346.0 & 105.0 & -1452.5 & 104.0 & 17515.0 \\
\hline 2 & 1 & -220.0 & 148.0 & -1473.5 & 146.0 & 17539.0 \\
\hline 3 & 1 & -74.0 & 144.0 & -1474.5 & 146.0 & 17539.0 \\
\hline 4 & 1 & 73.0 & 147.0 & -1473.5 & 144.0 & 17539.0 \\
\hline 5 & 1 & 219.0 & 147.0 & -1473.5 & 146.0 & 17539.0 \\
\hline 6 & 1 & 346.0 & 10.5 .0 & -1452.5 & 104.0 & 17515.0 \\
\hline 1 & 2 & -368.0 & 14.5 .0 & -1326.5 & 147.0 & 17539.0 \\
\hline 2 & 2 & -222.0 & 149.0 & -1327.5 & 147.0 & 17539.0 \\
\hline 3 & 2 & -74.0 & 146.0 & -1327.5 & 147.0 & 17539.0 \\
\hline 4 & 2 & 72.0 & 147.0 & -1327.5 & $1+7.0$ & 17539.0 \\
\hline 5 & 2 & 220.0 & 148.0 & -1326.5 & 147.0 & 17539.0 \\
\hline 6 & 2 & 368.0 & 147.0 & -1327.5 & 148.0 & 17539.0 \\
\hline 1 & 3 & -366.0 & 149.0 & -1180.5 & 146.0 & 17539.0 \\
\hline 2 & 3 & -220.0 & 143.0 & -1180.5 & 147.0 & 17539.0 \\
\hline 3 & 3 & -74.0 & 147.0 & -1180.5 & 147.0 & 17539.0 \\
\hline 4 & 3 & 73.0 & 146.0 & -1180.5 & 146.0 & 17539.0 \\
\hline 5 & 3 & 221.0 & 149.0 & -1180.5 & 147.0 & 17539.0 \\
\hline 6 & 3 & 368.0 & 146.0 & -1180.5 & 146.0 & 17539.0 \\
\hline 1 & 4 & -367.0 & 146.0 & -1034.5 & 147.0 & 17539.0 \\
\hline 2 & 4 & -221.0 & 146.0 & -103.5 .5 & 145.0 & 17539.0 \\
\hline 3 & 4 & -75.0 & 146.0 & -1034.5 & 147.0 & 17539.0 \\
\hline 4 & 4 & 72.0 & 149.0 & -1034.5 & 148.0 & 17539.0 \\
\hline 5 & 4 & 221.0 & 148.0 & -1033.5 & 147.0 & 17539.0 \\
\hline 6 & 4 & 368.0 & 146.0 & -1034.5 & 147.0 & 17539.0 \\
\hline 1 & 5 & -345.0 & 105.0 & -908.5 & 105.0 & 17.515 .0 \\
\hline 2 & 5 & -220.0 & 146.0 & -889.5 & 147.0 & 17539.0 \\
\hline 3 & 5 & -74.0 & 147.0 & -886.5 & 147.0 & 17539.0 \\
\hline 4 & 5 & 74.0 & 147.0 & -887.5 & 147.0 & 17539.0 \\
\hline 5 & 5 & 221.0 & 148.0 & -88.5 .5 & 148.0 & 17539.0 \\
\hline 6 & 5 & 368.0 & 146.0 & -887.5 & $1+7.0$ & 17539.0 \\
\hline \multicolumn{2}{|c|}{ PR Bar } & $\boldsymbol{X}_{\text {center }}$ & $\Delta x$ & $Y_{\text {center }}$ & $\Delta Y$ & $Z$ \\
\hline \multicolumn{2}{|c|}{2} & 0.0 & 750.0 & -1430.0 & 61.0 & 17433.0 \\
\hline \multicolumn{2}{|l|}{2} & 0.0 & 750.0 & -1367.5 & 61.0 & $17+33.0$ \\
\hline \multicolumn{2}{|l|}{3} & 0.0 & 750.0 & $-130.5 . i$ & 62.0 & $17+33.0$ \\
\hline \multicolumn{2}{|l|}{4} & 0.0 & 750.0 & -1244.0 & 61.0 & 17433.0 \\
\hline \multicolumn{2}{|l|}{5} & 0.0 & 750.0 & -1181.0 & 61.0 & 17433.0 \\
\hline \multicolumn{2}{|l|}{6} & 0.0 & 750.0 & -1118.7 & 61.0 & $17+33.0$ \\
\hline \multicolumn{2}{|l|}{7} & 0.0 & 750.0 & -1056.2 & 62.0 & 17433.0 \\
\hline \multicolumn{2}{|l|}{8} & 0.0 & 750.0 & -994.0 & 60.0 & $17+33.0$ \\
\hline \multicolumn{2}{|l|}{9} & 0.0 & 750.0 & -931.2 & 62.0 & 17433.0 \\
\hline \multicolumn{2}{|l|}{10} & 0.0 & 750.0 & -868.2 & 63.0 & $17+33.0$ \\
\hline
\end{tabular}

Table 3.4: Locations of the $10.5^{\circ}$ shower calorimeter blocks within the spectrometer coordinate system. All measurements are in $\mathrm{mm}$. 


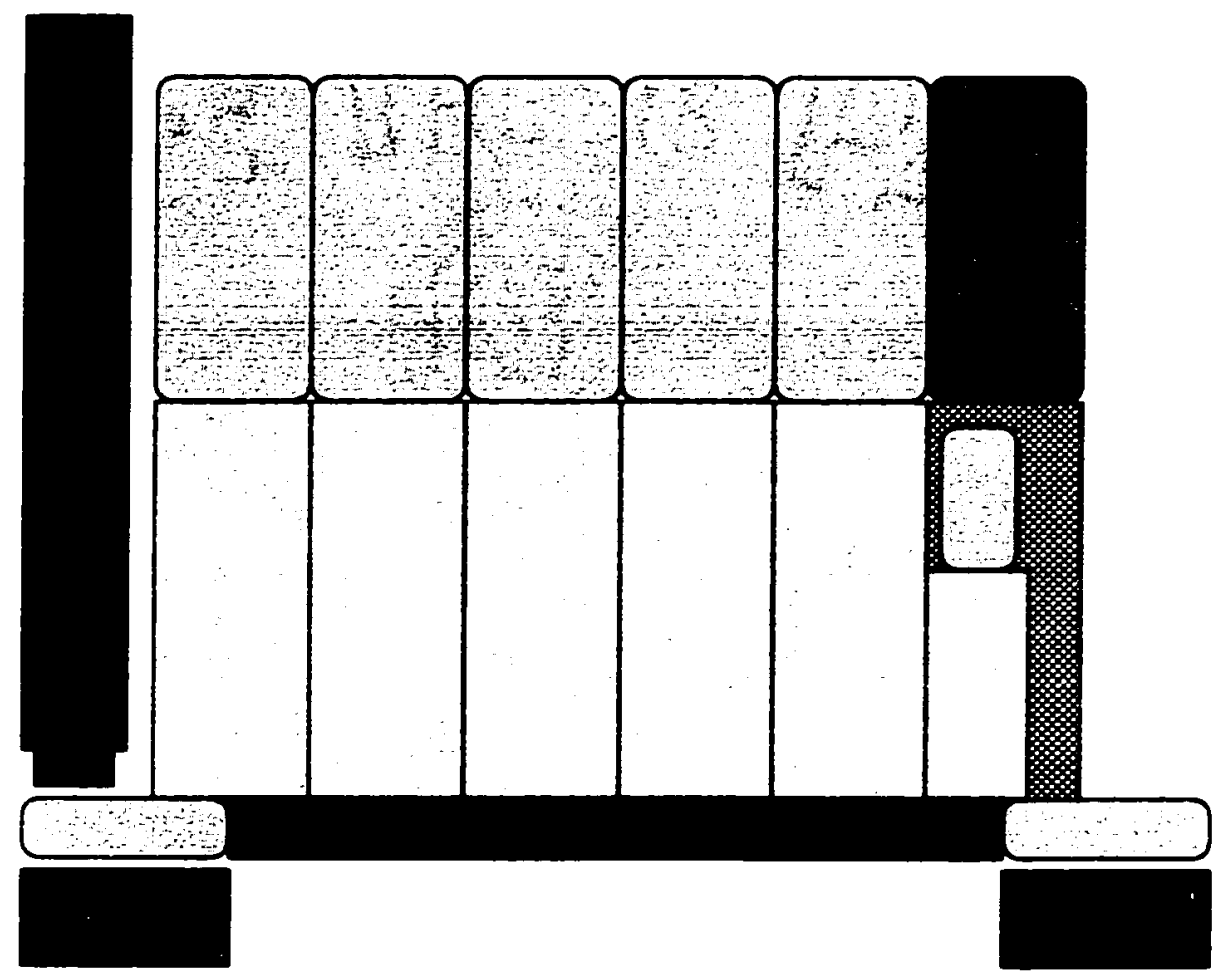

PMT

Total Absorber Block

Preradiator Bar

\section{4th Row PMT}

4th Row TA Block

\section{Lead Brick}

Figure 3.8: Diagram of the TA and PR stacks with lead shield walls, looking from above; the front of the calorimeter is at the bottom of the diagram. The lead glass blocks and PMTs of the top row of the TA stack are shown. The lead glass and PMT of the fourth row block underneath the small TA block are also shown. The support frames of the calorimeter bars and lead shield walls are not shown. The lead bricks in the first column of the side wall are cut to fit into their support frame. 
3.7 and 3.8 show the position of the lead shield walls with respect to the lead glass.

\subsubsection{Calorimeter Calibration and Checkout Before E1.5.5}

\section{Plateauing at Cal-Tech}

The calorimeter was assembled and tested at the California Institute of Technology during the summer of 1996. During the construction of the calorimeter. each completed block was tested using the LED pulser input port. and a high voltage plateau curve was determined using the LED input as the signal. The TA blocks were stacked roughly by plateau voltages, so that neighboring blocks had similar plateau voltages.

PR tubes were plateaued, and high voltages chosen at $2 / 3$ along the plateau. After the bars had been assembled and wrapped. the high voltage was left connected to the bars for several weeks, and the noise signals observed periodically through this time. The bars were stacked in order of increasing noise. with the lowest noise bars at the bottom of the stack. The reason for putting the bars with the lowest noise rate at the bottom was that those bars have the highest particle rate: a high noise rate could contribute to accidental coincidence between the PR and the TA.

\section{Cosmic Ray Testing with the MCA}

After the calorimeter had been moved to SLAC in September of 1996. two scintillator paddles about 6 -inch square were placed above (resting on the TA stack) and below (resting on the floor without the SHW stand installed) the PR stack to form a cosmic ray trigger. Using this cosmic ray trigger. the gains were adjusted to try to match the comic ray signal in all the bars to a value of 6.5 channels above pedestal in a NIM qVt MCA module. This seemed to give a signal height of about $50 \mathrm{mV}$, and it was thought that the pion signal would also be about $50 \mathrm{mV}$.

A similar cosmic ray trigger was used to gain match the TA blocks. 


\section{LED System}

The LED test system utilized two blue LEDs driven by a negative-going pulse of peak-height $-4.38 \mathrm{~V}$. The driving pulse was provided by the same NIKHEF pulser used by the Cherenkov LED test system [110], with a $5.4 \mathrm{db}$ attenuator. passive splitter. and a $1 \mathrm{db}$ attenuator. The pulse width was about $10 \mathrm{~ns}$. There were three layers of plastic over the TA LED, and 4 layers over the PR LED. 


\section{CHAPTER 4 \\ DATA COLLECTION: E155 \& T418}

E1.55 collected data primarily with longitudinal target polarization. but with a short period of transverse target alignment at the end of the two month running period. In addition to the E15.5 experimental run. a test beam experiment. T418. was conducted at SLAC in December of 1998 to provide calibration information for the $2.75^{\circ}$ and $10.5^{\circ}$ spectrometers.

The E1.5.5 checkout running ( $30 \mathrm{~Hz}$ beam pulse rate) period began on 12 February 1997. The first beam pulses entered ESA at about 13:40. The first data with beam written to tape was in Run 40. logged to tape QC1890. The E1.5.5 data collection (120 Hz beam pulse rate) run began on $1 \mathrm{March} 199 \pi$. The first run using the $120 \mathrm{~Hz}$ trigger rate was Run 748. a pedestal run taken at 22:55. The first high rate beam logged was Run 759. written to tape QC:2029. The final beam was logged to tape QC.3896 as Run 4384, which ended at 23:32 on 30 April 1997. A few calibration runs were taken subsequently. bringing the final run number to 4388 . During production running, a normal data collection run took about a half hour to complete, and consisted of about 150000 spills for the $\mathrm{NH}_{3}$ target. or about 180000 spills for the LiD target. A total of 4142 runs were logged during the active running period of E155, filling 2008 1-GB data tapes [111]. Of these runs. the first several hundred (through about Run 800 or 900 ) were not used in the physics analysis due to unstable beam or spectrometer conditions. Of the remaining 3300 runs, many were various forms of calibration, such as Møller runs, LED or pedestal runs. A total of 1663 runs contained electron scattering data in at least one spectrometer. 


\begin{tabular}{|c|c|c|c|c|}
\hline Run Condition & Target & $2.75^{\circ}$ & $5.5^{\circ}$ & $10.5^{\circ}$ \\
\hline$A_{\text {|| }}$, electron & $\mathrm{NH}_{3}$ & 497 & .542 & $52 \pi$ \\
\hline$A_{\|}$. electron & $\mathrm{LiD}$ & $4 \pi 1$ & 470 & .51 \\
\hline$A_{\mid l}$, positron & $\mathrm{NH}_{3}$ & .59 & 38 & 51 \\
\hline$A_{\|,}$positron & LiD & 110 & 142 & 59 \\
\hline$A_{\perp}$, electron & $\mathrm{NH}_{3}$ & 79 & 82 & $8: 3$ \\
\hline$A_{\perp}$, electron & $\mathrm{LiD}$ & 196 & 209 & 204 \\
\hline$A_{\perp}$, positron & $\mathrm{NH}_{3}$ & 5 & 5 & 5 \\
\hline$A_{1}$, positron & LiD & 8 & 8 & 8 \\
\hline
\end{tabular}

Table 4.1: Number of runs used in the physics analysis for each spectrometer for different spectrometer polarities, target orientations, and target materials.

and were used to produce the physics asymmetries as follows: $591 . \mathcal{A}_{\|}^{p}$ runs, $76.5 . \mathcal{A}_{\|}^{d}$ runs. $88 A_{\perp}^{p}$ runs. and $219 A_{\perp}^{d}$ runs. Not included in these counts are the number of positron runs collected (see Section 4.1.3).

The T418 test run began on 25 November 1997 . The first run written to tape was 5090, logged to QC3900 on 3 December 1997. The last run of T418 was 5.52:3. logged to tape QC.3958 at 00:40 on 18 December 1997. Of these 434 runs. 343 were logged to tape. with the remainder used to determine the beam tuning using the online histograms. There were 1.5 beam position runs. $412.75^{\circ}$ spectrometer runs, and $16510.5^{\circ}$ spectrometer runs. Among the other runs. 38 were discarded due to unknown or inconsistent conditions, and 84 were electronics calibration runs (pedestal. LED test. and toroid runs; see Section 4.1.6).

\subsection{E155: Longitudinal \& Transverse Asymmetry Measurement}

\subsubsection{E155 Longitudinal Asymmetry}

The beam energy for the E15.5 longitudinal target polarization running was $48.3 \mathrm{GeV}$. About 110 million beam spills were acquired for the LiD target, and about 88 million beam spills for the $\mathrm{NH}_{3}$ target. 


\subsubsection{E1.55 Transverse Asymmetry}

During the E15.5 transverse target polarization running the beam energy was $38.8 \mathrm{GeV}$. The E15.5 transverse data collection started on April 24 and ended on 30 April. About 32 million beam spills were acquired for the LiD target, and about 12 million beam spills for the $\mathrm{NH}_{3}$ target.

\subsubsection{Positron Runs}

In order to correct the measured asymmetries for contamination by particles which are not deep inelastically scattered electrons. some data were collected in 'positron' mode. In positron running, the polarity of the spectrometer magnets was reversed. so that positively charged particles were accepted by the spectrometers instead of the negatively charged particles. By measuring the rate and asymmetry of positron events, the rate and asymmetry during the electron running may be corrected for electrons which are produced by charge symmetric processes. Section 5.6.2 describes this correction technique from the perspective of the analysis.

To minimize the overall uncertainties in the measured asymmetries. the ratio of data collected in positron mode to data collected in electron mode must be equal to the square root of the fractional positron rate [it]. This rate depends upon scattering angle and energy, and so must be optimized for each spectrometer.

\subsubsection{Solid and Hole Target Runs}

Runs using an unpolarized solid target provided a consistent target density with which to compare the rates during polarized target running. In this manner the packing fraction of the polarized targets could be determined. as a function of time. The three types of unpolarized targets were beryllium. carbon, and the "hole" target, which actually gives the scattering from the liquid helium bath. For each target stick, the "dummy" target contains the solid target which is a close match 
in mass-thickness and radiation lengths to the polarized material in the stick (see Section 2.3.2), and is held within a cell identical in construction to the polarized target cells. The "small" target only contains the solid target material. and was chosen to match the polarized material not used in the target stick.

About 140 runs used a beryllium target and about 100 runs used a carbon target, with the majority of those being the large -dummy" targets. About 15 runs used the hole target.

\subsubsection{Target Anneals \&: Polarization}

When the target polarization dropped below some threshold due to radiation damage, the target was annealed. Thereafter, a thermal equilibration (TE) polarization measurement was made for calibration purposes. and the target repolarized.

As the target is irradiated during the experiment, additional paramagnetic centers are produced in the target. The increased density of paramagnetic centers allows depolarizing transitions to occur. Annealing the target is done to reduce the number of paramagnetic centers in the target material.

For the ammonia target, annealing was required after every 10-16 hours of beam exposure (total dose on target of about 10-16 peta-electrons). The material temperature is increased to about $80 \mathrm{~K}$ for half an hour [112]. Repolarization of the proton target took about 20-30 minutes [111].

For the LiD target material. annealing was required after an incident radiation dose of $10 \times 10^{16} \mathrm{e}^{-} / \mathrm{cm}^{2}$. The material was heated to $18.5 \mathrm{~K}$. and held at that temperature for about 20 minutes before being cooled and repolarized. Polarization for the LiD took between 50-100 minutes depending upon dose [73]. 


\subsubsection{Calibration Runs: Pedestal. LED. Toroid}

About three times per day during E15.5. a set of electronics calibrations runs were done. This included a pedestal run (PED). an LED calibration run (LED). and a toroid mini-calibration run (TOR). These calibration runs were done with the beam suppressed, so that the electronics registered no events related to the beam.

The pedestal runs are used to get pedestal levels for the ADCs and FADCs. The ADCs used in the $2.75^{\circ}$ and $5.5^{\circ}$ spectrometers have a pedestal suppression feature: the $A D C$ pedestals measured during the pedestal runs are downloaded to the module where they are subtracted from the ADC values during normal running and only the information from channels above pedestal are recorded. The $10.5^{\circ}$ ADCs do not have that feature. The $10.5^{\circ} \mathrm{ADCs}$ are recorded to a common block (and the data-stream) where they are accessible to the analysis.

The LED test runs are used to check for gain drifts in the C'herenkov tanks and the $10.5^{\circ}$ shower calorimeter. LEDs coupled to the detectors are fired by a pulser during the normal electronics gate started by the accelerator trigger signal. The electronics modules are read out just as in a normal data collection run. The LED response is (relatively) constant over time. and can be used to monitor and correct for gain drifts in the detectors.

Toroid calibration runs were made periodically, as described in Section 2.2.1.

\section{1.i Møller Runs}

The single arm and double arm Møller polarimeters measured the beam polarization. A total of 329 individual Moller runs were done throughout the experiment. During the data collection, a group of Møller runs was done about every three or four days. At the beginning of the longitudinal and transverse running periods. energy scans were done to calibrate the flip coil value, and verify that the beam energy was set to deliver the maximum polarization to ESA. An additional ten sessions of 
Moller running were done during the checkout period.

\subsection{T418: Calibration Test Beam}

SLAC test beam experiment T418 occurred in December 1997. with the purpose of using a low-intensity parasitic electron beam to calibrate the $2.75^{\circ}$ and $10.5^{\circ}$ spectrometers. A few electrons per spill (the number of electrons was generally between 0.1 and 2.0) were delivered to ESA, and directed into either the $2.75^{\circ}$ or $10.5^{\circ}$ spectrometer aperture by a combination of two $18 \mathrm{D} 72$ dipole magnets and the chicane magnets. Since the electrons entering the spectrometer during T418 were not accompanied by a large background of hadrons, the response of the shower calorimeter and tracking systems to a clean electron could be determined and used to calibrate the E1.5.5 analysis.

I was responsible for producing the run plan for the $10.5^{\circ}$ spectrometer calibration. Seven different electron energy settings were scanned over the spectrometer aperture. with the spectrometer magnets at their normal setting of a central momentum of $11.7 \mathrm{GeV} / c$, and at lowered spectrometer momentum settings to allow delivery of electrons with three different energies into each TA block.

\subsubsection{Beam Production and Delivery}

The beam used for T418 was produced parasitically during operation of the SLAC Linear Collider (SLC). A series of eight collimators in the last three accelerator sectors scraped away a few percent of the SLC electron and positron beam pulses. The electrons or positrons which hit the collimators underwent bremsstrahlung. producing high energy photons, some of which traveled along the beam path. A thin radiator was placed in the beam pipe beyond the SLC splitting magnet to convert these photons into $e^{+} e^{-}$pairs. Some of the electrons entered the A-Line. By adjusting the momentum setting of the A-Line magnets, the momentum of the electrons 


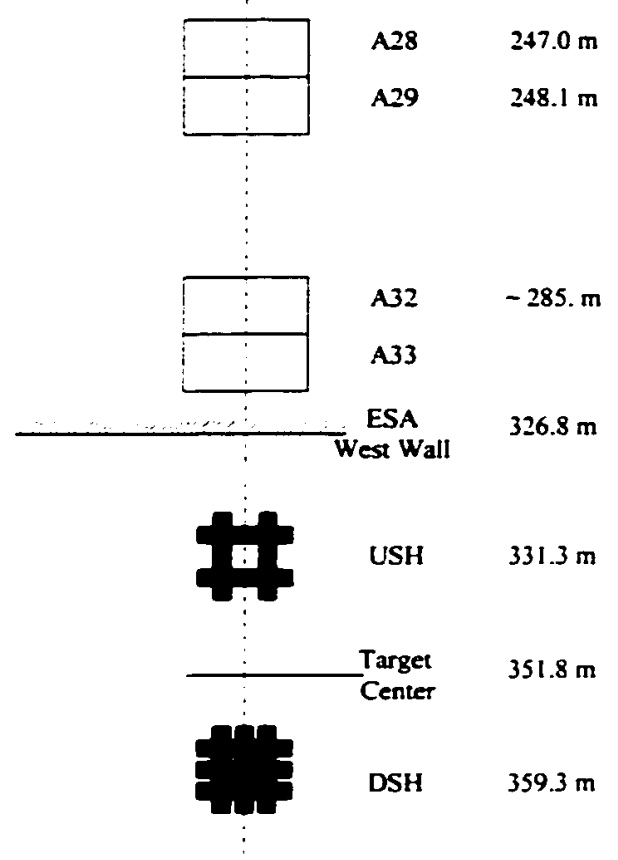

Figure 4.1: Diagram of steering magnet and beam hodoscope locations in the BSY $Z$ reference system. The distances are measured along the beam line starting from the BSY reference point. The steering magnets are dipole corrector magnets: A28 and A32 steer horizontally, and A29 and A33 steer vertically. The LSH and DSH are respectively the up-stream and down-stream beam hodoscopes.

reaching ESA could be changed, and by adjusting the opening of the SL10 slits both the momentum spread of the resulting electrons and the total number of electrons entering ESA could be modified. By this process, a few electrons of definite energy were delivered to the ESA for each SLC beam crossing. The electrons appeared in two bunches separated by about 59 ns, corresponding to the SLC electron and positron pulses [113].

Steering of this low intensity beam within ESA was under the control of the experimenters. A set of two "beam hodoscopes" was used to determine the position and angle of the beam within ESA, diagramed in Figure 4.1. The upstream beam hodoscope consisted of four 1-inch scintillator fingers framing the beam path near the Møller magnet. The downstream beam hodoscope had six 1-inch wide fingers. three horizontal and three vertical, so that the central finger of each set was centered 
on the beam axis. With the chicane and bend dipoles off. the beam was centered through both sets of beam hodoscopes.

\subsubsection{Mapping the Spectrometer Acceptances}

Once the electron beam was centered in ESA, a pair of two 1SDi2 magnets were used to provide $\theta$ deflection about the target center. and the chicane magnets provided $\phi$ deflection. In combination, the bend and chicane magnets delivered electrons of a known energy to various points across the angular acceptance of the $2.75^{\circ}$ and $10.5^{\circ}$ spectrometers.

Most of the data runs were "sweeps". in which the $\theta$ and 0 deflections were individually stepped across the spectrometer acceptance. Thus each data run contains data from a grid of angular locations. At least 500 events (sometimes several times that many) were collected for each grid location.

Data were also taken for spectrometer magnet settings with lower current than normal. This resulted in the electrons being steered into a higher lead glass block than usual, thus allowing several energy calibration points in each block.

For the $10.5^{\circ}$ spectrometer calibration. seven different energy settings were used: 6.0 GeV. T.0 GeV, 8.5 GeV. 9.5 GeV, 11.0 GeV, 13.0 GeV. and 14.5 GeV. A total of fourteen different spectrometer magnet configurations were used. with the magnet currents ranging from their standard setting down to 0.35 times the standard setting. Table 4.2 shows the spectrometer magnet settings used for each beam energy set ting. Also shown are the "grid patterns" used in the sweeps of that energy and momentum setting.

The grid patterns were a listing of pairs of current setpoints for the $\phi$ and $\theta$ magnets to deliver the electron beam to specific locations across the spectrometer acceptance. For most of the patterns (a-g), the particle trajectories were mapped through a model of the spectrometer magnets to determine the $\dot{\phi}$ and $\theta$ deflections 


\begin{tabular}{cccl} 
Beam Energy & B5 Setting & Q2/Q3 Setting & Grid Patterns \\
$6.0 \mathrm{GeV}$ & 04.09 & 04.68 & a. e. f. g \\
& 05.85 & 05.85 & h. i \\
& 06.43 & 04.68 & a. c. d. e. f. g \\
& 08.19 & 04.68 & a. e. f. g \\
\hline $7.0 \mathrm{GeV}$ & 04.68 & 04.68 & a \\
& 07.02 & 07.02 & a \\
& 10.53 & 08.19 & a \\
& 11.70 & 11.70 & a \\
\hline $8.5 \mathrm{GeV}$ & 07.02 & 04.68 & a \\
& 11.11 & 05.85 & a \\
& 11.70 & 11.70 & a. h \\
\hline $9.5 \mathrm{GeV}$ & 07.02 & 04.68 & a \\
& 11.70 & 04.68 & a \\
& 11.70 & 11.70 & a. b. c \\
\hline $11.0 \mathrm{GeV}$ & 07.02 & 08.19 & a.f \\
& 11.70 & 08.19 & a.f \\
& 11.70 & 11.70 & a \\
\hline $13.0 \mathrm{GeV}$ & 10.53 & 08.19 & a \\
& 11.70 & 11.70 & a. c. h. i \\
\hline $14.5 \mathrm{GeV}$ & 10.53 & 07.02 & a \\
& 11.70 & 11.70 & a. b. c \\
& & &
\end{tabular}

Table 4.2: Grid patterns used for each set of beam energy and spectrometer magnet settings. The spectrometer magnet settings are listed as the central momenta in $(\mathrm{GeV} / \mathrm{c})^{2}$. Grid patterns are described in the text.

to hit a specific loation on the shower calorimeter. Patterns " $h "$ and "i" were evenly spaced in either $\theta$ or $\phi$ deflection, and were used in combination with the sieve slits to check track reconstruction.

Grid Pattern "a" The standard grid pattern. "a". consisted of 104 points located at: the center of each TA block ( 30 points). the horizontal center of each TA column at the boundary between each TA row ( 24 points). and the vertical center of each PR bar at $X=0, \pm 1.5, \pm 30 \mathrm{~cm}$ (5 points for each of the ten PR bars).

Grid Pattern "b" Grid pattern "b" was a finer grid pattern. containing 404 points. It included all of the points in pattern " $\mathrm{a}$ ". plus: eight points in the interior 
of each TA block at $1 / 4$ block-width intervals in $X$ and $Y$. except for the edge blocks which skip the outer 3 points per block: the horizontal boundary between each $T A$ row at $1 / 4$ block-width intervals, not including the corners. excluding the outer points for edge blocks; and the lower edge of each PR bar at $X=0 . \pm 15 . \pm 30$ $\mathrm{cm}$.

Grid Pattern "c" Grid pattern "c" was an even finer grid pattern. but was more sparse than pattern "b". 282 points were located along one of five lines across the shower calorimeter face: 51 points along the $X$ of TA column 2 starting at the center of PR bar 2 and moving up in steps of $0.75 \mathrm{~cm}: 60$ points along each of the $Y$ of PR bars 2. 3 . and 4 starting at the $X$ of TA column 2 and ending at TA column 5 , in steps of $0.75 \mathrm{~cm}$; and 51 points along the $X$ of column 5 starting at the center of PR bar 2 and moving up in steps of $0.75 \mathrm{~cm}$.

Grid Pattern "d" This is a subset of pattern "b". centered on TA row 3. column 2. This pattern was used to scan across that block during the period in which its $A D C$ input signal was used as the input to a FADC to investigate the multiple pulse structure of the shower calorimeter.

Grid Pattern "e" This pattern is very coarse. consisting of just the center of each TA block, and the center of each PR bar. It was used during the high voltage scans.

Grid Pattern "f " This grid pattern includes the vertical centers of the PR bars at horizontal locations of $X= \pm 12, \pm 24, \pm 36 \mathrm{~cm}$. plus 51 points along $X=$ $0 \mathrm{~cm}$ running from the bottom of the PR stack to the top in steps of $1.25 \mathrm{~cm}$. This pattern was used during the lead passive preradiator tests for E1.55X. 
Grid Pattern "g" This is a subset of pattern " $\mathrm{e}$ ". used for the PR high voltage scan. which just includes the center of each PR bar.

Grid Pattern “h” This pattern is a sweep across the sieve slits along $\theta$ with a spacing between points of $0.5 \mathrm{mrad}$.

Grid Pattern "i" This pattern is a sweep across the sieve slits along $\varnothing$ with a spacing between points of $0.5 \mathrm{mrad}$.

\subsection{3 $10.5^{\circ}$ Spectrometer Goals For T 418}

The primary objective of $\mathrm{T} 418$ was the calibration of the $10.5^{\circ}$ shower calorimeter energy and verification of the momentum reconstruction. Data for six different energies was collected with the spectrometer in its normal setting (a central momentum of $\left.11.7(\mathrm{GeV} / \mathrm{c})^{2}\right)$ allowing the momentum reconstruction to be checked across about half of the momenta range accepted. Also the sieve slit running allowed checks of the angular reconstruction. The momentum reconstruction was found to be accurate within the uncertainties set by the granularity of the system [114]. The energy calibration using the T418 data was not as successful. Shortly before T418. the LED for the test pulser was found to be loosening in its mounting. After resecuring it, the LED signal was sufficiently different to disrupt direct comparison of shower calorimeter gains between E15.5 and T418. The data did allow tests of linearity with high voltage [115] and effects of stray magnetic fields [116]. The ADC saturation levels were investigated, and while direct use of the information was not possible due to the altered LED response, the indication of significant saturation was noted. Also, the use of the FADC to observe the multiple pulsing structure of the TA signal (Section 5.3.2 discusses the problem in more detail) was useful in more clearly understanding the nature of the problem, if not the origin. 
Another objective was to improve the PR reconstruction algorithm. This also did not lead to any results useful for the E15.5 analysis. A similar goal was to use the T418 data to improve the position determination of the TA stack. However the granularity is such that most showers (those within $2 / 3$ of the block width from the block center) are entirely contained within a single block. Only in a small region along the edge does energy sharing occur. so no improvements were possible.

Finally. T41S allowed the opportunity to test modifications to the $10.5^{\circ}$ apparatus in order to improve its performance for the E15.5X run. High voltage scans of the TA [115] and PR bars were useful in optimizing the high voltage settings for E155X. Also tested was a lead passive preradiator: a $1 / 4$ inch (about 1 radiation length) sheet of lead was placed in front of the preradiator. The purpose of the lead sheet was to begin the electromagnetic shower earlier (in the passive lead laver) so as to increase the energy deposited in the active preradiator bars. These tests were promising and encouraged the installation of the lead passive preradiator (or PbPPR) in E1.5.5X. 


\section{CHAPTER 5}

\section{DATA ANALYSIS}

The purpose of the analysis software is to transform the raw ADC and TDC values recorded for the various detector elements into electron scattering rates for each beam helicity and each bin in $x$ and $Q^{2}$. First. particle track events must be recognized from the array of $A D C$ and TDC data. Then these reconstructed tracks must undergo particle identification. to find the electron events. Then energy and scattering angle must be determined for each electron event. For each spectrometer. the number of events within a certain kinematic range is recorded for the two beam helicities.

There are several intermediate stages of data processing within the E1.5.5 analysis framework. In the first stage, the raw data analysis constructs particle tracks through the spectrometer subsystems using the raw $A D C$ and TDC data. At this stage. most of the calibrations have already been done. including the application of timing offsets, energy calibration, detector positions, and slewing corrections. Once the tracking was working reliably, data summary tapes (DST) were produced. The purpose of the DST format was to reduce the time required to perform the physics analysis on a run. The analysis of a raw data tape required about twenty hours on the SLAC batch analysis computers (IBM RS/6000 43P machines running AIX). most of which was consumed by the shower calorimeter and tracking subsystem analyses. By recording the analyzed events in each subsystem spill-by-spill. a considerable savings in re-analysis time was achieved. The re-analysis of a full run DST (corresponding to one raw data tape) required about a half hour. Additionally; the 
DST files required less storage space, being about $1 / 2$ or $1 / 3$ the size of the raw data file. The DST records contain the analyzed data for the Cherenkov and shower calorimeter subsystems, and information on all of the tracks found. The DSTs are re-analyzed using a variety of particle definitions to produce summary files. An example of a simple electron definition would require a track with a high energy shower, a large Cherenkov pulse height. and an $E / P$ ratio in the range of 0.8 to 1.2 . The definitions may also have different requirements for the beam conditions. The summary files, or count files, list the number of events satisfying the definition for each beam helicity state in each $x$ bin. the total beam charge accumulated for each definition in both beam helicity states, and the average value of $x$ and $Q^{2}$ for the $x$ bin. Finally, the data contained in all of the summary files for a particular event definition and target material was collected, and the raw asymmetry was produced by the physics analysis code. The physics analysis routine then applied corrections to the raw asymmetry. in order to produce either $A_{\|}$or $A_{1}$.

\section{$\underline{5.1}$ Raw Data Analysis}

The spill-by-spill unpacking and analysis of the raw data was handled identically for both the online and offline software. The raw data from the beam instrumentation was interpreted to give the beam charge. polarization. and location of the beam spot. The data stream from a spectrometer CAMAC branch consisted of a list of ADC channel-and-value pairs, a list of TDC channel-and-time pairs. and the FADC waveforms from the Cherenkov detectors. These raw data had calibrations applied, then underwent processing by the Cherenkov and shower calorimeter analysis subsystems resulting in lists of Cherenkov and shower calorimeter events. The tracking analysis subsystem took the calibrated hodoscope data, and the lists of Cherenkov and shower calorimeter events, and searched for sets of events with comparable times which align along a straight line through the spectrometer. 


\subsubsection{Beam Analysis}

The beam analysis routine took the raw data from the beam electronics and transformed it into the quantities needed by the spectrometer analysis routines. The most important quantities produced by the beam analysis were: beam charge. polarization state, beam position at the target, beam angle at the target. and beam spot size. Additional information used in the spectrometer analysis were the good and bad spill counter signals. The beam charge was gotten by applying the toroid calibrations. One of the toroids was chosen as the measured value (the first ESA toroid for E1.5), and the other was used as a cross-check. The beam polarization status was provided from the polarized electron source to the data acquisition through four slightly different channels [117]. For the polarization bit to be accepted. the four indicators had to agree [4i]. Additionally. during offline analysis. the polarization state could be predicted by utilizing the algorithm described in Section 2.1 to determine the polarization bit from the preceding spills. The foil array analysis determined the beam spot location and size at the foil array. The traveling wave beam position monitor (TWBPM) provides the beam location before the target. By interpolating between the TWBPM and foil array beam locations. the beam location and angle at the target was determined. An additional indication of the beam location at the target was provided by the raster magnet settings, which determined the location within the raster pattern, but could not monitor any drifting of the center of the raster pattern. The good and bad spill counters were monitored for particularly high or low values which would indicate a mis-steered or low quality beam pulse.

\subsubsection{Spectrometer Data Unpacking and Calibration}

Each TDC hit entered the data stream as three pieces of information. the TDC value, the channel position, and the hit type. The channel position was decoded into 
the channel and module number of the TDC channel, and the raw TDC information was transformed by applying timing offsets and gain corrections. using

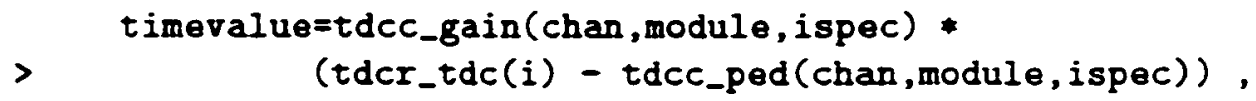

where timevalue is the calibrated time, $t d c c$ gain and $t d c c$ ped are the gain and offset values for the specified TDC module and channel. and tdcr_tdc is the raw TDC value for the hit. The tdcc gain was $1 \mathrm{~ns}$ for all of the E1.5.5 TDC modules. The TDC time offsets were determined for each channel by measuring the time-offlight corrected time difference between hits in the channel and the C'herenkov FADC' hit time. This process will be discussed in more detail for the $10.5^{\circ}$ spectrometer in Section 5.2.3. There was a situation in which some TDC hits were duplicated in the data stream. so the TDC hit arrays are scanned to remove the duplicated hits. In later code, this is done by Zen Szalata's C-code data-stream unpacker.

Since most of the ADCs were zero-suppressed. the ADC data does not undergo any analysis before being used by the detector analysis subroutines. However. the ADC data does exhibit the same repetition as the TDC data under some circumstances. So, the ADC hit arrays are also scanned by the unpacker to remove duplicated hits. The $10.5^{\circ} \mathrm{ADC}$ s were not zero-suppressed. so the pedestals were subtracted during the subsystem analysis, as will be discussed later.

The FADC raw data is composed of up to $\mathrm{cp}_{\text {maxwf }}$ data points (cp_maxwf $=$ 500 in E155), each representing the Cherenkov signal in one time bin. The time bins correspond to approximately one nanosecond. In each time bin. the signal is digitized into an S-bit word. The data points are produced by one of four channels. each of which has an individual pedestal and gain. Figure 5.1 displays a raw Cherenkov waveform in which it is possible to see the variation in pedestal between the channels. The software array storing the waveform is filled using Eq. 5.2, 


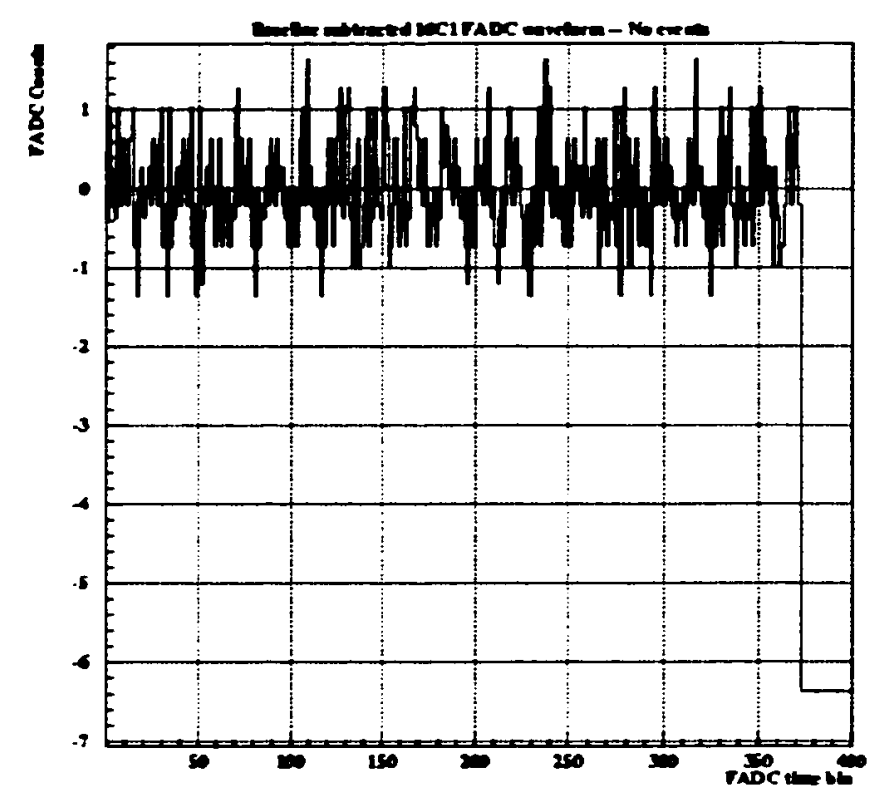

Figure 5.1: The raw $10.5^{\circ}$ Cherenkov FADC waveform in a spill without a Cherenkov hit.

$$
\begin{aligned}
& k=\bmod (j-1,4)+1 \\
& \text { cer_vaveform }(j, \text { itank, ispec })=\text { fadc_ao }(k, \text { itank, ispec })+ \\
& >\quad \operatorname{cer} \_f a d c(j, i t a n k, i s p e c) \text { * fadc_al (k,itank, ispec) }
\end{aligned}
$$

where $k$ is the channel index, $j$ is the time bin. itank and ispec are the tank and spectrometer indices, cerfadc $(j, i t a n k, i s p e c)$ contains the raw $F A D C$ values for the tank, cerwaveform( $j, i t a n k, i s p e c)$ contains the corrected values. and fadc_ao and fadc_al are the pedestal and gain corrections for the kth channel.

\subsubsection{Subsystem Analysis}

The Cherenkov detector analysis used a peak-finding algorithm to find the time at which the voltage reached its peak, the peak voltage and also integrated the charge of each hit within the FADC data. An average Cherenkov peak is shown in Fig. 5.2. During some running periods, the clock signal which controlled the Cherenkov digitization would not be properly read, and the pulse time determined by the FADC would shift by about 4 ns (four time bins). This was monitored by comparing the 


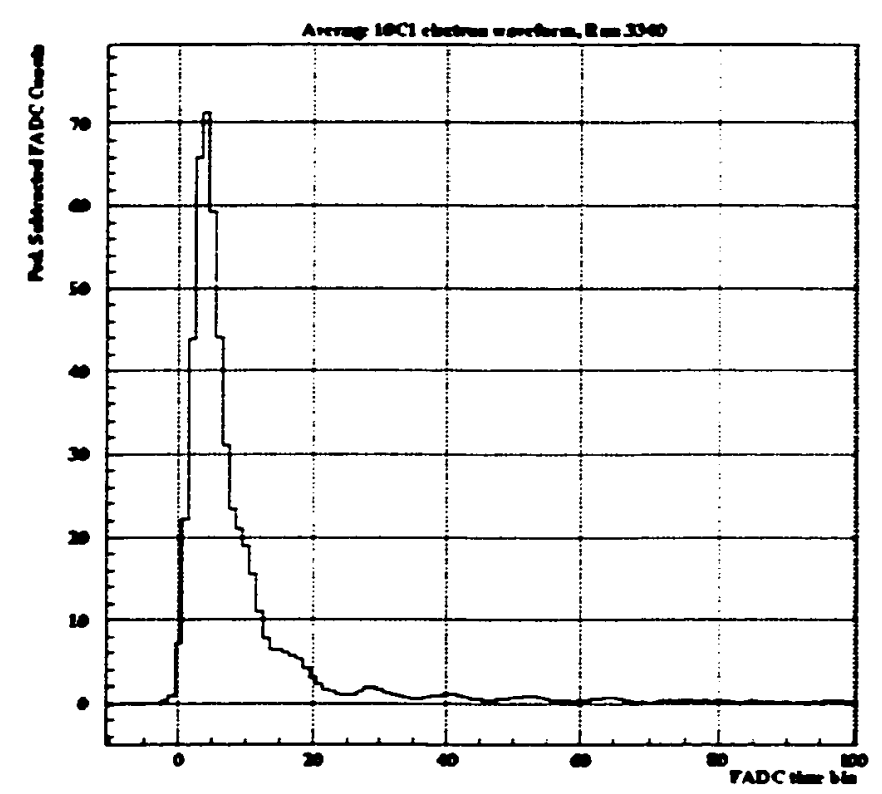

Figure 5.2: Average pedestal-subtracted Cherenkov waveform.

time difference between the FADC times and the TDC times for each spill. and correcting the FADC time by applying a time offset of four bin widths if there was disagreement. When the FADC system failed during part of the experiment. the back-up system, described in Section 2.4.3. was used to find hit times and coarsely bin the peak voltage of each hit. Using either the main analysis or the backup system, the output from the Cherenkov analysis was a data array of Cherenkov hits, each composed of the hit time, peak height, and the integrated charge.

The purpose of the hodoscope subsystem analysis was to prepare an array of timing hits in hodoscope fingers to be used by the tracking analysis. Since the TDC values had been converted to time values in the unpacking routine, the hodoscope analysis just sorted the TDC hits from each channel into the variables for the corresponding hodoscope finger. The hodoscope output array consisted of an array for each finger of the hit times in that finger. Also provided was the geometry 


\begin{tabular}{|cccc|}
\hline Track Type & Cherenkov & Calorimeter & Hodoscope \\
\hline 1 & Yes & Yes & Yes \\
2 & No & Yes & Yes \\
3 & Yes & No & Yes \\
4 & No & No & Yes \\
\hline
\end{tabular}

Table 5.1: Spectrometer subsystems included in each track type.

information describing the location of each finger within the spectrometer.

After the raw shower calorimeter ADC data had their pedestals subtracted and the energy calibrations had been applied. the timing data for each block was used to divide the energy deposited into cells, each with a portion of the total energy. Each timing hit within a lead glass block was identified with a particular fraction of the total energy deposition. Then. a cellular automaton was used to build shower clusters in the calorimeter by matching cells in adjacent blocks [118]. The resulting shower clusters were analyzed to determine the total energy, position. and time of the shower. Since most electron showers are contained within a $3 \times 3$ sub-array of the $2.75^{\circ}$ and $5.5^{\circ}$ calorimeters [109], the block with the largest deposited energy is designated as the central block. Two shower cluster energy values are calculated: $E_{\text {rec }}$ contains the total energy of the cells which were used to build the cluster. and $E_{9}$ contains the sum of the energies of the nine block sub-array centered on the central block. A multi-layer neural network could be used to provide pion-electron particle identification for each shower, but this analysis tool was not used in E1.5.5 despite its success in the previous experiments. The neural network was swamped by overlaps in E15.5, and could not provide a tool for pion rejection. The calorimeter output listing has, for each shower, the time, energy deposited. position and width of the shower, and energy in each of the nine blocks surrounding (and including) the central block.

The tracking analysis used the output arrays from the Cherenkov. hodoscope and calorimeter subsystems to locate particle trajectories within the spectrometer 
hut. Once a particle trajectory was found. a lookup table was used to reconstruct the particle's momentum, scattering angle. and initial scattering location. Four track types were possible, shown in Table 5.1. depending upon which subsystems contribute to the track. Type 1 tracks have hodoscope. Cherenkov and calorimeter hits. A track with only calorimeter and hodoscope hits is of Type 2. Type 3 tracks have only Cherenkov and hodoscope hits. A track with neither Cherenkov nor calorimeter hits is Type 4 .

First, the tracking routine built hodoscope clusters within the forward and rear hodoscope packages. Each hodoscope cluster was composed of fingers which overlap each other in space and have hits at similar times. The hodoscope clusters have $\boldsymbol{X}$, $Y, Z$, and $T$ coordinates. The tracking code then looped through the Cherenkov hits. calorimeter clusters and hodoscope clusters. to find the set of hits which form tracks with the lowest $x^{2}$ in time and position. The timing values from the hodoscope fingers and Cherenkov detectors were corrected for light propagation time within the detectors. Once a track was found, it was mapped back through the magnetic optics to determine the momentum and scattering angle of the particle. The vertical scattering angle. $\dot{\phi}$, and the track momentum. $P$. were reconstructed from $Y_{S H W}$. the vertical location of the track at the calorimeter and $\Delta Y$. the slope of the track in the spectrometer. The values of $X_{\mathrm{SHW}}$, the horizontal position at the calorimeter. and $\Delta . X$, the slope of the track, were used to determine $\theta$. the horizontal scattering angle, and $Z_{0}$, the $Z$ location of the track at the target.

Once $\theta . \phi$ and $P$ had been determined, the $x$ and $Q^{2}$ of the scattering event could be calculated. However, the list of tracks included more than scattered electrons. and an event selection analysis had to be done to determine which ones corresponded to scattered electrons. 


\subsection{Analysis of the $10.5^{\circ}$ Raw Data}

The raw data analysis for the $10.5^{\circ}$ data largely uses the same software routines as the $2.75^{\circ}$ and $5.5^{\circ}$ spectrometer analysis. Most of the differences in the $10.5^{\circ}$ analysis code are related to the shower calorimeter, although there are some differences in the Cherenkov counter and tracking routines as well.

\subsubsection{Modifications to the Tracking Code for the $10.5^{\circ}$ Analysis}

The tracking analysis subsystem required two position measurements in each of $X$ and $Y$ to calculate the values of $X_{\text {SHW }} \Delta . X$. $Y_{\mathrm{SHW}}$, and $\Delta Y$. The $10.5^{\circ}$ spectrometer had only one set of $Y$ hodoscopes at the front of the detector hut. and no $X$ hodoscopes. The second $Y$ point for the tracking was provided by the shower calorimeter. which also yielded a measurement of $X$. A second $X$ coordinate was given by a "fake" hodoscope plane in the analysis software: all tracks were reconstructed through $X=0$ at a $Z$ of 5 meters. This fake hodoscope plane approximately corresponded to the image of the target. The sigmas of the fake plane were $10 \mathrm{~mm}$ in $X$ and $1000 \mathrm{~ns}$ in $T$ : the time of the fake hit was taken to be 0 . The fake plane was not used to generate the $\Delta X$ used for the determination of $\theta$.

The reverse matrix elements for mapping the particle trajectory through the spectrometer optics did not use the same process as for the $2.75^{\circ}$ or $5.5^{\circ}$ spectrometers. Just as in the other spectrometers, $Y_{\mathrm{SHW}}$ and $\Delta Y$ were used to determine 0 and $P$. Then, $P$ and $\boldsymbol{X}_{\text {SHW }}$ were used to determine $\theta$, instead of $X_{\text {SHw }}$ and $\Delta X$.

\section{$5.2 .2 \quad 10.5^{\circ}$ Shower Calorimeter}

The raw data analysis for the total absorber showers also used the same cellular automata clustering analysis as the other spectrometers. The analysis of the preradiator showers used a less sophisticated algorithm, after which the TA and PR clusters were combined. 
The purpose of the preradiator analysis was to find showers contained in one or several bars, determine the $X$ and $Y$ position of the shower on the stack face. determine the time of the shower, and the energy deposition in the preradiator for that shower. First, the routine tried to associate a pair of TDC hits in the two photomultiplier tubes with each other, to form a single shower. The $X$ position of this shower can be determined from either the ratio of charge accumulated in the two photomultiplier tubes, or from the time difference between the TDC times in the two photomultiplier tubes. The determination of $\boldsymbol{X}_{\text {charge }}$ is only good in the case that there is only one shower within the bar during that spill, since if multiple showers occur, the $X_{\text {charge }}$ will be the energy weighted average of each shower's position, as the ADC gate is open for the entire spill. The $\boldsymbol{X}_{\text {time }}$ determination requires that the shower be composed of a hit in each end of the bar, and so is not good for showers which do not fire the discriminator on both of the photomultiplier tubes. For showers with valid $X$ determinations using both techniques. an error weighted average of $X_{\text {charge }}$ and $X_{\text {time }}$ determines the final $X$. The hit times in the two photomultiplier tubes are averaged to give the shower time. The square root of the product of the energy deposition in each end gives the total energy deposition for the shower, which uses the assumption that the light attenuation is exactly exponential, so that as a function of $\boldsymbol{X}$ position.

$$
\begin{aligned}
& E_{1}(X)=E_{0} \mathrm{e}^{-(D+X) / A}=E_{0} \mathcal{D} \mathrm{e}^{-(X / \lambda)} \\
& E_{2}(X)=E_{0} \mathrm{e}^{-(D-X) / A}=E_{0} \mathcal{D} \mathrm{e}^{(X / \lambda)}
\end{aligned}
$$

where $E_{1}$ is the energy signal collected by the tube at $X=-3 i .5 \mathrm{~cm} . E_{2}$ is the energy signal collected by the tube at $X=37.5 \mathrm{~cm}, E_{0}$ is the non-attenuated energy signal. $D=37.5 \mathrm{~cm}$ is the distance from the photomultiplier tube to the bar center, and $\mathcal{D}=\mathrm{e}^{-(D / \lambda)}$ is the attenuation factor due to traversing half the bar length. 
Then $\sqrt{E_{1}(X) E_{2}(X)}$ is independent of $X$,

$$
E_{\text {total }}=\sqrt{E_{1} E_{2}}=E_{0} \mathcal{D}
$$

and the constant factor $\mathcal{D}$ may be incorporated into the energy calibration of the PR bars. For spills in which there are multiple hits in a PR bar. the total energy is divided by the number of hits in the bar to give the energy for each shower.

$$
E_{P R}=\frac{\sqrt{E_{1} E_{2}}}{n_{e v t}} .
$$

where $E_{P R}$ is the energy deposited in the PR for the shower. and $n_{\text {ext }}$ is the number of hits in the bar for this spill. When all of the showers in each bar have a time. position. and energy, showers in adjacent bars are compared in $X$ and $T$ to determine if they could have resulted from the same particle. If the adjacent showers agree in space and time, their energy is added together. and the $X$ and $T$ are averaged to give the $X$ and $T$ of the combined shower. The $Y$ position of these combined showers is the average of the bar $Y$ positions; single bar showers use the $Y$ of the bar.

After the raw ADC and TDC information has been interpreted as a set of showers in the preradiator layer and total absorber layer. the two sets are compared to build shower clusters using both layers. If a PR shower is found which is within a certain range in $X, Y$, and $T$ of a TA shower, a combined cluster is created. If a TA shower does not have a corresponding PR shower. or if a PR shower does not have a TA shower corresponding, then these showers are placed in the final list of clusters. Only calorimeter events which contain TA shower information are used in the tracking analysis.

\subsection{Sectrometer Calibration Projects}

To transform the raw TDC and ADC data into a set of time and energy measurements, the raw data must have calibrations applied. 


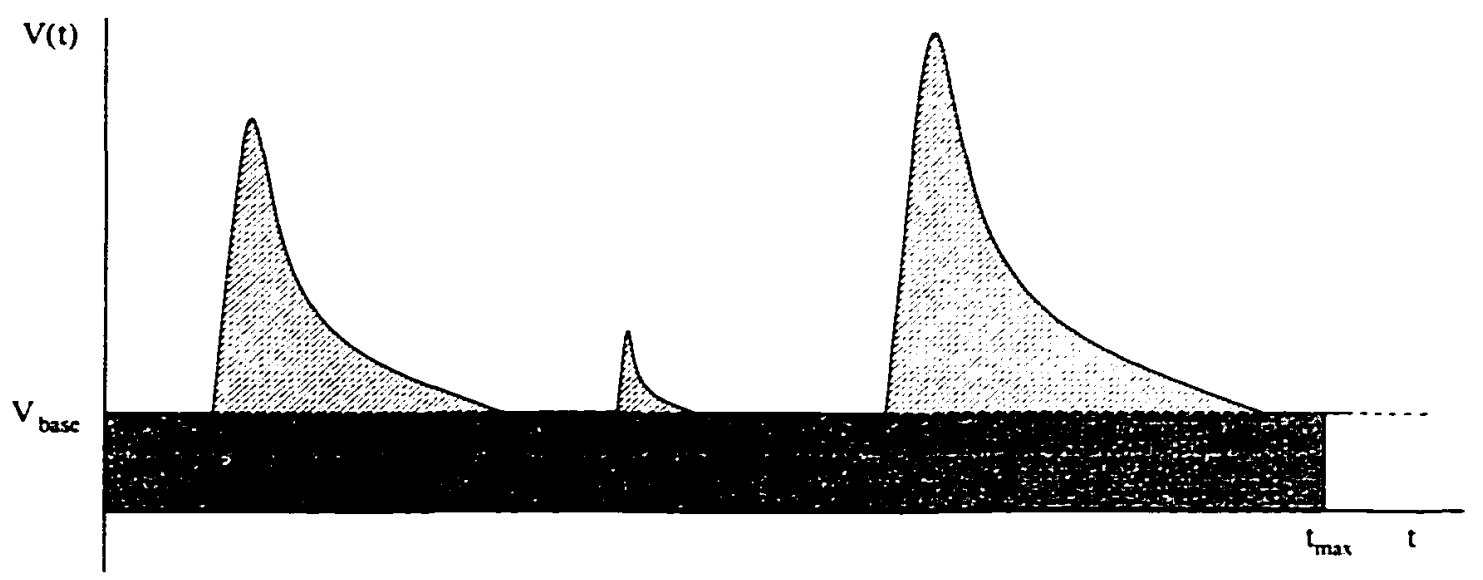

Figure 5.3: Diagram showing the hardware pedestal with three pulses.

\section{ADC Pedestal Correction}

An ADC will have a hardware pedestal due to the difference between the input baseline and the ADC reference voltage. Figure 5.3 depicts the pedestal signal with three pulses. The ADC signal due to the three pulses would be increased by the ADC signal due to the constant baseline voltage. $l_{\text {base. }}$ The ADC values therefore had to be corrected by subtracting the ADC values for a gate in which no pulses occurred.

\section{Timing Offset Calibration}

Since all electron events in the spectrometer have a Cherenkov pulse, the Cherenkov time was used as the reference time for the spectrometer. The time data for the hodoscope and shower calorimeter TDC channels had a timing offset applied. so that a time-of-flight corrected time difference between the channel and the Cherenkov pulse time (Eq. 5.8) would be distributed about zero. Thus the time of a hit in the $i$ th TDC channel, $t_{i}$, is defined as,

$$
t_{i}=(\text { TDC Gain })_{i}\left[(\text { TDC Value })_{i}-\left(t_{\text {offset }}\right)_{i}\right]
$$

where the TDC channel resolution, (TDC Gain) ${ }_{i}$, was 1 ns per TDC channel for all of the E15.5 TDC channels. 
The determination of the timing offsets was done in two stages. In -Timing-1". each Cherenkov hit with a pulse height in the electron range (FADC signal between 40 and 200 counts) was used as the reference event. The time difference for each TDC channel, $\Delta t_{i}$, was calculated using.

$$
\Delta t_{i}=t_{i}-\left(Z_{i}-Z_{\tilde{\mathrm{C}}}\right) / c-t_{\overline{\mathrm{C}}}
$$

where $t_{i}$ is the TDC time. $Z_{i}$ is the $Z$ coordinate of that detector, $Z_{\bar{C}}$ is the $Z$ coordinate of the Cherenkov mirror, and $t_{c}$ is the time of that Cherenkov hit. If the time offset was correct. $\Delta t_{i}$ would be distributed around zero. To correct the time offset, the average of $\Delta t_{i}$ was added to the time offset for that channel. The Timing- 1 analysis was repeated until all the channels had $\Delta t_{i}$ distributions centered within \pm 1 ns of zero, but usually only one iteration was needed.

"Timing-2" utilized the times of tracking hits to correct the time offsets. Since the tracking analysis would not work correctly if the detector elements had wildly different timing, this analysis could not be run until Timing-1 was completed. The time difference for each channel was similar to Eq. 5.8.

$$
\Delta t_{i}=t_{i}-Z_{i} \text { (Ang.Corr.) } / c-t_{\mathrm{TRK}}
$$

where the Ang.Corr. factor corrects the time of flight to the detector for the small distance variation due to the slope of the track. Because the Timing-2 analysis uses the track time, which is based upon the times of the detector hits composing the track, this analysis requires multiple iterations to correct the time offset.

\section{Shower Calorimeter Time Slewing Correction}

If the electronics pulses produced by a detector have about the same pulse height, then only one timing offset is needed, because the discriminator will fire a fixed time after the pulse begins. However, if a detector produces pulses that differ 


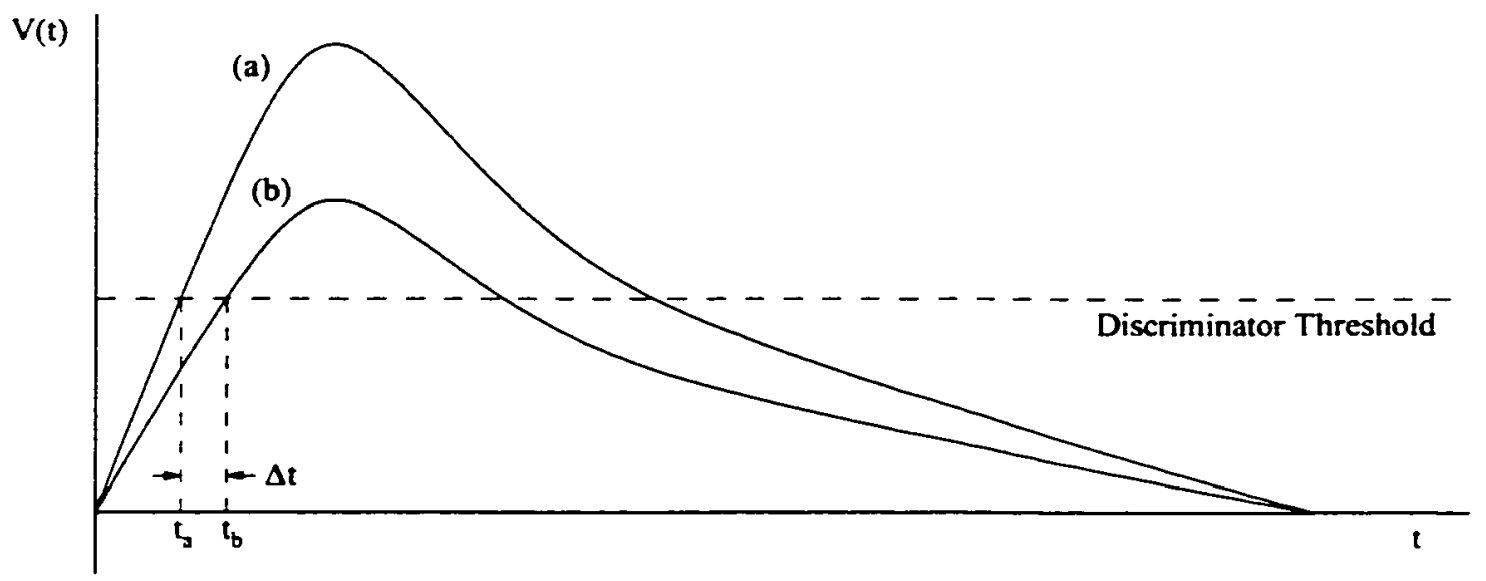

Figure 5.4: Diagram showing time slewing difference between two pulses starting at the same time but having different amplitudes. The small pulse. (b), crosses the discriminator threshold later than the larger pulse. (a), by a time interval. $\mathrm{J}$.

significantly in height, such as the two pulses in Fig. 5.4. the pulses will fire the discriminator at different times after the beginning of the pulse. This pulse height dependence of the timing is called time slewing. In the hodoscopes, since all of the particles have high energy (they are all minimum-ionizing), the pulses are all about the same height. so time slewing does not much affect their timing. However. the shower calorimeter has a very wide range of pulse heights. and time slewing can cause timing variations of several nanoseconds. By performing slewing corrections on the shower pulse times, the time distribution of hits will become narrower, and the coincidence windows may be made narrower.

First Pass Analysis The first pass slewing analysis [119] examined. for each shower block. the relation between the ADC signal and the time difference between the TDC hits and the Cherenkov hits. Only TDC hits which were within $1.5 \mathrm{~ns}$ of a Cherenkov hit with pulse height of at least 25 FADC counts were included in this study. The results were combined into four types of shower blocks: preradiators (PR), small corner total absorber blocks (TAs) on three of the four corners. short TAs along the bottom row, and Long TAs for all other positions. 


\begin{tabular}{rccc} 
Block Type & A & B & C \\
\hline PR & 2.027 & 0.432 & -0.170 \\
Short TAs & 7.552 & -2.142 & 0.165 \\
Long TAs & 5.056 & -1.273 & 0.026 \\
Corner TAs & 48.99 & -13.70 & 0.813
\end{tabular}

Table 5.2: Fit coefficients for Eq. 5.10 for each block type.

\begin{tabular}{rccc} 
Block Type & A & B & C \\
\hline Short TAs & 13.38 & -3.177 & 0.16 .5 \\
Corner TAs & 61.54 & -15.47 & 0.813
\end{tabular}

Table 5.3: Fit coefficients for Eq. 5.10 for the "Short TA" and "Corner TA" block types. using the new ADC ranges. The "PR" and "Long TA" fits in Table 5.2 were not reanalyzed.

As in the timing offset analysis, the time difference for each TDC hit was determined using Eq. 5.8. Ten bins, evenly spaced in $\ln (A D C$ Value), were used to collect histograms of $\Delta t$ for each ADC range for each type of shower block. Runs 3330. 33334 - 33336. and 3340 were analyzed in this way, to form $\Delta t$ distributions as described. Typically, four of the ADC range bins had sufficient statistics to be used in the analysis. Gaussian fits were used to determine the centroid of $\Delta t$ for each $A D C$ bin and block type. A polynomial fit was used to determine the relationship between the bin average of $\ln (A D C$ Value) and the $\Delta t$ cent roid in the bin. We found that each block type displayed a quadratic dependence on $\ln ($ ADC Value). so that the time slewing correction is given as.

$$
t_{\text {corr }}=A+B \times \ln (\mathrm{ADC} \text { Value })+C \times[\ln (\mathrm{ADC} \text { Value })]^{2} .
$$

with the coefficients for each block type shown in Table 5.2. In application. the $t_{\text {corr }}$ value is computed every spill for each block with TDC hits. The $t_{\text {corr }}$ is then subtracted from each hit time in the block.

Slewing Recheck and Second Pass Analysis For the corner TA blocks and the short TA blocks, the first pass slewing analysis was not sensitive enough to the low $\mathrm{ADC}$ value dependence of the time slewing: a timing dependence on the ADC 


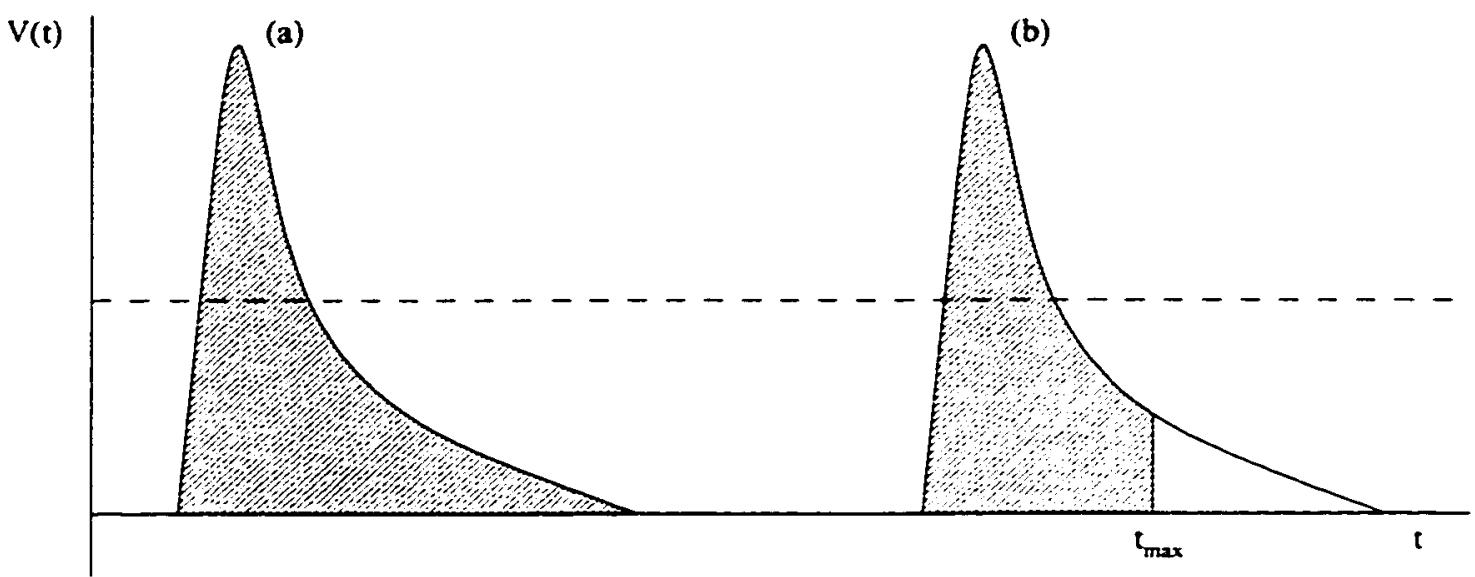

Figure 5.5: Diagram showing the effects of an insufficiently long ADC gate upon the amount of charge integrated by two pulses at different times. The ADC integrates the charge over the time window from $t=0$ to $t=t_{\max }$. Pulse (a) is fully measured. but pulse (b) has part of its charge excluded from the gate. Pulse (b) would therefore be measured as a smaller pulse. even though the pulses are identical.

value was still observable. The slewing corrections for the long TA blocks and the PR bars seemed to adequately describe the ADC dependence of $\Delta t$. In the second slewing analysis. the $\Delta t$ was plotted for each block as a function of the ADC value. From these plots, ranges in $\mathrm{ADC}$ value containing approximately equal numbers of events were chosen, and a Gaussian fit to the $\Delta t$ distribution was made for each of these bins. There were typically nine ranges for which $\Delta t$ fits were made. The mean ADC value in each range was determined, and was used to determine the fit detailed in Eq. 5.10. The new fit coefficients are listed in Table 5.3.

Time-in-spill Analysis In conjunction with the time slewing analysis, the mean $A D C$ value dependence upon the time-in-spill was investigated. Figure 5.5 shows a cartoon picture of how the mean ADC value could decrease for events late in the spill. Similarly, if the pulses have ringing associated with them. the mean $A D C$ value could increase for late events by having less of the ringing included in the ADC gate. The analysis indicated that there was neither a significant or stable dependence on the time-in-spill [119]. 
Cherenkov Peak Height Slewing Analysis The last slewing-associated check was to verify that the Cherenkov pulse height did not affect the $\Delta t$ between the Cherenkov pulse time and the hit time in the other detectors. The Cherenkov analysis uses a pulse shape fitting technique to determine the time of the beginning of the pulse, so there should be no slewing corrections to the detector hit times due to the Cherenkov pulse height. There was no noticeable slewing in the shower calorimeter channels due to the Cherenkov pulse height. However. certain hodoscope channels had a timing shift of several nanoseconds difference between normal Cherenkov pulses and very large Cherenkov pulses (near FADC saturation).

The lower two hodoscope fingers, fingers seven and eight. in all four planes showed a timing peak, associated with these large Cherenkov events. which was about 6 ns earlier than the timing peak associated with normal height Cherenkov hits. This peak seems to be due to particles which pass through the fingers. then hit the Cherenkov PMT or the Cherenkov PMT shielding hut. Since the "good" Cherenkov hit rate at these peak heights was very low. an upper bound on the Cherenkov peak height was used in analysis to reject these events which were out of time with the other events.

\subsection{Software Corrections in the $10.5^{\circ}$ Spectrometer Analysis}

Since the $10.5^{\circ}$ spectrometer was newly constructed for E1.5.5. it was not as well understood as the other two spectrometers. As the experimental run progressed. and the understanding of the spectrometer increased, some hardware modifications were made. However, some features of the $10.5^{\circ}$ data could not be fixed through hardware modification, and required corrections in the software. 


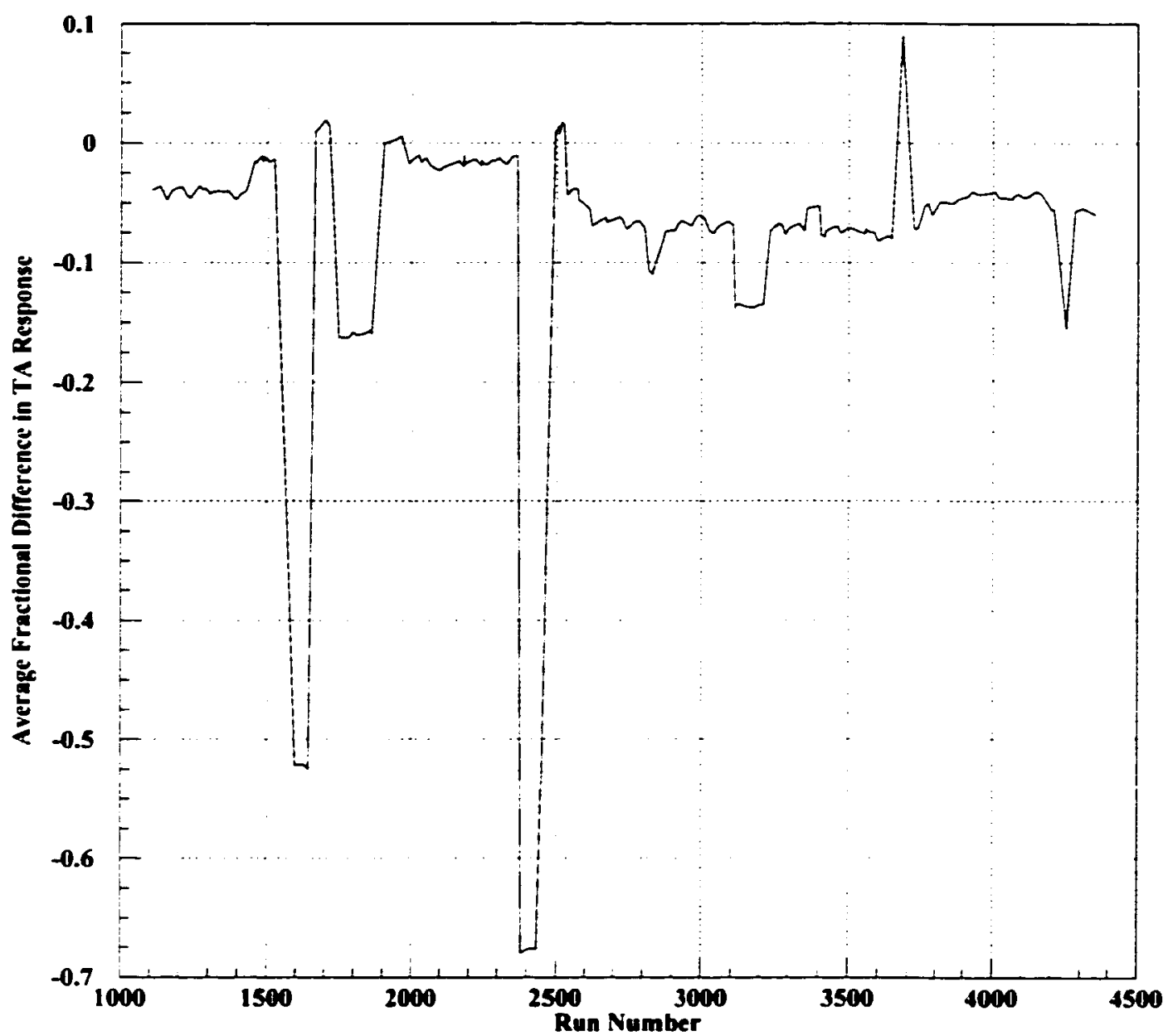

Figure 5.6: Average of the fractional difference between each TA LED response and the LED response for that TA block during Run 1902.

\subsubsection{Photomultiplier Tube Gain Variations due to Magnetic Fields}

The $\mu$-metal shields employed to protect the $10.5^{\circ}$ PMTs from the stray magnet ic fields from the spectrometer magnets were not completely effective. The shields only extended from the face of the PMT back to the base. leaving the face of the tube inadequately shielded from the fields. Sufficient field strength was present at the hodoscopes and the shower calorimeter to cause the gain to shift due to the spectrometer magnet configurations. The worst case was when the $2.7 .5^{\circ}$ spectrometer magnets were in positive polarity with the $5.5^{\circ}$ and $10.5^{\circ}$ spectrometers in negative polarity. During these running conditions the calorimeter PMT gain dropped by 
up to $90 \%$, in some blocks. with an average decrease of nearly $70 \%$. Other configurations yielded reductions of around ten percent in the TA response. Figure 5.6 shows the behavior of the average PMT signal during LED test runs throughout the experiment. The vertical axis is the average. over the TA blocks. of the fractional difference between the PMT signal during that particular run and Run $190^{\circ}$. The plot indicates the run regions during which the PMT gain was significantly lowered. The efficiency of the hodoscopes dropped in a similar manner.

Part-way through the E15.5 running, at Run 1979, the $\mu$-metal shields on the hodoscope PMTs were advanced so that they extended beyond the face of the tube by about one tube diameter. After this modification, the hodoscope fingers were not as sensitive to the magnetic environment.

The PR and TA $\mu$-metal shields also stopped at the face of the PMTs. Due to the construction of the calorimeter, the shields could not be modified to better protect the tubes. Instead, the changing response of the calorimeter PMTs was monitored using LED test runs, and the energy calibration of the shower blocks was adjusted for each configuration based upon the LED response. Figure 5.6 shows, as a function of run number, an average of the fractional difference between the LED response and a comparison LED response, taken from run 1902. The first large dip. in the run range $1573-1650$, has the $5.5^{\circ}$ in $\epsilon^{+}$mode. The next dip. over the run range $1745-1878$, has the $2.75^{\circ}$ and $5.5^{\circ}$ in $e^{+}$mode. The third large dip. over the run range $2379-2486$, has first the $2.75^{\circ}$ and $10.5^{\circ}$ in $\epsilon^{+}$mode. then the $5.5^{\circ}$ in $e^{+}$ mode. After those run ranges, all subsequent positron runs were taken with either all spectrometers in positron mode $(3113-3227.4251-4274)$, the $2.75^{\circ}$ and $5.5^{\circ}$ spectrometers both in positron mode (2812 - 28.53). or only the $10.5^{\circ}$ spectrometer in positron mode $(2515-2535,3681-3693)$. 


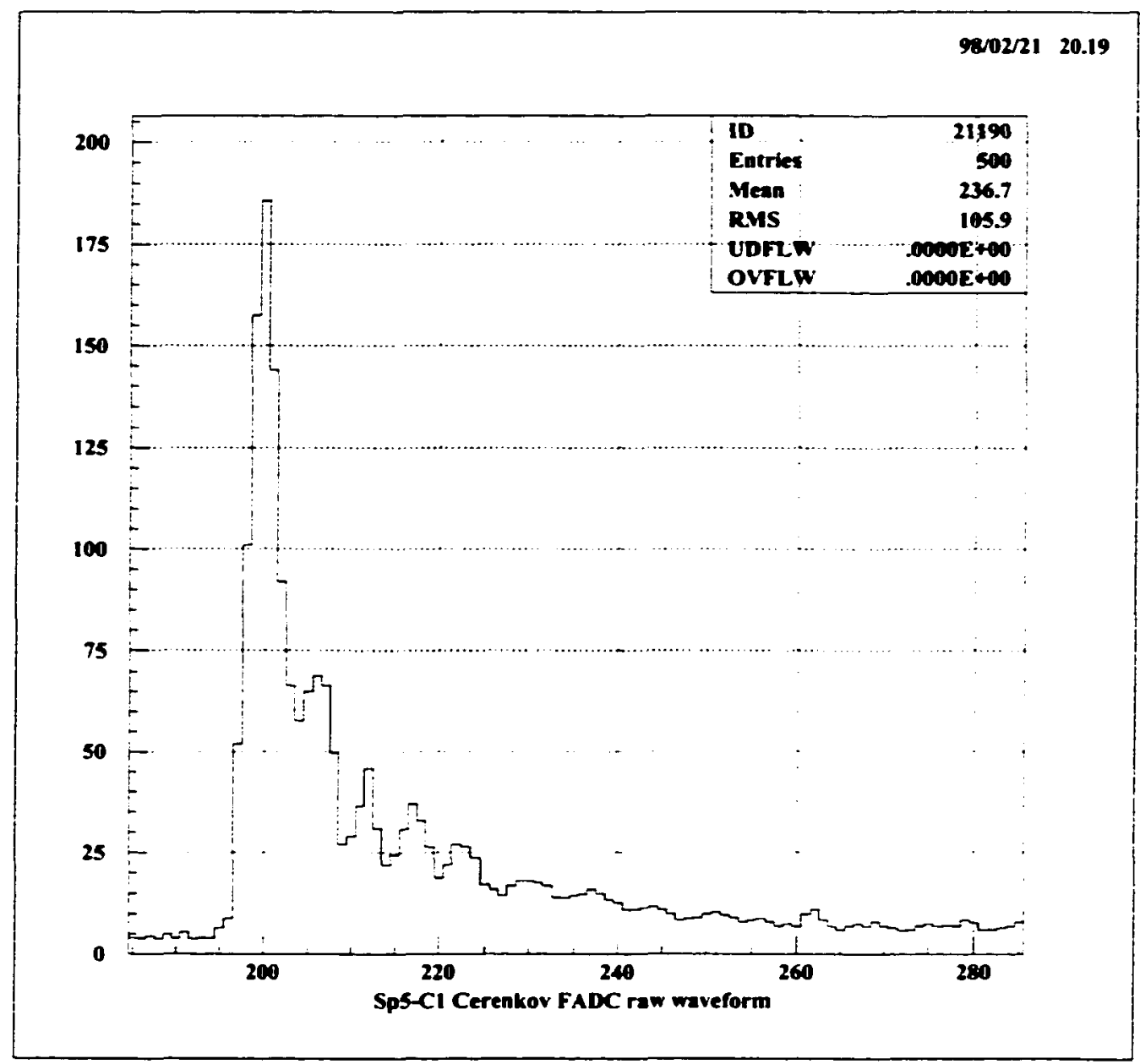

Figure 5.7: Sample FADC waveform of TA block 3.4 showing ringing on the trailing edge. This waveform was recorded during part of the T418 test run during which the ADC input for TA block 3,4 was used as the input for the FADC normally used for Cherenkov tank $5 \mathrm{Cl}$.

\subsubsection{Calorimeter Pulse Width Behavior}

The LeCroy $623 \mathrm{~B}$ discriminators used for the $10.5^{\circ}$ calorimeter were updating discriminators instead of burst-guard (time-over-threshold) discriminators. Therefore, all of the timing pulses were the same length. except for the rare cases when two small pulses would happen close enough to combine together.

By itself, this would have been just an inconvenience, but the shower calorimeter signals also suffered from ringing, as shown in Fig. 5.7. As an historical aside. I understand that clip lines were used with these PMTs when they were used in the 


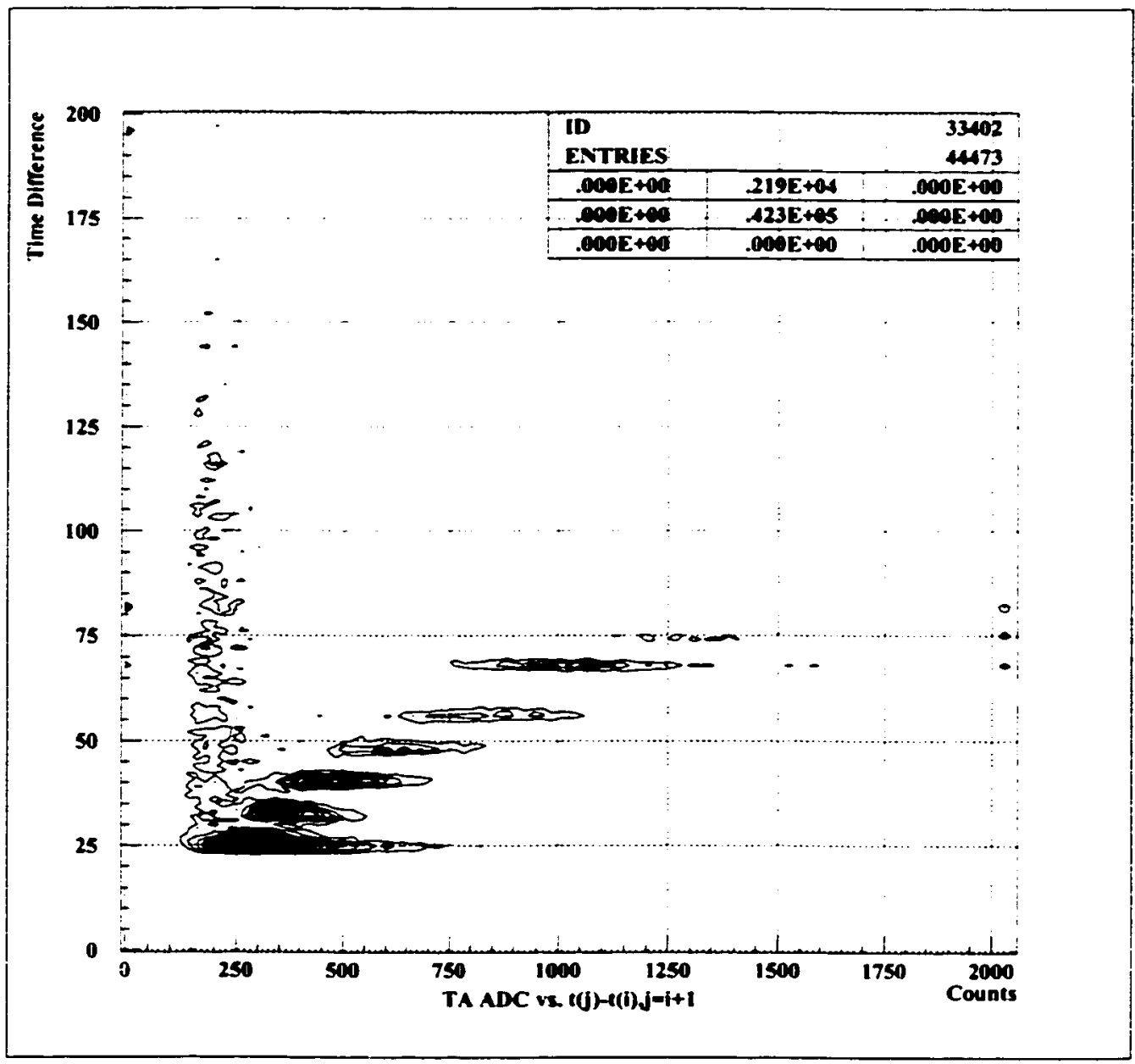

Figure 5.8: Contour plot of charge versus time difference between adjacent TDC hits. showing trends in time difference for large pulse height events. Notice that the time differences are clustered at $8 \mathrm{~ns}$ intervals (as are the ringing peaks in Fig. 5.7), and generally increases with ADC counts.

$8 \mathrm{GeV}$ spectrometer. The ringing was sufficient that the discriminator could trigger on a ring on the trailing edge of a large pulse. Therefore. large P.MT pulses were detected by the TDC modules as being two separate pulses. The shower algorithm used in the analysis of the TA (the same code as is used for the $2.75^{\circ}$ and $5.5^{\circ}$ calorimeters) uses the TDC pulse widths to separate the energy deposited in a block in each spill into individual events. Since the pulses were generally the same width. this energy sharing algorithm divided the energy into equal pieces for each TDC event. 
One proposed technique, which was never actually implemented. for interpreting these data was to use the time difference between ringing pulses to estimate the energy associated with that event. Figure 5.8 shows a structure of bands in the charge-time difference plane. By locating pairs of events with a time difference within this structure, the first event of the pair would likely be associated with the electron. In operation, the algorithm for finding these event pairs was difficult. Since the actual rate of particles in the spectrometer was small. and most multiple pulse events would consist of just an electron. it was decided that this technique was unnecessarily complex.

In the analysis, two alternate techniques were used to correct the pulse splitting effect. The first is "ghost pulse" suppression. Any event which occurs within a given time window after another event is marked as a "ghost". and is not used by the energy sharing algorithm. To completely disable the energy sharing. a ghost pulse window of $5-900$ ns was used. which caused the algorithm to ignore all subsequent pulses in the $400 \mathrm{~ns}$ spill. The second technique was to find a shower event coincident with a Cherenkov event, and call that the "real" shower event in that block for that spill. The other events centered in that block had their energy added into the selected event. The difference between the two techniques is that the second technique is able to find electrons which follow a pion or noise event in their block. The effect of using either technique in the physics analysis will be discussed in Section 5.5.1.

\subsubsection{Preradiator Discriminator Thresholds}

The preradiator response also had some problems. The high voltage settings of the preradiator were chosen to put the cosmic ray peak at about 6.5 channels above the pedestal value using a $\mathrm{qVt}$ test module, for the case when the trigger paddles were at the bar center. Cosmic signals seemed to have a peak voltage at about 50 
$\mathrm{mV}$. The pion response should have also been at about this response level.

One serious difficulty in event identification in the preradiator was the beamcurrent dependency of the ADC response: since the ADC gate was open for the duration of the spill, a higher particle rate results in a larger ADC signal. There seemed to be a large amount of noise or soft photons in the preradiator which broadened the PR energy spectra at the beam intensities used during E1.5.5. Figure 5.9 shows the energy spectra for three runs at beam currents of $1 \times 10^{9} .2 \times 10^{9}$. and $3 \times 10^{9}$ electrons per spill. Preradiator events both associated and not associated with electrons have their energies within the large peak in the spectra. There was not a clean separation between electrons and other events in the preradiator.

Due to this lack of distinction in the preradiator between electron and nonelectron events, the preradiator high voltage scans done during E1.5.5 did not provide a good set of high voltages for the tubes. In retrospect. the high voltages should probably have been higher for these tubes.

Without electron separation, the energy determination in the preradiator was confused. Initially, the large peak as seen in Fig. 5.9 was believed to be the electron response peak, and the energy calibration constants were set accordingly. When the beam current dependence of the preradiator signal was noticed. the peak seemed to indicate a large background of soft particles: thus as the beam current increased the total signal provided by the soft particles would also increase. and separation of electrons from within that mass of soft particles was not possible.

An additional problem in the analysis related to the low high voltage settings was that the discriminator threshold occurred within the peak of the ADC spectrum. Due to the low gain of the tubes, a given event was not guaranteed to produce a discriminator pulse at both ends. Figure 5.10 shows the energy spectra for three cases: the case where neither PMT on PR bar \#1 register a TDC event. the case where either PMT on PR bar \#1 registers at least one TDC event, and the case 

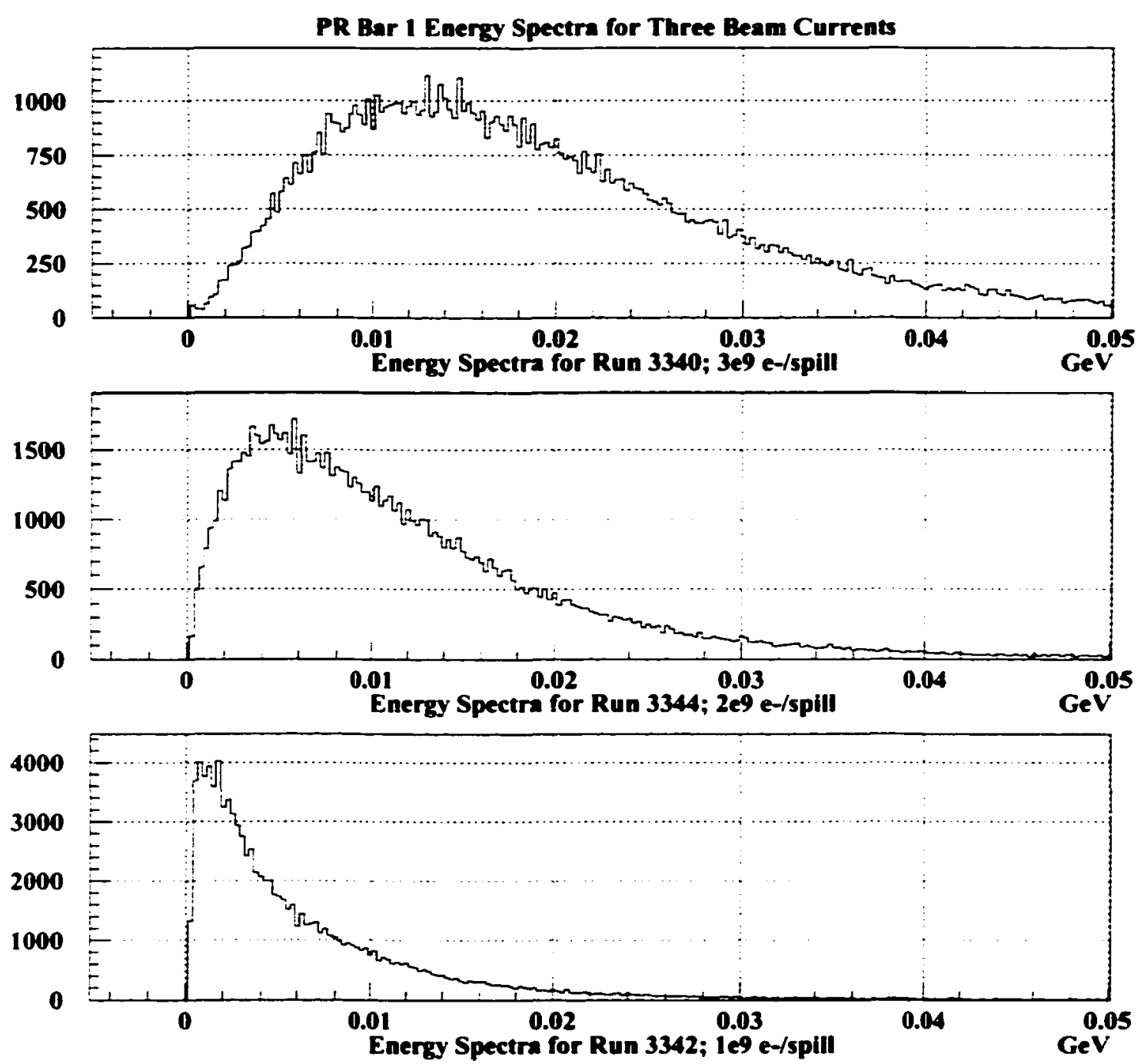

Figure 5.9: Beam current dependence of the preradiator energy spectra for currents: $1 \times$ $10^{9}, 2 \times 10^{9}$. and $3 \times 10^{9}$ electrons per spill. 


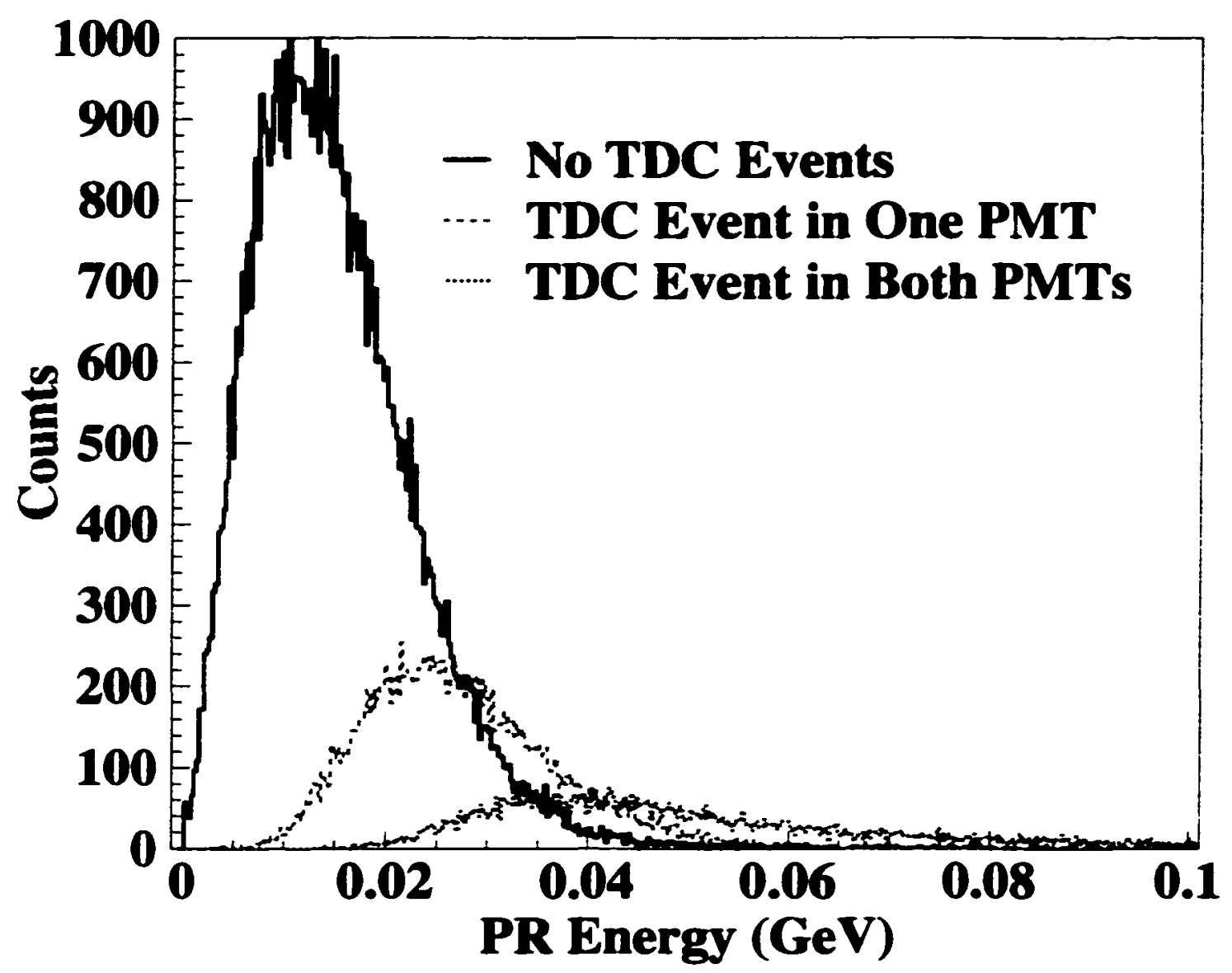

Figure 5.10: Energy spectra for Preradiator Bar 1 for: no TDC events in either PMT; at least one TDC event in one of the PMTs; at least one TDC event in both PMTs, not necessarily in coincidence. The energy scale in this plot is only approximate; this analysis was done using a preliminary preradiator calibration. The discriminator thresholds were set such that most PR ADC signals were not associated with TDC events, and most TDC events in a single PMT were not accompanied by a TDC in the opposite PMT.

where both of the PMTs register at least one TDC event. The last case does not require that the TDC events be in coincidence, however.

The energy calibration in the preradiator continued to be confusing; both due to the beam current dependence, and the low gain of the phototubes leading to an inability to cleanly separate electrons. The final analysis did not have a PR energy determination any better than what is presented in Fig. 5.9 and Fig. 5.10; however, because the energy deposition in the PR is small (energy deposited in the first two radiation lengths of a shower calorimeter is a few percent of the total energy), the 
calorimeter energy could be determined either using the PR energy or by adding a small correction for calorimeter events which did not include PR information.

\subsection{Data Summary Tape Production}

As was discussed in the beginning of the chapter. the analysis of the individual subsystems is computationally intensive. The data summary tapes (DST) contain information about the analysed events from each subsystem, so that DST ana!ysis proceeds significantly faster than analysis of raw data.

For each track, the following quantities were recorded: track class. total number of hits, number of Cherenkov hits, number of hodoscope clusters and number of fingers per cluster, $t_{0}$ (reconstructed scattering time). $P$ (scattered momentum). $\theta$ (horizontal scattering angle). $\phi$ (vertical scattering angle). $\boldsymbol{X}_{t g t}$ (position at the target). $\lambda_{X}^{2}$ (spatial chi-squared of the track). $\lambda_{t}^{2}$ (temporal chi-squared of the track). $\gamma^{2}$ (combined spatial and temporal chi-squared of the track) $, X, Y$. and the direction cosines in $X$. $Y$. and $Z$. For each Cherenkov event. the time. peak height. and integrated charge were recorded. The number of hits in each hodoscope package were recorded. For each shower cluster, the following quantities were recorded: number of blocks in the cluster, cluster class, pileup flag. edge-block flag. column and row of the central block (the central block is the block with the most energy. deposited), $E_{\text {rec }}$ (reconstructed energy from all blocks contributing to the cluster). cluster time, $X$ and $Y$, errors for $X$ and $Y$. neural network value, and energy in each of the central nine blocks.

The first pass of DST production was begun shortly after the experimental run concluded, before some features of the analysis were understood. For most of the $2.75^{\circ}$ and $5.5^{\circ}$ analyses the DST1 results could be used with corrections applied during the re-analysis. Only a limited number of $2.75^{\circ}$ and $5.5^{\circ}$ DST tapes needed to be remade. There were 101 runs with DST1b files (the DSTIb analysis corrected 
for the $4 \mathrm{~ns}$ shift in the Cherenkov counter timing), and 90 DSTlc runs (in these runs the FADC on 5C2 had failed, so the DST1c analysis used the backup system). However, the $10.5^{\circ}$ DST1 results were so affected by the issues mentioned in Section 5.3 that the results were not usable for physics analysis.

In early 1998, we decided that we had fixed enough of the problems that a second DST processing was warranted for the $10.5^{\circ}$ data. These DST2 files contained not only the event data from the Cherenkov. calorimeter. and tracking systems. but also contained the raw ADC data and TDC data from the shower calorimeter. allowing two possible $10.5^{\circ}$ re-analysis techniques. as will be discussed in Section 5.5.1. For each DST2 $10.5^{\circ}$ calorimeter event, the following quantities were recorded: number of TA blocks, number of PR blocks. central block number. PR energy. $X$ and $r$ at the PR. PR cluster time. TA energy, $X$ and $Y$ at the TA. and TA cluster time.

\subsection{DST Re-Analysis: Creation of the Summary Files}

The first step in production of the physics asymmetry is to determine the event rate in each $x$ bin for each beam helicity state. Analysis of each DST run is performed, using various event definitions. A summary file is produced for each run. giving the number of events per incident charge for the two electron beam helicities for each event definition.

Each event definition is a set of requirements, which is used to identify events of a particular type from the data stream. For each definition. an asymmetry can be generated. The principle event definitions were the standard electron and the standard pion definitions. In both of these standard definitions. a track fitting the descriptions shown in Table $\mathbf{5 . 4}$ was required. Notice that the cut on the Cherenkov voltages is done on $\sqrt{V_{C 1} V_{C 2}}$ instead of on the individual Cherenkov peak voltages: this multiplicative cut allows electron events with a small Cherenkov signal in one tank, and gives good pion separation as pion signals are generally below 40 in both 


\begin{tabular}{|l|c|c|}
\hline & Standard $e^{-}$ & Standard $\pi$ \\
\hline$x$ Range & $0.01-0.9$ & $0.01-0.9$ \\
$Q^{2}$ Range & $1.0-2.5 .0$ & - \\
Minimum $W^{2}$ & 4.0 & - \\
Track Class & 1 & 2 \\
Number of Cherenkov events & 2 & 0 \\
$E / P$ Range & $0.8-1.2$ & $0.0-0.2$ \\
$\sqrt{V_{C 1} V_{C 2} \text { Range }}$ & $40.0-500.0$ & - \\
Good Spill Range & \multicolumn{2}{|c|}{$0.0-2000.0$} \\
Bad Spill Range & \multicolumn{2}{|c|}{$0.0-2000.0$} \\
Toroid 1 Minimum & \multicolumn{2}{|c|}{0.5} \\
Toroid 2 Minimum & \multicolumn{2}{|c|}{0.5} \\
Foil Array $X$ Range & \multicolumn{2}{|c|}{$0.2-5.0$} \\
Foil Array $\sigma_{X}$ Range & $-20.0-15.0$ \\
Foil Array $Y$ Range & $0.1-5.0$ \\
Foil Array $\sigma_{Y}$ Range & \multicolumn{2}{|c|}{0} \\
\hline
\end{tabular}

Table 5.4: Standard electron and pion definitions for the $2.75^{\circ}$ and $5.5^{\circ}$ analyses. Both definitions used the tracking information to determine the event kinematics.

Cherenkov tanks.

Event definitions were constructed to count either electron-like events or pionlike events. Additional definitions sampled subsections of the electron or pion definitions to test for false asymmetries.

\subsubsection{Event Selection Analysis from DST-2}

The analysis of the DST $-210.5^{\circ}$ spectrometer data used event definitions with different criteria than for the $2.75^{\circ}$ and $5.5^{\circ}$ analyses. The first obvious difference is that the $10.5^{\circ}$ analysis needed a specification of which shower reconstruction algorithm to use. Also, since there was only one Cherenkov tank, the PR was treated as a second tank for the purposes of the multiplicative cut. so a cut was placed on the quantity $\sqrt{V_{C} E_{P R}}$. The last significant difference is the tracking system was not as efficient or as refined as it was in the other two spectrometers, therefore the tracking kinematics were not clearly the best choice.

Figure 5.11 shows spectra of four variables used in the event definition for the 

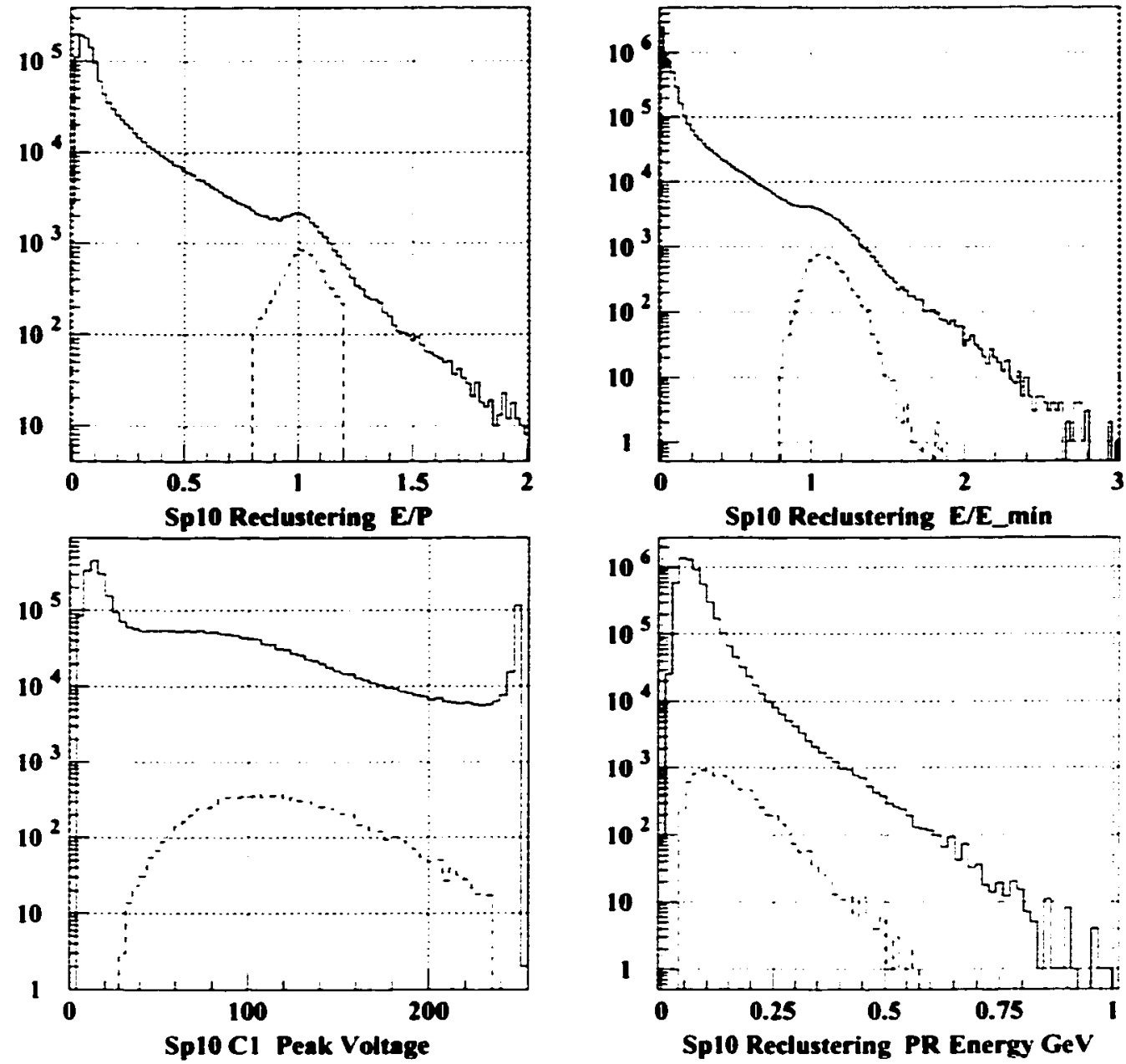

Figure 5.11: Four spectra used in event definition in the $10.5^{\circ}$ spectrometer showing the uncut (solid line) spectra and the spectra after all event definitions cuts have been applied (dashed line). These spectra contain all tracks found in seven runs in the range 2699-2i15. using a track-based definition with the reclustering shower calorimeter analysis.

$10.5^{\circ}$ spectrometer. Just as in the $2.75^{\circ}$ and $5.5^{\circ}$ analyses, a cut on the $E / P$ of $0.8-1.2$ is used. Another cut on the shower counter energy is the $E / E_{\min }$ cut; since the spectrometer has a single bend, the position of the track at the shower calorimeter serves to limit the possible momentum of the track. By placing a cut on $E / E_{\min }$ (a cut of $0.8<E / E_{\min }$ was used in Fig. 5.11), the electron signal is further developed. The peak voltage of the Cherenkov counter is used as a cut in two ways. The $10.5^{\circ}$ Cherenkov peak is generally required to have a value between 30 and $2: 35$ to eliminate the single-photoelectron signal (values less than 30 ) and the saturating 
events (values greater than 235); some definitions had a more stringent (higher value) lower limit. Also, the product of the Cherenkov peak voltage and the preradiator energy $\left(\sqrt{V_{C} E_{P R}}\right)$ is restricted: Fig. 5.11 has the cut. $4<\sqrt{l_{C} E_{P R}}<500$. where $V_{C}$ is measured in FADC units $(0-255)$, and the preradiator energy is measured in $\mathrm{GeV}$. Some definitions had a separate cut on the preradiator energy: The electron definition used in Fig. 5.11 has the requirement $E_{P R} \geq 0.04 \mathrm{GeV}$.

One of the reasons the DST2 files included the raw shower calorimeter data was to allow comparison between two methods of dealing with the multiple pulsing in the calorimeter TDCs. Rather than use the ghost pulse suppression during the DST-2 processing to only accept the first TDC events in the clustering and tracking. all TDC events were kept so that all calorimeter events could be used during the tracking process. We wrote the raw $A D C$ value for each channel and the TDC value for the leading edge of every TA or PR event to the DST2.

Since the ghost pulse suppression was disabled during DST2 production. the calorimeter events written to the DST2 file were split between each TDC event in the blocks. Thus the calorimeter energy recorded in the DST2 events was not the actual energy deposited in the physical event. Two techniques were used to reconstruct the calorimeter event energies: reclustering and recombination. The reclustering technique used the raw calorimeter data included in the DST2 records. The reclustering could use different settings for the ghost pulse suppression. The standard reclustering analysis used a ghost pulse window of $5-900$ ns: i.e., any second pulse occurring between $5 \mathrm{~ns}$ to $900 \mathrm{~ns}$ after the initial pulse was discarded. No second pulse could occur before about $25 \mathrm{~ns}$, so the lower cut of $5 \mathrm{~ns}$ was conservative. Notice that the upper bound of $900 \mathrm{~ns}$ was beyond the electronics gate and the spill length, so all subsequent pulses in a block were discarded. This analysis therefore selected the first TDC event in each block as the "real" event. Once the reclustering analysis was complete, the new clusters were matched to existing tracks 


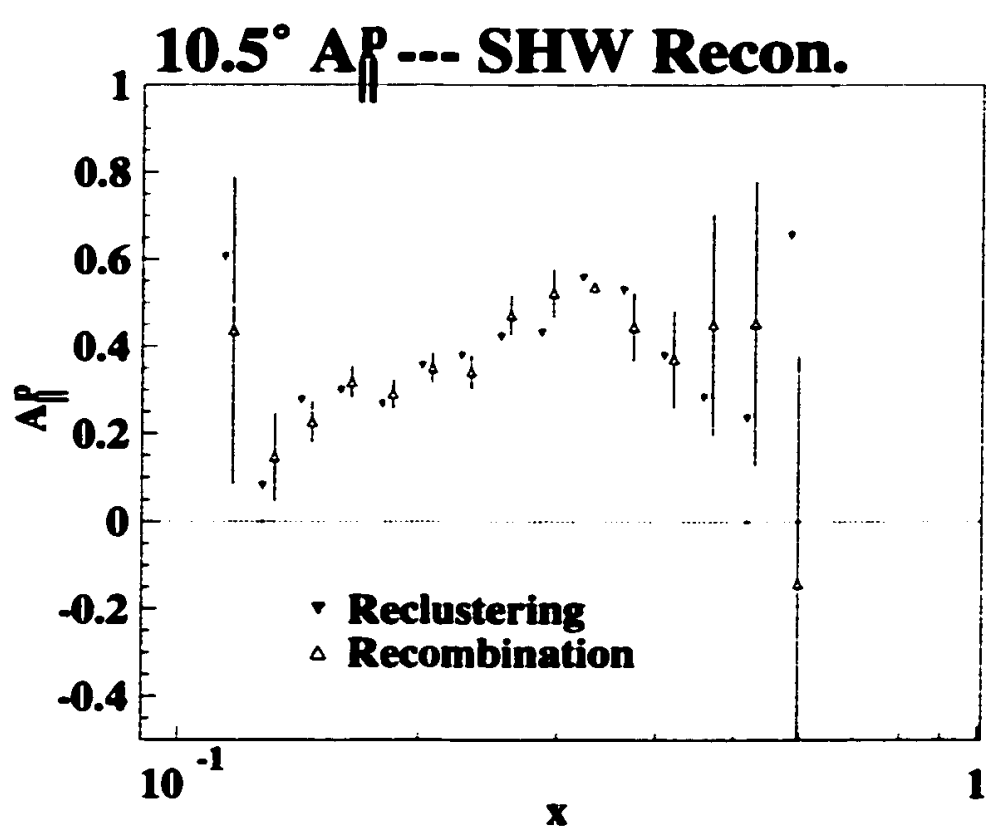

Figure 5.12: Comparison of $A_{\|}^{p}$ using either the recombination or reclustering shower calorimeter analyses. The two definitions used are otherwise identical, except for the choice of recombination or reclustering. Both definitions are track-based, and $\boldsymbol{x}$ is determined from track momentum. The points have been shifted in $x$ for clarity; the true $x$ of each bin lies between the data points. All corrections have been applied, except for radiative corrections. The asymmetries agree within their errors. Systematic errors are not shown.

and Cherenkov events. In recombination, events centered on the same TA block were checked for time coincidence with Cherenkov or PR events to determine which event was the "true" particle, then the energy in the other event was added to the energy of the true event. This addition would take place even if the "true" event occurred later than the other event; the rationale was that the energy of the other event would be dominated by the fraction of the true event's energy. This analysis did not use the raw calorimeter data, and was somewhat faster than the reclustering technique.

During re-analysis, either recombination or reclustering could be used, allowing comparison of the physics result. Both techniques produce consistent physics results, as shown in Fig. 5.12.

Similarly, re-analysis could be based upon tracks or showers; the track-based analyses require a Class 1 track (containing a shower and Cherenkov event), and the 


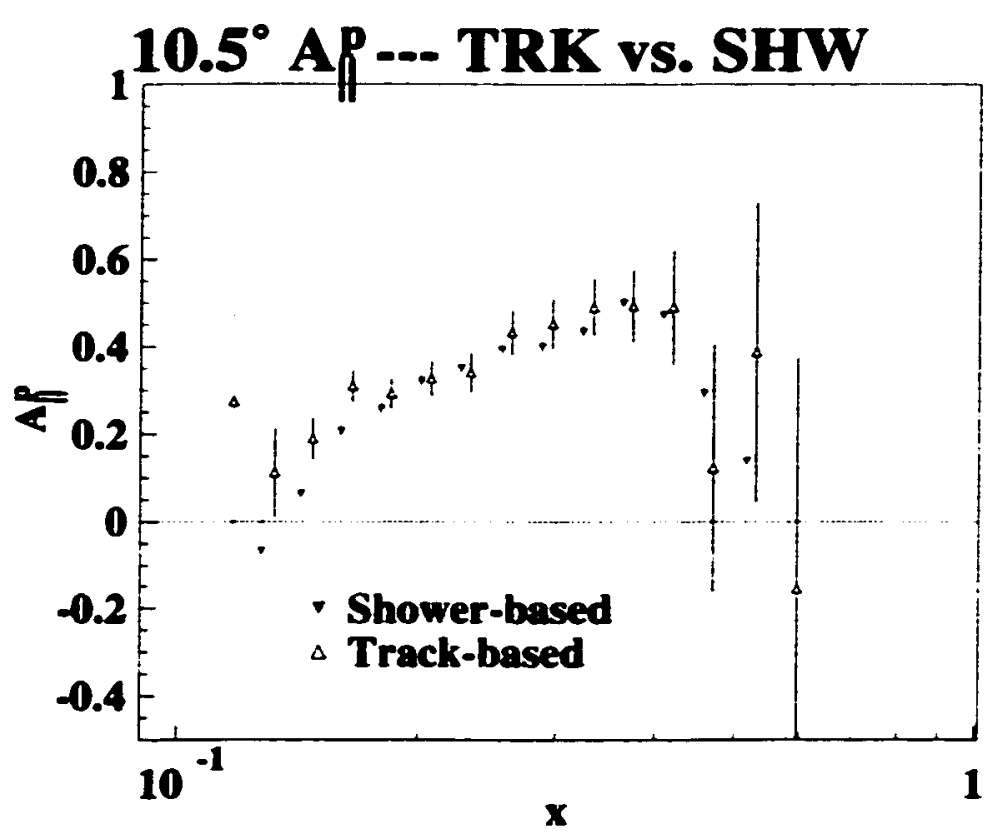

Figure 5.13: Comparison of $A_{\| 1}^{p}$ using either the track-based or shower-based analyses. In both definitions, the shower calorimeter energy is used to determine $x$ and $Q^{2}$ : the recombination shower analysis is used. The two definitions used are otherwise identical. The lowest $x$ bin of the shower-based definition had no valid events after the contamination correction; therefore, it has not been included on the plot. The points have been shifted in $x$ for clarity; the true $\boldsymbol{x}$ of each bin lies between the data points. All corrections have been applied, except for radiative corrections. The asymmetries agree within their errors. Systematic errors are not shown.

shower-based analyses require a high energy shower in time coincidence with a Cherenkov event (coincidence with hodoscope events or tracks is not required). Using shower-based definitions allowed somewhat higher statistics, but increased the pionpositron contamination as compared to the track-based definitions; this is shown in Fig. 5.19 of Section 5.6.2. Figure 5.13 shows $A_{\|}^{p}$ for the track-based and showerbased analyses; they are in reasonable agreement, but the shower based analysis is somewhat smaller in the low $x$ region, perhaps due to incomplete contamination correction.

Also, the kinematic parameters $x$ and $Q^{2}$ may be determined using either the tracking momentum and scattering angle information, or the shower calorimeter energy and shower position information. The tracking resolution is about $0.007 E^{\prime}$ 


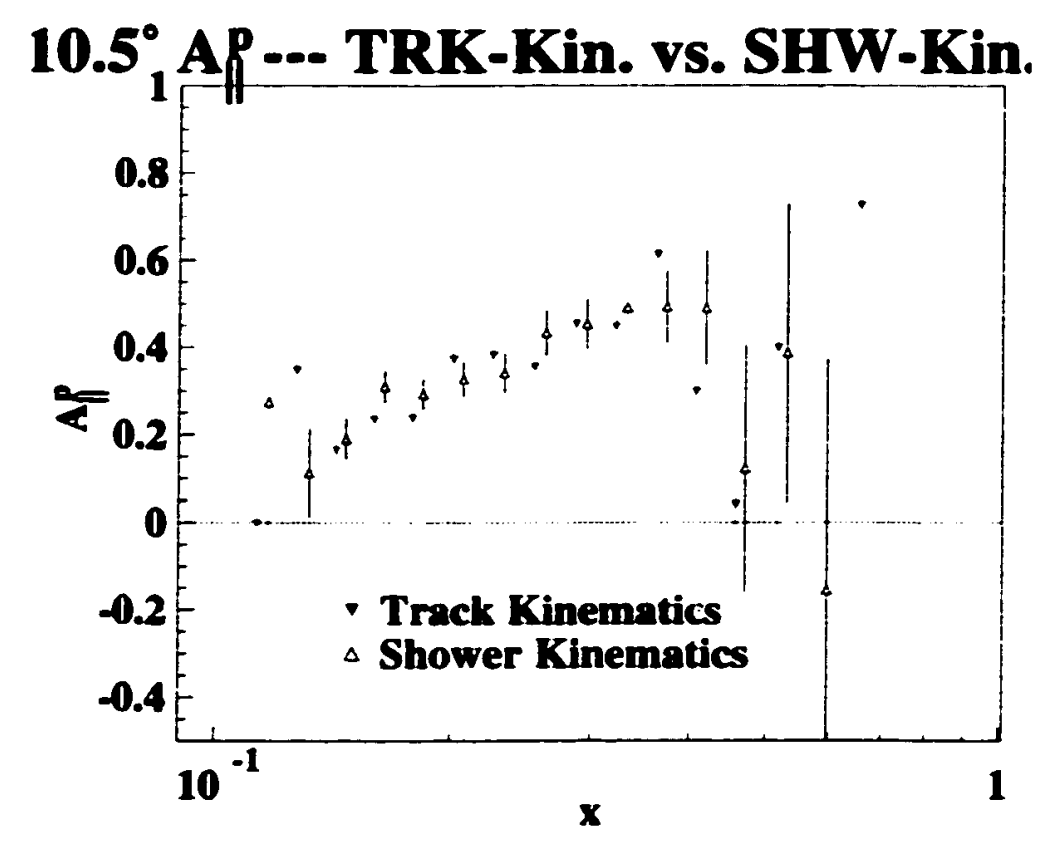

Figure 5.14: Comparison of $A_{\|}^{p}$ using either the track momentum or shower energy to determine $x$ and $Q^{2}$. Both definitions are track-based. and the recombination shower analysis is used in both. The two definitions used are otherwise identical. The points have been shifted in $x$ for clarity: the true $x$ of each bin lies between the data points. All corrections have been applied, except for radiative corrections. Systematic errors are not shown.

(where $E^{\prime}$ is measured in $\mathrm{GeV}$ ), with large granularity effects [120]. The energy resolution of the shower calorimeter blocks was measured in previous experiments to be about $0.1 / \sqrt{E^{\prime}}[120]$, although errors in the block-to-block energy calibration may worsen this somewhat in the $\mathrm{E} 155$ calorimeter. The $E / P$ window of 0.8 to 1.2 gives a potential for smearing of events across several $x$-bins, which may account for some of the differences seen in Fig. 5.14.

\section{The "Hybrid" Definition}

The final physics analysis for the $10.5^{\circ}$ spectrometer utilized the "hybrid" definition $[120,121]$. The hybrid definition uses the recombination method of reprocessing the calorimeter events, and utilizes both track-based and shower-based subdefinitions, resulting in higher statistics than either the standard track-based or showerbased definitions. Additionally, the hybrid definition uses the calorimeter energy 


\begin{tabular}{|c|}
\hline Subdefinition I \\
\hline $\begin{array}{l}\text { Class } 1 \text { track } \\
\left(E_{S H W}>7 \text { and no ADC saturation) or }\left(P>7 \text { and } E_{S H W}>5\right)\right. \\
E_{S H W} / P>0.75 \\
\left.V_{C}>45 \text { ( } 30 \text { for runs before Run } 2103\right) \\
\text { Timing coincidence between } \mathrm{TA} \text { and Cherenkov of } \pm 3 \mathrm{~ns}\end{array}$ \\
\hline $\begin{array}{ll}\text { Subdefinition II } \\
\end{array}$ \\
\hline $\begin{array}{l}\text { Failed Subdefinition I } \\
\text { Class } 1 \text { track } \\
\left(E_{S H W}>7 \text { and no } A D C \text { saturation }\right) \text { or }\left(P>\imath \text { and } E_{S H W}>5\right) \\
E_{S H W} / E_{\min }>0.9 \\
V_{C}>60(40 \text { for runs before Run } 2103) \\
\text { Timing coincidence between TA and Cherenkov of } \pm 3 \mathrm{~ns} \\
\end{array}$ \\
\hline $\begin{array}{ll} & \text { Subdefinition III } \\
\end{array}$ \\
\hline $\begin{array}{l}\text { Failed Subdefinition II } \\
\text { No ADC saturation } \\
E_{S H W}>7 \\
E / E_{\min }>0.9 \\
\left.V_{C}>60 \text { (40 for runs before Run } 2103\right) \\
\text { Timing coincidence between TA and Cherenkov of } \pm 3 \mathrm{~ns}\end{array}$ \\
\hline
\end{tabular}

Table 5.5: The three subdefinitions of the student implementation of the $10.5^{\circ}$ hybrid definition.

(unless ADC saturation occurred in the shower calorimeter) to determine the $x$ and $Q^{2}$, instead of using the tracking momentum [120]. The implementation of the hybrid definition in the student analysis utilized the three subdefinitions shown in Table 5.5 [121]. The hybrid definition did not require the calorimeter events to have PR information.

\subsection{Producing the DIS Asymmetries. $A_{\|} \& A_{\perp}$}

Once the summary files are produced, they can be processed with the physics analysis to determine the raw asymmetry measured for a particular definition. Corrections are applied to the raw asymmetry to yield the deep inelastic asymmetries $A_{\|}$and $A_{\perp}$ from the longitudinal and transverse data.

In the physics analysis, the raw asymmetry for each data run is determined 
and the asymmetries for similar runs are averaged together to produce the raw asymmetries $A_{\|, r a w}^{p}, A_{\perp, r a w}^{p}, A_{\|, \text {raw }}^{d}$ and $A_{\perp . r a w}^{d}$. Additional corrections are applied to these asymmetries to produce the final $A_{\|}$and $A_{\perp}$ asymmetries for the proton and deuteron.

\subsubsection{Raw Asymmetry Production: Asymmetries for Each Run}

The summary files contain the number of events in each definition, binned by $x$ and beam helicity. They also contain the total charge incident on the target for a particular definition and beam helicity. Also contained are the summed values of $x$ and $Q^{2}$ for all the events in each definition and $x$ bin. From this information. a raw asymmetry for the definition is constructed as a function of $x$ bin.

$$
A_{\text {raw }}^{\text {run }}=\frac{S}{P_{\text {beam }} P_{\text {target }} f C_{1}}\left(\frac{R_{\text {left }}-R_{\text {right }}}{R_{\text {left }}+R_{\text {right }}}+A_{R D}-P_{\text {beam }} \cdot A_{e w}\right)+C_{2} A_{\text {other }} .
$$

where $R_{\text {left(right) }}=N_{\text {left(right) }} / Q_{\text {left(right) }}$ is the number of events normalized by incident beam charge for left-handed (right-handed) beam helicity: $P_{\text {beam }}$ and $P_{\text {target }}$ are the beam and target polarizations, $f$ is the dilution factor. $C_{1}$ and $C_{2} f_{\text {other }}$ are nuclear correction terms. $A_{e w}$ is the electroweak asymmetry. and $f_{R D}$ is the asymmetry due to rate dependence effects. The factor. $S^{\prime}= \pm 1$. is a sign factor to adjust for the target field direction. The statistical error on the asymmetry for each run is approximately,

$$
\sigma_{\text {Arau }}=\left|\frac{1}{P_{\text {beam }} P_{\text {target }} f C_{1}} \frac{\sqrt{R_{\text {left }} / Q_{\text {left }}+R_{\text {right }} / Q_{\text {right }}}}{R_{\text {left }}+R_{\text {right }}}\right| .
$$

\section{Target Polarization \& Field Orientation}

The target polarization was measured regularly throughout each data collection run, and reported to the main data acquisition system from the target control computer. The target polarization did not vary significantly during a given data collection run, so the average target polarization during each run was used to calculate 
the asymmetry for the run. The target polarization information was re-analyzed after the E155 experimental run, and the results from that analysis were provided to the E15.5 physics analysis in the form of a lookup table for the average target polarization during each data collection run.

For the proton target, the target polarization measurement was troubled by stray capacitance. Three indications of problems with the proton target NMR measurements were observed: the signals were inverted. measured polarizations were as high as $111 \%$, and the signal was very small and distorted when an aluminium (actually copper-coated aluminium) NMR coil was used [122]. The inversion of the signal and observation of polarizations greater than $100 \%$ were due to stray capacitance between the N.MR coil, the $\mathrm{NH}_{3}$, and the metal target cup: only the proton NMR measurements seem to have been affected. due to its large magnetic moment (the proton NMR frequency at $5 \mathrm{~T}$ is $213 \mathrm{MHz}$ ) as compared to the magnetic moment of the other nuclei (the ${ }^{15} \mathrm{~N}$ NMR frequency is $20.7 \mathrm{MHz}$ in the same field) [123]. Analysis of the average parallel asymmetry for each target cell revealed that the cells with an aluminium NMR coil had asymmetries different from the other data by several sigma [122], from which it was decided not to use the asymmetry data from the cups with an aluminium NMR coil, constituting about $10-1.5 \%$ of the proton data. for the physics analysis [122]. A similar analysis of average parallel asymmetry versus target polarization indicated a decrease in the asymmetry in the data with high observed polarization [122]. A variety of interim corrections to the data were attempted [123. 124], such as using nitrogen polarizations to normalize the proton data, using an assumed form of the polarization decay due to radiation damage, or trying to model the observed form of the NMR signal.

The final proton polarization correction [112] uses the observed polarization decay due to radiation damage, and uses the accumulated beam dose to relate the flawed E15.5 polarization to the polarization measured in E1.5.5X; the E15.5X tar- 
get inserts used non-metallic target cells and were not affected by the problems of the E15.5 target inserts. This correction is also applicable to the target cells with aluminium coils, allowing the inclusion of those data in the final proton analysis [124]. Using this correction, the parallel asymmetries for each target cell are consistent with each other, with only a slight slope in the average asymmetry versus polarization [124].

During the parallel asymmetry measurement, the target magnetic field was either aligned pointing east, parallel to the beam direction, or pointing west, antiparallel to the beam direction. For a particular magnetic field direction. the target was polarized with either positive or negative enhancement, where positive enhancement indicates nuclear alignment along the field and negative enhancement is anti-aligned along the field. The four possible combinations of target field ( $E$ or $W$ ) and polarization enhancement $(+$ or - ) yield two ways to align the nucleons parallel $(\mathrm{E}+$ and $W-$ ) or antiparallel $(E-$ and $W+$ ) to the beam direction. Changing the field direction introduces a sign change in the extraction of the physics asymmetry using Eq. 5.11: the sign factor is $S=+1$ for the field aligned east. and $S=-1$ for the field aligned west. Recasting Eq. 5.11 to give the counting rate asymmetry shows.

$$
A_{\text {meas }}=\frac{P_{\text {beam }} P_{\text {target }} f C_{1}}{S}\left(A_{D I S}-C_{2} \cdot A_{\text {other }}\right)+P_{\text {beam }} \cdot A_{e w}
$$

where $A_{\text {meas }}=\left(R_{\text {left }}-R_{\text {right }}\right) /\left(R_{\text {left }}+R_{\text {right }}\right)+A_{R D}$ is the counting rate asymmetry corrected for rate dependence, and $A_{D I S}$ is either $A_{\|}^{p}$ or $A_{\| l}^{d}$. Notice that by changing either the target field direction or the polarization enhancement, the contribution to the counting rate asymmetry from the DIS process changes sign but the electroweak asymmetry does not; the difference between the asymmetries for east and west target field alignment, $A_{\text {meas }}^{\text {east }}-A_{\text {meas }}^{\text {west }}$, does not depend upon the electroweak asymmetry.

In practice, the asymmetries for each target polarization direction were corrected for the electroweak asymmetry (see Section 5.6.1). but by collecting equal 
amounts of data with opposite target alignments and taking the weighted average of the extracted DIS asymmetries, beam helicity related systematic errors cancel. Most of the E15.5 data collection was done in the positive enhancement. with about equal amounts of data with the field oriented to the east and to the west. The extracted DIS asymmetries for the two field directions are in good agreement [125].

Duririg the perpendicular asymmetry measurement. the target field was always aligned north with positive enhancement. The perpendicular asymmetry had a sign term which depended upon the direction of the horizontal scattering angle. The $5.5^{\circ}$ spectrometer was on the north side of the beam line. so the angle between the scattering plane and the polarization plane. $O$ from Eq. 1.35 is zero. yielding a positive sign to the asymmetry; the $2.75^{\circ}$ and $10.5^{\circ}$ spectrometers are on the south side of the beam line, so $\phi \approx \pi$, giving a negative sign.

\section{Beam Polarization}

The beam polarization was taken as $0.810 \pm 0.020$. as described in Section 2.2.2. The Møller polarimetry measurements did not indicate any significant variation in polarization over the course of the experiment, so $P_{B}$ was taken to be constant for all of the E15.5 analysis.

\section{Dilution Factor and Nuclear Corrections}

Not all of the scattering events originate at the protons or deuterons in the polarized target: scattering may occur from nuclei, polarized or unpolarized, of other materials present in the target or beam line [127. 126]. The spectrometer counting rates have contributions not only from the unpolarized cross-section. $\sigma_{p(d)}$. and asymmetry, $A_{\|}^{p(d)}$, but also from the cross-sections and asymmetries of all other nuclei present within the spectrometer acceptance. To extract $A_{\|}^{p(d)}$ from the measured asymmetry, two types of corrections are required: a dilution factor, which corrects 


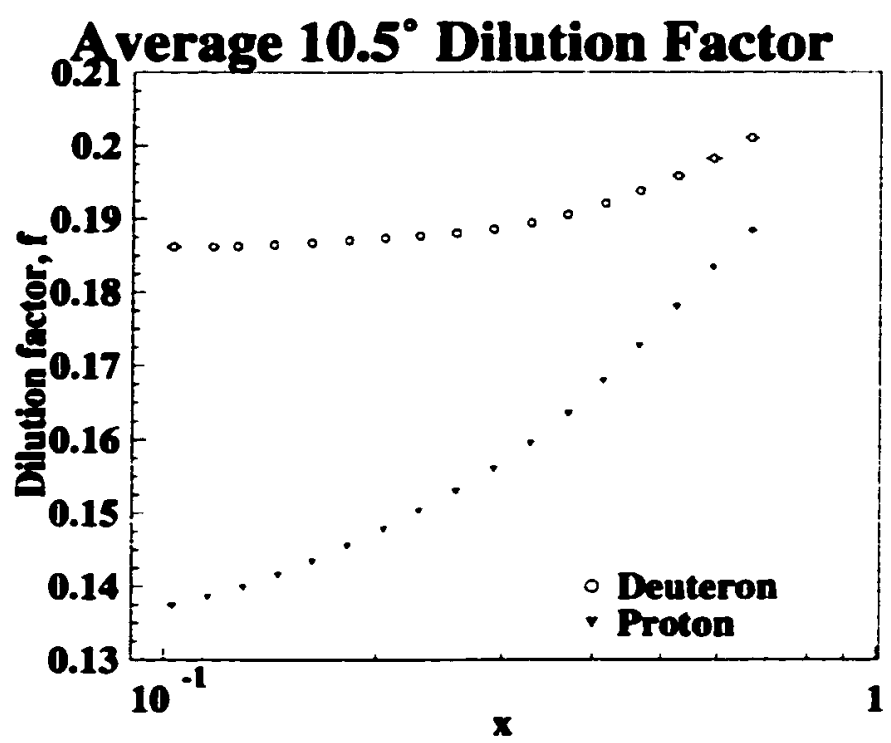

Figure 5.15: Average dilution factor in the $10.5^{\circ}$ spectrometer in each $x$-bin for the proton and deuteron target materials. The dilution factor calculated here is based upon the code introduced in Reference [126] rather than the dilution factor determined from the E155 dataset: the dilution factors calculated from $\mathbf{E 1 5 5}$ data are similar.

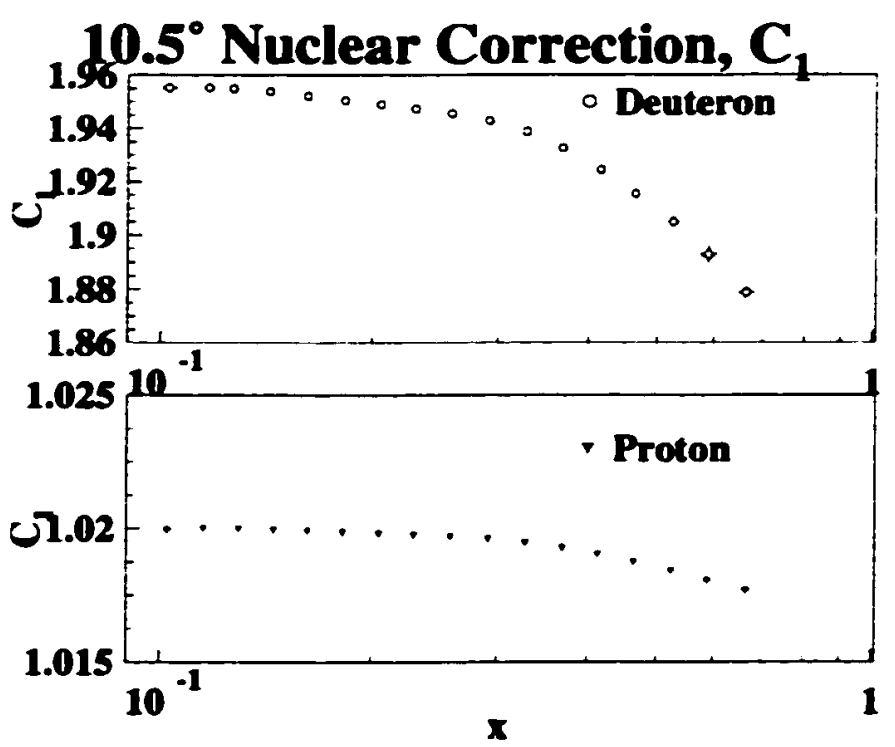

Figure 5.16: Average multiplicative nuclear correction term, $C_{1}$, in each $x$-bin for the proton and deuteron target materials. The nuclear correction calculated here is based upon the code introduced in Reference [126] rather than the nuclear correction determined from the E15j dataset; the nuclear corrections calculated from E155 data are similar. 
the unpolarized counting rate, and nuclear corrections, which correct the measured asymmetry for contributions from other polarized species [126]. Consider the general form of the measured rate in an acceptance $\Phi$ for left-handed (right-handed) helicity electrons on a nuclear target with other polarized and unpolarized nuclei present $[12 T, 126]$,

$$
\begin{aligned}
L(R)= & \Phi\left[N_{\text {nucleon }} \sigma_{\text {nucleon }}^{\text {rad }}\left(1 \pm A_{\text {nucleon }} P_{b} P_{\text {nucleon }}\right)\right. \\
& \left.+\Sigma_{j} N_{j} \sigma_{j}^{\text {rad }}\left(1 \pm A_{j} P_{b} P_{j}\right)+\Sigma_{i} N_{i} \sigma_{i}^{\text {rad }}\right]
\end{aligned}
$$

where the nucleon subscript indicates either protons or deuterons. the sum over $j$ includes all additional polarized nuclear species. the sum over $i$ includes all unpolarized nuclear species, $P_{b}$ is the beam polarization. $\nu_{X}$ is the number density of nuclei $X, \sigma_{X}^{r a d}$ is the unpolarized cross-section including radiative effects. $A_{X}$ is the DIS asymmetry. and $P_{X}$ is the polarization of nuclei $X$. The measured asymmetry is.

$$
\frac{L-R}{L+R}=P_{b} P_{\text {nucleon }} f\left[A_{\text {nucleon }}+\Sigma_{j} \frac{P_{j}}{P_{\text {nucleon }}} \frac{N_{j} \sigma_{j}^{r a d}}{N_{\text {nucleon }} \sigma_{\text {nucleon }}^{r a d}} A_{j}\right]
$$

where the dilution factor. $f$. is defined as.

$$
f=\frac{N_{\text {nucleon }} \sigma_{n \text { nucleon }}^{\text {rad }}}{N_{\text {nucleon }} \sigma_{\text {nucleon }}^{\text {rad }}+\Sigma_{j} N_{j} \sigma_{j}^{\text {rad }}+\Sigma_{i} N_{i} \sigma_{i}^{r a d}} .
$$

The radiated cross-sections are determined from the Born cross-sections and radiative corrections [127].

In the ${ }^{15} \mathrm{NH}_{3}$ target, the polarized nuclei present are protons. ${ }^{15} \mathrm{~N}$, and a small amount of ${ }^{14} \mathrm{~N}$ (deuterons make up about $0.015 \%$ of the total hydrogen [128] so have a negligible effect). The total number density of nitrogen will be expressed as $\lambda_{x}$. with $\eta_{N}$ indicating the ${ }^{14} \mathrm{~N}$ fraction. Then Eq. 5.15 is [126].

$$
\frac{L-R}{L+R}=P_{b} P_{p} f\left[A_{p}+\left(1-\eta_{N}\right) \frac{N_{N}}{N_{p}} \frac{P_{15}}{P_{p}} \frac{\sigma_{15}^{r a d}}{\sigma_{p}^{r a d}} A_{15}+\eta_{N} \frac{N_{N}}{N_{p}} \frac{P_{14}}{P_{p}} \frac{\sigma_{14}^{r a d}}{\sigma_{p}^{r a d}} A_{14}\right]
$$


where the subscripts 14 and 15 refer to ${ }^{14} \mathrm{~N}$ and ${ }^{15} \mathrm{~N}$. The dilution factor is [126].

$$
f=\frac{N_{p} \sigma_{p}^{r a d}}{N_{p} \sigma_{p}^{r a d}+\left(1-\eta_{N}\right) N_{N} \sigma_{15}^{r a d}+\eta_{N} N_{N} \sigma_{14}^{r a d}+\Sigma_{i} N_{i} \sigma_{i}^{r a d}}
$$

and is shown in Fig. 5.15 for the $10.5^{\circ}$ spectrometer. The nitrogen contributions are assumed to be [126]:

$$
\begin{aligned}
& A_{15} \sigma_{15}^{r a d}=\beta_{15} g_{15} A_{p} \sigma_{p}^{r a d} \\
& A_{14} \sigma_{14}^{r a d}=\beta_{14} g_{14}\left(A_{p} \sigma_{p}^{r a d}+A_{n} \sigma_{n}^{r a d}\right) .
\end{aligned}
$$

where the 3 correct for nuclear effects and the $g$ correct for the EMC effect in nitrogen, which is a modification of the nucleon structure functions within the nucleus [129]. $A_{14} \sigma_{14}^{\text {rad }}$ can be rewritten in terms of the deuteron by using $A_{D} \sigma_{D}^{\text {rad }}=$ $\hat{i}_{D}\left(A_{p} \sigma_{p}^{r a d}+A_{n} \sigma_{n}^{r a d}\right)$. where $\gamma_{D}$ corrects for the $D$-state of the deuteron.

$$
A_{14} \sigma_{14}^{r a d}=\beta_{14} g_{14} A_{D} \sigma_{D}^{r a d} / \gamma_{D}
$$

Then solving Eq. 5.17 for $A_{p}$.

$$
A_{p}=\frac{\frac{L-R}{L+R}}{P_{p} P_{b} f C_{1}}+C_{2} \frac{\sigma_{D}^{r a d}}{\sigma_{p}^{r a d}} \cdot A_{D}
$$

The nuclear correction terms are.

$$
\begin{aligned}
& C_{1}=1+\left(1-\eta_{N}\right) \frac{N_{N}}{N_{p}} \frac{P_{15}}{P_{p}} \beta_{15} g_{15} \\
& C_{2}=-\frac{1}{C_{1}}\left[\eta_{N} \frac{N_{N}}{N_{p}} \frac{P_{14}}{P_{p}} \frac{\beta_{14}}{\gamma_{D}} g_{14}\right] .
\end{aligned}
$$

where $\beta_{15}=-1 / 3, \frac{N_{V}}{N_{p}}=1 / 3, g_{15} \approx 1$, and a fit done by E143 of ${ }^{15} \mathrm{~N}$ polarization as a function of proton polarization was used for $P_{15}[28]$.

$$
P_{15}=-\left(0.136 P_{p}-0.183 P_{p}^{2}+0.335 P_{p}^{3}\right)
$$

The ${ }^{14} \mathrm{~N}$ fraction was about $\eta_{N}=0.02$ [128], allowing the approximation $C_{2} \equiv 0$. The nuclear correction, $C_{1}$, in the $10.5^{\circ}$ spectrometer for the proton target is shown in Fig. 5.16. 
The LiD target material was dominantly composed of ${ }^{6} \mathrm{Li}$ and ${ }^{2} \mathrm{H}$, with a residual ' $\mathrm{Li}$ fraction of $\eta_{L}=0.046$ and a residual proton fraction of $\eta_{p}=0.025$ [128]. The dilution factor and nuclear corrections for LiD have the same form as for the $\mathrm{NH}_{3}$. except there are more polarized species present. The ${ }^{6} \mathrm{Li}$ acts like a deuteron plus ${ }^{4} \mathrm{He}$, and the ${ }^{7} \mathrm{Li}$ has an asymmetry related to the proton asymmetry. Both nuclear correction terms are needed for the $\mathrm{LiD}$ target. The dilution factor and $C_{1}$ nuclear correction terms for the $10.5^{\circ}$ spectrometer are shown in Figures 5.15 and 5.16 . respectively.

The number densities of nuclei within the target cup (the liquid He bath and either the $\mathrm{NH}_{3}$ or LID) depend upon the packing fraction. which is the fraction of the target cup volume which is occupied by granules of target material. This is determined experimentally by comparing the scattering rates of the polarized target with those of the corresponding unpolarized solid target (comparing the $\mathrm{NH}_{3}$ rates to $\mathrm{C}$. or the $\mathrm{LiD}$ rates to $\mathrm{Be}$ ). The packing fractions were all found to be in the range $0.52-0.64[130]$.

The target model. a simulation of the target, is used in the development of the dilution factor, nuclear corrections, and the radiative corrections (see Section 5.6.4). The target model describes the number of radiation lengths of all materials before and after the target, so that the probability of radiation in the material may be calculated. Included are such elements as: vacuum windows on the beam-line and target assembly, liquid helium surrounding the target cell. target cell windows. the target material, the NMR coils in the target, and the helium bag which follows the target.

\section{Rate Dependence}

It is known [131] that number of reconstructed electrons per incident beam charge in the spectrometers is dependent upon the luminosity. and that the lu- 
minosity dependence is particularly strong in the $10.5^{\circ}$ spectrometer. The rate dependence correction is not based upon the luminosity, but upon the number of events per spill in each detector subsystem.

The rate dependence correction adjusts the measured counting rates for the two beam helicity states for the effects of dead time. If the detectors are counting at a high rate, the possibility that an event will occur within the detector dead time from the preceding event increases. The rate dependence of the E1.5.5 data was studied [47] using the 'pulse fiction' method of E154 [23]. Higher rate data collection is simulated by adding the raw data from successive spills together taking the detector dead time into account. The efficiency of electron reconstruction in this simulated double-rate data is compared to the electron reconstruction efficiency at the actual rate to determine the effect on the asymmetry. This merging is done for each detector subsystem (hodoscopes, Cherenkov, shower calorimeter) to determine the effect on the asymmetry due to rates in each detector system. A small rate dependence correction was made to the $2.75^{\circ}$ and $5.5^{\circ}$ data. which increased the asymmetries by about $1 \%[4 \pi]$. No rate dependence correction was made to the $10.5^{\circ}$ spectrometer data, because the event rates in the detectors were low.

\section{Electroweak Correction}

In scattering of polarized electrons, $\vec{e}+N \rightarrow \epsilon+X$. there is an asymmetry which arises from interference between photon and $Z^{0}$ exchange terms in electroweak interactions. The electroweak asymmetry deperds upon the helicity of the incident electrons. and not upon the target polarization.

The E1.55 electroweak asymmetry calculation has the form [132]

$$
A_{E W} \approx-10^{-4} Q^{2}\left(0.77\left[1+0.44 R_{s}(x)\right]+0.11 Y\right)
$$

where $Q^{2}$ is the four-momentum transfer squared in $(\mathrm{GeV} / \mathrm{c})^{2}, R_{s}(x)$ is related to 


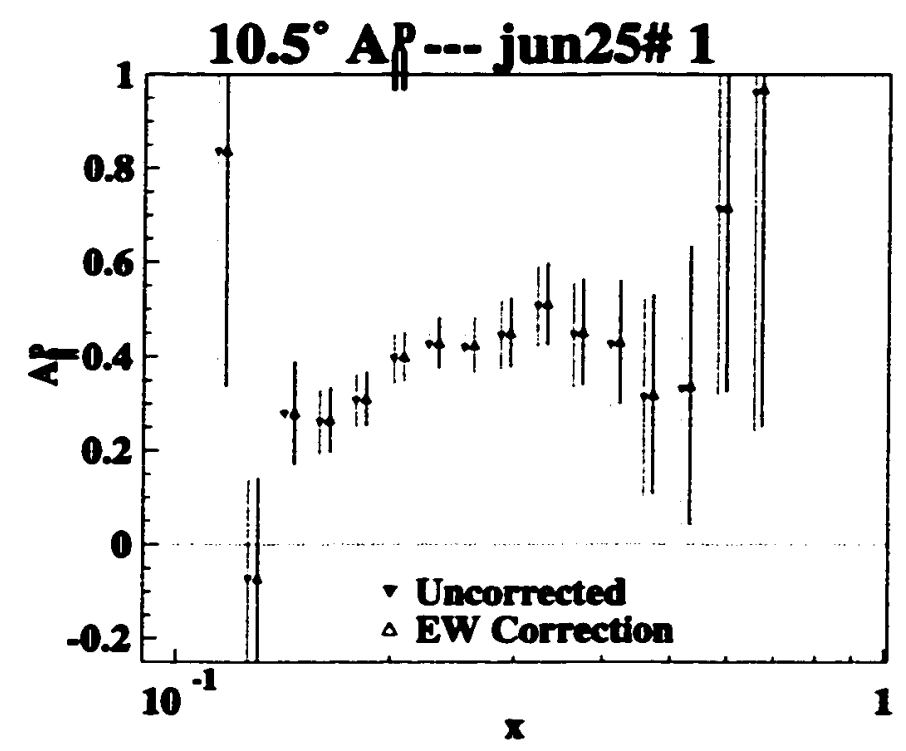

Figure 5.17: Comparison of asymmetry with and without EW correction.

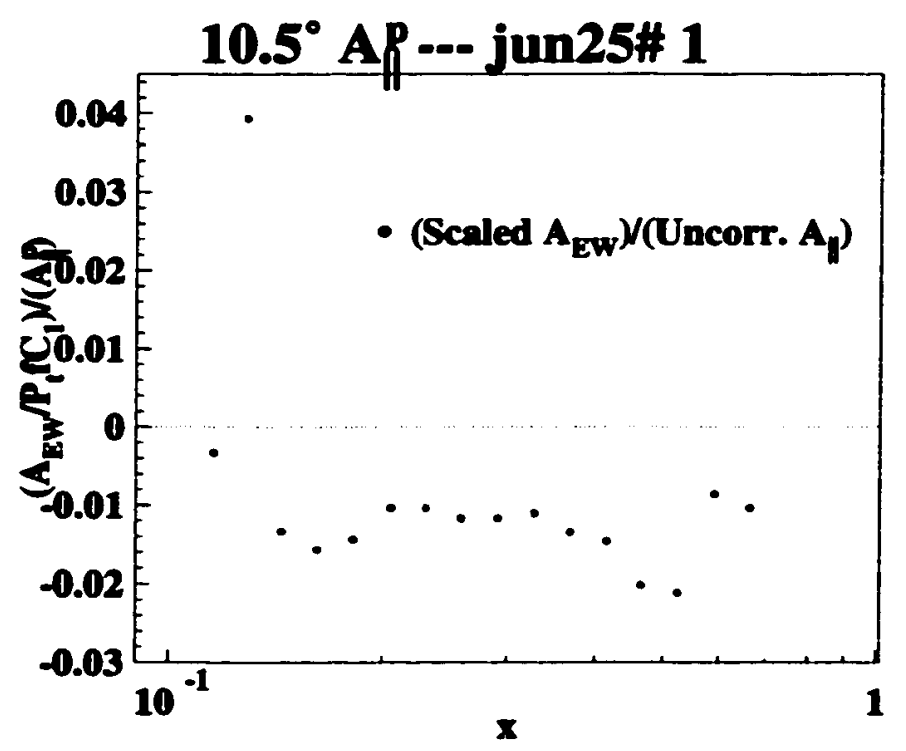

Figure 5.18: The difference between the proton asymmetry with and without the EW correction, quantity divided by the uncorrected asymmetry. This shows the percentage difference between the average scaled $A_{E W}$ for the proton dataset, and the uncorrected $A_{\| 1}$. The effect of $A_{E W}$ is to change $A_{\| l}$ by a few percent. 
the strange quark component, and the term $Y$ is given by

$$
Y=\frac{1-(1-y)^{2}}{1+(1-y)^{2}-y^{2} R\left(x, Q^{2}\right) /\left(1+R\left(x, Q^{2}\right)\right)},
$$

with $y=\nu / E$, and $R=\sigma_{L} / \sigma_{T}$ taking into account the longitudinal contributions of the $Z^{0}$ and photon exchange. We use the $R^{1990}$ [15] parameterization to generate $R\left(x, Q^{2}\right)$. The factor

$$
R_{s}(x)=\frac{2 s(x)}{u(x)+d(x)}
$$

is approximated by $R_{s}(x)=0.0$ for $x>0.2$, and $R_{s}(x)=0.5$ for $x<0.2$ [132]. Figure 5.17 shows the proton asymmetry with and without the electroweak asymmetry correction. Figure 5.18 shows the fractional change in the proton asymmetry due to the electroweak correction. The plotted quantity is $\left(A_{\text {corr. }}-A_{\text {uncorr. }}\right) / A_{\text {uncorr. }}$, where the $A_{\text {corr. }}$ and $A_{\text {uncorr. }}$ are the corrected and uncorrected asymmetries plotted in Fig. 5.17 .

\subsubsection{Corrections for Contaminant Events}

The electrons identified during the event analysis may include events which do not come from the deep inelastic electron scattering process. Some of these contaminant particles are pions which have been misidentified, others are pair produced electrons from the high-energy photons from $\pi^{0}$ decay. The measured physics asymmetry necessarily includes these events, but these events have no relevance to the DIS process, so should be removed if possible. The measured asymmetry is given by

$$
A^{\text {meas }}=\frac{\left(N_{e^{-}}^{L}+N_{e_{\bar{\gamma}}}^{L}+N_{\pi^{-}}^{L}\right)-\left(N_{e^{-}}^{R}+N_{e_{\bar{\gamma}}^{-}}^{R}+N_{\pi^{-}}^{R}\right)}{N_{e^{-}}^{L}+N_{e^{-}}^{R}+N_{e_{\bar{\gamma}}}^{L}+N_{e_{\bar{\gamma}}}^{R}+N_{\pi^{-}}^{L}+N_{\pi^{-}}^{R}}
$$

where the $N_{X}^{(L, R)}$ terms are the rates for each beam helicity for each species.

Since the contaminant events cannot be identified on an individual level, the corrections need to be made at the level of the physics result. Contaminant events 


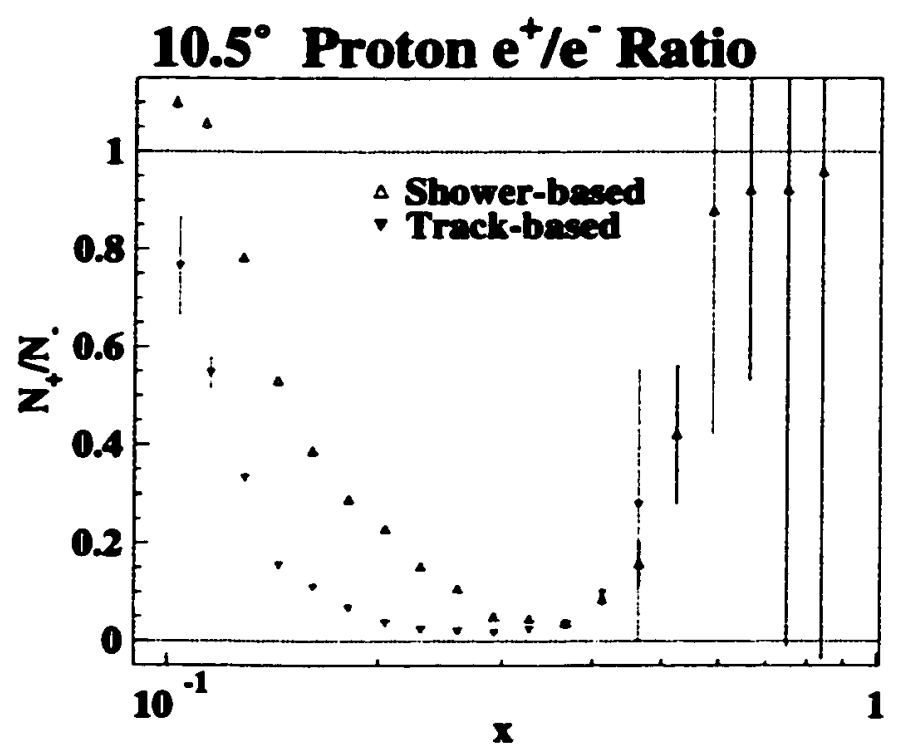

Figure 5.19: The ratio of positron rate to electron rate in the $10.5^{\circ}$ spectrometer for $t w o$ definitions. The shower-based definition has larger backgrounds due to fewer detector packages being required to form an event. Notice that for the two lowest bins the positron rate is distinctly higher than the electron rate for the shower-based definition; the reason for this is unknown. The track-based definition uses the calorimeter energy to determine the particle kinematics; notice that it does not extend beyond $x=0.46$ (Bin 33), because no positron events were detected in the higher bins.

could affect the physics asymmetry in two ways. The measured asymmetry may be rewritten in terms of the fractional event rates per species and the asymmetry of each species,

$$
\begin{aligned}
A^{\text {meas }} & =\frac{N_{e^{-}}}{N_{-}} \frac{\left(N_{e^{-}}^{L}-N_{e^{-}}^{R}\right)}{N_{e^{-}}}+\frac{N_{e_{\bar{\gamma}}}}{N_{-}} \frac{N_{e_{\bar{\gamma}}^{-}}^{L}-N_{e_{\bar{\gamma}}}^{R}}{N_{e_{\bar{\gamma}}}}+\frac{N_{\pi^{-}}}{N_{-}} \frac{N_{\pi^{-}}^{L}-N_{\pi^{-}}^{R}}{N_{\pi^{-}}} \\
& =\frac{N_{e^{-}}}{N_{-}} A^{D I S}+\frac{N_{e_{\bar{\gamma}}}}{N_{-}} A^{e_{\bar{\gamma}}^{-}}+\frac{N_{\pi^{-}}}{N_{-}} A^{\pi^{-}} \\
& =\alpha_{e^{-}} A^{D I S}+\alpha_{e_{\bar{\gamma}}} A^{e^{\bar{\gamma}}}+\alpha_{\pi_{-}} A^{\pi^{-}}
\end{aligned}
$$

where $N_{-}$is the total event rate, the other $N_{X}$ terms are the event rates for each species, $A^{D I S}$ is the asymmetry of the DIS electrons, the other $A^{X}$ terms are the asymmetries for the contaminant species, and the $\alpha$ terms are the fractional event rate for each species. Notice that $\alpha_{e^{-}}=1-\alpha_{e_{\bar{\gamma}}}-\alpha_{\pi^{-}}$.

If the contaminant events do not have an asymmetry, the second and third terms in Eq. 5.32 will not contribute. The measured asymmetry will be diluted by 
the ratio of the signal event rate to the total event rate, as compared to the true DIS asymmetry. If the contaminant events do have an asymmetry, then they would contribute to the measured asymmetry additively, in addition to the dilution. To correct the measured asymmetry in full generality, the asymmetry and fractional event rate for the contribution due to each contaminant species would need to be determined.

The pion contamination asymmetry can be determined simply by finding the asymmetry for one of the pion definitions, since the misidentified pions in the electron sample should have the same asymmetry as the correctly identified pions. The rate of contaminant pions in the electron sample is harder to extract. First, a parameterization of the pion E/P spectrum is found for each $x$ bin. This parameterization is scaled to fit the low $\mathrm{E} / \mathrm{P}$ spectrum in the electron definition. The magnitude of the model extending under the electron peak represents the size of the pion contamination in the electron sample.

The non-DIS electron asymmetry and rates are measured from the positron runs. The contaminant electrons are pair-produced, therefore their production is charge symmetric. However, the positron rate and asymmetries are themselves contaminated by positive pions. The same technique used to extract the negative pions from the DIS asymmetry is used to determine the clean positron asymmetry,

$$
\begin{aligned}
A^{e_{m e a s}^{+}} & =\frac{\left(N_{e_{\gamma}^{+}}^{L}+N_{\pi^{+}}^{L}\right)-\left(N_{e_{\gamma}^{+}}^{R}+N_{\pi^{+}}^{R}\right)}{N_{e_{\gamma}^{+}}^{L}+N_{e_{\gamma}^{+}}^{R}+N_{\pi^{+}}^{L}+N_{\pi^{+}}^{R}} \\
& =\frac{N_{e_{\gamma}^{+}}}{N_{+}} A^{e_{r}^{+}}+\frac{N_{\pi^{+}}}{N_{+}} A^{\pi^{+}} \\
& =\alpha_{e_{\gamma}^{+}} A^{e_{r}^{+}}+\alpha_{\pi^{+}} A^{\pi^{+}} .
\end{aligned}
$$

Then, since the production process is charge symmetric, $A^{e_{\gamma}^{+}}=A^{e_{\bar{\gamma}}^{-}}$, and

$$
A^{e_{\gamma}^{-}}=\frac{1}{\alpha_{e_{\gamma}^{+}}}\left(A^{e_{\text {meas }}^{+}}-\alpha_{\pi^{+}} A^{\pi^{+}}\right) .
$$




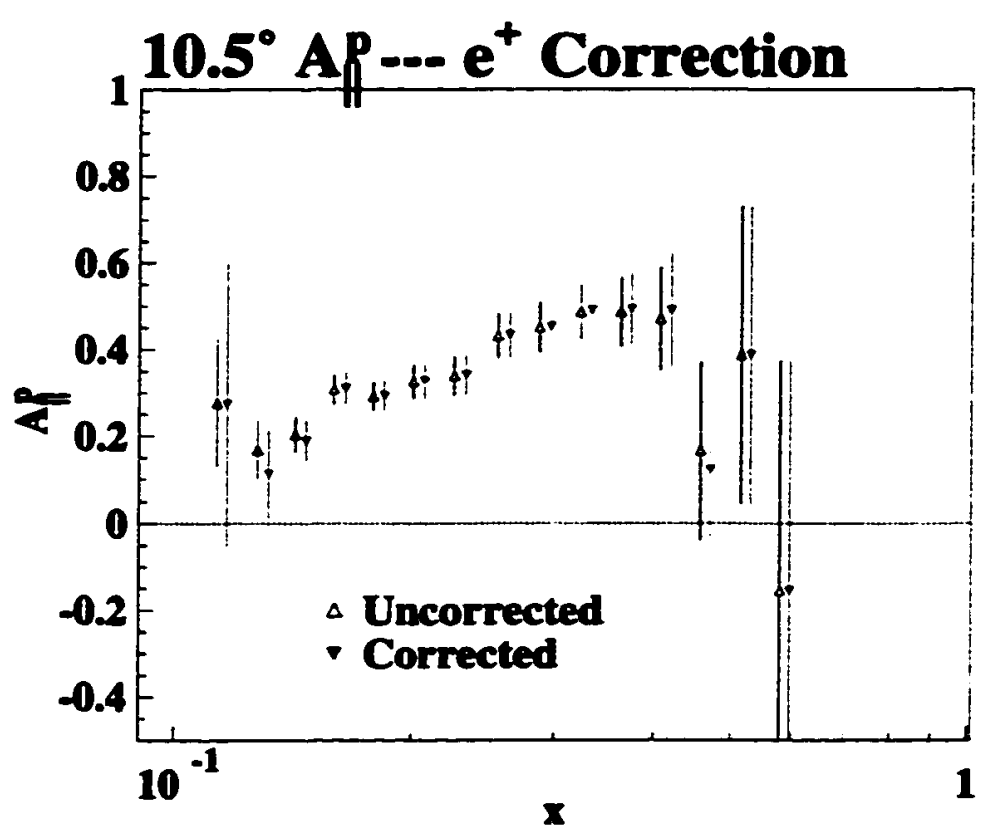

Figure 5.20: Comparison of asymmetry with and without pion/positron correction. Systematic errors are not shown. The two highest $x$ bins do not have a positron correction, because the positron definition did not have any events in those bins.

The electron contamination fractional rate is

$$
\alpha_{e_{\bar{\gamma}}}=\frac{N_{e_{\bar{\gamma}}}}{N_{-}}=\frac{N_{e_{\bar{\gamma}}}}{N_{+}} \frac{N_{+}}{N_{-}}=\frac{N_{+}}{N_{-}} \alpha_{e_{r}^{+}}
$$

So, the correction term for the pair-produced electrons is

$$
a_{e_{\bar{r}}} A^{e_{\bar{\gamma}}^{-}}=\frac{N_{+}}{N_{-}}\left(A^{e_{\text {meas }}^{+}}-\alpha_{\pi^{+}} A^{\pi^{+}}\right)
$$

Then the DIS asymmetry, corrected for the contaminations, is

$$
A^{D I S}=\frac{1}{1-\frac{N_{+}}{N_{-}}\left(1-\alpha_{\pi^{+}}\right)-\alpha_{\pi^{-}}}\left[A^{\text {meas }}-\frac{N_{+}}{N_{-}}\left(A^{\mathrm{emeas}^{+}}-\alpha_{\pi^{+}} A^{\pi^{+}}\right)-\alpha_{\pi^{-}} A^{\pi^{-}}\right]
$$

The statistical error is scaled by the term, $1 /\left(1-\alpha_{e-}-\alpha_{\pi^{-}}\right)$.

At this point, it should be stated that the above correction formula was used in full generality only for the $2.75^{\circ}$ and $5.5^{\circ}$ analyses. In the $10.5^{\circ}$ analysis, extracting a clean pion sample was very difficult. Accordingly, the $10.5^{\circ}$ analysis was done assuming that the positive and negative pion corrections are small and balance each 
other. The asymmetries and fractional rates for the pions were set to zero, and the correction formula simplified to

$$
A^{D I S}=\frac{1}{1-\frac{N_{+}}{N_{-}}}\left[A^{\text {meas }}-\frac{N_{+}}{N_{-}} A^{e_{\text {meas }}^{+}}\right]
$$

The effects of the positron correction for the $10.5^{\circ}$ spectrometer are shown in Fig. 5.20. The positron-to-electron ratio is shown in Fig. 5.19; the solid symbols in Fig. 5.19 correspond to the $N_{+} / N_{-}$rate for the definition shown in Fig. 5.20.

\subsubsection{Resolution Corrections}

The optics models of the spectrometers and a model of the asymmetry were used to estimate the effects on the asymmetry due to momentum and angle resolution in each spectrometer. A multiplicative correction was applied to the measured asymmetry; the correction was largest in the high $x$ bins, changing the asymmetry by a maximum of a $7 \%$ shift. In the $10.5^{\circ}$ spectrometer there are also large effects at low $x$. Across most of the $x$ range, the effect is less than about $2 \%[133,47]$.

\subsubsection{Radiative Correctioas}

The interpretation of the deep inelastic scattering asymmetry in terms of the structure functions, $F_{1}, g_{1}$, and $g_{2}$, assumes that the interaction of the electron and nucleon proceeds by one-photon exchange, and that the electron does not undergo any other interactions either before or after the DIS event. However, the actual DIS event includes higher order electromagnetic processes, and the electron may undergo bremsstrahlung either before or after the DIS event. An additional effect is that in undergoing external bremsstrahlung, the electron beam will lose some of the polarization, and so will produce a smaller measured asymmetry. The measured asymmetry is therefore radiatively smeared from the asymmetry resulting from the Born one-photon exchange. The radiative corrections serve to correct the measured asymmetry to generate the Born one-photon asymmetry. 


$\begin{array}{ccl}\text { Nucleon } & \text { Experiment } & \begin{array}{l}\text { Publication } \\ \text { Proton }\end{array} \\ \text { E155 } & \text { September 1998, E155 preliminary results; P. Bosted } \\ & \text { E143 } & \text { Phys. Rev. D58, 112003 (1998). } \\ & \text { E130 } & \text { Phys. Rev. Lett. 51, 1135 (1983). } \\ & \text { E80 } & \text { Phys. Rev. Lett. 41, 70 (1978). } \\ & \text { Hermes } & \text { Phys. Lett. B442, 484 (1998). } \\ & \text { SMC } & \text { Phys. Rev. D58, 112001 (1998). } \\ \text { Eeuteron } & \text { EMC } & \text { Nucl. Phys. B328, 1 (1989). } \\ & \text { E155 } & \text { September 1998, E155 preliminary results; P. Bosted } \\ & \text { E143 } & \text { Phys. Rev. D58, 112003 (1998). } \\ \text { Neutron } & \text { SMC } & \text { Phys. Rev. D58, 112001 (1998). } \\ & \text { E154 } & \text { Phys. Rev. Lett. 79, 26 (1997). } \\ & \text { E142 } & \text { Phys. Rev. D54, 6620 (1996). } \\ & \text { Hermes } & \text { Phys. Lett. B404, 383 (1997). }\end{array}$

Table 5.6: World data set used in the global fit to $A_{1}$ used in the radiative correction calculation. The data from E155, E154, E143, E130, and E80 were input to the fit as uncorrected data, and were corrected using the radiative corrections developed by this process in the subsequent iterations.

The E155 radiative corrections were developed by using an iterative fitting process to $A_{1}$, based upon the longitudinally polarized deep inelastic scattering world data set listed in Table 5.6 [134].

A simultaneous fit to the Born (radiatively corrected) asymmetry $A_{1}$ for the proton, deuteron and neutron was done using the set of functions,

$$
\begin{aligned}
& A_{1}^{p}\left(x, Q^{2}\right)=x^{\alpha}\left(a+b x+c x^{2}\right)\left(1+d / Q^{2}\right) \\
& A_{1}^{n}\left(x, Q^{2}\right)=x^{\beta}\left(a^{\prime}+b^{\prime} x+c^{\prime} x^{2}\right)\left(1+d^{\prime} / Q^{2}\right) \\
& A_{1}^{d}\left(x, Q^{2}\right)=\left(1-1.5 \omega_{D}\right) \frac{A_{1}^{p}\left(x, Q^{2}\right) F_{1}^{p}\left(x, Q^{2}\right)+A_{1}^{n}\left(x, Q^{2}\right) F_{1}^{n}\left(x, Q^{2}\right)}{2 F_{1}^{d}\left(x, Q^{2}\right)}
\end{aligned}
$$

where $F_{1}^{p}, F_{1}^{n}$, and $F_{1}^{d}$ are the respective unpolarized structure functions, and $\alpha, \beta$, $a, b, c, d, a^{\prime}, b^{\prime}, c^{\prime}$, and $d^{\prime}$ are the fit parameters. The fit is optimized by minimizing the combined chi-squared value, $\chi_{\text {total }}^{2}=\chi_{p}^{2}+\chi_{n}^{2}+\chi_{d}^{2}[134]$.

Once the fits of $A^{B o r n}$ have been produced, they are used as inputs to the RCSLACPOL ${ }^{1}$ program to calculate both internal and external radiative effects to

\footnotetext{
The RSLACPOL program takes as inputs: models for the polarized and unpolarized structure
} 


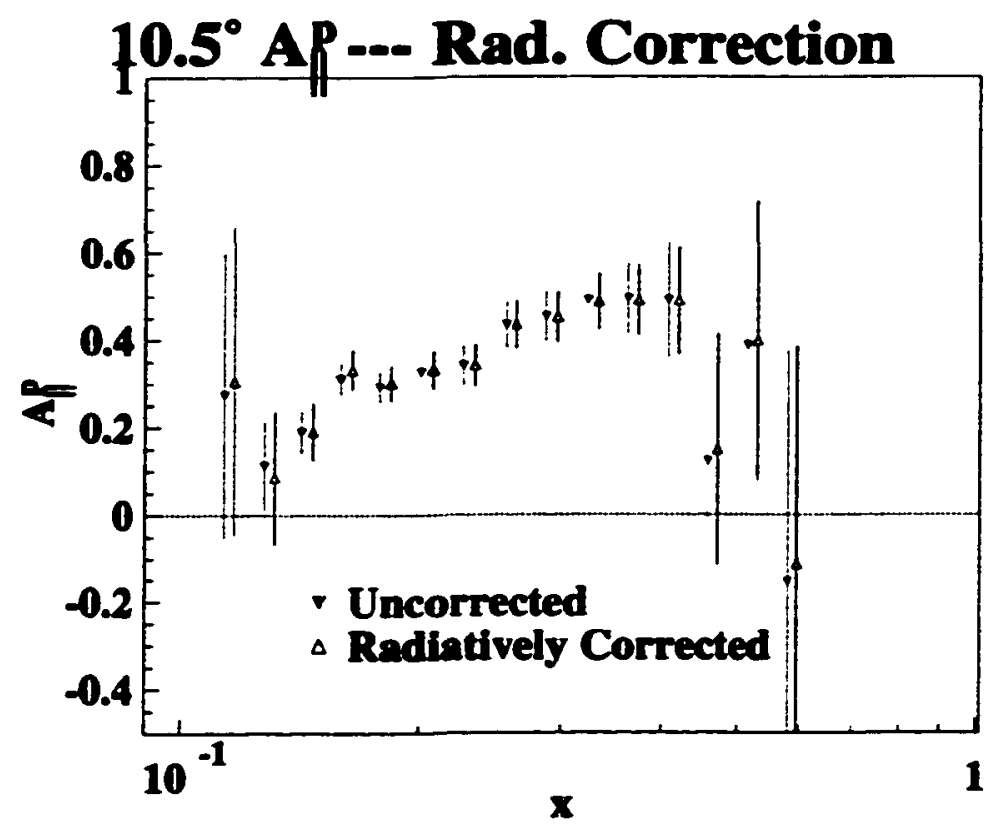

Figure 5.21: Comparison of asymmetry with and without radiative corrections. Systematic errors are not shown.

generate fits of $A^{\text {radiated, }}$ which would represent the measured asymmetries. Using the fits, $A_{f i t}^{B o r n}$ and $A_{f i t}^{\text {radiated }}$, a two part radiative correction is determined having the form,

$$
A^{B o r n}\left(x, Q^{2}\right)=\frac{A^{\text {radicted }}\left(x, Q^{2}\right)}{f_{R C}\left(x, Q^{2}\right)}+A_{R C}\left(x, Q^{2}\right)
$$

where $f_{R C}$ is a radiative dilution factor, and $A_{R C}$ is a radiative asymmetry [135].

\subsection{Fully Corrected Deep Inelastic Scattering Asymmetries, $A_{\|} \& A_{\perp}$}

The fully corrected parallel asymmetries for the proton and deuteron are shown in Fig. 5.22, and are tabulated in Tables 5.7, 5.8, and 5.9. These results use the dilution factor and nuclear corrections determined for each E155 target cell, the final proton polarization correction, and all other corrections. The determination of the systematic errors is discussed in Ref. [47] and Ref. [135] (systematic error of radiative corrections); however, the systematic error on the proton polarization for functions, the elastic nucleon form factors, the hydrogen and deuterium cross-sections, the structure function $R=\sigma_{L} / \sigma_{T}$, and the target model which contains the radiation lengths of all materials before and after the target [134]. 
the final proton polarization correction is $7 \%[136]$ rather than the value of $6 \%$ used in Ref. [47] and [135].

The fully corrected perpendicular asymmetries for the proton and deuteron are shown in Fig. 5.23 and tabulated in Tables 5.10, 5.11, and 5.12. The perpendicular asymmetry used the interim proton polarization correction [123], which used the relationship between ${ }^{15} \mathrm{~N}$ polarizations and proton polarizations to determine a mapping of measured polarization to true polarization. The $A_{\perp}$ measurements are statistics limited. 

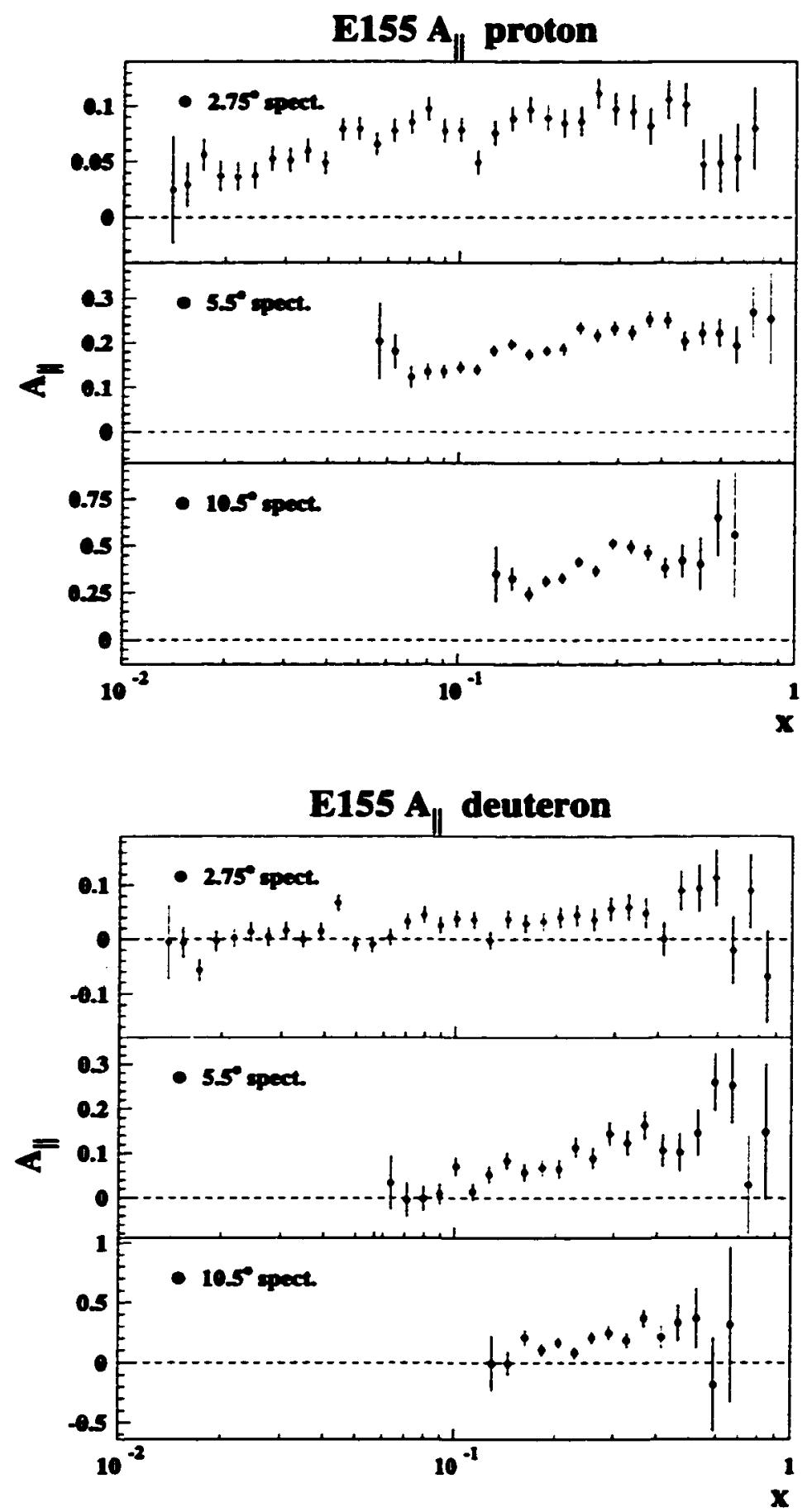

Figure 5.22: Fully corrected $A_{\|\|}^{p}$ and $A_{\|}^{d}$ for the three spectrometers at the measured $Q^{2}$. Error bars are statistical errors only. 
$\theta \approx 2.75^{\circ}$

\begin{tabular}{|c|c|c|c|c|c|}
\hline \multicolumn{3}{|r|}{ Proton } & \multicolumn{3}{|r|}{ Deuteron } \\
\hline$\langle x\rangle$ & $\left\langle Q^{2}\right\rangle$ & $A_{\|} \pm s t a t \pm s y s t$ & $\langle x\rangle$ & $\left\langle Q^{2}\right\rangle$ & $A_{\|} \pm$stat \pm syst \\
\hline 0.014 & 1.00 & $0.031 \pm 0.041 \pm 0.004$ & 0.014 & 1.00 & $-0.005 \pm 0.069 \pm 0.003$ \\
\hline 0.015 & 1.09 & $.039 \pm 0.016 \pm 0.004$ & 0.015 & 1.09 & $28 \pm 0.001$ \\
\hline 0.017 & 1.20 & $0.048 \pm 0.012 \pm 0.004$ & 0.017 & 1.20 & $-0.057 \pm 0$ \\
\hline 0.019 & 1.32 & $1 \pm 0.004$ & & 1.32 & $19 \pm 0.001$ \\
\hline 0.022 & 1.45 & $0.034 \pm 0.0$ & 0.022 & 1.45 & $0.003 \pm 0$ \\
\hline 0.024 & 1.59 & \pm 0.004 & 0.024 & 1.59 & $18 \pm 0.001$ \\
\hline 0.027 & 1.73 & $9 \pm 0.004$ & 0.027 & 1.73 & $0.005 \pm 0$ \\
\hline 0.031 & & 04 & & 1.88 & $17 \pm 0.001$ \\
\hline 0.035 & 2.04 & $9 \pm 0.004$ & 0.035 & 2.04 & $0.000 \pm 0$ \\
\hline 39 & 2.22 & \pm 0.005 & & 2.21 & \pm 0.001 \\
\hline 0.044 & 2.40 & $8 \pm 0.005$ & 0.044 & 2.39 & \pm 0.001 \\
\hline 49 & 2.59 & \pm 0.005 & 0.049 & 2.58 & \pm 0.001 \\
\hline 0.056 & 2.78 & \pm 0.005 & 0.056 & 2.77 & $=0.001$ \\
\hline 63 & 2.97 & & 0.063 & 2.97 & $=0.001$ \\
\hline 0.071 & 3.17 & \pm 0.005 & 0.071 & 3.17 & $=0.001$ \\
\hline 79 & 3.37 & & & 3.36 & 0.001 \\
\hline 0.089 & 3.57 & 36 & 0.089 & 3.57 & $=0.001$ \\
\hline 01 & 3.77 & & 0.101 & 3.77 & $=0.001$ \\
\hline 0.113 & 3.97 & \pm 0.006 & 0.113 & 3.97 & $=0.001$ \\
\hline 27 & 4.17 & & 0.128 & 4.17 & $=0.002$ \\
\hline 0.144 & 4.36 & 06 & 0.144 & 4.36 & $=0.002$ \\
\hline 0.162 & 4.54 & .006 & 0.162 & 4.55 & 0.002 \\
\hline 0.182 & 4.72 & \pm 0.0 & 0.182 & 4.72 & 0.002 \\
\hline 0.205 & 4.88 & \pm 0.007 & 0.205 & 4.89 & .002 \\
\hline 0.230 & 5.04 & 07 & & 5.04 & .002 \\
\hline 0.259 & 5.18 & \pm 0.007 & 0.259 & 5.19 & .002 \\
\hline 0.292 & 5.33 & 07 & & 5.33 & .002 \\
\hline 0.328 & 5.47 & \pm 0.007 & 0.328 & 5.47 & .003 \\
\hline 0.370 & 5.57 & \pm 0.007 & 0.370 & 5.58 & 0.003 \\
\hline 0.416 & 5.68 & 0.0 & 0.416 & 5.68 & 0.003 \\
\hline 0.468 & 5.78 & $7 \pm 0.007$ & & 5.78 & $=0.003$ \\
\hline 0.527 & 5.87 & $0 \pm 0.006$ & 0.527 & 5.87 & 0.003 \\
\hline 0.593 & 5.94 & $3 \pm 0.006$ & 0.593 & 5.95 & $53 \pm 0.003$ \\
\hline 0.667 & 6.00 & & & 6.00 & $62 \pm 0.003$ \\
\hline 0.751 & 6.06 & $0.077 \pm 0.032 \pm 0.006$ & 0.751 & 6.07 & $0.089 \pm 0.069 \pm 0.003$ \\
\hline 0.846 & 6.12 & $0.129 \pm 0.045 \pm 0.005$ & 0.846 & 6.12 & $-0.069 \pm 0.085 \pm 0.003$ \\
\hline
\end{tabular}

Table 5.7: Final $A_{\|}^{p}$ and $A_{\|}^{d}$ for the $2.75^{\circ}$ spectrometer. $Q^{2}$ is in $(\mathrm{GeV} / c)^{2}$. 
$\theta \approx 5.5^{\circ}$

\begin{tabular}{|ccc|ccc|}
\hline \multicolumn{3}{c}{ Proton } & \multicolumn{3}{c}{ Deuteron } \\
\hline$\langle x\rangle$ & $\left\langle Q^{2}\right\rangle$ & $A_{\|} \pm$stat \pm syst & $\langle x\rangle$ & $\left\langle Q^{2}\right\rangle$ & $A_{\|} \pm$stat $\pm s y s t$ \\
\hline 0.057 & 4.01 & $0.192 \pm 0.072 \pm 0.008$ & 0.057 & 4.00 & $-0.215 \pm 0.140 \pm 0.002$ \\
0.063 & 4.39 & $0.146 \pm 0.032 \pm 0.009$ & 0.063 & 4.39 & $0.034 \pm 0.061 \pm 0.002$ \\
0.071 & 4.83 & $0.106 \pm 0.021 \pm 0.009$ & 0.071 & 4.83 & $-0.004 \pm 0.038 \pm 0.002$ \\
0.080 & 5.30 & $0.116 \pm 0.016 \pm 0.010$ & 0.080 & 5.30 & $-0.002 \pm 0.028 \pm 0.002$ \\
0.090 & 5.80 & $0.123 \pm 0.013 \pm 0.010$ & 0.090 & 5.80 & $0.010 \pm 0.023 \pm 0.003$ \\
0.101 & 6.32 & $0.129 \pm 0.012 \pm 0.011$ & 0.101 & 6.32 & $0.071 \pm 0.021 \pm 0.003$ \\
0.113 & 6.87 & $0.124 \pm 0.011 \pm 0.012$ & 0.113 & 6.87 & $0.014 \pm 0.020 \pm 0.003$ \\
0.128 & 7.43 & $0.169 \pm 0.010 \pm 0.012$ & 0.128 & 7.43 & $0.053 \pm 0.019 \pm 0.003$ \\
0.144 & 8.02 & $0.167 \pm 0.010 \pm 0.013$ & 0.144 & 8.02 & $0.084 \pm 0.019 \pm 0.004$ \\
0.162 & 8.62 & $0.150 \pm 0.011 \pm 0.014$ & 0.162 & 8.62 & $0.057 \pm 0.020 \pm 0.004$ \\
0.182 & 9.23 & $0.166 \pm 0.011 \pm 0.014$ & 0.182 & 9.23 & $0.068 \pm 0.021 \pm 0.004$ \\
0.205 & 9.85 & $0.164 \pm 0.012 \pm 0.015$ & 0.205 & 9.85 & $0.065 \pm 0.021 \pm 0.005$ \\
0.230 & 10.48 & $0.207 \pm 0.012 \pm 0.016$ & 0.230 & 10.48 & $0.114 \pm 0.023 \pm 0.005$ \\
0.259 & 11.11 & $0.195 \pm 0.013 \pm 0.016$ & 0.259 & 11.11 & $0.089 \pm 0.024 \pm 0.006$ \\
0.292 & 11.73 & $0.211 \pm 0.013 \pm 0.017$ & 0.292 & 11.73 & $0.145 \pm 0.026 \pm 0.006$ \\
0.328 & 12.34 & $0.203 \pm 0.014 \pm 0.017$ & 0.328 & 12.34 & $0.123 \pm 0.028 \pm 0.007$ \\
0.370 & 12.94 & $0.228 \pm 0.015 \pm 0.017$ & 0.370 & 12.94 & $0.163 \pm 0.032 \pm 0.00 \tau$ \\
0.416 & 13.52 & $0.233 \pm 0.016 \pm 0.017$ & 0.416 & 13.53 & $0.106 \pm 0.036 \pm 0.007$ \\
0.468 & 14.08 & $0.181 \pm 0.018 \pm 0.017$ & 0.468 & 14.09 & $0.103 \pm 0.043 \pm 0.007$ \\
0.526 & 14.61 & $0.208 \pm 0.022 \pm 0.017$ & 0.526 & 14.63 & $0.146 \pm 0.052 \pm 0.008$ \\
0.592 & 15.11 & $0.204 \pm 0.028 \pm 0.016$ & 0.592 & 15.12 & $0.259 \pm 0.065 \pm 0.008$ \\
0.666 & 15.58 & $0.172 \pm 0.036 \pm 0.015$ & 0.666 & 15.59 & $0.252 \pm 0.085 \pm 0.007$ \\
0.749 & 15.99 & $0.231 \pm 0.048 \pm 0.013$ & 0.749 & 16.00 & $0.030 \pm 0.111 \pm 0.007$ \\
0.843 & 16.34 & $0.193 \pm 0.077 \pm 0.011$ & 0.843 & 16.35 & $0.148 \pm 0.153 \pm 0.006$ \\
\hline \hline
\end{tabular}

Table 5.8: Final $A_{\|}^{p}$ and $A_{\|}^{d}$ for the $5.5^{\circ}$ spectrometer. $Q^{2}$ is in $(\mathrm{GeV} / \mathrm{c})^{2}$. 
$\theta \approx 10.5^{\circ}$

\begin{tabular}{|ccc|ccc|}
\hline \hline \multicolumn{3}{c}{ Proton } & \multicolumn{3}{c|}{ Deuteron } \\
\hline$\langle x\rangle$ & $\left\langle Q^{2}\right\rangle$ & $A_{\|} \pm$stat \pm syst & $\langle x\rangle$ & $\left\langle Q^{2}\right\rangle$ & $A_{\|} \pm$stat \pm syst \\
\hline 0.130 & 10.04 & $0.333 \pm 0.125 \pm 0.017$ & 0.130 & 10.04 & $-0.004 \pm 0.228 \pm 0.005$ \\
0.145 & 11.14 & $0.303 \pm 0.051 \pm 0.019$ & 0.145 & 11.14 & $-0.006 \pm 0.096 \pm 0.006$ \\
0.162 & 12.35 & $0.251 \pm 0.033 \pm 0.020$ & 0.163 & 12.37 & $0.207 \pm 0.063 \pm 0.006$ \\
0.182 & 13.70 & $0.299 \pm 0.026 \pm 0.022$ & 0.182 & 13.70 & $0.107 \pm 0.049 \pm 0.007$ \\
0.205 & 15.13 & $0.299 \pm 0.023 \pm 0.023$ & 0.205 & 15.15 & $0.166 \pm 0.046 \pm 0.008$ \\
0.230 & 16.66 & $0.349 \pm 0.024 \pm 0.025$ & 0.230 & 16.67 & $0.086 \pm 0.047 \pm 0.009$ \\
0.259 & 18.28 & $0.326 \pm 0.026 \pm 0.027$ & 0.259 & 18.28 & $0.206 \pm 0.051 \pm 0.010$ \\
0.291 & 20.03 & $0.447 \pm 0.030 \pm 0.029$ & 0.292 & 20.04 & $0.249 \pm 0.057 \pm 0.011$ \\
0.328 & 21.86 & $0.433 \pm 0.032 \pm 0.031$ & 0.328 & 21.86 & $0.186 \pm 0.062 \pm 0.012$ \\
0.369 & 23.80 & $0.413 \pm 0.035 \pm 0.033$ & 0.369 & 23.78 & $0.370 \pm 0.073 \pm 0.013$ \\
0.413 & 25.85 & $0.365 \pm 0.046 \pm 0.034$ & 0.414 & 25.90 & $0.211 \pm 0.098 \pm 0.015$ \\
0.465 & 27.94 & $0.410 \pm 0.075 \pm 0.036$ & 0.465 & 28.04 & $0.332 \pm 0.153 \pm 0.016$ \\
0.524 & 30.12 & $0.318 \pm 0.122 \pm 0.036$ & 0.524 & 30.19 & $0.366 \pm 0.253 \pm 0.017$ \\
0.590 & 32.30 & $0.642 \pm 0.176 \pm 0.036$ & 0.590 & 32.36 & $-0.183 \pm 0.389 \pm 0.017$ \\
0.663 & 34.72 & $0.417 \pm 0.293 \pm 0.035$ & 0.661 & 34.79 & $0.315 \pm 0.649 \pm 0.017$ \\
\hline \hline
\end{tabular}

Table 5.9: Final $A_{\|\|}^{p}$ and $A_{\|}^{d}$ for the $10.5^{\circ}$ spectrometer. $Q^{2}$ is in $(\mathrm{GeV} / \mathrm{c})^{2}$. 

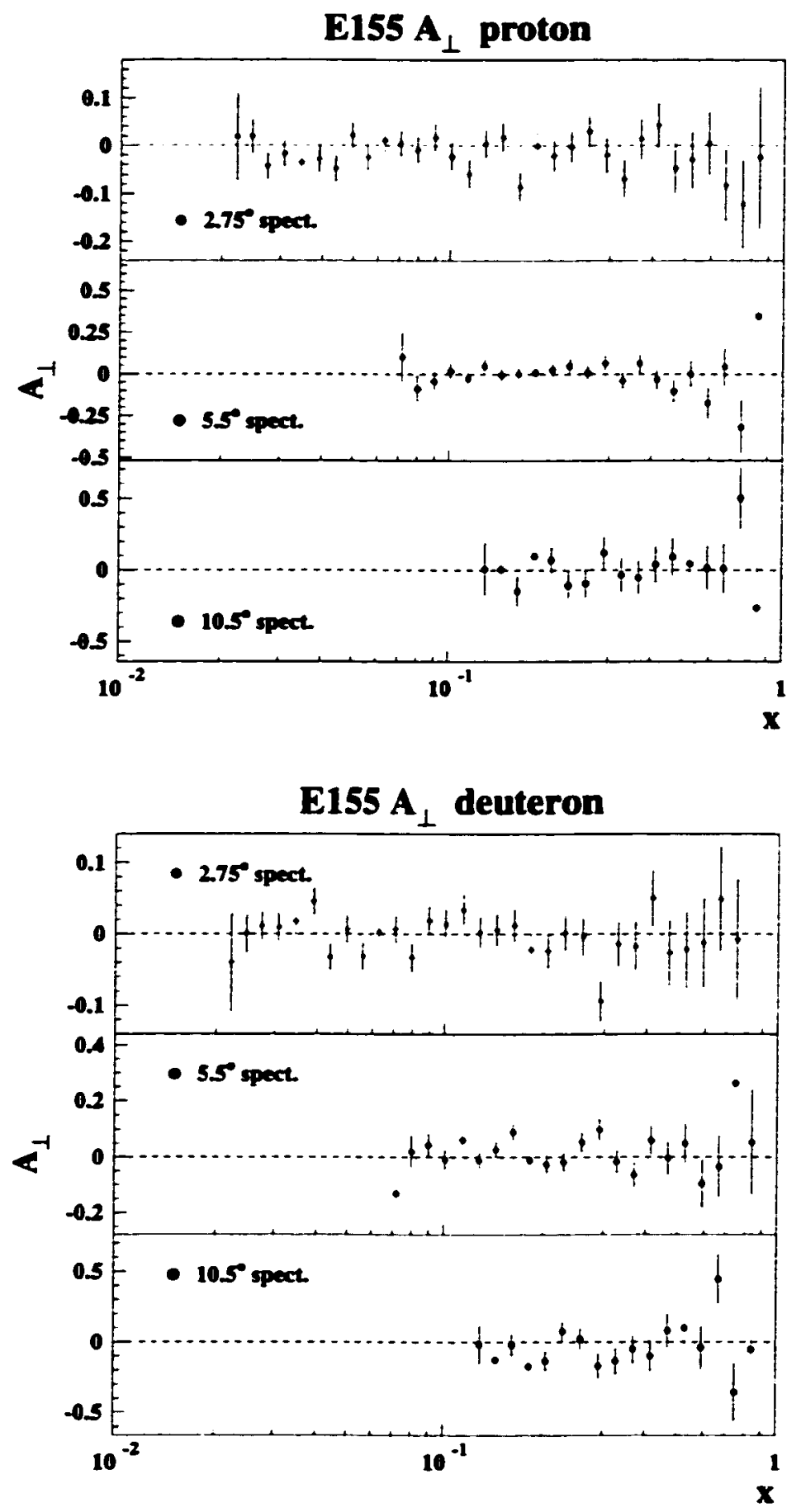

Figure 5.23: Fully corrected $A_{\perp}^{p}$ and $A_{\perp}^{d}$ for the three spectrometers at the measured $Q^{2}$. Error bars are statistical errors only. 
$\theta \approx 2.75^{\circ}$

\begin{tabular}{|ccc|ccc|}
\hline \multicolumn{6}{c}{ Proton } \\
\hline$\langle x\rangle$ & $\left\langle Q^{2}\right\rangle$ & \multicolumn{1}{c}{$A_{\perp} \pm$ stat } & $\langle x\rangle$ & $\left\langle Q^{2}\right\rangle$ & \multicolumn{1}{c}{$A_{\perp} \pm$ stat } \\
\hline 0.022 & 1.15 & $0.019 \pm 0.092$ & 0.022 & 1.15 & $-0.040 \pm 0.069$ \\
0.025 & 1.25 & $0.020 \pm 0.037$ & 0.025 & 1.25 & $0.001 \pm 0.028$ \\
0.027 & 1.35 & $-0.043 \pm 0.027$ & 0.027 & 1.35 & $0.011 \pm 0.020$ \\
0.031 & 1.42 & $-0.017 \pm 0.028$ & 0.031 & 1.42 & $0.010 \pm 0.020$ \\
0.035 & 1.48 & $-0.035 \pm 0.028$ & 0.035 & 1.48 & $0.018 \pm 0.020$ \\
0.039 & 1.56 & $-0.029 \pm 0.028$ & 0.039 & 1.56 & $0.046 \pm 0.020$ \\
0.044 & 1.65 & $-0.048 \pm 0.027$ & 0.044 & 1.65 & $-0.032 \pm 0.020$ \\
0.049 & 1.76 & $0.022 \pm 0.027$ & 0.049 & 1.76 & $0.006 \pm 0.019$ \\
0.056 & 1.86 & $-0.024 \pm 0.028$ & 0.056 & 1.86 & $-0.031 \pm 0.020$ \\
0.063 & 1.95 & $0.011 \pm 0.028$ & 0.063 & 1.95 & $0.003 \pm 0.020$ \\
0.071 & 2.05 & $0.005 \pm 0.028$ & 0.071 & 2.05 & $0.007 \pm 0.020$ \\
0.079 & 2.16 & $-0.008 \pm 0.028$ & 0.079 & 2.16 & $-0.033 \pm 0.020$ \\
0.089 & 2.26 & $0.017 \pm 0.029$ & 0.089 & 2.26 & $0.019 \pm 0.021$ \\
0.101 & 2.35 & $-0.023 \pm 0.029$ & 0.101 & 2.3 .5 & $0.014 \pm 0.021$ \\
0.113 & 2.45 & $-0.058 \pm 0.030$ & 0.113 & 2.45 & $0.034 \pm 0.021$ \\
0.128 & 2.5 .5 & $0.004 \pm 0.030$ & 0.128 & 2.55 & $0.002 \pm 0.022$ \\
0.144 & 2.64 & $0.018 \pm 0.031$ & 0.144 & 2.64 & $0.006 \pm 0.023$ \\
0.162 & 2.72 & $-0.085 \pm 0.031$ & 0.162 & 2.72 & $0.013 \pm 0.023$ \\
0.182 & 2.80 & $-0.001 \pm 0.031$ & 0.182 & 2.80 & $-0.021 \pm 0.024$ \\
0.205 & 2.88 & $-0.021 \pm 0.032$ & 0.205 & 2.88 & $-0.024 \pm 0.024$ \\
0.230 & 2.96 & $-0.002 \pm 0.033$ & 0.230 & 2.96 & $0.001 \pm 0.025$ \\
0.259 & 3.03 & $0.030 \pm 0.034$ & 0.259 & 3.03 & $-0.004 \pm 0.027$ \\
0.292 & 3.09 & $-0.019 \pm 0.037$ & 0.292 & 3.09 & $-0.094 \pm 0.029$ \\
0.328 & 3.15 & $-0.069 \pm 0.040$ & 0.328 & 3.15 & $-0.014 \pm 0.032$ \\
0.370 & 3.20 & $0.014 \pm 0.043$ & 0.370 & 3.21 & $-0.018 \pm 0.035$ \\
0.416 & 3.26 & $0.043 \pm 0.047$ & 0.416 & 3.26 & $0.050 \pm 0.040$ \\
0.468 & 3.29 & $-0.047 \pm 0.053$ & 0.468 & 3.29 & $-0.027 \pm 0.046$ \\
0.527 & 3.33 & $-0.029 \pm 0.059$ & 0.527 & 3.33 & $-0.022 \pm 0.054$ \\
0.593 & 3.36 & $0.005 \pm 0.066$ & 0.593 & 3.35 & $-0.012 \pm 0.063$ \\
0.667 & 3.38 & $-0.082 \pm 0.075$ & 0.668 & 3.38 & $0.049 \pm 0.074$ \\
0.752 & 3.40 & $-0.122 \pm 0.093$ & 0.751 & 3.40 & $-0.008 \pm 0.085$ \\
0.846 & 3.43 & $-0.024 \pm 0.150$ & 0.846 & 3.42 & $-0.349 \pm 0.105$ \\
\hline \hline
\end{tabular}

Table 5.10: Final $A_{\perp}^{p}$ and $A_{\perp}^{d}$ for the $2.75^{\circ}$ spectrometer. $Q^{2}$ is in $(\mathrm{GeV} / \mathrm{c})^{2}$. 
$\theta \approx 5.5^{\circ}$

\begin{tabular}{|crr|rrr|}
\hline \multicolumn{4}{c}{ Proton } & \multicolumn{3}{c}{ Deuteron } \\
\hline$\langle x\rangle$ & $\left\langle Q^{2}\right\rangle$ & \multicolumn{1}{c}{$A_{\perp} \pm$ stat } & $\langle x\rangle$ & $\left\langle Q^{2}\right\rangle$ & \multicolumn{1}{c|}{$A_{\perp} \pm$ stat } \\
\hline 0.072 & 3.71 & $0.102 \pm 0.149$ & $\mathbf{0 . 0 7 2}$ & 3.71 & $-0.131 \pm 0.108$ \\
0.080 & 4.03 & $-0.088 \pm 0.080$ & $\mathbf{0 . 0 8 0}$ & 4.03 & $0.021 \pm 0.058$ \\
0.090 & 4.39 & $-0.041 \pm 0.058$ & $\mathbf{0 . 0 9 0}$ & 4.39 & $0.044 \pm 0.042$ \\
0.101 & 4.76 & $0.020 \pm 0.048$ & $\mathbf{0 . 1 0 1}$ & 4.76 & $-0.009 \pm 0.035$ \\
0.113 & 5.14 & $-0.028 \pm 0.043$ & 0.113 & 5.13 & $0.062 \pm 0.032$ \\
0.128 & 5.52 & $0.050 \pm 0.040$ & 0.128 & 5.52 & $-0.011 \pm 0.030$ \\
0.144 & 5.91 & $-0.006 \pm 0.039$ & 0.144 & 5.91 & $0.028 \pm 0.029$ \\
0.162 & 6.31 & $0.002 \pm 0.039$ & 0.162 & 6.31 & $0.090 \pm 0.029$ \\
0.182 & 6.69 & $0.011 \pm 0.039$ & 0.182 & 6.69 & $-0.011 \pm 0.030$ \\
0.205 & 7.07 & $0.026 \pm 0.041$ & 0.205 & 7.07 & $-0.026 \pm 0.032$ \\
0.230 & 7.45 & $0.051 \pm 0.042$ & 0.230 & 7.45 & $-0.018 \pm 0.034$ \\
0.259 & 7.83 & $0.009 \pm 0.045$ & 0.259 & 7.83 & $0.054 \pm 0.036$ \\
0.292 & 8.19 & $0.063 \pm 0.047$ & 0.292 & 8.19 & $0.098 \pm 0.039$ \\
0.328 & 8.53 & $-0.037 \pm 0.051$ & 0.329 & 8.53 & $-0.016 \pm 0.042$ \\
0.370 & 8.89 & $0.064 \pm 0.055$ & 0.370 & 8.88 & $-0.065 \pm 0.047$ \\
0.416 & 9.23 & $-0.032 \pm 0.061$ & 0.416 & 9.23 & $0.061 \pm 0.053$ \\
0.468 & 9.54 & $-0.104 \pm 0.070$ & 0.468 & 9.54 & $-0.004 \pm 0.061$ \\
0.526 & 9.82 & $0.001 \pm 0.080$ & 0.527 & 9.82 & $0.049 \pm 0.074$ \\
0.593 & 10.08 & $-0.174 \pm 0.095$ & 0.592 & 10.08 & $-0.095 \pm 0.089$ \\
0.666 & 10.35 & $0.045 \pm 0.117$ & 0.666 & 10.36 & $-0.034 \pm 0.112$ \\
0.750 & 10.59 & $-0.316 \pm 0.158$ & 0.749 & 10.57 & $0.263 \pm 0.143$ \\
0.843 & 10.79 & $0.348 \pm 0.268$ & 0.843 & 10.79 & $0.052 \pm 0.159$ \\
\hline \hline
\end{tabular}

Table 5.11: Final $A_{\perp}^{p}$ and $A_{\perp}^{d}$ for the $5.5^{\circ}$ spectrometer. $Q^{2}$ is in $(\mathrm{GeV} / \mathrm{c})^{2}$. 
$\theta \approx 10.5^{\circ}$

\begin{tabular}{|c|c|c|c|c|c|}
\hline \multicolumn{3}{|c|}{ Proton } & \multicolumn{3}{|c|}{ Deuteron } \\
\hline$\langle x\rangle$ & $\left\langle Q^{2}\right\rangle$ & $A_{\perp} \pm s t a t$ & $\langle x\rangle$ & $\left\langle Q^{2}\right\rangle$ & $A_{\perp} \pm$ stat \\
\hline 0.129 & 7.58 & $0.009 \pm 0.186$ & 0.129 & 7.58 & $-0.018 \pm 0.139$ \\
\hline 0.144 & 8.38 & $0.007 \pm 0.120$ & 0.144 & 8.38 & $-0.128 \pm 0.090$ \\
\hline 0.162 & 9.27 & $-0.145 \pm 0.106$ & 0.162 & $9.2 \pi$ & $-0.023 \pm 0.079$ \\
\hline 0.182 & 10.32 & $0.100 \pm 0.098$ & 0.182 & 10.33 & $-0.173 \pm 0.07 .5$ \\
\hline 0.20 .5 & 11.44 & $0.072 \pm 0.093$ & 0.205 & 11.46 & $-0.136 \pm 0.070$ \\
\hline 0.230 & 12.62 & $-0.105 \pm 0.095$ & 0.230 & 12.63 & $0.077 \pm 0.074$ \\
\hline 0.259 & 13.78 & $-0.090 \pm 0.103$ & 0.259 & 13.77 & $0.022 \pm 0.080$ \\
\hline 0.292 & 14.97 & $0.121 \pm 0.114$ & 0.292 & 14.94 & $-0.172 \pm 0.090$ \\
\hline 0.328 & 16.14 & $-0.033 \pm 0.119$ & 0.329 & 16.09 & $-0.136 \pm 0.097$ \\
\hline 0.370 & 17.23 & $-0.054 \pm 0.121$ & 0.370 & 17.23 & $-0.051 \pm 0.102$ \\
\hline 0.416 & 18.43 & $0.041 \pm 0.130$ & 0.417 & 18.42 & $-0.100 \pm 0.111$ \\
\hline 0.468 & 19.44 & $0.092 \pm 0.135$ & 0.469 & 19.47 & $0.080 \pm 0.119$ \\
\hline 0.528 & 20.44 & $0.044 \pm 0.144$ & 0.527 & 20.49 & $0.098 \pm 0.136$ \\
\hline 0.593 & 21.49 & $0.014 \pm 0.158$ & 0.593 & 21.59 & $-0.040 \pm 0.156$ \\
\hline 0.668 & 22.64 & $0.012 \pm 0.177$ & 0.667 & 22.73 & $0.448 \pm 0.179$ \\
\hline 0.750 & 23.72 & $0.503 \pm 0.217$ & 0.750 & 23.75 & $-0.356 \pm 0.209$ \\
\hline 0.84 .5 & 24.84 & $-0.264 \pm 0.327$ & 0.845 & 24.94 & $-0.053 \pm 0.241$ \\
\hline
\end{tabular}

Table 5.12: Final $A_{\perp}^{p}$ and $A_{\perp}^{d}$ for the $10.5^{\circ}$ spectrometer. $Q^{2}$ is in $(\mathrm{GeV} / \mathrm{c})^{2}$. 


\section{CHAPTER 6}

\section{RESULTS}

The fully-corrected deep inelastic scattering asymmetries were presented in Section 5.7. These asymmetries may now be used to extract the structure functions for the proton and deuteron. and also may be used to determine the asymmetries and structure functions for the neutron.

Normally, as presented in Section 1.3, the measured asymmetries. $A_{\| \mid}\left(x \cdot Q^{2}\right)$ and $\mathcal{A}_{\perp}\left(x, Q^{2}\right)$, are used to determine the photo-absorption asymmetries and the spin structure functions. However, the equations presented in that section require that $A_{\|}$and $A_{\perp}$ be measured at the same kinematics; since E 1.55 used different beam energies for the $A_{\|}$and $A_{\perp}$ measurements, the kinematics differ. By using a model of $g_{2}\left(x, Q^{2}\right) . A_{1}$ and $g_{1}$ may be extracted from the measured $A_{\|}$. Similarly: a model of $A_{1}\left(x . Q^{2}\right)$ is used to extract $A_{2}$ and $g_{2}$ from $A_{\perp}$.

\subsection{Virtual Photon Asymmetries, $A_{1} \& A_{2}$}

As was mentioned in Section 5.6.4. a global fit of the virtual photon asymmetry. $A_{1}$, was done as part of the radiative correction process. This fit included $A_{1}$ data points from the preliminary E15.5 analysis.

Starting from Eq. 1.4i, and using some of the formalism presented in Section 1.3. $A_{1}$ may be expressed in terms of $A_{\|}$and $g_{2}$ [28]. When $g_{2}$ is assumed to be given by $g_{2}^{W W}$ (which assumption is consistent with experimental data on $g_{2}$ up to and including E155 [42]),

$$
A_{1}=\frac{A_{\|}}{D^{\prime}}\left[1+\frac{x M y}{E+E^{\prime} \cos \theta}\right]-\frac{g_{2}^{W W}}{F_{1}}\left[\frac{4 x M E^{\prime} \cos ^{2} \frac{\theta}{2}}{\nu\left(E+E^{\prime} \cos \theta\right)}\right] .
$$




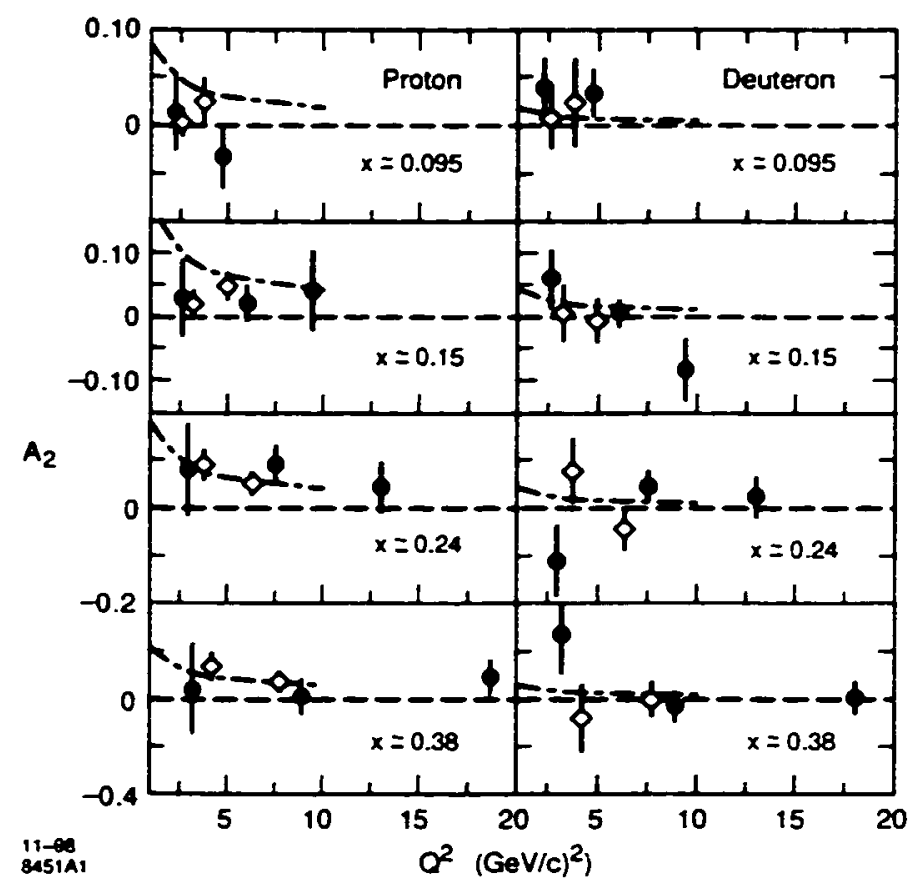

Figure 6.1: Plot of E155 $A_{2}$ (filled circles) for the proton and deuteron vs $Q^{2}$ for selected bins in $x$. Also shown are data (open diamonds) from E143 [28] and the bag model calculation by Stratmann [137]. Figure from [42].

where the variables are as described in the discussion of formalism in Section 1.3. Alternately, if $g_{2}=0$ is assumed, $A_{1}$ can be described entirely in terms of $A_{\|}$.

The E1.5.5 $A_{1}$ data will be presented in Section 7.4 in the context of using the global fit to discuss $Q^{2}$ dependence of the structure functions. Tables 6.1 and 6.2 include the final collaboration result of $g_{1} / F_{1}$ for the three spectrometers, which is approximately equal to $A_{1}$. Both $A_{1}$ and $g_{1} / F_{1}$ are consistent with being independent of $Q^{2}$, but are more consistent with having a mild $Q^{2}$ dependence (see Sections 7.1 and 7.4 , and Ref. [135]).

Similarly, the photo-absorption asymmetry $A_{2}$ can be expressed in terms of $A_{\perp}$ and $A_{1}$, where we use the global fit of $A_{1}$,

$$
A_{2}=\zeta A_{1}+\frac{A_{\perp}\left(x \cdot Q^{2}\right)}{d}
$$

Figure 6.1 shows the extracted $A_{2}$ as a function of $Q^{2}$ for selected bins in $x$ for both the proton and deuteron; $A_{2}$ does not exhibit any evidence for $Q^{2}$ dependence [42]. 
Table 6.3 contains the $A_{2}$ data for each spectrometer for both target materials.

\subsection{Spin Structure Functions. $g_{1} \& g_{2}$}

Instead of simply using $A_{\|}$and $A_{\perp}$ in Equation 1.53. $g_{1}$ may be determined from $A_{\|}$and $g_{2}$, where $g_{2}=g_{2}^{W W}$ may be assumed. [28]

$$
g_{1}=\frac{F_{1}}{D^{\prime}} A_{\| l} \frac{E+E^{\prime}}{E+E^{\prime} \cos \theta}+g_{2}^{w W} \frac{2 M x}{E+E^{\prime} \cos \theta} .
$$

Tables 6.1 and 6.2 contain the final E1.5.5 collaboration results for $g_{1}^{p}$ and $g_{1}^{d}$. The data have been rebinned from the original 38 bins over the $x$ range into 11 bins: this binning is consistent with that used by the SMC collaboration. and has thus been chosen to make comparisons between datasets. The data is shown in Fig. 6.3 after it has been evolved to $Q^{2}=5(\mathrm{GeV} / c)^{2}$, and combined between spectrometers. The process of evolving the data to the common $Q^{2}$ is discussed in Section 7.1 .

Writing $g_{2}$ in terms of $A_{\perp}$ and $A_{1}$,

$$
g_{2}=\frac{F_{2}}{2 x \gamma(1+R)}\left[\frac{A_{\perp}\left(x, Q^{2}\right)}{d}+A_{1}(\zeta-\gamma)\right] \text {. }
$$

where the contribution from $A_{1}$ is small [42]. The E1.5.5 data [42] on $g_{2}$ shows no indication of $Q^{2}$ dependence. so the spectrometer data shown in Table 6.3 is averaged for each $x$ bin to produce the data presented as $x g_{2}$ in Fig. 6.2.

\subsection{Extraction of Neutron Asymmetries and Structure Functions}

By measuring the deep inelastic scattering asymmetries of the proton and the deuteron, the DIS asymmetries of the neutron may be determined. The deuteron is a spin-1 nucleus, either in an S-state with the proton and neutron spins aligned with the deuteron spin, or in an D-state with the proton and neutron spins anti-aligned with the deuteron spin. The relationship between the deuteron structure functions and the proton and neutron structure functions is

$$
g_{(1,2)}^{d}=\frac{1-1.5 \omega_{d}}{2}\left[g_{(1,2)}^{p}+g_{(1,2)}^{n}\right]
$$




\begin{tabular}{ccccc}
\hline \hline & $\langle x\rangle$ & $\left\langle Q^{2}\right\rangle$ & $g_{1} / F_{1} \pm$ stat \pm syst & $g_{1} \pm$ stat \pm syst \\
\hline $2.75^{\circ}$ & 0.015 & 1.22 & $0.048 \pm 0.009 \pm 0.004$ & $0.358 \pm 0.069 \pm 0.035$ \\
& 0.025 & 1.59 & $0.057 \pm 0.008 \pm 0.006$ & $0.281 \pm 0.040 \pm 0.030$ \\
0.035 & 2.05 & $0.070 \pm 0.008 \pm 0.007$ & $0.266 \pm 0.030 \pm 0.027$ \\
0.050 & 2.58 & $0.111 \pm 0.009 \pm 0.009$ & $0.313 \pm 0.024 \pm 0.025$ \\
0.080 & 3.24 & $0.155 \pm 0.009 \pm 0.013$ & $0.287 \pm 0.017 \pm 0.023$ \\
& 0.125 & 4.03 & $0.186 \pm 0.012 \pm 0.018$ & $0.228 \pm 0.015 \pm 0.022$ \\
0.175 & 4.62 & $0.273 \pm 0.023 \pm 0.023$ & $0.240 \pm 0.020 \pm 0.020$ \\
0.250 & 5.06 & $0.358 \pm 0.023 \pm 0.030$ & $0.210 \pm 0.014 \pm 0.018$ \\
0.350 & 5.51 & $0.424 \pm 0.049 \pm 0.039$ & $0.150 \pm 0.017 \pm 0.014$ \\
0.500 & 5.77 & $0.561 \pm 0.058 \pm 0.048$ & $0.088 \pm 0.009 \pm 0.008$ \\
\hline $5.5^{\circ}$ & 0.050 & 4.01 & $0.222 \pm 0.088 \pm 0.009$ & $0.690 \pm 0.273 \pm 0.031$ \\
0.080 & 5.36 & $0.150 \pm 0.011 \pm 0.013$ & $0.303 \pm 0.022 \pm 0.026$ \\
0.125 & 7.17 & $0.209 \pm 0.007 \pm 0.018$ & $0.274 \pm 0.010 \pm 0.023$ \\
0.175 & 8.90 & $0.247 \pm 0.012 \pm 0.023$ & $0.226 \pm 0.011 \pm 0.021$ \\
0.250 & 10.64 & $0.353 \pm 0.011 \pm 0.030$ & $0.206 \pm 0.006 \pm 0.018$ \\
0.350 & 12.60 & $0.466 \pm 0.020 \pm 0.039$ & $0.154 \pm 0.007 \pm 0.013$ \\
0.500 & 14.02 & $0.561 \pm 0.024 \pm 0.048$ & $0.072 \pm 0.003 \pm 0.006$ \\
0.750 & 15.70 & $0.622 \pm 0.091 \pm 0.050$ & $0.009 \pm 0.001 \pm 0.001$ \\
\hline $10.5^{\circ}$ & 0.125 & 10.99 & $0.307 \pm 0.050 \pm 0.018$ & $0.415 \pm 0.068 \pm 0.026$ \\
0.175 & 13.19 & $0.304 \pm 0.022 \pm 0.023$ & $0.282 \pm 0.020 \pm 0.022$ \\
0.250 & 17.21 & $0.396 \pm 0.014 \pm 0.030$ & $0.229 \pm 0.008 \pm 0.018$ \\
0.350 & 22.73 & $0.500 \pm 0.025 \pm 0.038$ & $0.157 \pm 0.008 \pm 0.012$ \\
0.500 & 26.86 & $0.507 \pm 0.042 \pm 0.048$ & $0.058 \pm 0.005 \pm 0.006$ \\
0.750 & 34.72 & $0.559 \pm 0.405 \pm 0.050$ & $0.006 \pm 0.004 \pm 0.001$
\end{tabular}

Table 6.1: E155 Proton results for $g_{1} / F_{1}$ and $g_{1} \cdot\left\langle Q^{2}\right\rangle$ is in $(\mathrm{GeV} / \mathrm{c})^{2}$. 


\begin{tabular}{ccccc}
\hline \hline$\langle x\rangle$ & $\left\langle Q^{2}\right\rangle$ & $g_{1} / F_{1} \pm$ stat \pm syst & $g_{1} \pm$ stat \pm syst \\
\hline $2.75^{\circ}$ & 0.015 & 1.22 & $-0.033 \pm 0.016 \pm 0.001$ & $-0.234 \pm 0.115 \pm 0.011$ \\
0.025 & 1.59 & $0.011 \pm 0.014 \pm 0.001$ & $0.051 \pm 0.068 \pm 0.006$ \\
0.035 & 2.05 & $0.016 \pm 0.014 \pm 0.001$ & $0.059 \pm 0.052 \pm 0.005$ \\
0.050 & 2.57 & $0.030 \pm 0.015 \pm 0.002$ & $0.082 \pm 0.041 \pm 0.004$ \\
0.080 & 3.24 & $0.059 \pm 0.016 \pm 0.003$ & $0.103 \pm 0.028 \pm 0.005$ \\
0.125 & 4.03 & $0.079 \pm 0.021 \pm 0.005$ & $0.089 \pm 0.024 \pm 0.006$ \\
0.175 & 4.62 & $0.107 \pm 0.041 \pm 0.008$ & $0.083 \pm 0.032 \pm 0.006$ \\
0.250 & 5.06 & $0.192 \pm 0.042 \pm 0.012$ & $0.096 \pm 0.021 \pm 0.006$ \\
0.350 & 5.51 & $0.301 \pm 0.098 \pm 0.017$ & $0.086 \pm 0.028 \pm 0.005$ \\
0.500 & 5.77 & $0.389 \pm 0.129 \pm 0.024$ & $0.047 \pm 0.016 \pm 0.003$ \\
\hline $5.5^{\circ}$ & 0.050 & 4.00 & $-0.266 \pm 0.170 \pm 0.002$ & $-0.787 \pm 0.504 \pm 0.006$ \\
0.080 & 5.37 & $0.006 \pm 0.020 \pm 0.003$ & $0.012 \pm 0.038 \pm 0.006$ \\
0.125 & 7.16 & $0.079 \pm 0.014 \pm 0.005$ & $0.094 \pm 0.016 \pm 0.006$ \\
0.175 & 8.90 & $0.099 \pm 0.022 \pm 0.008$ & $0.080 \pm 0.018 \pm 0.006$ \\
0.250 & 10.62 & $0.184 \pm 0.020 \pm 0.012$ & $0.090 \pm 0.010 \pm 0.006$ \\
0.350 & 12.59 & $0.305 \pm 0.044 \pm 0.017$ & $0.081 \pm 0.012 \pm 0.005$ \\
0.500 & 14.01 & $0.349 \pm 0.057 \pm 0.025$ & $0.034 \pm 0.006 \pm 0.002$ \\
0.750 & 15.73 & $0.576 \pm 0.220 \pm 0.029$ & $0.006 \pm 0.002 \pm 0.001$ \\
\hline $10.5^{\circ}$ & 0.125 & 10.98 & $-0.020 \pm 0.095 \pm 0.006$ & $-0.025 \pm 0.117 \pm 0.008$ \\
0.175 & 13.19 & $0.157 \pm 0.042 \pm 0.008$ & $0.127 \pm 0.034 \pm 0.007$ \\
0.250 & 17.22 & $0.194 \pm 0.027 \pm 0.012$ & $0.094 \pm 0.013 \pm 0.006$ \\
0.350 & 22.65 & $0.309 \pm 0.053 \pm 0.017$ & $0.077 \pm 0.013 \pm 0.005$ \\
0.500 & 26.97 & $0.330 \pm 0.092 \pm 0.024$ & $0.028 \pm 0.008 \pm 0.002$ \\
0.750 & 34.79 & $0.428 \pm 0.903 \pm 0.028$ & $0.003 \pm 0.007 \pm 0.001$
\end{tabular}

Table 6.2: E155 deuteron results for $g_{1} / F_{1}$ and $g_{1} \cdot\left\langle Q^{2}\right\rangle$ is in $(\mathrm{GeV} / c)^{2}$. 


\begin{tabular}{|c|c|c|c|c|c|}
\hline$x$ & $\left\langle Q^{2}\right\rangle$ & $A_{2}^{p}$ & $I g_{2}^{p}$ & $A_{2}^{d}$ & $x z_{2}^{d}$ \\
\hline \multicolumn{6}{|c|}{$\theta \approx 2.75^{\circ}$} \\
\hline 0.022 & 1.15 & $0.149 \pm 0.111$ & $0.439 \pm 0.335$ & $-0.036 \pm 0.074$ & $-0.103 \pm 0.212$ \\
\hline 0.026 & 1.32 & $-0.020 \pm 0.032$ & $-0.069 \pm 0.088$ & $0.023 \pm 0.021$ & $0.060 \pm 0.056$ \\
\hline 0.039 & 1.56 & $-0.034 \pm 0.025$ & $-0.090 \pm 0.05 \tau$ & $0.023 \pm 0.017$ & $0.043 \pm 0.035$ \\
\hline 0.062 & 1.94 & $0.025 \pm 0.033$ & $0.024 \pm 0.054$ & $0.012 \pm 0.021$ & $0.013 \pm 0.034$ \\
\hline 0.099 & 2.34 & $0.016 \pm 0.046$ & $-0.020 \pm 0.054$ & $0.040 \pm 0.031$ & $0.041 \pm 0.03-4$ \\
\hline 0.159 & 2.71 & $0.033 \pm 0.069$ & $-0.021 \pm 0.056$ & $0.061 \pm 0.047$ & $0.024 \pm 0.035$ \\
\hline 0.255 & 3.01 & $0.075 \pm 0.107$ & $-0.008 \pm 0.056$ & $-0.108 \pm 0.076$ & $-0.077 \pm 0.035$ \\
\hline 0.411 & 3.25 & $-0.004 \pm 0.212$ & $-0.049 \pm 0.056$ & $0.273 \pm 0.166$ & $0.032 \pm 0.036$ \\
\hline 0.621 & 3.37 & $-0.842 \pm 0.594$ & $-0.123 \pm 0.054$ & $0.501 \div 0.503$ & $0.019 \pm 0.035$ \\
\hline 0.796 & 3.42 & $-0.294 \pm 1.734$ & $-0.019 \pm 0.042$ & $-2.329 \pm 1.460$ & $-0.043 \pm 0.024$ \\
\hline \multicolumn{6}{|c|}{$\theta \approx 5.5^{\circ}$} \\
\hline 0.072 & 3.68 & $0.185 \pm 0.177$ & $0.367 \pm 0.370$ & $-0.177 \pm 0.117$ & $-0.353 \pm 0.231$ \\
\hline 0.104 & 4.84 & $-0.041 \pm 0.03 i$ & $-0.102 \pm 0.064$ & $0.034 \pm 0.024$ & $0.050 \pm 0.040$ \\
\hline 0.161 & 6.26 & $0.019 \pm 0.032$ & $-0.022 \pm 0.042$ & $0.007 \pm 0.022$ & $-0.007 \pm 0.02 \pi$ \\
\hline 0.256 & 7.76 & $0.098 \pm 0.044$ & $0.030 \pm 0.039$ & $0.048 \pm 0.033$ & $0.018 \pm 0.025$ \\
\hline 0.417 & 9.20 & $-0.004 \pm 0.079$ & $-0.059 \pm 0.034$ & $-0.030 \pm 0.063$ & $-0.032 \pm 0.022$ \\
\hline 0.625 & 10.23 & $0.021 \pm 0.211$ & $-0.02 \tau \pm 0.026$ & $-0.011 \pm 0.186$ & $-0.015 \pm 0.017$ \\
\hline 0.828 & 10.76 & $-0.298 \pm 0.701$ & $-0.010 \pm 0.014$ & $1.102 \pm 0.603$ & $0.012 \pm 0.008$ \\
\hline \multicolumn{6}{|c|}{$\theta \approx 10.5^{\circ}$} \\
\hline 0.168 & 9.77 & $0.034 \pm 0.071$ & $0.011 \pm 0.115$ & $-0.069 \pm 0.049$ & $-0.118 \pm 0.070$ \\
\hline 0.258 & 13.70 & $0.049 \pm 0.061$ & $-0.001 \pm 0.070$ & $0.006 \pm 0.044$ & $-0.016 \pm 0.043$ \\
\hline 0.432 & 20.06 & $0.100 \pm 0.082$ & $0.006 \pm 0.045$ & $0.016 \pm 0.068$ & $-0.013 \pm 0.029$ \\
\hline 0.643 & 25.46 & $0.186 \pm 0.136$ & $0.005 \pm 0.020$ & $0.357 \pm 0.133$ & $0.030 \pm 0.015$ \\
\hline 0.841 & 29.25 & $0.670 \pm 0.309$ & $0.008 \pm 0.006$ & $-0.295 \pm 0.322$ & $-0.006 \pm 0.004$ \\
\hline
\end{tabular}

Table 6.3: E155 $A_{2}$ and $x g_{2}$ for the proton and deuteron.

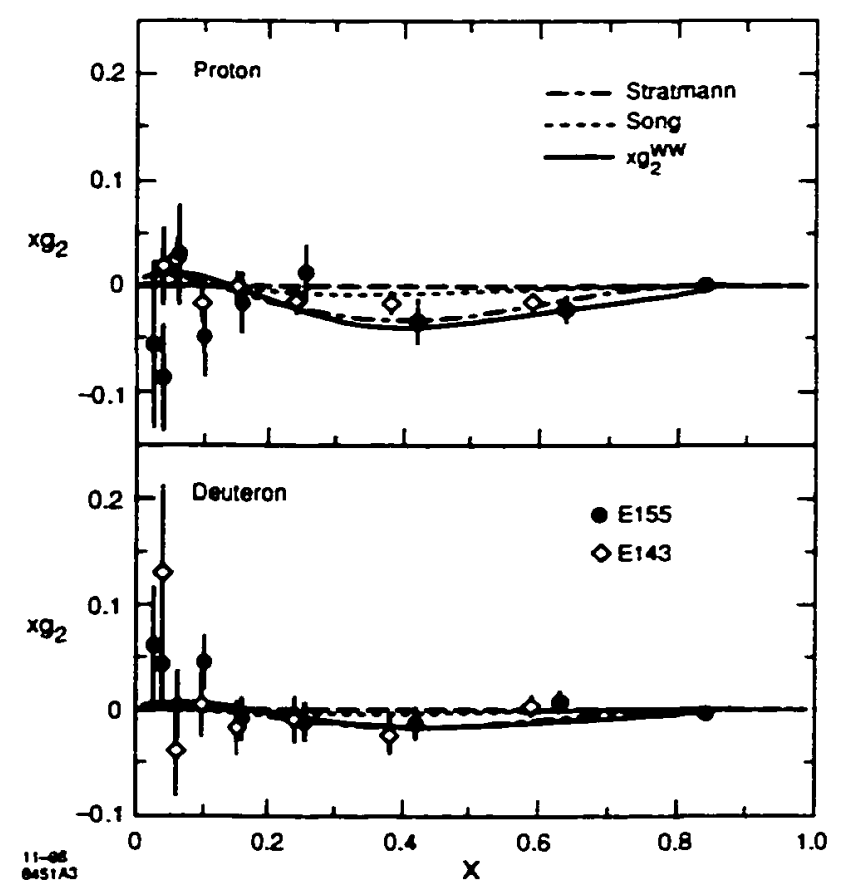

Figure 6.2: E155 $g_{2}$ results averaged over spectrometers. and data from E143 [28]. Also shown are the $g_{2}^{W W}$ at the average $Q^{2}$ value for each $x$ bin, and bag model calculations by Stratmann [13i] and Song [138]. Figure from [42]. 
where $\omega_{d}=0.05 \pm 0.01$ is the deuteron D-state probability.

Figure 6.3 shows the final E1.5.5 collaboration results for the structure function $g_{1}$ for the proton. deuteron. and extracted neutron. The spectrometer data have been evolved to a $Q_{0}^{2}=5(\mathrm{GeV} / \mathrm{c})^{2}$ and averaged in each $x$ bin. The process of evolving the data to the common $Q^{2}$ is discussed in Section 7.1 . 


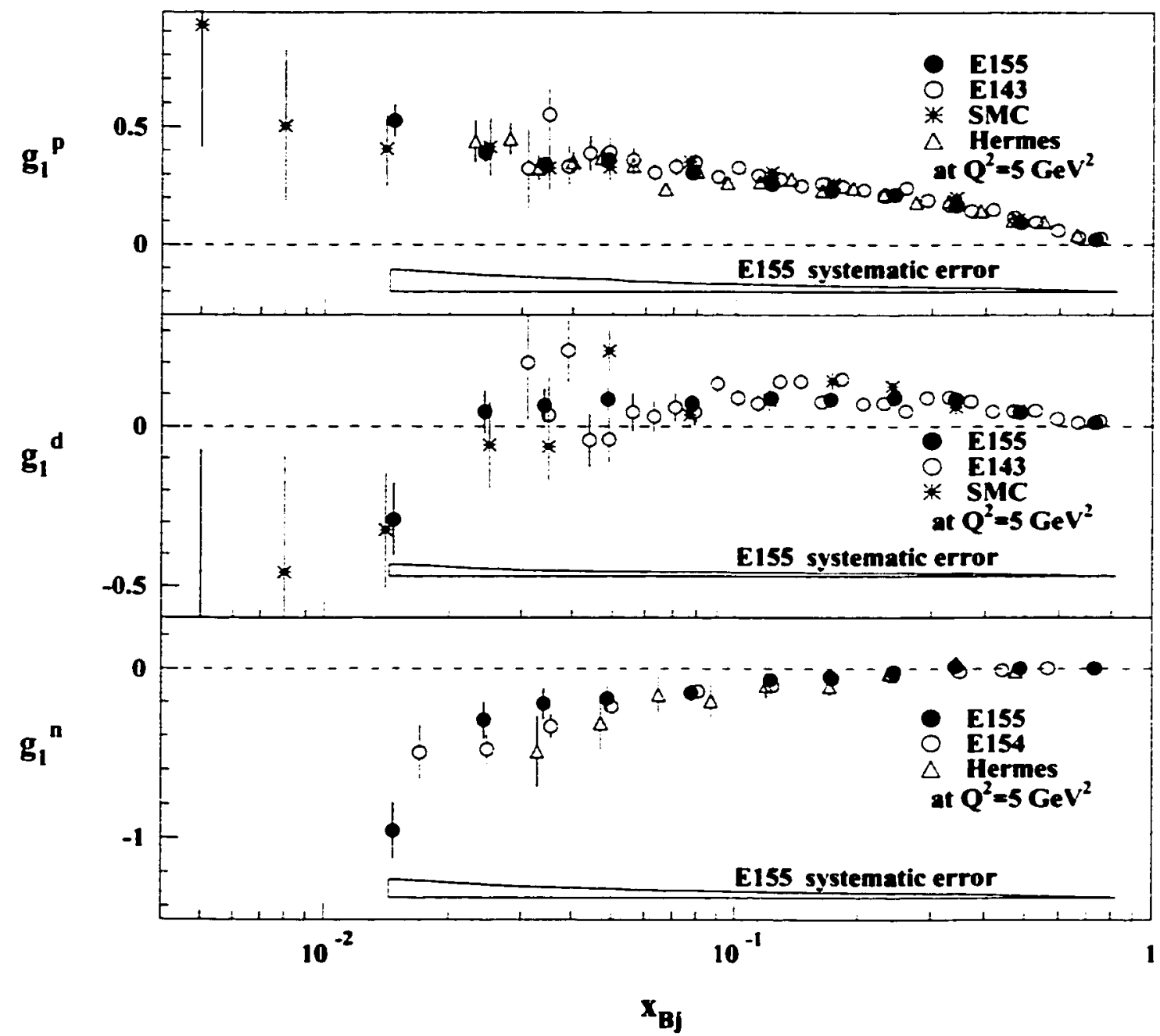

Figure 6.3: Combined $g_{1}$ from all three spectrometers evolved to a $Q^{2}$ of $5(\mathrm{GeV} / c)^{2}$. for the proton. deuteron, and extracted neutron. 


\section{CHAPTER 7 \\ INTERPRETATIONS}

The structure functions for the proton and deuteron can be interpreted using sum rules. comparisons to models. and analysis of the $Q^{2}$ dependencies either using a phenomenological fit or NLO fitting in QCD.

\section{$\underline{7.1 \quad \text { Evolving } g_{1} \text { to a common } Q^{2}}$}

For the evaluation of the integrals of the structure functions and the sum rules. the data were evolved to a common $Q_{0}^{2}=5(\mathrm{GeV} / \mathrm{c})^{2}$ using,

$$
\left(\frac{g_{1}\left(x, Q_{0}^{2}\right)}{F_{1}\left(x \cdot Q_{0}^{2}\right)}\right)=\left(\frac{g_{1}\left(x, Q^{2}\right)}{F_{1}\left(x \cdot Q^{2}\right)}\right)+\left[\left(\frac{g_{1}\left(x, Q_{0}^{2}\right)}{F_{1}\left(x \cdot Q_{0}^{2}\right)}\right)_{\text {fit }}-\left(\frac{g_{1}\left(x \cdot Q^{2}\right)}{F_{1}\left(x \cdot Q^{2}\right)}\right)_{\text {fit }}\right] \text {. }
$$

where the model of $g_{1} / F_{1}$ comes from a global fit to the world data [139] (similar

to the $A_{1}$ global fit [134]) and is evaluated at the measured $Q^{2}$ and at the common $Q_{0}^{2}=5(\mathrm{GeV} / c)^{2}$. This technique was used instead of simply taking

$$
\left(\frac{g_{1}\left(x, Q_{0}^{2}\right)}{F_{1}\left(x \cdot Q_{0}^{2}\right)}\right)=\left(\frac{g_{1}\left(x \cdot Q^{2}\right)}{F_{1}\left(x \cdot Q^{2}\right)}\right)
$$

because the use of Eq. 7.1 includes the observed $Q^{2}$ dependence of $g_{1} / F_{1}$.

Figures 7.1 and 7.2 show the residual $Q^{2}$ dependence of the ratio $g_{1} / F_{1}$ : also shown are data from previous SLAC (E143). DESY (HERMES), and CERN (EIIC. SMC) measurements. The data from all experiments have been rebinned into the twelve bins in $x$ shown in Fig. 7.1 and 7.2 with small bin centering corrections calculated using the global fit to $g_{1} / F_{1}$ [136]. The data from each spectrometer has been averaged together to create a single datum in each $x$ bin: in bins with three E15.5 data points, they correspond to the three spectrometers. 


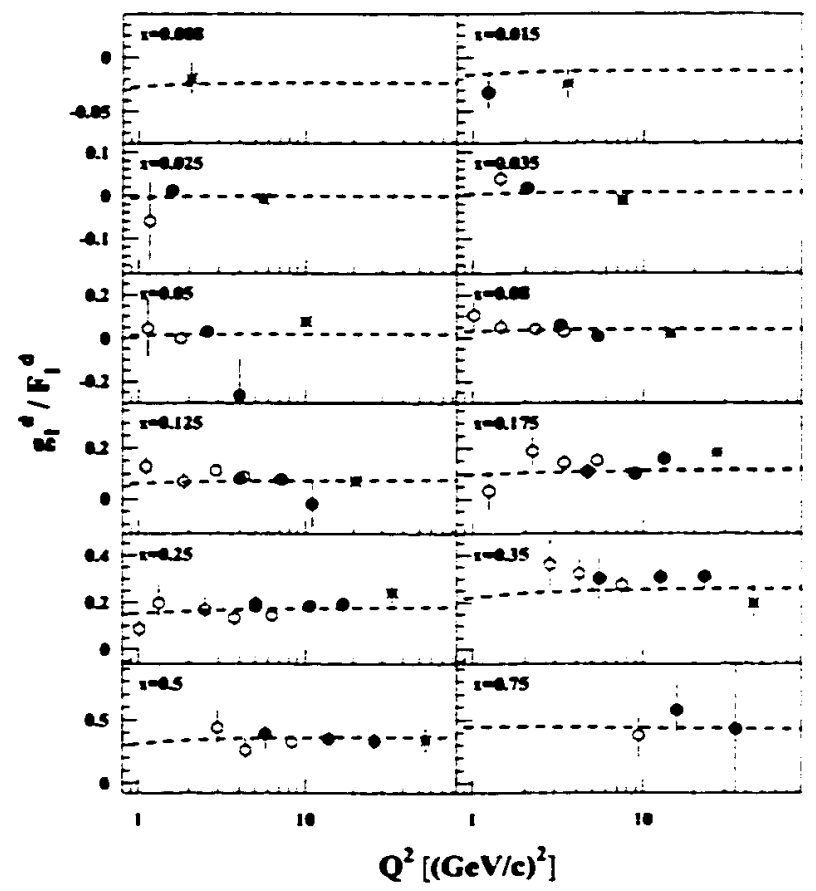

Figure $7.1: g_{1} / F_{1}$ for the deuteron. The dashed line is the global fit to $g_{1} / F_{1}$. Error bars are statistical only. The data shown (other than E 1.55) come from the following references: E143. Ref. [28]: SMC. Ref. [140].

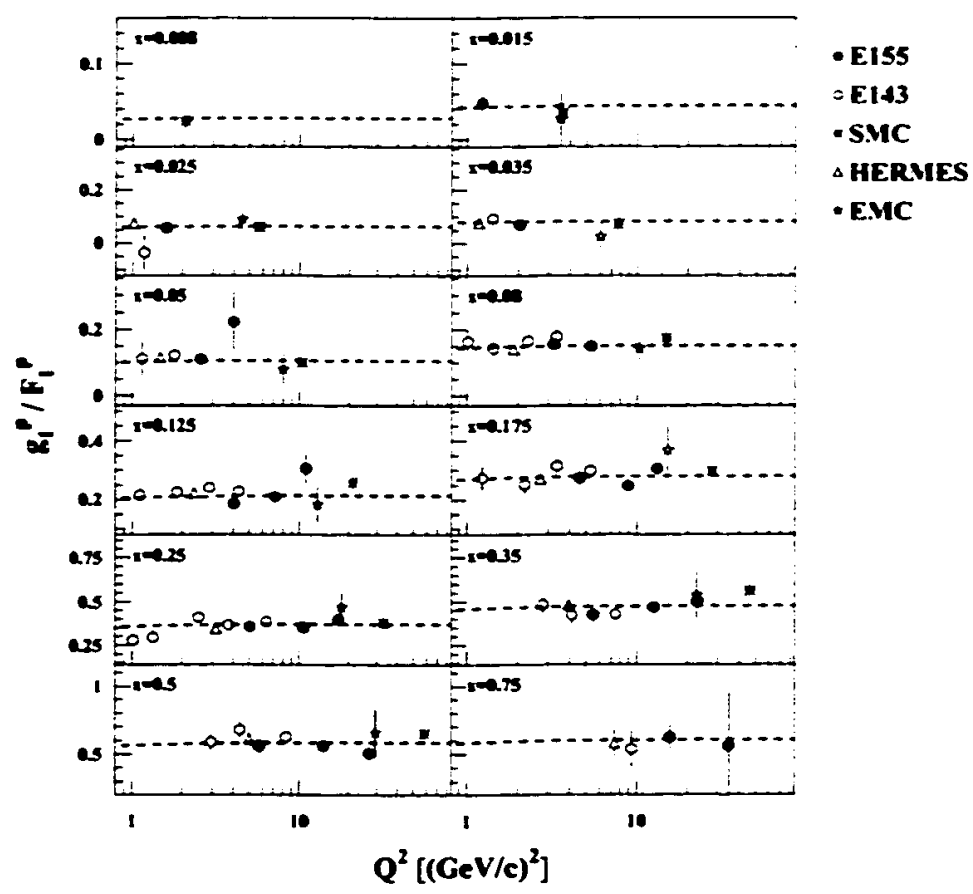

Figure $7.2: g_{1} / F_{1}$ for the proton. The dashed line is the global fit to $g_{1} / F_{1}$. Error bars are statistical only. The data shown (other than E155) come from the following references: E143, Ref. [28]: SMC, Ref. [140]; EMC, Ref. [35]; HERMES, Ref. [39]. 


\subsection{Sum rules involving $g_{2}$}

The Burkhardt-Cottingham sum rule for $g_{2}$ is. for large $Q^{2}$,

$$
\int_{0}^{1} g_{2}(x) d x=0
$$

The E155 data was used to evaluate it in the measured region of $0.02 \leq x \leq 0.8$ at $Q_{0}^{2}=5(\mathrm{GeV} / \mathrm{c})^{2}$. by assuming that all of the $Q^{2}$ dependence in $g_{2}$ is in $g_{2}^{W W}[42]$. The proton measured region integral was $-0.022 \pm 0.071$ and the deuteron result was $0.023 \pm 0.044$. both of which are consistent with the predicted value of zero [42]. However, since the $x \rightarrow 0$ behavior of $g_{2}$ is not known. a large low $x$ contribution could violate the sum rule.

The Efremov-Leader-Teryaev (ELT) sum rule involves the valence quark contributions to $g_{1}$ and $g_{2}$,

$$
\int_{0}^{1} x\left(g_{1}^{v}(x)+2 g_{2}^{v}(x)\right) d x=0
$$

If the sea quarks are the same in the proton and the neutron. then the ELT sum rule takes the form.

$$
\int_{0}^{1} x\left(g_{1}^{p}(x)+2 g_{2}^{p}(x)-g_{1}^{n}(x)-2 g_{2}^{n}(x)\right) d x=0 .
$$

Using the E1.5.5 data in the measured region evolved to $Q_{0}^{2}=5(\mathrm{GeV} / c)^{2}$. gives an ELT sum rule result of $-0.015 \pm 0.036$, consistent with zero[42]. The contribution from the low $x$ extrapolation is, in this case, suppressed by the factor of $x$.

\subsection{The Operator Product Expansion Matrix Element. $d_{2}$}

The twist-3 matrix elements $d_{n}$ from the operator product expansion (OPE) are related to the moments of $\overline{g_{2}}=g_{2}-g_{2}^{W W}$,

$$
d_{n}=\frac{2(n+1)}{n} \int_{0}^{1} x^{n} \overline{g_{2}}\left(x, Q^{2}\right) d x, n=2,4, \ldots
$$




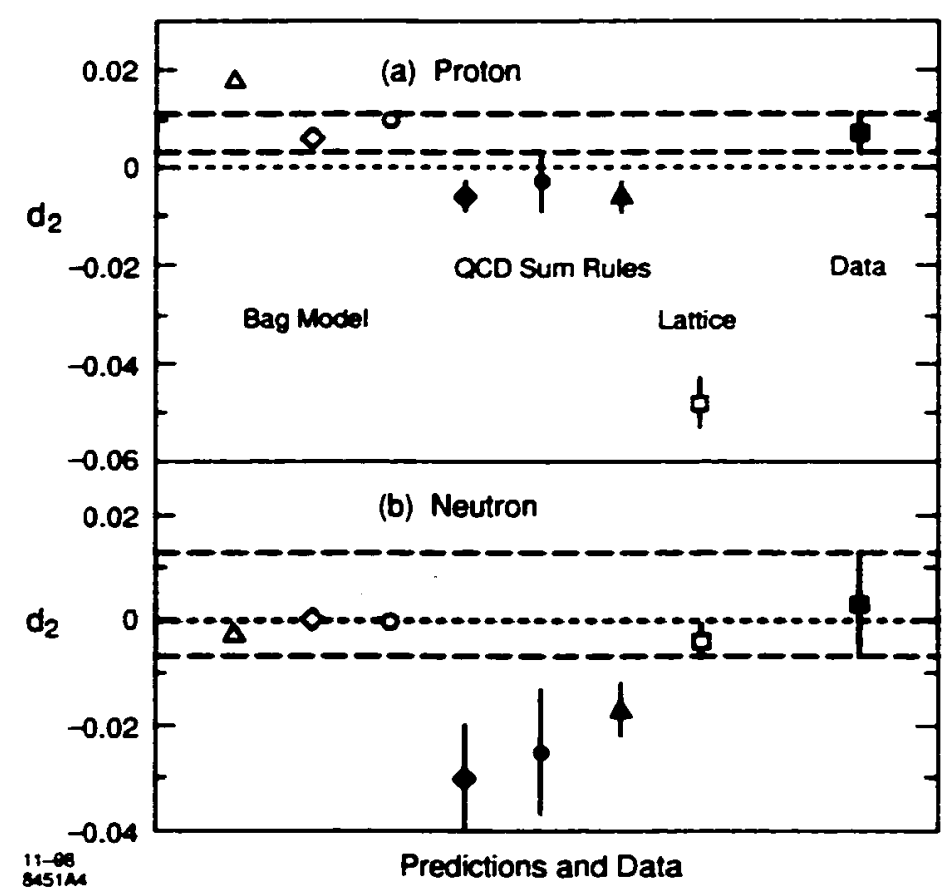

Figure 7.3: The combined SLAC determination of the leading twist-3 matrix element $d_{2}$ with model predictions: Bag models [138, 13T, 141]: QCD sum rules [142, 143. 144]: Lattice QCD [145]: and Chiral Soliton Model [146]. The lattice QCD prediction presented in Ref. [145], and displayed here, was flawed; the calculation has been revisted. and the new results are discussed in the text.

where $\left(1 / \sqrt{Q^{2}}\right)$ terms are neglected. For the E1.5.5 analysis. the $\overline{g_{2}}$ is interpreted as due to twist-3 quark-gluon correlations [42]. The matrix elements are sensitive to deviations from the leading twist- $2\left(g_{2}^{W W}\right)$ behavior of $g_{2}$. The E1.5.5 results for $d_{2}$. determined by assuming that $\overline{g_{2}}$ is independent of $Q^{2}$ in the measured region. are $d_{2}^{p}=0.005 \pm 0.008$ and $d_{2}^{d}=0.008 \pm 0.005$ [42]. Combining the E1.5.5 $g_{2}$ data with that from E142 [31], E143 [28], and E154 [33], yields the matrix elements for the proton and neutron of $d_{2}^{p}=0.007 \pm 0.004$ and $d_{2}^{n}=0.004 \pm 0.010$ [42]. These combined SLAC determinations of $d_{2}$ are shown in Fig. 7.3 compared to theoretical calculations using Bag Models, QCD sum rules. Chiral Soliton Model. and Lattice QCD. The predictions are comparable with the data (except for the proton lattice QCD calculation) within the errors. The lattice QCD calculations shown in Fig. 7.3 were flawed in that the twist-3 operator mixes non-perturbatively with lower- 
dimensioned operators under renormalization; the calculation of Ref. [14.5] did the renormalization in perturbation theory. The calculation has been revisited. where now the renormalization is performed non-perturbatively [14i]: the $n=2$ moments of $g_{2}^{p \cdot n}$ from Ref. [147] are

$$
\begin{aligned}
& \left.\Gamma_{2}^{(2)}(p)=\int_{0}^{1} x^{2} g_{2}^{p}(x) d x=0.00\right\rceil \pm 0.015 \\
& \left.\Gamma_{2}^{(2)}(n)=\int_{0}^{1} x^{2} g_{2}^{n}(x) d x=0.006 \pm 0.00\right\rceil
\end{aligned}
$$

Esing these values and the values of $a_{2}$ from Ref. [14.5]. the reduced matrix elements are

$$
\begin{aligned}
& d_{2}^{p}=0.051 \pm 0.039 \\
& d_{2}^{n}=0.01 \tau \pm 0.01 \tau
\end{aligned}
$$

where the error on $d_{2}$ has been calculated using $\sigma\left(d_{2}\right)=\sigma\left(\Gamma_{2}^{(2)}\right)-\sigma\left(a_{2}\right)$. which seems to be consistent with the treatment of errors in Ref. [145].

\section{$7.4 Q^{2}$ Dependence and The Role of the $10.5^{\circ}$ Spectrometer}

The E1.5.5 data cover the $Q^{2}$ range between the E143/HERMES data and the EMC/SMC data. E15.5 can be used by itself to investigate the $Q^{2}$ dependence of $g_{1}$ (or $g_{1} / F_{1}$ or $A_{1}$ ), but in concert with the other world data, the E1.5.5 data make a important contribution.

The high $Q^{2}$ points in E1.55 are provided by the $10.5^{\circ}$ spectrometer. It can be seen in Figures 7.1 and 7.2 that the $10.5^{\circ}$ data are at about the same kinematics as the EMC data, with smaller errors.

Global fits to the world data set were done for both $g_{1} / F_{1}$ and $f_{1}$ to facilitate the calculation of radiative corrections [135]. By investigating the change in the fit parameters and errors as the E15.5 data is included in the fits. the impact of the E15.5 data can be investigated. 


\begin{tabular}{|c|c|c|c|}
\hline Parameter & $\begin{array}{l}\text { World Data } \\
\text { Without E15.5 }\end{array}$ & $\begin{array}{c}\text { With E15.5 } \\
2.75^{\circ} \& 5.5^{\circ} \text { Data }\end{array}$ & $\begin{array}{l}\text { With All } \\
\text { E15.5 Data }\end{array}$ \\
\hline$a$ & $0.8114 \pm 0.1497$ & $0.8154 \pm 0.1038$ & $0.7971 \pm 0.0838$ \\
\hline$b$ & $0.9985 \pm 0.4313$ & $0.8885 \pm 0.2780$ & $1.0585 \pm 0.2017$ \\
\hline$c$ & $-1.1052 \pm 0.5157$ & $-0.889 .5 \pm 0.326 .5$ & $-1.15 .33 \pm 0.2771$ \\
\hline$d$ & $-0.1479 \pm 0.0365$ & $-0.1461 \pm 0.03330$ & $-0.1588 \pm 0.0324$ \\
\hline$\alpha$ & $0.6618 \pm 0.0542$ & $0.6589 \pm 0.0400$ & $0.6 .5 .30 \pm 0.03 .51$ \\
\hline$a^{\prime}$ & $-0.1898 \pm 0.1078$ & $-0.1699 \pm 0.1014$ & $-0.1683 \pm 0.1025$ \\
\hline$b^{\prime}$ & $0.2225 \pm 0.6248$ & $0.0684 \pm 0.54 .59$ & $0.0547 \pm 0.5504$ \\
\hline$c^{\prime}$ & $0.1947 \pm 1.1236$ & $0.5763 \pm 0.9273$ & $0.6021 \pm 0.9348$ \\
\hline$d^{\prime}$ & $-0.0600 \pm 0.1938$ & $-0.0573 \pm 0.1967$ & $-0.0699 \pm 0.1927$ \\
\hline 3 & $0.2238 \pm 0.1530$ & $0.2048 \pm 0.1601$ & $0.2006 \pm 0.1624$ \\
\hline
\end{tabular}

Table 7.1: The parameters of the fit in Equations $7.11-\bar{\tau} .13$ with their errors for three sets of world data: excluding E155, including the E15.5 $2.75^{\circ}$ and $5.5^{\circ}$ spectrometers, and including all of the E15.5 data.

Table 7.1 shows the parameters for the global fit to $A_{1}[1: 35]$ as given by

$$
\begin{aligned}
& A_{1}^{p}\left(x, Q^{2}\right)=x^{\alpha}\left(a+b x+c x^{2}\right)\left(1+d / Q^{2}\right) \\
& A_{1}^{n}\left(x, Q^{2}\right)=x^{\beta}\left(a^{\prime}+b^{\prime} x+c^{\prime} x^{2}\right)\left(1+d^{\prime} / Q^{2}\right) \\
& A_{1}^{d}\left(x, Q^{2}\right)=\left(1-1.5 \omega_{D}\right) \frac{A_{1}^{p}\left(x, Q^{2}\right) F_{1}^{p}\left(x \cdot Q^{2}\right)+A_{1}^{n}\left(x, Q^{2}\right) F_{1}^{n}\left(x, Q^{2}\right)}{2 F_{1}^{d}\left(x \cdot Q^{2}\right)}
\end{aligned}
$$

The fitting was done using MIGRAD: after the parameter values had converged. the errors fell into a repeating pattern as the fit was further iterated (error values were identical for every third iteration). The errors seemed to oscillate from a minimization of the proton coefficients through to a minimization of the neutron coefficients, with an intermediate state. The parameter errors listed in Table 7.1 are the set of errors for which the proton errors were minimized. Notice that the inclusion of the $10.5^{\circ}$ data reduces the errors on the fit parameters significantly.

\subsection{NLO Fitting}

As discussed in Section 1.4, fitting the data using next-to-leading order (NLO) perturbative quantum chromodynamics (pQCD) interprets the structure functions in terms of polarized parton distributions which evolve in $Q^{2}$ according to the 
DGLAP $[19,20,21,22]$ equations. Once fitting has been accomplished. the polarized parton distributions may be used to extract $\Delta \Sigma . \Delta G, \Gamma_{1}^{p}, \Gamma_{1}^{n}$, and $\Gamma_{1}^{p-n}$. The E15.5 NLO analysis follows the same prescription as the E154 NLO analysis [41]. The results discussed below are from G. Mitchell's "a8" analysis of July 26, 2000.

The polarized parton distributions are parameterized in terms of the GRV98 unpolarized parton distributions (listed in Table 1.1) at the input scale. $\mu^{2}=$ $0.40(\mathrm{GeV} / \mathrm{c})^{2}$. as

$$
\Delta q_{i}\left(x, \mu^{2}\right)=A_{i} x^{\alpha_{i}}(1-x)^{\beta_{i}} q_{i}\left(x, \mu^{2}\right)
$$

where $\Delta q_{i}$ are the polarized parton distributions. and $q_{i}\left(x, \mu^{2}\right)$ are the unpolarized parton distributions for the quarks. anti-quarks. and gluons. The $3_{i}$ coefficients were held fixed at zero for the E15.5 NLO fit, as they were for the E1.54 analysis [41]. The polarized sea is assumed to be isospin-symmetric $(\Delta \bar{u}=\Delta \bar{d})$. resulting in the sea quark contribution to $g_{1}$ being parameterized by $\Delta \bar{Q}=\frac{1}{2}(\Delta \bar{u}+\Delta \bar{d})+\frac{1}{5} \Delta \bar{s}$ [41]. Therefore. four parton distributions are used: valence quarks (non-singlet). $u_{v}=u-\bar{u}$ and $d_{v}=d-\bar{d}$; sea quarks $\bar{Q}$ (singlet): and gluons. $C_{r}$ (singlet). In this evaluation the GRV98 solution for $\alpha_{s}\left(Q^{2}\right)$ [24] was used. in which $\alpha_{s}\left(M_{Z}^{2}\right)=0.114$ and $\alpha_{s}\left(5(\mathrm{GeV} / c)^{2}\right)=0.26[148]$.

The polarized parton distributions are evolved from $Q^{2}=\mu^{2}$ to the experimental $Q^{2}$ using the NLO DGLAP equations. in the $\overline{\mathrm{MS}}$ scheme [148]. The evolved distributions are used to calculate $g_{1}$ using.

$$
g_{1}\left(x, Q^{2}\right)=\frac{1}{2} \sum_{i}^{N_{f}} e_{i}^{2}\left[C_{q} \odot \Delta q_{i}+\frac{1}{N_{f}} C_{G} \odot \Delta G\right] .
$$

where $N_{f}$ is the number of active quark flavors [41]. The Wilson coefficients. $C_{7}$ and $C_{G}$, are perturbative series in $\alpha_{S}$, calculated in the $\overline{\mathrm{MS}}$ scheme: the leading order terms are $C_{q}(x)=\delta(1-x)$ and $C_{G}(x)=0$, which reproduce the naive QP.M result of $g_{1}=\frac{1}{2} \sum_{i} e_{i}^{2} \Delta q_{i}[41]$. This yields $g_{1}\left(x, Q^{2}\right)$ parameterized by $A_{i}$ and $\alpha_{i}$ for each of the four distributions $\Delta u_{v}, \Delta d_{v}, \Delta \bar{Q}$ and $\Delta G$. Table 7.2 contains the coefficients 


\begin{tabular}{|c|c|c|c|}
\hline & Value & Stat. & Syst. \\
\hline$A_{u}$ & 0.95 & $\begin{array}{l}+0.04 \\
-0.01\end{array}$ & $\begin{array}{l}+0.05 \\
-0.04\end{array}$ \\
\hline$A_{d}$ & -0.42 & $\begin{array}{l}+0.02 \\
-0.04\end{array}$ & $\begin{array}{l}+0.02 \\
-0.04\end{array}$ \\
\hline$A_{Q}$ & 0.01 & $\begin{array}{l}+0.47 \\
-0.01\end{array}$ & $\begin{array}{l}+0.50 \\
+0.12\end{array}$ \\
\hline$A_{g}$ & 0.50 & $\begin{array}{l}+0.37 \\
-0.54\end{array}$ & $\begin{array}{l}+0.48 \\
-1.13\end{array}$ \\
\hline$\alpha_{u}$ & 0.57 & $\begin{array}{l}+0.05 \\
-0.01\end{array}$ & $\begin{array}{l}+0.04 \\
-0.02\end{array}$ \\
\hline$\alpha_{d}$ & 0.00 & +0.00 & +0.01 \\
\hline$\alpha_{Q}$ & 1.00 & $\begin{array}{l}+0.24 \\
-0.24\end{array}$ & $\begin{array}{r}+0.43 \\
-0.29\end{array}$ \\
\hline$\alpha_{g}$ & 0.02 & $\begin{array}{l}+0.66 \\
-0.02\end{array}$ & $\begin{array}{l}+0.78 \\
-0.02\end{array}$ \\
\hline
\end{tabular}

Table 7.2 : Coefficients of the NLO PQCD fit of the polarized parton distributions shown in Eq. 7.14 .

of the E15.5 NLO fit.

Using the polarized parton distributions to evaluate the proton and deuteron integrals at a $Q^{2}=5(\mathrm{GeV} / \mathrm{c})^{2}$ yields

$$
\begin{aligned}
& \Gamma_{1}^{p}=0.118 \pm 0.004 \pm 0.00 \tau \\
& \Gamma_{1}^{n}=-0.058 \pm 0.005 \pm 0.008 \\
& \Gamma_{1}^{d}=0.028 \pm 0.004 \pm 0.005
\end{aligned}
$$

and the Bjorken sum rule result of

$$
\Gamma_{1}^{p-n}=0.176 \pm 0.003 \pm 0.00 \tau
$$

Which is consistent with the prediction for the Bjorken sum rule with QCD corrections to order $\alpha_{s}^{3}$ evaluated at $Q^{2}=5(\mathrm{GeV} / \mathrm{c})^{2}$ of $0.182 \pm 0.005$.

Integrating the polarized parton distributions to determine the quark and gluon helicity contributions to the nucleon yields,

$$
\begin{aligned}
& \Delta \Sigma=0.229 \pm 0.041 \pm 0.057 \\
& \Delta G=1.62 \pm 0.78 \pm 1.13
\end{aligned}
$$

The quark helicity contribution to the nucleon is $\frac{1}{2} \Delta \Sigma$. so the quarks contribute about one quarter of the total nucleon spin. The gluon spin contribution. $\triangle G$ 
is poorly constrained by this measurement; still the indication is that the gluons contribute significantly to the nucleon spin, and contribution (or cancellation) from orbital angular momentum can not be ruled out.

\section{$\underline{7.6}$ Conclusion}

E15.5 has precise data for $g_{1}^{p}\left(x, Q^{2}\right)$ and $g_{1}^{d}\left(x, Q^{2}\right)$ over a wide range in $x$ and $Q^{2}$. The data are consistent with the other world measurements. and the combined data achieve sufficient coverage and precision to allow interpretation of the structure functions in terms of the polarized parton distributions using NLO pQCD fitting.

The E155 data on $g_{2}^{p}\left(x, Q^{2}\right)$ and $g_{2}^{d}\left(x, Q^{2}\right)$ are not of high precision. and cannot distinguish between the model predictions. The extension run. E15.5X. collected precise data on $g_{2}^{p}\left(x, Q^{2}\right)$ and $g_{2}^{d}\left(x, Q^{2}\right)$, and will improve the errors on the world data set by about a factor of three.

The current world data on $g_{1}$ are able to constrain $\Delta \Sigma$ well. indicating a small quark contribution to the nucleon spin. The data are not able to strongly constrain the polarized gluon distribution, but do suggest that the gluons provide a significant contribution. Further advances in understanding the nucleon spin would seem to require probes directly sensitive to the gluon distribution, such as open charm photoproduction or production in electron or muon scattering. The HERMES experiment at DESY measures the structure functions in semi-inclusive DIS. providing additional understanding of the nucleon spin structure. 


\section{APPENDIX A}

\section{E155X: Measuring $A_{\perp}$}

For the extension run. E15.5X, several modifications were made to the $10.5^{\circ}$ spectrometer. A thin passive preradiator was installed in front of the shower calorimeter. to increase the signal in the active preradiator bars. New hodoscopes were installed in both the front and rear of the spectrometer to improve the tracking resolution.

\section{A.1 Lead Passive Preradiator (PbPPR)}

Calculations in GEANT performed by Todd Averett indicated that a passive radiator of one radiation length located in front of the preradiator would increase the electron signal in the preradiator, and would not degrade the energy resolution in the shower calorimeter by too much. During part of the T418 test run. a sheet of lead $\frac{1}{4}^{-}$thick was propped in front of the PR to test the PR response.

For E15.5X. the lead passive preradiator was installed to cover the entire face of the preradiator. The lead passive preradiator was a pair of $\frac{1}{8}^{*}$ thick sheets of lead sandwiched together, measuring about $i T \mathrm{~cm}$ wide and $i 0 \mathrm{~cm}$ tall. This provided a 1.1 radiation length passive radiator in front of the preradiator. The effect of having the passive preradiator in place was to increase the signal in the preradiator. such that it was possible to determine an energy signal for the preradiator.

\section{A.2 Rear $10.5^{\circ}$ Hodoscope Design}

To achieve the desired vertical position resolution at the downstream end, a rear hodoscope was designed, consisting of two planes of 1 " wide fingers, offset from each 
other by $0.5 "$. To cover the acceptance, the forward plane would have 24 fingers. and the rear plane 25 fingers. This gives an active area approximately $25 \%(6: 3.5 \mathrm{~cm})$ tall. which is a good match to the $62.4 \mathrm{~cm}$ height of the preradiator stack. which defines the acceptance for the $10.5^{\circ}$ spectrometer.

\section{A.2.1 Construction}

A primary design goal for the rear hodoscope package was to be able to install the package between the Cherenkov tank and the shower calorimeter without moving either of the existing detectors. In order to fit on the shower calorimeter frame between the preradiator stack and the Cherenkov tank. the hodoscope frame could then extend only $5.5^{\cdots}$ in front of the PR. and could have a maximum height of 29.375”. Further limiting the size of the hodoscope. the PR forward support bars extend 1.5 " in front of the PR stack, and are located at $X$ positions of approximately $\pm 1.5 .75 "$ from the shower calorimeter center line. Therefore to install the rear hodoscope, without moving the shower calorimeter or Cherenkov tank. would require a hodoscope package no thicker than $4^{*}$. and no taller than $29.37 .5^{-}$. Due to the tilt of the Cherenkov tank. if the package height is shorter than the maximum. the thickness may be somewhat larger than 4 ".

The desired size of the hodoscope acceptance is based on the acceptance of the preradiator stack. The height of the preradiator stack is $62.4 \mathrm{~cm}$, and the width of the preradiator stack is $75.0 \mathrm{~cm}$. Since the minimum height of the hodoscope's active area is $24.6 "$, the upstream and downstream planes have 24 and 2.51 " wide fingers respectively. The hodoscope was designed to have a $29.5 "(74.9 \mathrm{~cm})$ wide open acceptance. The scintillator fingers were cut $30 \%$ long. so a small amount of material extends into the support framing. 
Finger Assembly

Each scintillator finger is $30^{-}$long, $0.375^{*}$ thick, and $1.0^{-}$wide. Most of the cutting of the fingers was done by the UVa hodoscope group. The PMTs available for this project are 0.75 diameter. Unfortunately. we do not seem to have complete documentation for these PMTs, so we are not sure of their proper specifications. A few tubes look like XP1110 tubes (manufactured by RCA), but most of the tubes seem to be a somewhat different type. In the absence of their specifications. we assume the XP1110 specifications hold.

Each scintillator finger is coupled to the PMT across a 1.5- long acrylic waveguide, consisting of a trapezoidal section of $0.375^{\circ}$ thick acrylic sheet which matches the $1^{*}$ width of the finger to the $0.75^{*}$ diameter of the tube, and a "button" cut from $0.75 \%$ acrylic rod with a $0.375 \%$ notch across the diameter to hold the trapezoid securely. Bicron BC-600 Optical Cement is used to couple the trapezoid to the button, and is also used to couple the waveguide to both the scintillator and the PMT.

There are two types of hodoscope fingers in the rear hodoscope. differentiated by the type of PMT base. There are 29 -short base" fingers which have a rectilinear base assembly attached directly to the tube. Most of the tubes are epoxied into their base socket. The 20 "G10 card" fingers have the tube epoxied into a socket from which wires extend to a G10 circuit card which plugs into the base assembly.

The "G10 card" fingers have a solid $\mu$-metal shield. For this installation. the $\mu$-metal shields had to be slightly shortened from their previous application. The $\mu$-metal shields were cut to a length of $4.25 \%$. The $\mu$-metal shields extend beyond the PMT face about halfway along the light guide $\left(\sim 0.755^{-*}-1^{-}\right.$beyond the face).

The "short base" tubes have $\mu$-metal shields made from a single layer of sheet $\mu$-metal. The $\mu$-metal shields cover the tubes from about half-way along the light- 
guides to the PMT base.

\section{$\underline{\text { Frame Design and Construction }}$}

The hodoscope frame as constructed is $28.25 \%$ in total height. $3.875^{-}$thick (neglecting the extension of the cover-plate bolts). and $52 \%$ wide. The frame has a 32" wide cutout in the rear facing, which is designed to fit around the PR angle aluminum brackets. The cutout is $1.0625^{\circ}$ deep. The lower frame plate is $1^{-}$thick aluminum, and the upper frame plate is $0.5 \%$ thick. The main vertical supports are the two side ladders, made from 0.5 square aluminum bar stock. The ladders have four rungs at very approximately $5 "$ spacing. The spacing is adjusted so that the rungs do not obstruct the the high voltage and signal cables.

The rear plane is comprised of 25 of the short-base fingers. The tubes and bases extend to the low theta side of the hodoscope. The forward plane has the 20 G10-card fingers and the remaining four short-base fingers. The tubes and bases for the forward plane extend to the high theta side of the hodoscope. The forward plane of the rear hodoscope package is $10 \mathrm{H} 04 \mathrm{Y}$, and the rear plane is $10 \mathrm{H} 05 \mathrm{Y}$. The fingers are numbered from the bottom. Plane 10H04Y then has fingers $10 \mathrm{H} 04 \mathrm{Y} 01$ through $10 \mathrm{H} 04 \mathrm{Y}^{24}$, and plane $10 \mathrm{H} 05 \mathrm{Y}$ has fingers $10 \mathrm{H} 05 \mathrm{Y} 01$ through $10 \mathrm{H} 05 \mathrm{Y} 25$.

\section{A.2.2 Assembly Notes}

The lowest 2 (next 2 are pretty high too) fingers in $10 \mathrm{H} 05 \mathrm{Y}$ require very high operating voltages, as do the fingers 1,2 , and 5 in $10 \mathrm{H} 04 \mathrm{Y}$. These fingers were chosen for these positions so that the high momentum resolution of the spectrometer would not be affected if these fingers could not handle long periods at the voltage needed to gain match them with the other fingers. Additionally, the lowest finger in $10 \mathrm{H} 05 \mathrm{Y}$ has a bent light-guide. When installed, the optical couplings between the finger and light-guide and between the light-guide and PMT are under stress. This finger was 
placed here so that the high momentum resolution would be unaffected if it were to fail.

The details of the gain matching and characterization of the hodoscope fingers in this plane are described in $\mathrm{N}$. Benmouna s note [149]. The internal alignment data for hodoscope planes $10 \mathrm{H} 04 \mathrm{Y}$ and $10 \mathrm{H} 05 \mathrm{Y}$, as well as for the lead passive preradiator (PbPPR), are given in P. DePietro's note [1.50].

\section{A.2.3 Rough Positions of the Rear Detector Package Components}

The preradiator and total absorber positions are those reported in Tech Note 5 (20 January, 1997 revision) [93]. Positions of the rear hodoscope planes and the lead passive preradiator are estimated from their positions relative to the shower calorimeter.

The location of the front of the preradiator stack was calculated to be at a $Z$ coordinate of $1740 \mathrm{~cm}$. The $Y$ coordinate of the top of the shower calorimeter deck plate was calculated to be at a $Y$ of $-154.9 \mathrm{~cm}$. The pre-survey locations of $10 \mathrm{H} 04 \mathrm{Y}$. $10 \mathrm{H} 05 \mathrm{Y}$, and the PbPPR (or "Pepper") are in reference to this coordinate of the deck plate at the front of the preradiator stack. The $Z$ coordinate of the hodoscope planes is that of the center of the finger. P. DePietro's note [1.50] contains the detailed measurements of the hodoscope and PbPPR used to calculate these data.

$\begin{array}{rcccc}\text { Component } & Z & \text { X Width } & \text { Y Bottom } & \text { Y Top } \\ \text { 10H04 } & 1734 \mathrm{~cm} & 37.9 \mathrm{~cm} & -146.5 \mathrm{~cm} & -83.1 \mathrm{~cm} \\ \text { 10H05 } & 1735 \mathrm{~cm} & 37.9 \mathrm{~cm} & -147.2 \mathrm{~cm} & -81.8 \mathrm{~cm} \\ \text { PbPPR } & 1738 \mathrm{~cm} & 38.5 \mathrm{~cm} & -147.2 \mathrm{~cm} & -76.8 \mathrm{~cm} \\ \text { PR-center } & 1743 \mathrm{~cm} & 37.5 \mathrm{~cm} & -146.1 \mathrm{~cm} & -83.7 \mathrm{~cm} \\ \text { TA-front } & 1747 \mathrm{~cm} & 44.1 \mathrm{~cm} & -154.9 \mathrm{~cm} & -81.3 \mathrm{~cm}\end{array}$

Table A.1: Estimated positions of the elements of the E $155 \times 10.5^{\circ}$ rear detector package. These positions assumed the PR $Z$ location was $1743.3 \mathrm{~cm}$, so they should all be moved back by $4 . \overline{c m}$ (see Section A.4). 


\section{A.2.4 Finger Assembly Diagrams}

The light-guides were produced as two components. the button and the trapezoid. The buttons are $0.325 "$ thick slices of $0.75 "$ diameter acrylic rod. The buttons have a rectangular slot $0.1625 \%$ deep and $0.325 \%$ wide cut in one face. The trapezoids are cut from $0.325^{\prime \prime}$ acrylic sheet. The lengths of the bases are $0.65{ }^{\prime \prime}$ and $1.0 "$. and the height of the trapezoid is $1.5 "$. The short base of the trapezoid is epoxied into the slot in the button.

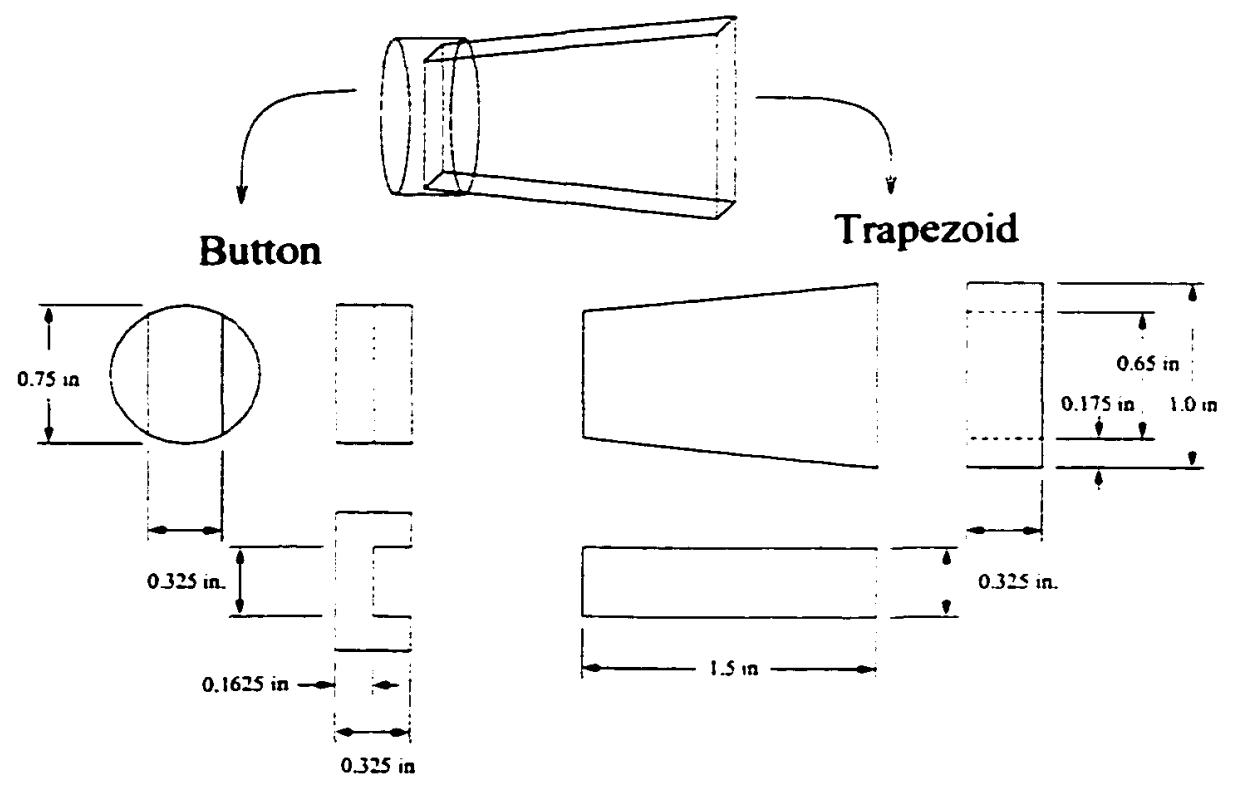

Figure A.1: E155X rear hodoscope lightguide diagrams 


\section{A.2.5 Photomultiplier Tube \& Base Assembly Diagrams}

Two types of photomultiplier assemblies were used in the construction of the 10.5 Frame 3 hodoscope. The dimensions of the tubes and bases are shown in Figure A.2.
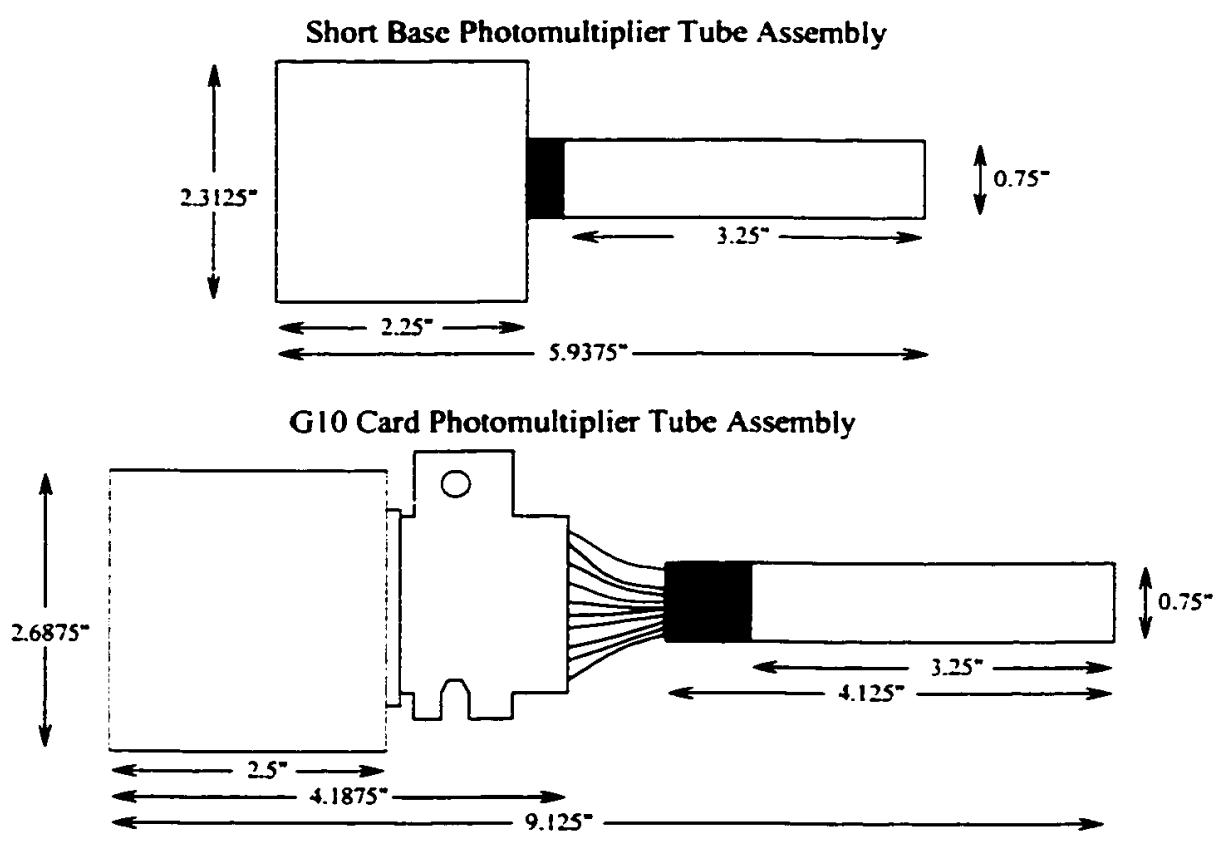

Figure A.2: E155X rear hodoscope photomultiplier tube assembly diagrams.

\section{A.3 Repairs to the Total Absorber Stack}

During the electronics checkout before E15.5X. TA block (3.4) was found to be unresponsive. It was believed to be due to the PMT dying. In order to replace the PMT, the backing plates from both block (3.4) and (3.5) had to be removed. In the process of doing this, it was found that the PMT housing of both blocks had broken off of the lead glass, and that the light-tight wrapping of block (3.4) had been compromised. This was the cause of the lack of signal in block (3.4). The housing of both blocks needed to be repaired, and the PMTs reinstalled without unstacking the total absorber.

The PMT housings of both blocks were removed and cleaned. The edges of the 
light-tight wrapping of blocks $(3.4)$ and $(3,5)$ were secured to the neighboring blocks using black electrical tape. The housings were reattached to the lead glass using 5 minute epoxy. The rear edges of the housings were covered with black silicone caulk to form a new light seal around the rear of the blocks. The blocks were visually inspected for light leaks before installing the tubes by shining a flashlight down the PMT port.

Due to concerns about possible damage to P.MT used for block (3.4). the PMT was replaced by a spare PMT. The tube and base were \#24 (using the early E1.5.5 numbering system), and were replaced by tube and base \#116 (in the $8 \mathrm{GeV}$ spectrometer pseudo-notation). The PMT used for block (3.5) was re-installed. In both cases, a plastic cookie was affixed to the PMT face using optical grease. then the cookie+PMT assembly was affixed to the lead glass with optical grease. The back of the PMTs were taped to the $\mu$-metal shield to form the rearward light seal. The support plates were reattached, to keep the PMT pressed against the lead glass. After the PMTs were reinstalled. final light checking was done. and the few additional leaks found were filled with the black caulk.

The initial high voltage setting used for block (3.4) in E155X was $1844 \mathrm{~V}$. This was determined by matching the LED signal peak in the block to the LED signal peak in that block during E155 $g_{2}$ running.

\section{A.4 Effects of the $10.5^{\circ}$ Calorimeter $Z$ "Shift"}

As was mentioned in Section 3.5.3, the shower calorimeter was believed to have a $Z$ position of the center of the PR of $Z=1743.3 \mathrm{~cm}$ for E15.5, but the surveys done for E15.5X indicated that the actual position of the center of the PR was $Z=1748.0 \mathrm{~cm}$. This represents a "shift" between the actual position and the position used in the E1.55 analysis of $4.7 \mathrm{~cm}$. This shift can affect the tracking analysis through the time-of-flight correction to the shower calorimeter times and 
through the slope of the trajectories used in momentum reconstruction.

\section{A.4.1 Effects on the time-of-flight correction}

The time-of-flight correction to the calorimeter time is given by $Z / c$. with a small angular correction (see Eq. 5.9). The use of $Z=1743.3 \mathrm{~cm}$ instead of the actual position of $Z=1748.0 \mathrm{~cm}$ produces a time-of-flight correction which is about $0.16 \mathrm{~ns}$ too small; this is within the timing resolutions used in the analysis (about 1 ns), but could cause a few tracks with marginal timing chi-squared to be discarded. or to have some subsystem events not included in the track (such as a Cherenkov event).

\section{A.4.2 Effects on the calculation of the trajectory slope}

The reconstruction of the particle trajectories uses the change in $Y$ and $Z$ between the hodoscope and the shower calorimeter to determine the slope of the trajectory, and thus the particle momentum. The rear-most hodoscope fingers in E15.5 had $Z=1225.1 \mathrm{~cm}$ (see Table 3.3 ), so the E15.5 value of $\Delta Z$ is $\Delta Z_{\mathrm{E} 155}=.518 .2$ $\mathrm{cm}$ and the $\Delta Z$ using the E15.5X $Z$ position is $\Delta Z_{\mathrm{E} 155 \mathrm{X}}=522.9 \mathrm{~cm}$. The central trajectory makes an angle with the horizontal of about $-100 \mathrm{mrad}$, so the $\Delta Y$ of the central trajectory to the E15.5X $Z$ position would be about $\Delta Y_{E_{155 X}}=-52.20$ $\mathrm{cm}$. Using this value of $\Delta Y$ with the E15.5 $Z$ position to calculate the reconstructed angle gives

$$
\theta_{\text {recon }}=\sin -1\left(\Delta Y_{E_{155 x}} / \Delta Z_{E_{155}}\right)=\sin -1(-0.100 \tau)=-0.1009
$$

The E15.5 $Z$ position results in a reconstructed angle within the spectrometer which is $0.9 \%$ higher than the actual angle.

Another way to examine the effect of the shift on the track reconstruction is to determine the $Y_{S H W}$ which would correspond to a particular slope. As was discussed 
in the preceding paragraph. the central trajectory, with an angle in the spectrometer of $-100 \mathrm{mrad}$. has $\Delta Y_{\mathrm{E}_{155 \mathrm{X}}}=-52.20 \mathrm{~cm}$ when the $E 1.5 .5 \mathrm{X} Z$ position is used. For the E1.55 $Z$ position, the central trajectory would have $\Delta Y_{E_{155}}=-.51 .73 \mathrm{~cm}$. Therefore the shift in $Z$ roughly corresponds to an error of $4.7 \mathrm{~mm}$ in the vertical position of the shower calorimeter. Recalling that the vertical position resolution of the shower calorimeter is given approximately by the width of the PR bars. $6.2 \mathrm{~cm}$. it wouic be expected that the shift would have little effect on most tracks.

A final comment about the track reconstruction is that the cursory verification of the $10.5^{\circ}$ momentum reconstruction from T418 data [114] also used the E1.5.5 $Z$ position information, and that the momentum reconstruction was accurate to within the uncertainties due to the granularity of the system. Therefore. to within the uncertainty, the $Z$ shift did not affect the momentum reconstruction for $\mathrm{T} 418$ or E15.5.

\section{A.4.3 Observed effect of the $Z$ shift in E15.5X}

Figure A.3 shows the effect of the $Z$ shift on $d Y / d Z$ and $E / P$ for a typical E15.5X run. Using the correct $Z$ (referred to as "new" in the figure). 22 additional electron tracks were identified; the total number of tracks remained about the same. at about twice the number of electron tracks. Thus the corrected positions give an electron rate that is about $0.5 \%(22 / 464.5)$ larger than the old positions. Recall that E15.5X had a substantially improved tracking system, so the small effects which are visible in the E1.5.5X data are washed out in the more granular E1.5.5 tracking system. 


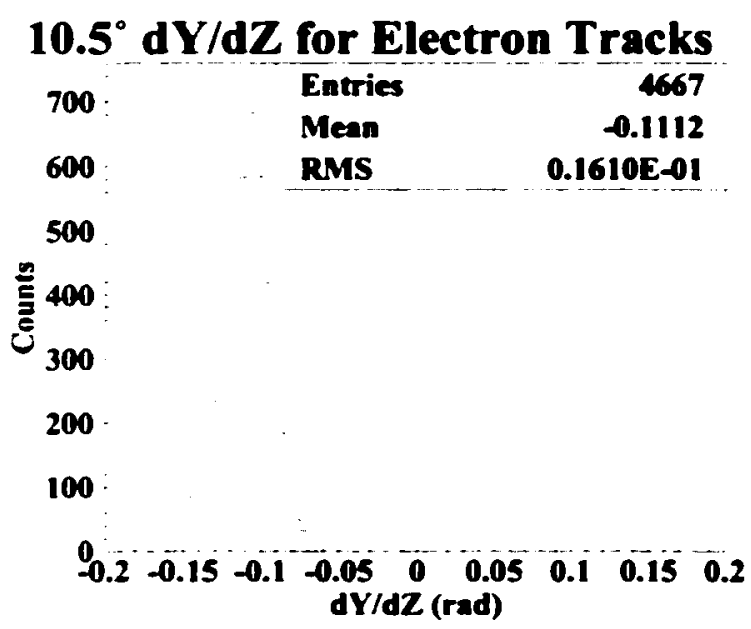

(a) $d Y / d Z$ with new $Z$

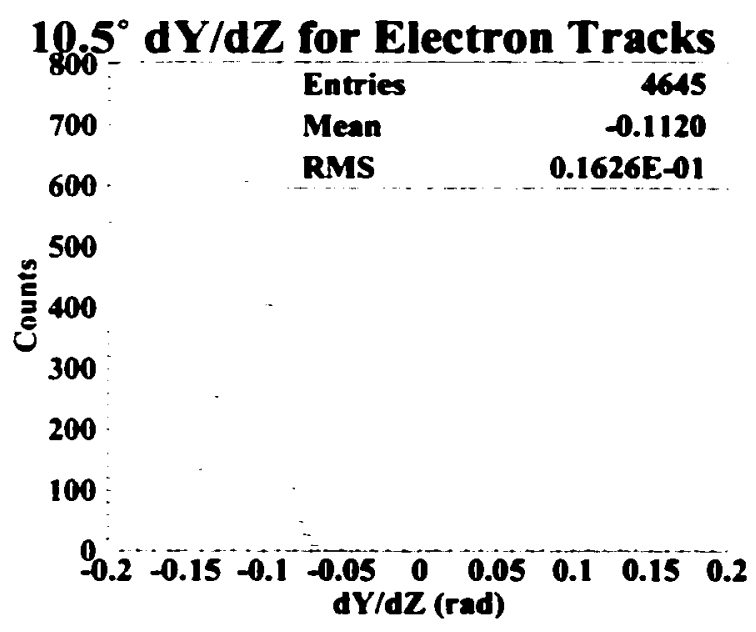

(c) $d Y / d Z$ with old $Z$

$\begin{array}{ccr}10.5^{\circ} \mathrm{E} / \mathrm{P} \text { for Electron Tracks } \\ \mathbf{5 0 0} & \begin{array}{c}\text { Entries } \\ \text { Mean }\end{array} & \mathbf{6 6 6 7} \\ & \text { RMS } & 0.9758 \\ 400 & & 0.1034\end{array}$

$\overbrace{200}^{50}$

100

$0_{0} 0.20 .40 .6 \quad 0.8 \underset{E / P}{1} 1.21 .4 \begin{array}{lllllll}1.6 & 1.8 & 2\end{array}$

(b) $E / P$ with new $Z$

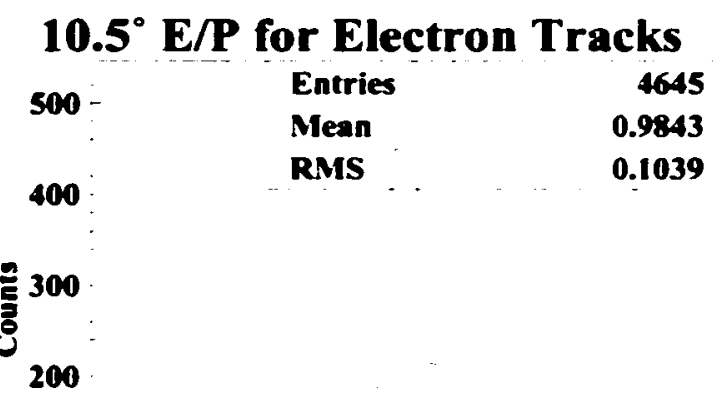

100

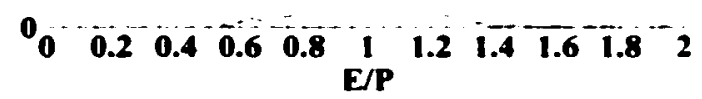

(d) $E / P$ with old $Z$

Figure A.3: Plots of trajectory slope, $d Y / d Z$, and $E / P$ for electron tracks in a typical E155X run, where the old and new $Z$ positions have been used in the analysis. 


\section{APPENDIX B}

\section{Bjorken $x$ in the infinite momentum frame}

In the reference frame where the proton's momentum is infinite (the infinite momentum frame), the transverse momentum of the partons within the proton may be neglected. Taking one parton to have 3-momentum $x P$ and effective mass $a . M$. the remaining partons have a total 3 -momentum $(1-x) P$ and total effective mass $(1-a) M$.

$$
\begin{aligned}
& E^{2}=P^{2}+M^{2} \\
& E_{\mathrm{l}}^{2}=(x P)^{2}+(a M)^{2} \\
& E_{r}^{2}=(1-x)^{2} P^{2}+(1-a)^{2} M^{2}
\end{aligned}
$$

Since the total energy, $E$. is the sum of $E_{1}+E_{\mathrm{r}}$. the square of the energy is:

$$
\begin{aligned}
P^{2}+M^{2}= & \left(E_{1}+E_{r}\right)^{2} \\
= & E_{1}^{2}+E_{r}^{2}+2 E_{1} E_{r} \\
= & \left(P^{2}+M^{2}\right)+2\left((x P)^{2}+(a M)^{2}\right)-2\left(x P^{2}+a \cdot M^{2}\right) \\
& +2 E_{1} E_{r} \\
= & \left(P^{2}+M^{2}\right)+2\left(E_{1}^{2}+E_{1} E_{r}\right)-2\left(x P^{2}+a M^{2}\right) \\
= & \left(P^{2}+M^{2}\right)+2 E_{1} E-2\left(x P^{2}+a M^{2}\right) .
\end{aligned}
$$

Which simplifies to

$$
E_{1} E=\left(x P^{2}+a M^{2}\right)
$$

Squaring Eq. B.10, and substituting Eq. B.1 and Eq. B.2 for $E$ and $E_{1}$.

$$
x^{2} P^{4}+x^{2} M^{2} P^{2}+a M^{2} P^{2}+a^{2} M^{4}=\left(x P^{2}+a M^{2}\right)^{2}
$$




$$
x^{2} P^{4}+x^{2} M^{2} P^{2}+a M^{2} P^{2}+a^{2} M^{4}=x^{2} P^{4}+2 a x M^{2} P^{2}+a^{2} M^{4} .
$$

Then. collecting terms,

$$
\begin{aligned}
\left(x^{2}-2 a x+a^{2}\right) M^{2} P^{2} & =0 \\
(x-a)^{2} M^{2} P^{2} & =0 .
\end{aligned}
$$

The momentum fraction $x$ and the mass fraction $a$ are identical. and we now identify them as $x$.

Therefore, the parton with momentum fraction $x$ has an effective mass $x: M$. So the 4-momentum of the parton is $x P^{\mu}$. where $P^{\mu}$ is the total 4-momentum of the nucleon. 


\section{BIBLIOGRAPHY}

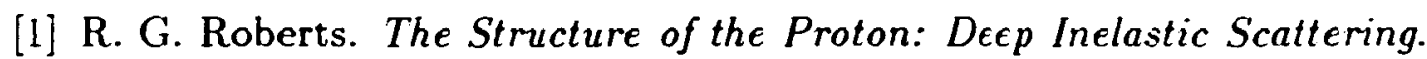
Cambridge University Press. New York, NY, 1990.

[2] B. Lampe and E. Reya. Spin physics and polarized structure functions. Eprint hep-ph/9810270, 1998.

[3] L. W. Whitlow. Deep Inelastic Structure Functions From Electron Scattering on Hydrogen, Deuterium, and Iron at $0.6 \mathrm{GeV}^{2} \leq Q^{2} \leq 30.0 \mathrm{GeV}^{-2}$. PhD thesis, Stanford Linear Accelerator Center, Stanford University. Stanford. CA, 1990. Also SLAC-Report-357.

[4] Particle Data Group. Review of particle physics. Eur. Phys. J. C. 3:1- 794 . 1998.

[5] L. W. Whitlow et al. Precise measurements of the proton and deuteron structure functions from a global analysis of the SLAC deep inelastic electron scattering cross sections. Phys. Lett., B282:475-482. 1992.

[6] S. Aid et al. A measurement and QCD analysis of the proton structure function $F_{2}\left(x, Q^{2}\right)$ at HERA. Nucl. Phys., B4i0:3-38. 1996.

[7] C. Adloff et al. A measurement of the proton structure function $F_{2}\left(x, Q^{2}\right)$ at low $x$ and low $Q^{2}$ at HERA. Nucl. Phys., B497:3-28. 1997.

[8] M. Derrick et al. Measurement of the $F_{2}$ structure function in deep inelastic $e^{+} p$ scattering using 1994 data from the ZEUS detector at HERA. Z. Phys.. C.72:399-424, 1996.

[9] J. Breitweg et al. Measurement of the proton structure function $F_{2}$ and ${\sigma_{\text {tot }}^{\circ}}^{r^{*} p}$ at low $Q^{2}$ and very low $x$ at HERA. Phys. Lett.. B407:432-448. 1997.

[10] M. Arneodo et al. Measurement of the proton and the deuteron structure functions, $F_{2}^{p}$ and $F_{2}^{d}$. Phys. Lett., B364:10i-115. 1995. 
[11] M. R. Adams et al. Proton and deuteron structure functions in muon scattering at $470 \mathrm{GeV}$. Phys. Rev.. D54:3006-3056, 1996.

[12] A. C. Benvenuti et al. A high statistics measurement of the proton structure functions $F_{2}\left(x, Q^{2}\right)$ and $R$ from deep inelastic muon scattering at high $Q^{2}$. Phys. Lett., B223:485-489. 1989.

[13] A. C. Benvenuti et al. A high statistics measurement of the deuteron structure functions $F_{2}\left(x, Q^{2}\right)$ and $R$ from deep inelastic muon scattering at high $Q^{2}$. Phys. Lett., B237:592-598. 1990.

[14] K. Abe et al. Measurements of $R=\sigma_{L} / \sigma_{T}$ for $0.03<x<0.1$ and fit to world data. Phys. Lett.. B452:194-200. 1999.

[15] L. W. Whitlow et al. A precise extraction of $R=\sigma_{L} / \sigma_{T}$ from a global analysis of the SLAC deep inelastic e-p and e-d scattering cross sections. Phys. Lett., B250:193-198. 1990.

[16] L. H. Tao et al. Precision measurement of $R=\sigma_{L} / \sigma_{T}$ on hydrogen. deuterium and beryllium targets in deep inelastic electron scattering. $Z$. Phys.. C70:387-390, 1996.

[17] M. Arneodo et al. Measurement of the proton and deuteron structure functions, $F_{2}^{p}$ and $F_{2}^{d}$, and of the ratio $\sigma_{L} / \sigma_{T}$. Nucl. Phys., B483:3-43. 1997.

[18] P. Berge et al. A measurement of differential cross-sections and nucleon structure functions in charged-current neutrino interactions on iron. $Z$. Phys., C49:187-223, 1991.

[19] Yu. L. Dokshitzer. Calculation of the structure functions for deep inelastic scattering and e+ e- annihilation by perturbation theory in quantum chromodynamics. (in Russian). Sov. Phys. JETP, 46:641-65.3, 197 i

[20] V. N. Gribov and L. N. Lipatov. Deep inelastic e p scattering in perturbation theory. Yad. Fiz., 15:781-807, 1972. Sov. J. Nucl. Phys. 15:438-450.1972.

[21] V. N. Gribov and L. N. Lipatov. et e- pair annihilation and deep inelastic e p scattering in perturbation theory. Yad. Fiz., 15:1218-1237. 1972. Sov. J. Nucl. Phys. 15:675-684,1972.

[22] G. Altarelli and G. Parisi. Asymptotic freedom in parton language. Nucl. Phys., B126:298-318, 1977. 
[23] Yu. G. Kolomensky. Precision Measurement of the Neutron Spin Dependent Structure Functions. PhD thesis, University of Massachusetts, Amherst. 1997 .

[24] M. Gluck, E. Reya, and A. Vogt. Dynamical parton distributions revisited. Eur. Phys. J., C5:461-470, 1998.

[2.5] R. Mertig and W. L. van Neerven. The calculation of the two-loop spin splitting functions $P_{i j}^{(1)}(x)$. Z. Phys., C70:637-6.53. 1996.

[26] W. Vogelsang. Rederivation of the spin-dependent next-to-leading order splitting functions. Phys. Rev., D54:2023-2029. 1996.

[27] P. Bosted. Polarized asymmetry formulas. E143 Technical Note 19. Stanford Linear Accelerator Center. August 1993. Unpublished.

[28] $\mathrm{K}$. Abe et al. Measurements of the proton and deuteron spin structure functions $g_{1}$ and $g_{2}$. Phys. Rev., D58:112003, 1998.

[29] M. J. Alguard $\epsilon t$ al. Deep-inelastic e-p asymmetry measurements and comparison with the Bjorken sum rule and models of proton spin structure. Phys. Rev. Lett., 41:70-73, 1978.

[30] G. Baum et al. New measurement of deep-inelastic e-p asymmetries. Phys. Rev. Lett.. 51:1135-1138, 1983.

[31] P. L. Anthony et al. Deep inelastic scattering of polarized electrons by polarized ${ }^{3} \mathrm{He}$ and the study of the neutron spin structure. Phys. Rev.. D.54:6620-66.50. 1996.

[32] K. Abe $\epsilon t$ al. Precision determination of the neutron spin structure function $g_{1}^{n}$. Phys. Rev. Lett., i9:26-30. 1997.

[33] $\mathrm{K}$. Abe $\epsilon t$ al. Measurement of the neutron spin structure function $g_{2}^{n}$ and asymmetry $A_{2}^{n}$. Phys. Lett., B404:377-382, 1997.

[34] J. Ashman et al. A measurement of the spin asymmetry and determination of the structure function $g_{1}$ in deep inelastic muon-proton scattering. Phys. Lett., B206:364-370, 1988.

[35] J. Ashman et al. An investigation of the spin structure of the proton in deep inelastic scattering of polarized muons on polarized protons. Nucl. Phys., B328:1-3.5, 1989. 
[36] B. Adeva et al. Spin asymmetries $A_{1}$ and structure functions $g_{1}$ of the proton and the deuteron from polarized high energy muon scattering. Phys. Rev.. D58:112001, 1998.

[3i] B. Adeva et al. Spin asymmetries $A_{1}$ of the proton and the deuteron in the low $x$ and low $Q^{2}$ region from polarized high energy muon scattering. Phys. Rev., D60:072004, 1999.

[38] $\mathrm{K}$. Ackerstaff et al. Measurement of the neutron spin structure function $g_{1}^{n}$ with a polarized ${ }^{3} \mathrm{He}$ internal target. Phys. Lett.. B404:383-389, 1997.

[39] A. Airapetian et al. Measurement of the proton spin structure function $g_{1}^{p}$ with a pure hydrogen target. Phys. Lett.. B442:484-492. 1998.

[40] R. L. Jaffe and Aneesh Manohar. The $g_{1}$ problem: Fact and fantasy on the spin of the proton. Nucl. Phys., B337:509-546. 1990.

[41] $\mathrm{h}$. Abe et al. Next-to-leading order QCD analysis of polarized deep inelastic scattering data. Phys. Lett., B405:180-190. 1997.

[42] P. L. Anthony et al. Measurement of the proton and deuteron spin structure functions $g_{2}$ and asymmetry $A_{2}$. Phys. Lett.. B458:529-5.35. 1999.

[4.3] M. E. Peskin and D. V. Schroeder. An Introduction to Quantum Field Theory. Addison-Wesley, Reading, MA. 1995.

[44] S. Wandzura and F. Wilczek. Sum rules for spin-dependent electroproduction- test of relativistic constituent quarks. Phys. Lett.. B $72: 195-198,1977$.

[45] J. D. Bjorken. Applications of the chiral $U(6) \odot U(6)$ algebra of current densities. Phys. Rev., 148:1467-1478, 1966.

[46] S. A. Larin and J. A. M. Vermaseren. The $\alpha_{S}^{3}$ corrections to the Bjorken sum rule for polarized electroproduction and to the Gross-Llewellyn Smith sum rule. Phys. Lett., B259:345-352, 1991.

[47] G. S. Mitchell. A Precision Measurement Of The Spin Structure Function $g_{1}\left(x, Q^{2}\right)$ For The Proton and Deuteron. PhD thesis. University of Wisconsin - Madison, Madison, Wisconsin, 1999.

[48] H. Burkhardt and W. N. Cottingham. Sum rules for forward virtual Compton scattering. Annals Phys., 56:453-463. 1970. 
[49] A. V. Efremov. O. V. Teryaev. and E. Leader. Exact sum rule for transversely polarized DIS. Phys. Rev.. D55:430 T-4314. 1997.

[50] J. E. Clendenin et al. The SLAC polarized electron source. SLAC-PUB-7619. Talk given at 7 th International Workshop on Polarized Gas Targets and Polarized Beams. Urbana. IL. 18-22 Aug 1997.

[51] D. Schultz et al. The high peak current polarized electron source of the Stanford Linear Collider. Nucl. Instr. and Meth., A340:127-132. 1994.

[52] R. Alley et al. The Stanford linear accelerator polarized electron source. Nucl. Instr. and Meth., A365:1-27, 199.5.

[53] R. B. Neal, editor. The Stanford Two Mile Accelerator. W. A. Benjamin. Inc., New York, NY. 1968.

[54] J. M. Bauer. Measurement of the Longitudinal Deuteron Spin-Structure Function in Deep Inelastic Scattering. PhD thesis. University of Massachusetts, Amherst, 1996.

[5.5] Z. D. Farkas, H. A. Hoag. G. A. Loew, and P. B. Wilson. SLED: A method of doubling SLAC's energy. SLAC-PLB-1453. Presented at 9th Int. Conf. on High Energy Accelerators, SLAC, Stanford. Calif.. May 2-i. 1974.

[56] F. J. Decker. Z. D. Farkas, and J. Turner. High current long beam pulse with SLED. SLAC-PUB-8113. Contributed to IEEE Particle Accelerator Conference (PAC 99), New York, NY. 29 Mar - 2 Apr 1999.

[5i] R. Erickson et al. First operation of the upgraded SLAC A-line. SLAC-PUB-7177.

[58] L. Stuart. E154 beam energies and spin precession. E154 Technical Note 6. Stanford Linear Accelerator Center, June 199.5. Lnpublished.

[59] S. Kuhn and M. Hing. Toroid calibration for E143. E143 Technical Note 88. Stanford Linear Accelerator Center, January 1995. Unpublished.

[60] L. Sorrell. Private Communication.

[61] H. R. Band et al. A Møller polarimeter for high energy electron beams. Nucl. Instr. and Meth., A400:24-33, 1997.

[62] J. Arrington et al. A variable energy Møller polarimeter at the MIT-Bates Linear Accelerator Center. Nucl. Instr. and Meth., A311:39-48. 1992. 
[6.3] M. Swartz et al. Observation of target electron momentum effects in single-arm Møller polarimetry. Nucl. Instr. and .Meth.. A36.3:526-537. 1995.

[64] L. G. Levchuk. The intra-atomic motion of bound electrons as a possible source of a systematic error in electron beam polarization measurements by means of a Møller polarimeter. Nucl. Instr. and Meth.. A345:496-499. 1994.

[65] H. R. Band, G. Mitchell. R. Prepost. and T. Wright. E-154 Møller polarimeter. E154 Technical Note 40. Stanford Linear Accelerator Center. August 1996. Unpublished.

[66] H. Band. Beam polarization status. E15.5 Collaboration Meeting. February 1998. Enpublished.

[67] P. Steiner et al. A high-rate coincidence Møller polarimeter. Nucl. Instr. and . Weth.. A419:105-120, 1998.

[68] T. Wright. Møller polarimetry status. E1.5.5 Collaboration Meeting. December 1996. Unpublished.

[69] H. Band (reported by J. Johnson). E1.5.5 beam polarization status. E1.5.5 Collaboration Meeting, June 1998. Unpublished.

[70] D. G. Crabb and D. B. Day. The Virginia/Basel/SL.AC polarized target: operation and performance during experiment E143 at SLAC. Nucl. Instr. and Meth.. A356:9-19, 1995. Also E143 Technical Note $i t$.

[11] D. G. Crabb and W. Meyer. Solid polarized targets for nuclear and particle physics experiments. Annu. Rev. Nucl. Part. Sci, 47:6i-109. 1997. Also E155 Technical Note 50 .

[־2] A. Tobias. E155 target model. E15.5 Technical Note 36. Stanford Linear Accelerator Center, July 1997. Unpublished.

[i3] S. Bültmann $e t$ al. A study of lithium deuteride as a material for a polarized target. Nucl. Instr. and Meth., A425:23-36. 1999. Also E1.55 Technical Note 67 .

[74] P. Bosted. Solid targets for E15.5. E15.5 Technical Note 11. Stanford Linear Accelerator Center, July 1996. Unpublished.

[75] T. D. Averett. Measurement Of The Spin Structure Function $g_{2}\left(x, Q^{2}\right)$ For The Proton and Deuteron. PhD thesis, University of Virginia, 1995. 
[i6] G. R. Court et al. A high precision Q-meter for the measurement of proton polarization in polarized targets. Nucl. Instr. and Meth.. A324:433-440. 1993.

[i]] D. E. Reyna. Measurement Of The Spin Structure Function $g_{2}\left(x \cdot Q^{2}\right)$ For The Neutron. PhD thesis, The American Lniversity. Washington. D.C. $20016,1998$.

[78] E1.5.5 Collaboration. Proposal to add a spectrometer to E15.5. E1.5.5 Technical Note 8, Stanford Linear Accelerator Center. February 1996. Unpublished.

[79] P. Bosted. The 10.5 degree spectroneter (Revised 2 October 1996). E1.5.5 Technical Note 10, Stanford Linear Accelerator Center. October 1996. Unpublished.

[s0] G. Petratos. R. L. Eisele, R. Gearhart. Emlyn Hughes, and C. Young. Large acceptance magnetic spectrometers for polarized deep inelastic electron scattering. SLAC-PUB-5678. Presented at the IEEE Nuclear Science Symp. and Medical Imaging Conf., Santa Fe, NM. Nov 5-9. 1991.

[81] W.R. Leo. Techniques for Nuclear and Particle Physics Experiments. Springer-Verlag. New York. NY, 2nd edition, 1994.

[82] Y. Tomkiewicz and E. L. Garwin. Optical absorption spectra of some potentially interesting gases for Cherenkov counters. Vucl. Instr. and Weth. $114: 413-416.1974$.

[83] C. Y. Prescott. Cerenkov counter threshold pressures for E15.5 gas mixtures. E15.5 Technical Note 25. Stanford Linear Accelerator Center. December 1996. Unpublished.

[84] E. L. Garwin, Y. Tomkiewicz, and D. Trines. Method for elimination of quartz-face phototubes in Cherenkov counters by use of wavelength-shifter. Nucl. Instr. and Meth., 107:365-370. 1973.

[85] M. Buénerd. Vacuum tightness requirements for the E15.5 cerenkov counters from their contamination by oxygen and water vapor in E1.54. E1.5.5 Technical Note 22, Stanford Linear Accelerator Center, October 1996. Unpublished.

[86] L. Sorrell. Test information on mirrors used for e15.5/e15.5x cherenkov system. E155 Technical Note 82. Stanford Linear Accelerator Center. April 1999. Unpublished. 
[S7] L. Sorrell. Information on PMTs used for E154/E15.5/E15.5x Cherenkov system. E155 Technical Note 81, Stanford Linear Accelerator Center. June 1999. Unpublished.

[88] R. Erbacher. SLAC. E143 Čerenkov technical report. E143 Technical Note 87. Stanford Linear Accelerator Center. January 199.5. Unpublished.

[89] G. T. Bartha et al. Design and performance of the ASP lead-glass calorimeter. Nucl. Instr. and Meth.. A275:59-70, 1989.

[90] S. Incerti and F. Sabatié. E155 updates of the E154 clustering algorithm. E15.5 Technical Note 31, Stanford Linear Accelerator Center. April 1997. Unpublished.

[91] P. L. Anthony and Z. M. Szalata. Flexible high performance VME based data acquisition system for the ESA physics program. SLAC-PLB-7201.

[92] P. N. Kirk et al. Elastic electron-proton scattering at large four-momentum transfer. Phys. Rev., D8:63-91. 1973.

[93] P. Bosted. 10.5 degree spectrometer parameters. E15.5 Technical Note 5. Stanford Linear Accelerator Center. January 1997. Inpublished.

[94] S. Kadlec and R. Liridgren. Design and construction of the 10 degree spectrometer scintillator hodoscope for E1.5.5 at SLAC. E1.5.5 Technical Note 20. Stanford Linear Accelerator Center, September 1996. Unpublished.

[95] AMI Metals/Kaiser Aluminium. Report Serial Number 0132-0498.

[96] T. Toole. cergeom10.r0; E155 production code, 15 December 1997 version. Unpublished, December 1997.

[97] L. Sorrell. Summary of Cerenkov hardware information. E15.5 Technical Note 49. Stanford Linear Accelerator Center. February 1998. Unpublished.

[98] T. Toole. E15.5 Cherenkov detector performance. E1.5.5 Technical Note 99. Stanford Linear Accelerator Center, August 1999. Unpublished.

[99] M. C. Berisso. Modifications to the shower counter electronics for E1.5.5. E1.5.5 electronics diagram for 10.5 degree spectrometer (updated after E1.5.5). E15.5 Technical Note 1, Update, Stanford Linear Accelerator Center. 1997. Unpublished. 
[100] G. G. Petratos. Quasielastic Electron-Deuleron Scattering at $180^{\circ}$ at Large Momentum Transfers. PhD thesis. The American Lniversity. 1988.

[101] A. Lung. Quasielastic Electron-Deuteron Scattering and Veutron Electromagnetic Form Factors at $Q^{2}=1.7 .5$ to $4.0(\mathrm{GeV} / \mathrm{c})^{2}$. PhD thesis. The American University, 1992.

[102] S. R. Dasu. Precision Measurement of $x . Q^{2}$ and A-Dependence of $R=\sigma_{L} / \sigma_{T}$ and $F_{2}$ in Deep Inelastic Scattering. $\mathrm{PhD}$ thesis. University of Rochester, 1988.

[10:3] R. C. D. Walker. A Measurement of the Proton Elastic Form Factors for $1<Q^{2}<3(\mathrm{GeV} / \mathrm{c})^{2}$. PhD thesis, California Institute of Technology: 1989.

[104] L. Clogher. A Precise Measurement of the Proton Elastic Form Factors for $1.7<Q^{2}<8.83(\mathrm{GeV} / \mathrm{c})^{2}$. PhD thesis, American University. 199:3.

[105] N. P. Bansal and R. H. Doremus. Handbook of Glass Properties. Harcourt Brace Jovanovich, 1986.

[106] H. Rawson. Properties and Applications of Glass. Elsevier. 1980.

[10i] J. S. Beale et al. A lead-glass Cherenkov detector for electrons and photons. Nucl. Instr. and Weth., 117:501-508, 1974.

[108] L. Antoniazzi et al. The Experiment 705 electromagnetic shower calorimeter. Nucl. Instr. and Weth., A332:57-77. 1993.

[109] H. Borel et al. Shower counters for SLAC experiments E142/E143. IEEE Transactions on Nuclear Science. 42(4), August 199.5. Also E143 Technical Note 8.3.

[110] M. Bouadani. Cerenkov LED project summary. E1.55 Technical Note 61. Stanford Linear Accelerator Center. Summer 1996. Unpublished.

[111] E15.5 Collaboration. E155 logbooks and run sheets. 1997. Unpublished.

[112] P. Mchee. A correction to the proton polarization from radiation damage. E15.5 Technical Note 112. Stanford Linear Accelerator Center, May 2000. Unpublished.

[113] M. Cavalli-Sforza et al. A method of obtaining parasitic $e^{+}$or $e^{-}$beams during SLAC Linear Collider operation. IEEE Trans. Nucl. Sci.. 4l:1371-1373, 1994. Also SLAC-PUB-638i. 
[114] L. Sorrell. A cursory verification of the 10.5 degree momentum reconstruction. E155 Technical Note 56. Stanford Linear Accelerator Center. May 1998. Unpublished.

[115] A. Romano. Determination of voltage offsets for ADC's of total absorbers in 10.5 degree shower counter. E155 Technical Note 54. Stanford Linear Accelerator Center, April 1998. Linpublished.

[116] L. Sorrell. Magnetic field dependence of 10.5 degree shower counter TA gains for T418. E1.55 Technical Note 5.5. Stanford Linear Accelerator Center. May 1998. Unpublished.

[11i] C. Young. Polarization bits-from CID to analysis. E143 Technical Note 96. Stanford Linear Accelerator Center. May 1995. Unpublished.

[118] V. Breton et al. Application of neural networks and cellular automata to interpretation of calorimeter data. Nucl. Instr. and Meth.. A362:478-486. 1995.

[119] M. Gale and P. King. Slewing corrections for the $10.5^{\circ}$ shower counter. E1.5.5 Technical Note 40. Stanford Linear Accelerator Center. September 1997. Unpublished.

[120] P. Bosted. 10.5 degree electron definitions. E15.5 Technical Note 68. Stanford Linear Accelerator Center, September 1998. Linpublished.

[121] T. Toole. A ground level comparsion of two implementations of the $10.5 \mathrm{deg}$ hybrid definition. E15.5 Technical Note 76. Stanford Linear Accelerator Center, January 1999. Unpublished.

[122] P. Bosted. Target polarization study using physics asymmetries. E15.5 Technical Note 58, Stanford Linear Accelerator Center. May 1998. Unpublished.

[12:3] P. Mchee. Description of the interim proton polarization correction. E155 Technical Note 65, Stanford Linear Accelerator Center. July 1998. Unpublished.

[124] P. Bosted. Comparison of E155 and E15.5X polarization vrs. dose. E15.5 Technical Note 110, Stanford Linear Accelerator Center. May 2000. Unpublished.

[125] T. Toole. Data tests and checks. E155 Collaboration Meeting. February 1998. Linpublished. 
[126] T. Averett. Dilution factor and corrections for nitrogen and lithium polarizations. E15.5 Technical Note 27, Stanford Linear Accelerator Center. March 1997. Unpublished.

[127] O. Rondon. Dilution factor calculation. E15.5 Technical Note 14. Stanford Linear Accelerator Center, August 1996. Unpublished.

[128] A. Tobias. Lithium deuteride analysis. E1.5.5 Technical Note 78. Stanford Linear Accelerator Center, February 1999. Unpublished.

[129] J. D. Walecka. Theoretical nuclear and subnuclear physics. Oxford University Press. New York. NY. 1995.

[130] A. Tobias. Radiated dilution factor and packing fraction analysis. E1.5.5 Collaboration Meeting, June 1998. Unpublished.

[131] P. Bosted. E1.5.5 luminosity correction to the spectrometer rates. E1.5.5 Technical Note 63, Stanford Linear Accelerator Center, June 1998. Unpublished.

[132] P. E. Bosted. Electroweak correction to E154. E154 Technical Note 29. Stanford Linear Accelerator Center, January 199.5. Unpublished.

[133] P. Bosted. Resolution smearing. E155 Collaboration Meeting. June 1998. Unpublished.

[134] F. R. Wesselmann and S. E. Kuhn. Calculation of radiative corrections. E15.5 Technical Note 100. Stanford Linear Accelerator Center. September 1999. Unpublished.

[135] F. R. Wesselmann. Precision . Measurement Of The Spin Structure Of The Proton and Deuteron. PhD thesis. Old Dominion University. 1999.

[136] P. L. Anthony et al. Measurements of the $Q^{2}$-dependence of the proton and neutron spin structure functions $g_{1}^{p}$ and $g_{1}^{n}$. hep-ph/0007248. 2000 . Submitted to Phys. Lett. B.

[137] M. Stratmann. Bag model predictions for polarized structure functions and their $Q^{2}$-evolutions. Z. Phys., C60:763-7i1, 1993.

[138] X. Song. Polarized structure function $g_{2}$ in the c.m. bag model. Phys. Rec.. D54:1955-1966, 1996. 
[139] F. R. Wesselmann. GUF fit to global $g_{1} / F_{1}$ data set. E15.5 Technical Note 113. Stanford Linear Accelerator Center, July 2000. Linpublished.

[140] B. Adeva et al. A next-to-leading order qcd analysis of the spin structure function $g_{1}$. Phys. Rev.. D58:112002. 1998.

[141] X. Ji and P. Unrau. $Q^{2}$-dependence of the nucleon's $G_{1}$ structure function sum rule. Phys. Lett., B333:228-232, 1994.

[142] E. Stein, P. Gornicki, L. Mankiewicz, A. Schafer, and W. Greiner. QCD sum rule calculation of twist-3 contributions to polarized nucleon st ructure functions. Phys. Lett., B343:369-3i6, 1995.

[143] I. I. Balitsky, V. M. Braun, and A. V. Kolesnichenko. Power corrections $1 / Q^{2}$ to parton sum rules for deep inelastic scattering from polarized nucleons. Phys. Lett.. B242:245-250, 1990. Erratum-ibid. B318: 648. 199:3.

[144] B. Ehrnsperger and A. Schafer. Model for the $Q^{2}$ dependence of polarized structure functions. Phys. Rev.. D52:2709-2716. 1995.

[145] M. Gockeler $\epsilon t$ al. Polarized and unpolarized nucleon structure functions from lattice QCD. Phys. Rev.. D53:2317-2:325. 1996.

[146] H. Weigel. L. Gamberg, and H. Reinhardt. Polarized nucleon structure functions within a chiral soliton model. Phys. Rev.. D.55:6910-6923. 1997.

[147] M. Gockeler $\epsilon t$ al. The polarized structure function $g_{2}$ : A lattice study revisited. hep-ph/9909253. 1999.

[148] G. Mitchell. E155 NLO fit results. E15.5 Technical Note 101. Stanford Linear Accelerator Center, September 1999. Unpublished.

[149] N. Benmouna. Characterization of the fingers for the frame III hodoscopes. E15.5 Technical Note 72, Stanford Linear Accelerator Center. December 1998. Unpublished.

[150] P. DePietro. Internal alignment of the frame III hodoscope for the 10.5 degree spectrometer. E155 Technical Note 73. Stanford Linear Accelerator Center, December 1998. Unpublished. 


\section{VITA}

\section{Paul McConihay King II}

Paul McConihay King II was born in C'harleston. West Virginia. on 30 September 1972. Graduated from Burnt Hills-Ballston Lake High School in Burnt Hills. New York, in June 1990. Received a Bachelor of Arts degree in physics from the State University of New York College at Geneseo in Geneseo. New York in May 1994. Entered the physics graduate program at the College of William and Mary in Virginia in 1994. Received a Master of Science degree in physics from the College of William and Mary in Virginia in May 1996. This dissertation was defended on 14 July 2000 at the College of William and Mary in Virginia. 